\title{
Myeloproliferative neoplasia : more insight in the pathogenesis
}

Citation for published version (APA):

Koopmans, S. M. (2014). Myeloproliferative neoplasia : more insight in the pathogenesis. [Doctoral Thesis, Maastricht University]. Uitgeverij BOXPress. https://doi.org/10.26481/dis.20140424sk

Document status and date:

Published: 01/01/2014

DOI:

10.26481/dis.20140424sk

Document Version:

Publisher's PDF, also known as Version of record

\section{Please check the document version of this publication:}

- A submitted manuscript is the version of the article upon submission and before peer-review. There can be important differences between the submitted version and the official published version of record. People interested in the research are advised to contact the author for the final version of the publication, or visit the DOI to the publisher's website.

- The final author version and the galley proof are versions of the publication after peer review.

- The final published version features the final layout of the paper including the volume, issue and page numbers.

Link to publication

\footnotetext{
General rights rights.

- You may freely distribute the URL identifying the publication in the public portal. please follow below link for the End User Agreement:

www.umlib.nl/taverne-license

Take down policy

If you believe that this document breaches copyright please contact us at:

repository@maastrichtuniversity.nl

providing details and we will investigate your claim.
}

Copyright and moral rights for the publications made accessible in the public portal are retained by the authors and/or other copyright owners and it is a condition of accessing publications that users recognise and abide by the legal requirements associated with these

- Users may download and print one copy of any publication from the public portal for the purpose of private study or research.

- You may not further distribute the material or use it for any profit-making activity or commercial gain

If the publication is distributed under the terms of Article $25 \mathrm{fa}$ of the Dutch Copyright Act, indicated by the "Taverne" license above, 


\section{MYELOPROLIFERATIVE NEOPLASIA: MORE INSIGHT IN THE PATHOGENESIS}

Suzanne Marjolein Koopmans 
The printing of this thesis was generously supported by:

MPN stichting, patiëntenvereniging

Novartis, Pharmaceuticals

Author:

Suzanne M. Koopmans

Cover photo:

Dennis van Sint Fiet (dennisvansintfiet.nl)

Layout:

Ivo Van den Broek

Published by:

Uitgeverij BOXPress, 's Hertogenbosch

Printed by:

Proefschriftmaken.nl II Uitgeverij BOXPress

ISBN:

$978-90-8891-845-2$ 


\title{
MYELOPROLIFERATIVE NEOPLASIA: MORE INSIGHT IN THE PATHOGENESIS
}

\author{
PROEFSCHRIFT
}

ter verkrijging van de graad van doctor aan de Universiteit Maastricht op gezag van de Rector Magnificus, Prof dr. L.L.G. Soete volgens het besluit van het College van Decanen,

in het openbaar te verdedigen op

donderdag 24 april 2014 om 12.00 uur

door

Suzanne Marjolein Koopmans

geboren op 13 januari 1983 te Tiel 


\section{Promotores}

Prof. dr. H.C. Schouten

Prof. dr. A. zur Hausen

\section{Copromotores}

Dr. A.M.W. van Marion (Viecuri Medisch Centrum, Venlo)

\section{Beoordelingscommissie}

Prof. dr. F. Ramaekers (Voorzitter)

Prof. dr. T. Brümmendorf (Universiteitskliniek, Aken)

Prof. dr. H. van Krieken (Radboud UMC, Nijmegen)

Prof. dr. E.J.M. Speel

Prof. dr. C.D.A. Stehouwer 


\section{CONTENTS}

General introduction

Chapter 1 Myeloproliferative Neoplasia: a review of clinical criteria

and treatment

Neth J Med 2012. may;70(4):159-167

Chapter 2 BCR-ABL negative Myeloproliferative Neoplasia: a review of involved molecular mechanisms

Accepted in Histology and Histopathology

Chapter 3 Reproducibility of histological classification in non-fibrotic myeloproliferative Neoplasia Am J Clin Pathol 2011. oct;136(4):618-624

Chapter 4 The involvement of galectins in the modulation of the JAK/STAT pathway in myeloproliferative neoplasia Am J Blood Res 2012. may;2(2):119-127

Chapter 5 The anti-apoptotic pathways in bone marrow and megakaryocytes in meloproliferative neoplasia.

Pathobiology 2013. Nov;81(2):60-68

Chapter 6 The effect of the JAK2 inhibitor INCB018424 on signalling pathways in Meloproliferative neoplasia; a preliminary study

General discussion

Summary / Samenvatting (summary in Dutch) 


\section{GENERAL INTRODUCTION}

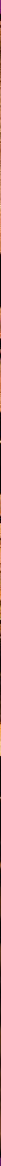




\section{MYELOPROLIFERATIVE NEOPLASIA}

The term "Myeloproliferative Disorders" was first introduced by William Dameshek in 1951, to describe disorders such as Essential Thrombocythemia (ET), Polycythemia Vera (PV), Primary Myelofibrosis (PMF), Chronic Myelogenous Leukemia (CML) and Erythroleukemia (EL). These disorders were grouped together based on their similarities in clinical phenotype and the belief of an underlying undiscovered stimulus responsible for the proliferative activity in these myeloproliferative disorders. ${ }^{[1]}$

Nowadays the term myeloproliferative neoplasia (MPN) is used instead to emphasize the clonal nature of these disorders. According to the World Health Organization (WHO) 2008 criteria, MPNs are now divided into MPNs carrying the Philadelphia $(\mathrm{Ph}+)$ chromosome (CML) and those that do not. ${ }^{[2]}$

In this thesis we focus on the Ph- MPNs which are separated on clinical presentation, laboratory findings and disease course into three different entities; ET, PV and PMF. These three entities are clonal bone marrow stem cell disorders characterized by proliferation of one or more lineages of the myeloid, erythroid or megakaryocytic cell lines. This proliferation results in increased numbers of granulocytes, erythrocytes or platelets in the peripheral blood respectively. ${ }^{[3]}$

Al three entities have in common the risk of thrombotic and haemorrhagic complications. ET is further characterized by a proliferation of the megakaryocytes resulting in increased platelets in the peripheral blood. PV can be recognised by a cellular proliferation of all three lineages with mainly increased erythrocytes as a result. Finally, PMF can be distinguished by the production of megakaryocytes resulting in increased platelets. Furthermore, bone marrow fibrosis can be developed over time. ${ }^{[4]}$ This type of MPN has a higher likelihood of transforming into leukaemia compared to ET and PV patients.

Next to the clinical manifestation and laboratory findings also the bone marrow histomorphology is important in the diagnosis and disease classification of MPN. Characteristic for ET patients are the large, hyperlobulated and mature-appearing megakaryocytes clustered loosely together. Bone marrow of PV patients can be recognised by pleomorphic megakaryocytes which vary in size but without maturing defects. In contrast, megakaryocytes in bone marrow of PMF patients show abnormal maturation and have hyperchromatic and irregularly folded bulky nuclei which are densely clustered. PMF and PV patients have in common that, trilineage myeloproliferation is prominent which is absent in ET patients. Megakaryocyte histomorphology is therefore a very useful tool in disease classification to distinguish MPN from other related myeloid malignancies. Furthermore, reticulin and collagen 
fibrosis and often also osteosclerosis are present in fibrotic PMF. Low grades of reticulin fibrosis are sometimes also found in the bone marrow of ET and PV patients. In general, bone marrow in MPN display trilineage myeloid hyperplasia, megakaryocyte clusters and develop eventually reticulin fibrosis. ${ }^{[5-6]}$

It is well known that ET patients experience fewer complications. However, approximately less than $10 \%$ of patients will develop myelofibrosis during their disease course, known as post-ET myelofibrosis, which is associated with a worse prognosis. Less than $2 \%$ of the ET patients progress to acute myeloid leukaemia(AML). Of the PV patients approximately $30 \%$ will develop post-PV myelofibrosis and leukemic transformation will occur in about $1 \%$ of the cases. Patients with prefibrotic PMF have few complaints, however during the course of the disease the degree of reticulin increases in the bone marrow and spleen resulting in collagen fibrosis with osteosclerosis and decreased haematopoiesis. The clinical picture for these patients is determined by the degree of anaemia and splenomegaly. Leukemic transformation occurs in $10 \%$ of the PMF patient population. ${ }^{[7-9]}$

Bone marrow biopsies are one of the most important instruments in MPN diagnostics and are carried out for several purposes. Firstly, to diagnose MPN and if possible the classification of MPN in ET, PV or PMF. Secondly, to evaluate the histologic criteria for prognostic significance. And, finally, to gain more insight in the pathogenesis of MPN development. This may also serve as basis for the development of novel therapies.

The determination of the prognostic parameters is very important for the patients. However, prediction of the disease course, and therefore the prognosis, is difficult. There is growing evidence that the marrow microenvironment can contribute to inducing and sustaining haematological malignancies. The development of fibrosis in the bone marrow of MPN patients is an important negative prognostic factor and determines the clinical course and survival chances. Unfortunately, the underlying mechanisms are still not defined and no therapeutic options exist, other than transplantation, to prevent the fibrosis development or stop its progression.

The discovery of the JAK2 ${ }^{V 617 F}$ mutation has generated more insight in the pathogenesis of MPN development. The JAK2 ${ }^{\mathrm{V} 617 F}$ mutation leads to a sustained JAK2 activation resulting in increased phosphorylation of the extracellular signal-regulated kinase signalling pathway, phosphatidylinositol-3-kinase (PI3K)/Akt pathway and activation of the signal transducer and activator of transcription (STAT) family. ${ }^{[10,11]}$

Although the discovery of the JAK2 ${ }^{\mathrm{V} 617 F}$ mutation is of paramount importance to gain more insight in the MPN development it is increasingly becoming clear that the currently discovered mutations in MPN patients are secondary events. In other words the JAK2 ${ }^{\mathrm{V} 617 F}$ 
mutation is not the underlying pathogenetic cause of MPN. The objectives of this thesis are to gain better insight in the pathogenesis of the myeloproliferative neoplasia and some of the molecular parameters in the development of MPN. We first assess the current major individual morphological characteristics described in the WHO classification and the reproducibility of the histological MPN diagnosis. Next we aim to determine the importance of different oncogenic pathways involved in the mechanism of megakaryocytic differentiation and apoptosis. In addition, we assess in as much the development of fibrosis and angiogenesis, might be used for the future development of new therapeutic strategies. 


\section{REFERENCES}

1. Dameshek, W., Some speculations on the myeloproliferative syndromes. Blood, 1951. 6(4): p. 372-5.

2. Vardiman, J.W., Thiele, J., Arber, D.A., et al., The 2008 revision of the World Health Organization (WHO) classification of myeloid neoplasms and acute leukemia: rationale and important changes. Blood, 2009. 114(5): p. 937-51.

3. Campbell, P.J., Green, A.R., The myeloproliferative disorders. N Engl J Med, 2006. 355(23): p. 2452-66.

4. Murray, J., Myeloproliferative disorders. Clin Med, 2005. 5(4): p. 328-32.

5. Michiels, J.J., et al., Current diagnostic criteria for the chronic myeloproliferative disorders (MPD) essential thrombocythemia (ET), polycythemia vera (PV) and chronic idiopathic myelofibrosis (CIMF). Pathol Biol (Paris), 2007. 55(2): p. 92-104.

6. Thiele, J., Philadelphia chromosome negative chronic myeloproliferative disease. Am J Clin Pathol, 2009. 132(2): p.261-80.

7. Thiele, J., Kvasnicka, H.M., Fischer, R., Histochemistry and morphometry on bone marrow biopsies in chronic myeloproliferative disorders - aids to diagnosis and classification. Ann Hematol, 1999. 78(11): p. 495-506.

8. Murray, J., Myeloproliferative disorders. Clin Med, 2005. 5(4): p. 328-32.

9. Tefferi, A., How I treat myelofibrosis. Blood, 117(13): p. 3494-504.

10. James, C., et al., A unique clonal JAK2 mutation leading to constitutive signalling causes polycythaemia vera. Nature, 2005. 434(7037): p. 1144-8.

11. Dhillon, A.S., et al., MAP kinase signalling pathways in cancer. Oncogene, 2007. 26(22): p. 3279-90. 



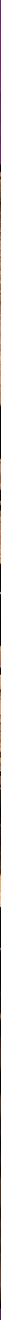

Neth J Med 2012. may;70(4):159-167 


\section{ABSTRACT}

Essential thrombocythemia (ET), polycythemia vera (PV) and primary myelofibrosis (PMF) belong to the group of Philadelphia chromosome negative Myeloproliferative Neoplasia (PhMPN). MPNs are clonal bone marrow stem cell disorders characterized by a proliferation of one or more of the myeloid, erythroid or megakaryocytic cell lines. Due to the different affected cell lines, the MPNs show typical clinical and histological features. In 2005 a mutation in the JAK2 gene was discovered which generated more insight in the pathogenetic working mechanism of the MPNs. However, the treatment of MPN patients is still mainly only palliative but progresses in reducing the symptoms of MPN patients have been made. This review will give a general overview of MPN patients for internal medicine physicians. 


\section{HAEMATOPOIESIS}

Haematopoiesis is the development of the cellular components of the blood. The formation and development of blood cells is initiated by the haematopoietic stem cells (HSCs). HSCs are primitive cells capable of self renewal and differentiation. Due to the self renewal capability, at least one of the daughter cells possesses the same HSC characteristics as the mother cell after cell division. During the entire life of an individual, the stem cell pool is maintained due to the self renewal capability of the HSCs and supplies cells for multilineage haematopoiesis. ${ }^{[1,2]}$ Currently it is considered that long-term repopulating HSC (LT-HSC) differentiate into a short-term repopulating HSC (ST-HSC) and as schematically shown in figure 1, it will differentiate further in multipotent progenitor cells (MPP) only capable of differentiating into the myeloid lineage or the lymphoid lineage. The common myeloid progenitors (CMP) give rise to megakaryocyte-erythroid progenitors (MEP), which differentiate into megakaryocytes and erythrocytes, and granulocyte-monocyte progenitors (GMP), which differentiate into macrophages and neutrophil granulocytes. The eosinophilic and basophilic granulocytes differentiate directly from the CMP. The common lymphoid progenitors (CLP) differentiate into T- and B-lymphoid cells and natural killer cells (see Figure 1). The progeny that arises from HSCs loose progressively their self renewal capacity and becomes gradually more restricted to one lineage. ${ }^{[3,4]}$

HSCs require intrinsic and extrinsic factors for their activities provided by the stem cell niche. The interaction of the HSCs with the stem cell niche determines whether the HSCs remain in a quiescent state or proliferate to progenitor cells and differentiate into mature blood cells. ${ }^{[5,6]}$ 
Figure 1: Development of haematopoietic stem cells, a schematic view.

Abbrevations: HSC haematopoietic stem cells; LT-HSC long-term repopulating HSC; ST-HSC short-term repopulating HSC; MPP multipotent progenitor; CMP common myeloid progenitor; MEP megakaryocyteerythroid progenitor; GMP granulocyte-macrophage progenitor; CLP common lymphoid progenitor.

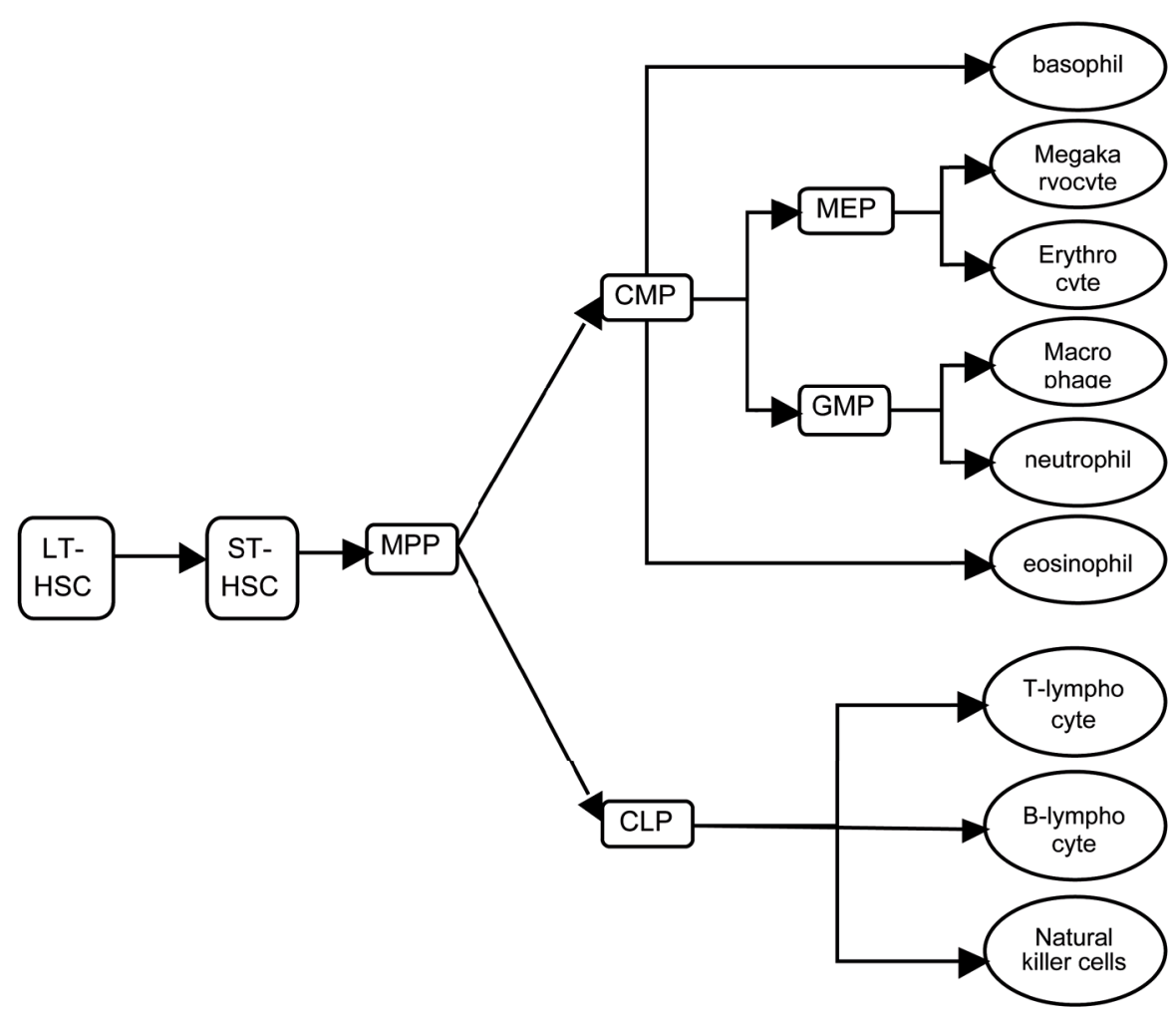




\section{MYELOPROLIFERATIVE NEOPLASIA}

Myeloproliferative neoplasia (MPNs) are clonal bone marrow stem cell disorders involving a multipotent haematopoietic stem cell, characterized by proliferation of one or more lineages of the myeloid, erythroid and megakaryocytic cell lines. This proliferation results in increased numbers of granulocytes, erythrocytes or platelets in the peripheral blood respectively. ${ }^{[7]}$ William Dameshek was the first who introduced the term "myeloproliferative disorders" in 1951 including essential thrombocythemia (ET), polycythemia vera (PV), primary myelofibrosis (PMF), chronic myelogenous leukaemia (CML) and erythroleukaemia (Di Guglielmo syndrome). These disorders were grouped together based on their similarities in clinical phenotype and the believe of an underlying undiscovered stimulus responsible for the proliferative activity of bone marrow cells in these myeloproliferative disorders. ${ }^{[8]}$ According to the World Health Organization (WHO) 2008 criteria, MPNs are now divided in classical MPNs which carry the Philadelphia ( $\mathrm{Ph}+$ ) chromosome (Chronic Myeloid Leukaemia) and classical MPNs which do not carry the Philadelphia (Ph-) chromosome including ET, PV and PMF. The Philadelphia chromosome is a result of $\mathrm{t}(9: 22)$ with the BCR-ABL1 fusion gene. ${ }^{[9]}$ In this article the classical Ph- MPNs are highlighted.

\section{Clinical and histological criteria of MPN}

The typical features of ET are thrombotic and haemorrhagic complications, although most patients are asymptomatic. Transient ischemic attacks, erythromelalgia and Budd-Chiari syndrome are complications which can occur in ET patients or can occur before the diagnosis ET is apparent. Bleeding complications are a result of an extremely high platelet count resulting in an acquired von Willebrand disease; von Willebrand factor will be proteolysed with increasing platelet counts. ${ }^{[10]}$ Histomorphological findings in the bone marrow of ET patients are loose clusters of predominant large to giant megakaryocytes. The megakaryocytes exhibit a normal maturation with hyperlobulated and staghorn-like nuclei (see Figure 2). There is no marked left-shifting of the erythroid or myeloid cell line apparent. The presence of reticulin is extremely rare in ET patients at presentation and very few patients $(<10 \%)$ develop myelofibrosis during their disease course, known as post-ET myelofibrosis. ET patients have a risk of approximately 2\% to develop Acute Myeloid Leukaemia (AML). ${ }^{[11,12]}$

Polycythemia vera is characterized by a trilineage proliferation of the erythroid, myeloid and megakaryocytic cell line, usually resulting in mainly increased erythrocytes and often also leukocytes and blood platelets. Patients also display a persistently raised haemoglobin and haematocrit level. The clinical features of PV patients are vascular occlusive events, enlarged spleen, aquagenic pruritus (intense itching after a hot bath or shower) and haemorrhagic complications after injuries and surgery. In about $30 \%$ of the patients PV will develop 
myelofibrosis, known as post-PV myelofibrosis, and leukemic transformation will occur in about $10 \%$ of the PV patients. ${ }^{[12]}$ The bone marrow of PV patients displays panmyelosis and therefore an increase in cellularity. The megakaryocytes reveal a range from small to giant megakaryocytes without maturation defects of nuclei and cytoplasm and are arranged in loose clusters (see Figure 2). There is always a proliferation and often a left-shifting of the myeloid cell lineage and especially of the erythroid precursor cells. Slightly increased reticulin fibrosis can be seen in the bone marrow. ${ }^{[11]}$

In primary myelofibrosis the patient's complaints and symptoms depend mainly on the degree of anaemia and splenomegaly. The typical early symptoms are fatigue, weight loss, night sweating and fever. These constitutional symptoms are believed to be mediated by the abnormal release of cytokines from clonal megakaryocytes as a result of emperipolesis. When the fibrosis is in an advanced stage, the complaints are, apart from the constitutional symptoms, paleness due to anaemia, hepatosplenomegaly, spleen infarct and osteosclerosis. Budd-Chiari syndrome can be a feature of early-phase disease and can be the presenting symptom. ${ }^{[12,13]}$ In the bone marrow of prefibrotic PMF an overall hypercellularity is evident including prominent growth of abnormally differentiated and giant megakaryocytes. The megakaryocytes reveal hypolobulated, cloud-like and hyperchromatic nuclei and demonstrate dense clustering (see Figure 2), often accompanied by left-shifted granulocyte proliferation. In the prefibrotic PMF reticulin fibrosis may be absent, but during the disease course reticulin fibrosis increases, finally resulting in collagen fibrosis with osteosclerosis. Leukemic transformation occurs in about $10 \%$ of the PMF patients. ${ }^{[14]}$

However, the symptoms listed above are not strictly limited to ET or PV or PMF patients, in fact they can occur in all three classical Ph- MPN, such as bleeding complications Ispontaneous or after surgeryl, thrombosis and fatigue. MPN patients may even be asymptomatic in the early-phases of the disease and it may be a coincidence that a MPN disease is discovered by abnormal blood counts or by diseases which are features of early-phase MPN, like BuddChiari syndrome, heart attack, cerebral vascular accident, pulmonary thrombus and deep venous thrombosis. An important factor in thromboembolic events is the JAK2 $2^{\text {V617F }}$ mutation. No differences in thromboembolic events were seen between heterozygous and homozygous

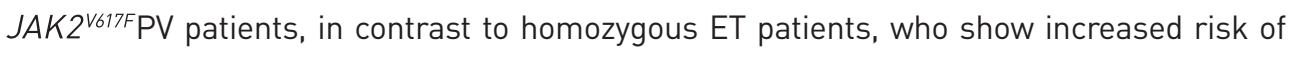
cardiovascular events compared to heterozygous and wild-type ET patients. It was also shown that ET and PV patients with a higher allele burden have a higher risk of thrombotic events. ${ }^{[15]}$

This indicates an important risk factor for the JAK2 $2^{\mathrm{V} 617 \mathrm{~F}}$ mutation in the development of thrombosis. The JAK2 $2^{\mathrm{V} 617 F}$ occurrence rate in patients with thrombosis of the deep veins (DVT) and pulmonary embolism (PE) is low, therefore a general JAK2 ${ }^{\text {V617F }}$ screening is not recommended among patients with spontaneous DVT and PE. This is in contrast to patients 
who present with splanchnic and intrahepatic vein trombosis, these patients show a high prevalence of the JAK2 ${ }^{\text {V617F }}$ mutation and a diagnosis of ET or PV should be kept in mind. ${ }^{[16,17]}$

Figure 2: Examples of morphological features in megakaryocytes.

A. Dense clustering (HE, 630x) B. Loose clustering (HE, 1000x) C. Dysmorphic nucleus (HE, 1000x)

D. Hyperlobulated nucleus (HE, 1000x) E. Staghorn nucleus (HE, 1000x) F. Cloud-like nucleus (HE, 1000x).
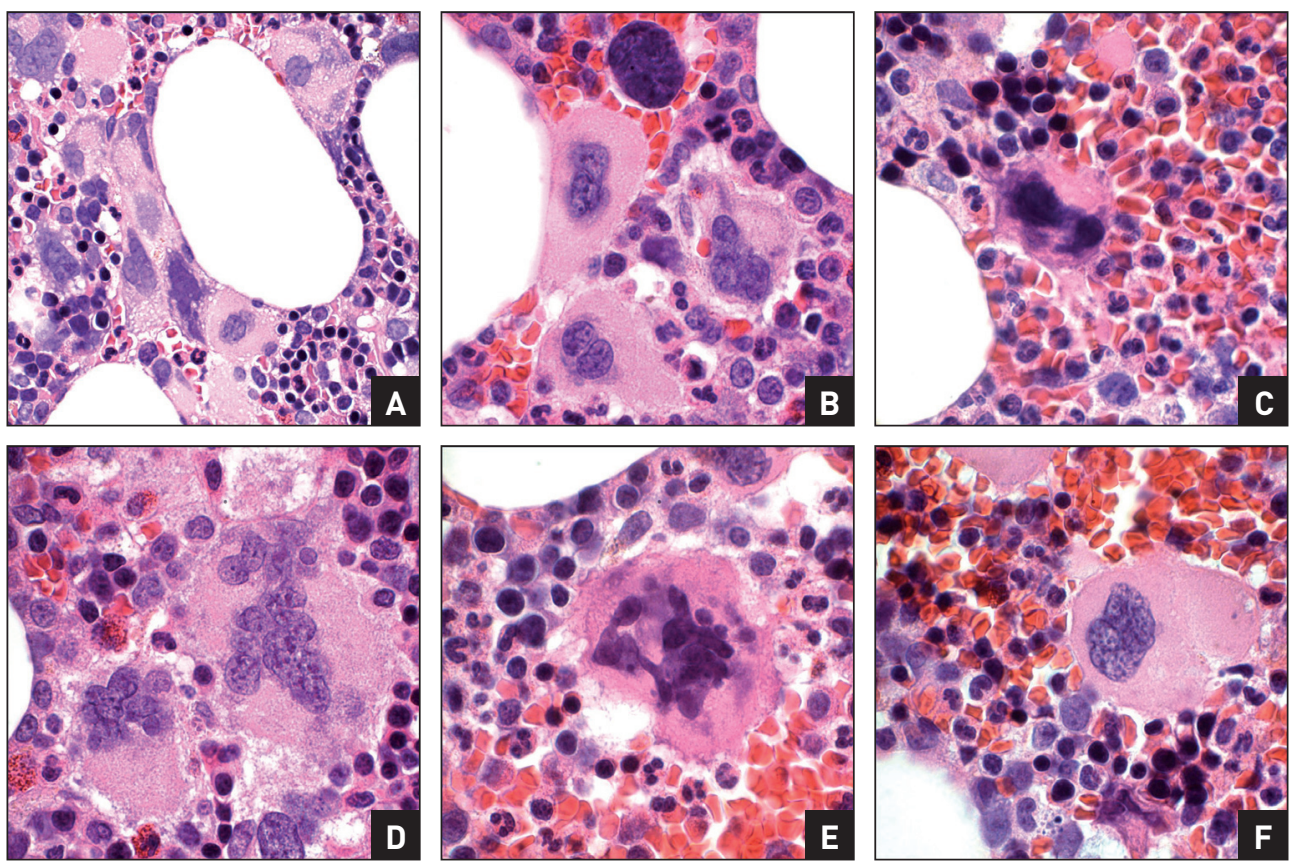

The Polycythemia Vera Study Group (PVSG) made the first attempt in establishing diagnostic criteria for the Ph-MPNs in 1967. The diagnostic criteria were updated several times during the following decades and are even now widely used by haematologists. However, the appropriate use of bone marrow biopsy (BMB) histology as a diagnostic tool was neglected. To stress the relevance of a BMB, the WHO added a set of histological diagnostic criteria in 2001. The recent discovery of the JAK2 ${ }^{\text {V617F }}$ mutation and the recognition of pre-fibrotic PMF resulted in the 2008 WHO classification of MPNs. ${ }^{[18-20]}$ However, the early-phases of ET, PV and PMF are difficult to distinguish on morphology alone as they share many morphological characteristics. It was shown by Wilkins et al. that some of the histological criteria as described in the WHO classification were difficult to reproduce. ${ }^{[21]}$ Nevertheless, it is very important to distinguish these three MPN subtypes in the early-phase reliably, because of a different risk of thromboembolic complications of PV and the worse survival rate of PMF patients compared to ET patients, who have a normal life expectancy. ${ }^{[21,22]}$ 
Although Ph- MPNs are divided in three clinical distinct entities, the use of three distinct diagnoses can also be questioned; ET, PV and PMF show a great abundance of overlap in their morphological characteristics, clinical signs and symptoms and can also share the same molecular mutation (JAK2 ${ }^{\mathrm{V} 617 \mathrm{~F}}$ ). A proposed simplistic model for revision of the MPN classification is shown in Figure 3. It might be more reasonable to divide the MPNs in JAK2 positive and negative diseases and subdivide them in patients with and witho ut myelofibrosis. ${ }^{[23]}$

Figure 3: Proposed model for reconsidering the classification of MPNs.

Abbrevations: MPN Myeloproliferative Neoplasia; JAK2+ positive for the JAK2 ${ }^{\mathrm{V} 617 \mathrm{~F}}$ mutation; JAK2wild type JAK2; MF+ myelofibrosis present in the bone marrow; MF- myelofibrosis absent in the bone marrow.

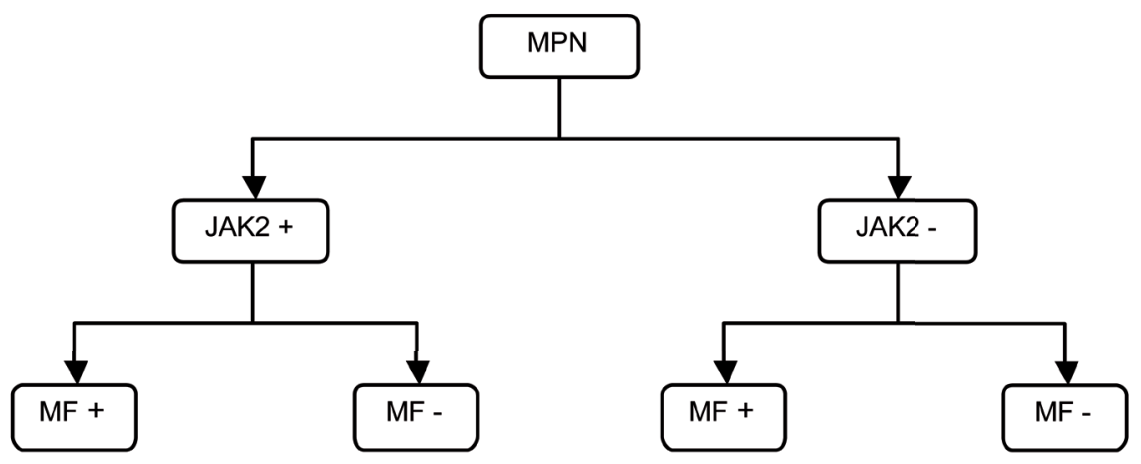

\section{THE JAK2 MUTATION AND MPN}

In 2005 several groups identified a mutation in the tyrosine kinase domain of JAK2 in MPN patients, resulting in a substitution of valine for phenylalanine at position 617 of

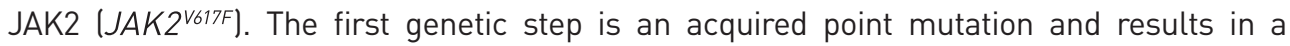
heterozygous mutational status. The homozygous JAK2 $2^{\text {V617F }}$ mutation is the result of mitotic recombination between homologous chromosomes $9 p$ and results in loss of heterogeneity of $9 p(\mathrm{LOH})$ and is a second genetic step in the aetiology of the MPNs. ${ }^{[2-28]}$ The JAK2 ${ }^{\mathrm{V} 617 \mathrm{~F}}$ mutation is present in granulocytes, erythroblasts and myeloblasts and in all erythropoietin (EPO)-independent erythroid colonies. The erythroid colonies with the $J A K 2^{\mathrm{V} 617 F}$ mutation are able to grow in the absence of EPO. Therefore, the JAK2 ${ }^{\mathrm{V} 617 F}$ mutation results also in factor independent growth of various haematopoietic cell lines. ${ }^{[29]}$ Further, the receptors of bone marrow progenitor cells are hypersensitive to thrombopoietin (TPO, stimulates proliferation and differentiation of megakaryocytes), EPO (stimulates erythroblasts), stem cell factor (SCF, induces proliferation and self-renewal of multipotent 
haematopoietic progenitors) and granulocyte stimulating factor (GSF, stimulates proliferation and differentiation of granulocytes). The hypersensitivity for these cytokines results in specific stimulation of the megakaryopoiesis, erythropoiesis and granulopoiesis. ${ }^{[30]}$

The JAK2 ${ }^{\mathrm{V} 617 \mathrm{~F}}$ mutation is present in $>95 \%$ of the PV patients and in approximately $50 \%$ of the ET and PMF patients. ${ }^{[15,31]}$ The JAK2 ${ }^{\mathrm{V} 617 F}$ mutation deregulates the JAK2 kinase activity. The mutation is located in the JH2 domain of the JAK2 gene, which negatively regulates the activity of the kinase domain, JH1. Valine 617 and cysteine 618 maintain both the kinase domain of JAK2 in an inactive state. Substition of valine 617 for phenylalanine destabilizes this inhibitory interaction, resulting in increased JAK2 kinase activity. Altogether, this suggests that there is a sustained JAK2 activation, while the feedback mechanism has been destroyed with a growth factor independent activation. ${ }^{[24]} \mathrm{PV}$ patients without the JAK2 ${ }^{\mathrm{V} 617 F}$ mutation have virtually all a JAK2 ${ }^{\mathrm{V} 617 F}$ exon 12 mutation. Also more early genetic abnormalities are currently being defined and related with disease development.

\section{TREATMENT OF MPN}

The current treatment of MPN patients is mostly supportive, while standard therapy has not been defined firmly. The treatment of ET and PV patients should be done according to their risk stratification for the occurrence of thromboembolic processes (see Table 1 and Table 2) as evaluated in a large prospective study of the European Collaboration on Low-dose Aspirin in Polycythemia (ECLAP). ${ }^{[32]}$ Age greater than 60 years and a previous history of thrombosis were found to be risk factors for thrombosis in both ET and PV. Is one of both criteria present, the ET and PV patient is at high-risk, whereas if none of the criteria are present ET and PV patients are at low-risk. ET and PV patients who have platelets $>1000^{*} 10^{\circ} / \mathrm{L}$ are of intermediate risk to develop thrombosis or if they have any of the following risk factors; hypertension, hypercholesterolemia, smoking and diabetes mellitus (see Table 1). These are generic cardiovascular risk factors, and their role is still controversial. Other possible risk factors, which have to be validated in prospective studies, might be leukocytosis and

Table 1: Risk-stratification of patients with ET and PV for the occurrence of thrombosis.

\begin{tabular}{|l|l|l|}
\hline \multicolumn{1}{|c|}{ Risk category } & \multicolumn{1}{|c|}{$\begin{array}{c}\text { Age }>60 \text { years or } \\
\text { history of thrombosis }\end{array}$} & \multicolumn{1}{c|}{$\begin{array}{c}\text { Generic cardiovascular } \\
\text { risk factors }\end{array}$} \\
\hline Low & No/No & Yes \\
\hline Intermediate & Platelets $>1000^{*} 10^{\circ} / \mathrm{L}$ & Irrelevant \\
\hline High & Yes/No or No/Yes & \\
\hline
\end{tabular}


the presence of the JAK2 $2^{\text {617F }}$ mutation, although the latter is controversial. ET patients belonging to the low-risk or intermediate-risk category and without any symptoms do not need therapy, however, aspirin is recommended to prevent microvascular disturbances as erythromelalgia, although major bleeding or presence of von Willebrand syndrome are contraindications for the use of aspirin. High-risk ET is an indication for the use of hydroxyurea (HU), which inhibits thrombocyte-, erythrocyte- and leukocyte production, combined with low-dose aspirin if thrombosis or microvascular symptoms are present,

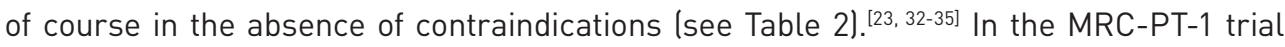
researchers compared $\mathrm{HU}$ plus aspirin with anagrelide plus aspirin in ET patients at high risk for thrombosis, observing that $\mathrm{HU}$ plus low-dose aspirin is superior to anagrelide plus low-dose of aspirin. ${ }^{[36]}$

The administration of aspirin to PV patients has been widely investigated. In 1986 the PVSG concluded that aspirin was ineffective and dangerous, due to increased gastrointestinal bleeding and intra-cerebral haemorrhage, based on a randomized trail of 163 PV patients receiving either $900 \mathrm{mg} / \mathrm{d}$ aspirin plus dipyridamole or radioactive phosphorus ( $\left.{ }^{32} \mathrm{P}\right) .{ }^{[37]}$ However, more studies on the administration of aspirin have been done, resulting in the conclusion of the safe use of a considerably lower dose of aspirin in PV patients. The Gruppo Italiano Studio Policitemia Vera demonstrated the safe use of a low-dose aspirin $(40 \mathrm{mg} / \mathrm{d})$ in PV patients. ${ }^{[38]}$ The study by Landolfi et al. ${ }^{[39]}$ showed a significant reduction in the combined risk of cardiovascular death, nonfatal myocardial infarction, nonfatal stroke, pulmonary embolism or major venous thrombosis with $100 \mathrm{mg} / \mathrm{d}$ of aspirin. Therefore, low-dose of aspirin plus phlebotomies are recommended in the low-risk and intermediate-risk category. ${ }^{[23]}$

In 1953 the most effective treatment of PV included phlebotomies combined with radioactive phosphorus $\left({ }^{32} \mathrm{P}\right)$ resulting in prolonged survival, however ${ }^{32} \mathrm{P}$ was shown to be leukemogenic. ${ }^{[40]}$ The PVSG study group conducted a randomized trial comparing phlebotomy alone with ${ }^{32} \mathrm{P}$ plus phlebotomy and with chlorambucil plus phlebotomy. Patients treated with phlebotomy alone showed a higher incidence of thrombosis in the first three years of treatment. After three to five years of study, a considerably number of patients treated with ${ }^{32} \mathrm{P}$ or chlorambucil developed acute leukaemia, lymphoma and carcinomas of the gastrointestinal tract and skin, compared to those treated with phlebotomy alone. Therefore, patients treated with phlebotomy alone had a better overall median survival of 13.9 years than patients treated with chlorambucil (8.9 years) or ${ }^{32} \mathrm{P}$ (11.8 years). ${ }^{[33]}$ The PVSG also compared HU with phlebotomy, a slightly higher incidence of acute leukaemia, less myelofibrosis and fewer deaths among the patients treated with HU were apparent. ${ }^{[4]}$ Interferon- $\alpha$ is able to inhibit in vitro proliferation of haematopoietic progenitors and inhibition of the thrombopoietin-induced MPL receptor signalling resulting in megakaryopoiesis repression. The use of IFN- $\alpha$ in PV patients was shown to be effective 
and non-leukemogenic. However, the use of IFN- $\alpha$ has been limited due to its toxicity, parenteral administration and costs. ${ }^{[42,43]}$ The development of pegylated (peg) forms of IFN resulted in improved tolerance, efficacy and fewer side effects. ${ }^{[44,45]}$ Peg-IFN- $\alpha$ has been demonstrated to have clinical advantages, high rates of molecular response and lower toxicity in phase II trials in PV as well as ET patients. ${ }^{[46,47]}$

PV patients belonging to the low-risk or intermediate-risk category with high haematocrit level are treated with phlebotomies in order to obtain normal haematocrit levels $(<0.45 \mathrm{~L} / \mathrm{L})$ plus low dose aspirin, if no contraindications are present. If $P V$ patients show poor compliance to phlebotomy or if they show progressive myeloproliferation cytoreductive therapy should be given. The high-risk group should be treated with myelosuppression, with HU as the drug of choice (see Table 2).

Table 2: Treatment of ET and PV according to their risk stratification.

\begin{tabular}{|l|l|l|}
\hline Risk category & \multicolumn{1}{c|}{ ET } & \multicolumn{1}{c|}{ PV } \\
\hline Low & $\begin{array}{l}\text { Low-dose aspirin* } \\
\text { if microvascular } \\
\text { disturbances are present }\end{array}$ & $\begin{array}{l}\text { Phlebotomie + } \\
\text { low dose aspirin* }\end{array}$ \\
\hline Intermediate & $\begin{array}{l}\text { Low-dose aspirin* } \\
\text { if microvascular } \\
\text { disturbances are present }\end{array}$ & $\begin{array}{l}\text { Phlebotomie + } \\
\text { low dose aspirin }\end{array}$ \\
\hline High & $\begin{array}{l}\text { Low-dose aspirin* } \\
\text { if microvascular disturbances } \\
\text { are present + hydroxyurea^ }\end{array}$ & $\begin{array}{l}\text { Phlebotomie + } \\
\text { low dose aspirin } \\
+ \text { hydroxyurea }\end{array}$ \\
\hline
\end{tabular}

* In the case of major bleeding or presence of von Willebrand syndrome, aspirin is a contraindication

$\wedge$ hydroxyurea-intolerance or -resistance, use anagrelide or peg-INF- $\alpha$

Anagrelide or peg-INF- $\alpha$ is used in PV and ET patients in case of intolerance or resistance to $\mathrm{HU}$, to control platelet count or who develop side effects to HU, however long-term efficacy and safety features are still unknown. ${ }^{[23,35,48]}$ The prognosis of PMF patients is worse than of ET or PV patients (median survival six versus twenty years) and the disease course is not significantly modified by drug therapy, therefore treatment of PMF is mainly palliative. However, there is a wide heterogeneity in presentation and evolution among PMF patients. Therefore, the International Prognostic Scoring System (IPSS) uses five risk factors for estimating the survival of PMF patients at time of diagnosis: age $>65$ years, constitutional symptoms (weight loss, fever, excessive sweating), haemoglobin level $<10 \mathrm{~g} / \mathrm{dL}$, leukocyte count $>25^{*} 10^{\%} / \mathrm{L}$ and circulating blasts $>1 \%$. Based on this system PMF patients can be categorized in low risk group (0 risk factors present), intermediate-1 (1 risk factor present), intermediate-2 (2 risk factors present) and high risk group ( $\geq 3$ risk factors present). ${ }^{[49]}$ 
IPSS has been modified to Dynamic IPSS (DIPSS) with the same five risk factors to estimate the survival during the disease course, while acquisition of additional risk factors modifies the patients' outcome. ${ }^{[50]}$ Recently, the DIPSS was upgraded to DIPSS-plus by incorporating three independent prognostic factors, including the need for red cell transfusion, thrombocytopenia $<100 * 10^{\%} / \mathrm{L}$ and unfavourable karyotype (including $+8,-7 / 7 q-, i(17 q),-5 / 5 q-, 12 p-$, inv(3) or $11 \mathrm{q} 23$ rearrangement). Based on the DIPSS-plus PMF patients are categorized in low (no risk factors), intermediate-1 (1 risk factor), intermediate-2 (2 or 3 risk factors) and high ( $\geq 4$ risk factors) risk group. Unfavourable karyotype and thrombocytopenia both predict leukemic transformation in PMF patients. If the patient needs red cell transfusion, the patient belongs to the intermediate risk group, while the patient displays 2 risk factors: anaemia and red cell transfusion need. ${ }^{[51]}$

A wait-and-see approach is justified in PMF patients belonging to the low- or intermediate-1 risk group, while the median survival of these patients exceeds 15 and 6 years respectively. ${ }^{[1]}$ This relative long median survival does not justify the risks of an allogeneic stem cell transplantation (alloSCT) or the start of investigational drug therapy. There is also no evidence to support the use of conventional drug therapy in low- or intermediate-1 risk group patients if the patients do not have complaints which can be treated (see Table 3). ${ }^{[13]}$ However, if PMF patients suffer from splenomegaly, the first drug of choice is $\mathrm{HU}$ and in the worst case splenectomy is indicated. Indications for splenectomy include symptomatic portal hypertension, drug-refractory splenomegaly with severe symptoms, transfusiondependent anaemia, marked thrombocytopenia and uncontrollable haemolysis due to severe complications that can occur. Irradiation therapy of the spleen transiently reduces spleen size and reduces the incidence of pancytopenia. Patients usually experience relief of constitutional symptoms when splenomegaly is treated. In the case of non-hepatosplenic extramedullary haematopoiesis (located in mainly the thoracic vertebral column or in lymph nodes, lung pleura, small bowel, peritoneum, urogenital tract and heart) low-dose irradiation therapy is indicated. ${ }^{[52,53]}$

Patients belonging to the intermediate-1 risk group who suffer from the risk factor they display, conventional drug therapy should be given; anaemia can be treated with androgens, danazol, corticosteroids, thalidomide or lenalidomide. Thalidomide plus prednisone and lenalidomide plus prednisone show higher response rates with decreased toxicity. Thalidomide and lenalidomide are also effective in PMF patients with unfavourable karyotype. A recent study of Holle et al. ${ }^{[54]}$ showed an improvement in haemoglobin and thrombocyte counts and a reduction in spleen size and bone marrow fibrosis in patients with PMF, post-ET and post-PV myelofibrosis treated with thalidomide. However, side effects are toxicity and mainly neurotoxicity. More promising might be lenalidomide which shows fewer side effects with similar improvement in haematopoiesis. ${ }^{[53]}$ The use 
of erythropoiesis-stimulating agents in myelofibrosis is not recommended due to the risk of splenomegaly exacerbation. ${ }^{[52,55]}$ PMF patients in the intermediate-2 and high risk group have an indication for therapy, as well as regular therapy as investigational drug therapy, due to the low survival rates in these patients (see Table 3).

Table 3: Treatment of PMF according to their risk stratification.

\begin{tabular}{|l|l|}
\hline Risk category & \multicolumn{1}{c|}{ PMF } \\
\hline Low & Wait-and-see or conventional drug therapy \\
\hline Intermediate-1 & Wait-and-see or conventional drug therapy \\
\hline Intermediate-2 & Hydroxyurea* or experimental drugs or alloSCT \\
\hline High & Hydroxyurea* or experimental drugs or alloSCT \\
\hline
\end{tabular}

*Hydroxyurea-intolerance or -resistance, use peg-INF-a

In the presence of thrombocytosis, leukocytosis, splenomegaly or bone pain, there is an indication for hydroxyurea. Anaemia can be treated as indicated for the intermediate-1 risk group and splenectomy is also indicated as stated above. ${ }^{[23,53,56-60]}$

The only potentially curative treatment in PMF patients is allogeneic stem cell transplantation with an overall three years survival ranging from 30-60\%. AlloSCT can induce Graft versus Host Disease (GvHD), which can be divided in acute GvHD and chronic GvHD, with an incidence of about $30-43 \%$ and $30-48 \%$ respectively. ${ }^{[61-63]}$ However, despite the high rate of death and the high risk of chronic morbidity due to GvHD, alloSCT is justified in PMF patients belonging to the intermediate-2 or high risk group, while the median survival of these patients are 3 years and 1 year ${ }^{[64]}$ respectively (see Table 3 ). The three-year overall survival of PMF patients after alloSCT is ranging from $37 \%$ to $58 \%$.

\section{FUTURE TREATMENT}

New therapeutical strategies include JAK inhibitors and imatinib mesylate. Imatinib mesylate (tyrosine kinase inhibitor) is used in the treatment of chronic myelogenous leukaemia and has been shown to reduce spleen size and to reduce the proliferative activity in PV patients. ${ }^{[65]}$ Several JAK inhibitors have been developed since the discovery of the JAK2 ${ }^{\text {V617F }}$ mutation in 2005, among them Ruxolitinib (INCB018424), SAR302503 (TG101348), CYT387, Lestaurtinib (CEP701) and SB1518. 
Ruxolitinib is a JAK1 and JAK2 inhibitor which was tested in a phase I/II trial. Patients showed responses after one to two months including reduction of spleen size and improvement of constitutional symptoms including fatigue, weight loss, night sweats and pruritus. More than $50 \%$ decrease in total symptom score after 24 weeks occurred in $46 \%$ of the patients compared to $5 \%$ for the placebo group. Haematological side effects were anaemia and thrombocytopenia (grade 3 or 4). Non-haematological toxic effects were of low grade and infrequent. After 60 days the overall survival of the patients treated with ruxolitinib was higher compared to the placebo group (hazard ratio $=0.67$ ). Allele burden was minimally decreased and ruxolitinib was shown to be effective in patients with the JAK2 $2^{\mathrm{V} 617 F}$ mutation, but also in patients without the JAK2 mutation. ${ }^{[66,67]}$ Ruxolitinib is now tested in a phase III trial.

In a recent study of Tefferi et al. 51 patients were enrolled in the phase I/II COMFORT trial experiencing a very rapid relief of symptoms related to the presence of myelofibrosis and splenomegaly. However, the occurrence of serious anaemia and thrombocytopenia, loss or lack of response, disease progression, patient/physician choice often associated with lack of response, and death during study prompted 47 patients to discontinue with ruxolitinib treatment. During treatment discontinuation acute relapse of symptoms and splenomegaly were experienced by most patients with sometimes hospitalization requirement. This observation stresses the need for careful disclosure of the ruxolitinib withdrawal syndrome to myelofibrosis patients. Further, treatment discontinuation should be done under close supervision in a gradual tapering schedule, although the tapering schedule does not guarantee that the withdrawal symptoms will not occur. ${ }^{[68]}$ However, these side effects and the occurrence of ruxolitinib withdrawal syndrome do not counteract the benefits MPN patients with myelofibrosis experience with ruxolitinib treatment.

SAR302503 is a selective JAK2 inhibitor inducing rapid spleen size reduction and improvement of constitutional symptoms. Further, the majority of patients with leukocytosis and thrombocytosis at baseline achieved normal blood counts. A significant decrease in the $J A K 2^{V 617 F}$ allele burden was observed. Grade 1 self limiting side effects were nausea, diarrhoea and vomiting. Haematological side effects of grade 3 to 4 were anaemia, thrombocytopenia and less frequent neutropenia. ${ }^{[69]}$ SAR302503 is at the moment tested in a phase II trial.

CYT387 inhibits the JAK1 and JAK2 gene. First results are promising; improvement in spleen size, anaemia and constitutional symptoms. Side effects were headache and thrombocytopenia. ${ }^{[70]}$ CYT387 is currently under investigation in a phase I/II trial.

Lestaurtinib inhibits JAK2 and JAK3 and improves spleen size, transfusion dependency and cytopenias. No effect was seen on the JAK2 ${ }^{\mathrm{V} 617 F}$ allele burden. Side effects were diarrhoea, anaemia and thrombocytopenia. ${ }^{[71]}$ Currently, lestaurtinib is under investigation in a phase II trial. SB1518 is a highly selective JAK2 inhibitor and was well tolerated in a phase I trial with a decrease in spleen size and improvement in clinical symptoms. ${ }^{[72]}$ SB1518 is currently tested 
in a phase I/II trial. An other promising drug might be pomalidomide, a second-generation immunomodulatory drug. Pomalidomide was shown to improve anaemia (in 25\% of patients treated with $0.5 \mathrm{mg} /$ day and in $36 \%$ of patients treated with $3.0 \mathrm{mg} /$ dayl and platelet count in patients with $\leq 100 * 10^{9} / \mathrm{L}$ (in $58 \%$ patients treated with $0.5 \mathrm{mg} /$ day). ${ }^{[73,74]}$

Hypomethylating agents have also been investigated. The most promising is decitabine, which was tested in a phase II study in 21 MPN patients with myelofibrosis, showing a reduction of $61 \%$ in circulating $\mathrm{CD} 34^{+}$cells. ITF2357, a histone deacetylase inhibitor was shown to resolve pruritus in most patients, to reduce splenomegaly in $38 \%$ of the patients and showed a trend in reducing the JAK2 ${ }^{\mathrm{V} 617 F}$ allele burden. ${ }^{[75]}$

Everolimus (RAD001), inhibits the mammalian target of rapamycine (mTor) and was shown to reduce spleen size, to complete resolution of systemic symptoms and to reduce anaemia. Side effects were worsening of anaemia in $30 \%$ of the patients and grade two neutropenia or thrombocytopenia, although infrequent. ${ }^{[76]}$

The JAK inhibitors are the most promising new drug strategies for MPN patients with improvement in quality of life and relative minimal side effects. Although the long-term safety of these agents and if they prolong survival should be determined. Therefore JAK inhibitors should only be started as a form of therapy in myelofibrosis patients belonging to the intermediate-2 or high risk group. 


\section{REFERENCES}

1. Humphries, R.K., Eaves, A.C., Eaves, C.J., Self-renewal of hemopoietic stem cells during mixed colony formation in vitro. Proc Natl Acad Sci U S A, 1981. 78(6): p. 3629-33.

2. Giebel, B., Cell polarity and asymmetric cell division within human hematopoietic stem and progenitor cells. Cells Tissues Organs, 2008. 188(1-2): p. 116-26.

3. Akashi, K., Traver, D., Miyamoto, T., Weissman, I.L.., A clonogenic common myeloid progenitor that gives rise to all myeloid lineages. Nature, 2000. 404(6774): p. 193-7.

4. Akashi, K., Traver, D., Kondo, M., Weissman, I.L., Lymphoid development from hematopoietic stem cells. Int J Hematol, 1999. 69(4): p. 217-26.

5. Yoshihara, H., Arai, F., Hosokawa, K., et al., Thrombopoietin/MPL signaling regulates hematopoietic stem cell quiescence and interaction with the osteoblastic niche. Cell Stem Cell, 2007. 1(6): p. 685-97.

6. Orkin, S.H., Zon, L.I., Hematopoiesis: an evolving paradigm for stem cell biology. Cell, 2008. 132(4): p. 631-44.

7. Campbell, P.J., Green, A.R., The myeloproliferative disorders. N Engl J Med, 2006. 355(23): p. 2452-66.

8. Dameshek, W., Some speculations on the myeloproliferative syndromes. Blood, 1951. 6(4): p. 372-5.

9. Vardiman, J.W., Thiele, J., Arber, D.A., et al., The 2008 revision of the World Health Organization (WHO) classification of myeloid neoplasms and acute leukemia: rationale and important changes. Blood, 2009. 114(5): p. 937-51.

10. Budde, U., Scharf, R. E., Franke, P., Hartmann-Budde, K., Dent, J., Ruggeri, Z.M.,Elevated platelet count as a cause of abnormal von Willebrand factor multimer distribution in plasma. Blood, 1993. 82(6):

p. 1749-57.

11. Thiele, J., Kvasnicka, H.M., Fischer, R., Histochemistry and morphometry on bone marrow biopsies in chronic myeloproliferative disorders - aids to diagnosis and classification. Ann Hematol, 1999. 78(11): p. 495-506.

12. Murray, J., Myeloproliferative disorders. Clin Med, 2005. 5(4): p. 328-32.

13. Tefferi, A., How I treat myelofibrosis. Blood. 117(13): p. 3494-504.

14. Wadleigh, M., Tefferi, A., Classification and diagnosis of myeloproliferative neoplasms according to the 2008 World Health Organization criteria. Int J Hematol, 2010. 91(2): p. 174-9.

15. Vannucchi, A.M., Antonioli, E., Guglielmelli, P., Pardanani, A., Tefferi, A., Clinical correlates of JAK2 ${ }^{1617 F}$ presence or allele burden in myeloproliferative neoplasms: a critical reappraisal. Leukemia, 2008. 22(7): p. 1299-307.

16. Patel, R.K., Lea, N.C., Heneghan, M.A., et al., Prevalence of the activating JAK2 tyrosine kinase mutation V617F in the Budd-Chiari syndrome. Gastroenterology, 2006. 130(7): p. 2031-8.

17. Regina, S., Herault, O., D’Alteroche, L., Binet, C., Gruel, Y., JAK2 V617F is specifically associated with idiopathic splanchnic vein thrombosis. J Thromb Haemost, 2007. 5(4): p. 859-61.

18. Tefferi, A., Thiele, J., Orazi, A., et al., Proposals and rationale for revision of the World Health Organization diagnostic criteria for polycythemia vera, essential thrombocythemia, and primary myelofibrosis: recommendations from an ad hoc international expert panel. Blood, 2007. 110(4): p. 1092-7.

19. Tefferi, A., Vardiman, J.W., Classification and diagnosis of myeloproliferative neoplasms: the 2008 World Health Organization criteria and point-of-care diagnostic algorithms. Leukemia, 2008. 22(1): p. 14-22. 
20. Turkington, R.C., Arnold, E.C., Percy, M.J., Ranaghan, L.A., Cuthbert, R.J., McMullin, M.F., Comparison of diagnostic criteria for polycythaemia vera. Hematology, 2007. 12(2): p. 123-30.

21. Wilkins, B.S., Erber, W.N., Bareford, D. et al., Bone marrow pathology in essential thrombocythemia: interobserver reliability and utility for identifying disease subtypes. Blood, 2008. 111(1): p. 60-70.

22. Vardiman, J.W., Harris, N.L., Brunning, R.D., The World Health Organization (WHO) classification of the myeloid neoplasms. Blood, 2002. 100(7): p. 2292-302.

23. Vannucchi, A.M., Guglielmelli, P., Tefferi, A., Advances in understanding and management of myeloproliferative neoplasms. CA Cancer J Clin, 2009. 59(3): p. 171-91.

24. Baxter, E.J., Scott, L.M., Campbell, P.J., et al., Acquired mutation of the tyrosine kinase JAK2 in human myeloproliferative disorders. Lancet, 2005. 365(9464): p. 1054-61.

25. James, C., Ugo, V., Le Couedic, J.P., et al., A unique clonal JAK2 mutation leading to constitutive signalling causes polycythaemia vera. Nature, 2005. 434(7037): p. 1144-8.

26. Kralovics, R., Passamonti, F., Buser, A.S., et al., A gain-of-function mutation of JAK2 in myeloproliferative disorders. N Engl J Med, 2005. 352(17): p. 1779-90.

27. Levine, R.L., Wadleigh, M., Cools, J., et al., Activating mutation in the tyrosine kinase JAK2 in polycythemia vera, essential thrombocythemia, and myeloid metaplasia with myelofibrosis. Cancer Cell, 2005. 7(4): p. 387-97.

28. Jones, A.V., Kreil, S., Zoi, K., et al., Widespread occurrence of the JAK2 V617F mutation in chronic myeloproliferative disorders. Blood, 2005. 106(6): p. 2162-8.

29. Toyama, K., Karasawa, M., Yamane, A., et al., JAK2-V617F mutation analysis of granulocytes and platelets from patients with chronic myeloproliferative disorders: advantage of studying platelets. $\mathrm{Br} J$ Haematol, 2007. 139(1): p. 64-9.

30. Florensa, L., Bellosillo, B., Besses, C., et al., JAK2 V617F mutation analysis in different myeloid lineages (granulocytes, platelets, CFU-MK, BFU-E, and CFU-GM) in essential thrombocythemia patients. Leukemia, 2006. 20(10): p/1903-5.

31. Tiedt, R., Hao-Shen, H., Sobas, M.A., et al., Ratio of mutant JAK2-V617F to wild-type JAK2 determines the MPD phenotypes in transgenic mice. Blood, 2008. 111(8): p. 3931-40.

32. Marchioli, R., Finazzi, G., Landolfi, R., et al., Vascular and neoplastic risk in a large cohort of patients with polycythemia vera. J Clin Oncol, 2005. 23(10): p. 2224-32.

33. Berk, P.D., Goldberg, J.D., Donovan, P.B., Fruchtman, S.M., Berlin, N.I., Wasserman, L.R., Therapeutic recommendations in polycythemia vera based on Polycythemia Vera Study Group protocols. Semin Hematol, 1986. 23(2): p. 132-43.

34. Finazzi, G., Barbui, T., Risk-adapted therapy in essential thrombocythemia and polycythemia vera. Blood Rev, 2005. 19(5): p. 243-52.

35. Finazzi, G., Barbui, T., Evidence and expertise in the management of polycythemia vera and essential thrombocythemia. Leukemia, 2008. 22(8): p. 1494-502.

36. Harrison, C.N., Campbell, P.J., Buck, G., et al., Hydroxyurea compared with anagrelide in high-risk essential thrombocythemia. N Engl J Med, 2005. 353(1): p. 33-45.

37. Tartaglia, A.P., Goldberg, J.D., Berk, P.D., Wasserman, L.R., Adverse effects of antiaggregating platelet therapy in the treatment of polycythemia vera. Semin Hematol, 1986. 23(3): p. 172-6.

38. Gruppo Italiano Studio Policitemia (GISP)., Low-dose aspirin in polycythaemia vera: a pilot study. Br J Haematol, 1997. 97(2): p. 453-6. 
39. Landolfi, R., Marchioli, R., Kutti, J., et al., Efficacy and safety of low-dose aspirin in polycythemia vera. N Engl J Med, 2004. 350(2): p. 114-24.

40. Scott, R.B., Treatment of polycythaemia rubra vera. Br Med J, 1953. 1(4820): p. 1128-31.

41. Fruchtman, S.M., Mack, K.,Kaplan, M.E., Peterson, P., Berk, P.D., Wasserman, L.R., From efficacy to safety: a Polycythemia Vera Study group report on hydroxyurea in patients with polycythemia vera. Semin Hematol, 1997. 34(1): p. 17-23.

42. Silver, R.T., Recombinant interferon-alpha for treatment of polycythaemia vera. Lancet, 1988. 2(8607): p. 403.

43. Silver, R.T., Long-term effects of the treatment of polycythemia vera with recombinant interferonalpha. Cancer, 2006. 107(3): p. 451-8.

44. Jabbour, E., Kantarjian, H., Cortes, J., et al., PEG-IFN-alpha-2b therapy in BCR-ABL-negative myeloproliferative disorders: final result of a phase 2 study. Cancer, 2007. 110(9): p. 2012-8.

45. Samuelsson, J., Hasselbalch, H., Bruserud, 0., et al., A phase II trial of pegylated interferon alpha-2b therapy for polycythemia vera and essential thrombocythemia: feasibility, clinical and biologic effects, and impact on quality of life. Cancer, 2006. 106(11): p. 2397-405.

46. Quintas-Cardama, A., Kantarjian, H., Manshouri, T., et al., Pegylated interferon alfa-2a yields high rates of hematologic and molecular response in patients with advanced essential thrombocythemia and polycythemia vera. J Clin Oncol, 2009. 27(32): p. 5418-24.

47. Kiladjian, J.J., Cassinat, B., Chevret, S., et al., Pegylated interferon-alfa-2a induces complete hematologic and molecular responses with low toxicity in polycythemia vera. Blood, 2008. 112(8): p. 3065-72.

48. Landolfi, R., Nicolazzi, M.A., Porfidia, A., Di Gennaro, L., Polycythemia vera. Intern Emerg Med, 2010. 5(5): p.411-3.

49. Cervantes, F., Dupriez, B., Pereira, A., et al., New prognostic scoring system for primary myelofibrosis based on a study of the International Working Group for Myelofibrosis Research and Treatment. Blood, 2009. 113(13): p. 2895-901.

50. Passamonti, F., Cervantes, F., Vannucchi, A.M., et al., A dynamic prognostic model to predict survival in primary myelofibrosis: a study by the IWG-MRT (International Working Group for Myeloproliferative Neoplasms Research and Treatment). Blood, 2010. 115(9): p. 1703-8.

51. Gangat, N., Caramazza, D., Vaidya, R., et al., DIPSS plus: a refined Dynamic International Prognostic Scoring System for primary myelofibrosis that incorporates prognostic information from karyotype, platelet count, and transfusion status. J Clin Oncol, 2011. 29(4): p. 392-7.

52. Barbui, T., Barosi, G., Birgegard, G., et al., Philadelphia-negative classical myeloproliferative neoplasms: critical concepts and management recommendations from European LeukemiaNet. J Clin Oncol, 2011. 29(6): p. 761-70.

53. Mishchenko, E., Tefferi, A., Treatment options for hydroxyurea-refractory disease complications in myeloproliferative neoplasms: JAK2 inhibitors, radiotherapy, splenectomy and transjugular intrahepatic portosystemic shunt. Eur J Haematol, 2010. 85(3): p. 192-9.

54. Holle, N., de Witte, T., Mandigers, C., Schaap, N., Raymakers, R., Thalidomide and lenalidomide in primary myelofibrosis. Neth J Med, 2010. 68(1): p. 293-8.

55. Tefferi, A., Vainchenker, W., Myeloproliferative Neoplasms: Molecular Pathophysiology, Essential Clinical Understanding, and Treatment Strategies. J Clin Oncol, 2011. 29(5): p. 573-82. 
56. Cervantes, F., Modern management of myelofibrosis. Br J Haematol, 2005. 128(5): p. 583-92.

57. Cervantes, F., Alvarez-Larran, A., Domingo, A., Arellano-Rodrigo, E., Montserrat, E., Efficacy and tolerability of danazol as a treatment for the anaemia of myelofibrosis with myeloid metaplasia: longterm results in 30 patients. Br J Haematol, 2005. 129(6): p. 771-5.

58. Cervantes, F., Alvarez-Larran, A., Hernandez-Boluda, J.C., Sureda, A., Torrebadell, M., Montserrat, E., Erythropoietin treatment of the anaemia of myelofibrosis with myeloid metaplasia: results in 20 patients and review of the literature. Br J Haematol, 2004. 127(4): p. 399-403.

59. Cervantes, F., Hernandez-Boluda, J.C., Alvarez, A., Nadal, E., Montserrat, E., Danazol treatment of idiopathic myelofibrosis with severe anemia. Haematologica, 2000. 85(6): p. 595-9.

60. Cervantes, F., Mesa, R., Barosi, G., New and old treatment modalities in primary myelofibrosis. Cancer J, 2007. 13(6): p. 377-83.

61. Stewart, W.A., Pearce, R., Kirkland, K. E., et al., The role of allogeneic SCT in primary myelofibrosis: a British Society for Blood and Marrow Transplantation study. Bone Marrow Transplant, 2010. 45(11): p. 1587-93.

62. Robin, M., Tabrizi, R., Mohty, M., et al., Allogeneic haematopoietic stem cell transplantation for myelofibrosis: a report of the Societe Francaise de Greffe de Moelle et de Therapie Cellulaire (SFGMTC). Br J Haematol, 2011. 152(3): p. 331-9.

63. Lissandre, S., Bay, J.O., Cahn, J.Y., et al., Retrospective study of allogeneic haematopoietic stem-cell transplantation for myelofibrosis. Bone Marrow Transplant, 2011. 46(4): p. 557-61.

64. Kroger, N., Holler, E., Kobbe, G., et al., Allogeneic stem cell transplantation after reduced-intensity conditioning in patients with myelofibrosis: a prospective, multicenter study of the Chronic Leukemia Working Party of the European Group for Blood and Marrow Transplantation. Blood, 2009. 114(26): p. 5264-70.

65. Gaikwad, A., Verstovsek, S., Yoon, D., et al., Imatinib effect on growth and signal transduction in polycythemia vera. Exp Hematol, 2007.35(6): p. 931-8.

66. Verstovsek, S., et al., Safety and Efficacy of INCB018424, a JAK1 and JAK2 Inhibitor, in Myelofibrosis. N Engl J Med. 363(12): p. 1117-27.

67. Verstovsek, S., Kantarjian, H., Mesa, R.A., et al., Results of COMFORT-I, a Randomized Double-Blind, Phase III Trial of the JAK1 and JAK2 Inhibitor Ruxolitinib (INCB18424) versus Placebo for Patients With Myelofibrosis. Oral Communication, EHA, 2011.

68. Tefferi, A., Pardanani, A., Serious Adverse Events During Ruxolitinib Treatment Discontinuation in Patients With Myelofibrosis. Mayo Clin Proc, 2011. 86(12): p. 1188-91.

69. Pardanani, A., Gotlib, J.R., Jamieson, C., et al., Safety and efficacy of TG101348, a selective JAK2 inhibitor, in myelofibrosis. J Clin Oncol, 2011. 29(7): p. 789-96.

70. Pardanani, A., George, G., Lasho, T., et al., A Phase I/II Study of CYT387, An Oral JAK-1/2 Inhibitor, In Myelofibrosis: Significant Response Rates In Anemia, Splenomegaly, and Constitutional Symptoms. Blood, 2010. 117 Abstr. 460.

71. Santos, F.P., Kantarjian, H.M., Jain, N., et al., Phase 2 study of CEP-701, an orally available JAK2 inhibitor, in patients with primary or post-polycythemia vera/essential thrombocythemia myelofibrosis. Blood, 2010. 115(6): p. 1131-6. 
72. Verstovsek, S., Odenike, O., Scott, B., et al., Phase I Dose-Escalation Trial of SB1518, a Novel JAK2/FLT3 Inhibitor, in Acute and Chronic Myeloid Diseases, Including Primary or Post-Essential Thrombocythemia/ Polycythemia Vera Myelofibrosis. Blood, 2009. 114 Abstr. 3905.

73. Begna, K.H., Mesa, R.A., Pardanani, A., et al., A phase-2 trial of low-dose pomalidomide in myelofibrosis. Leukemia, 2010. 25(2): p. 301-4.

74. Mesa, R.A., Pardanani, A.D., Hussein, K., et al., Phase1/-2 study of Pomalidomide in myelofibrosis. Am J Hematol, 2010. 85(2): p. 129-30.

75. Rambaldi, A., Dellacasa, C.M., Finazzi, G., et al., A pilot study of the Histone-Deacetylase inhibitor Givinostat in patients with JAK2 $2^{\mathrm{V} 617 \mathrm{~F}}$ positive chronic myeloproliferative neoplasms. Br J Haematol, 2010. 150(4): p. 446-55.

76. Vannucchi, A.M., Guglielmelli, P., Lupo, L., et al., A Phase 1/2 Study of RAD001, a mTOR Inhibitor, In Patients with Myelofibrosis: Final Results. Blood, 2010. 116:314 (ASH Annual Meeting Abstracts). 


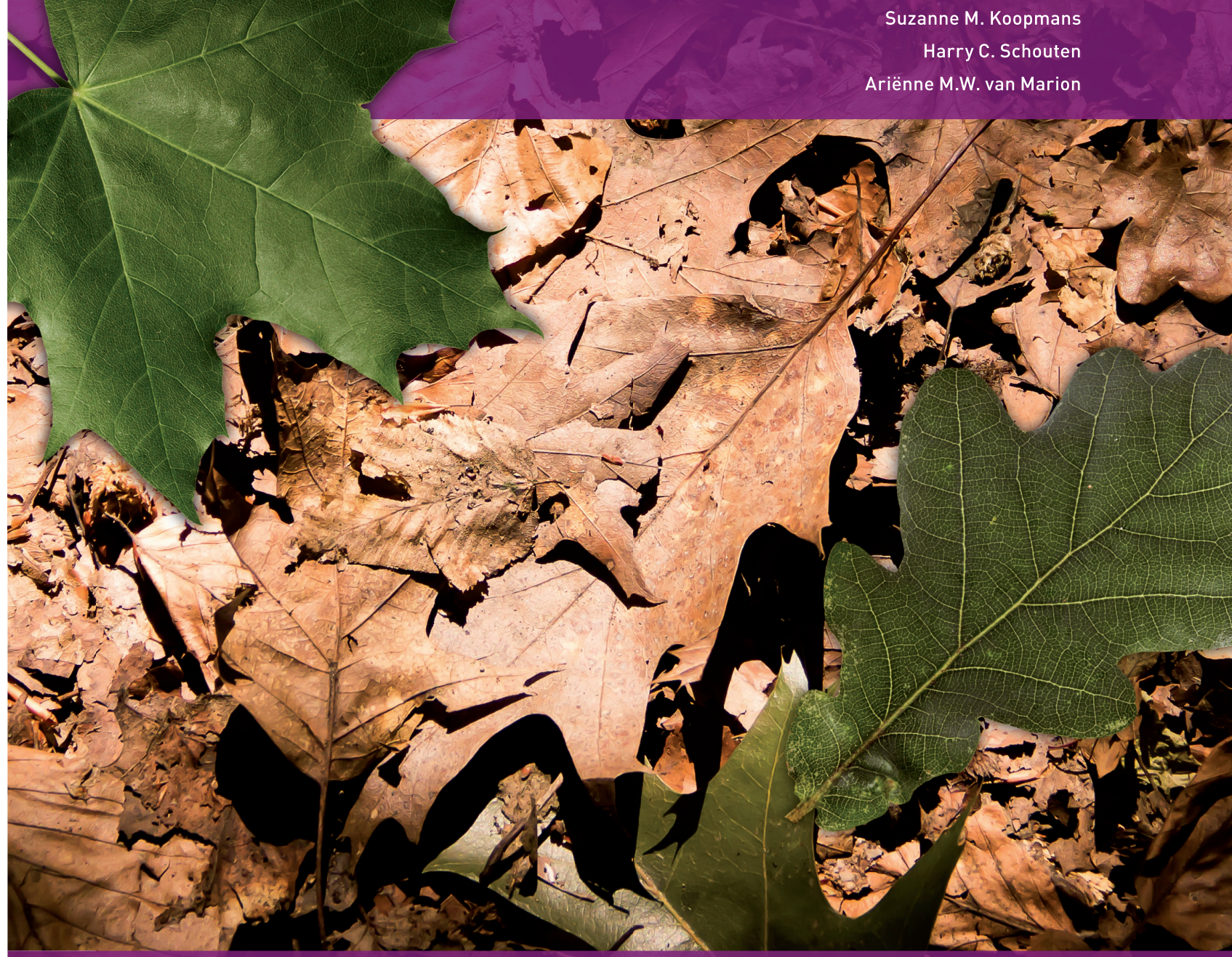

Accepted in Histology and Histopathology 


\section{ABSTRACT}

The clonal bone marrow stem cell disorders essential thrombocythemia (ET), polycythemia vera (PV) and primary myelofibrosis (PMF) belong to the group of Philadelphia chromosome negative myeloproliferative neoplasia (Ph- MPN). In 2005 the JAK2 ${ }^{\text {Vil7F }}$ mutation was discovered which generated more insight in the pathogenetic working mechanism of the MPNs. Since then, more mutations have been detected in MPN patients. However, the underlying cause of MPN has not been discovered so far. The mechanism of increased angiogenesis in MPNs and the development of fibrosis in the bone marrow in PMF patients and in some ET and PV patients is still not known. This review will focus on the most important molecular pathogenetic mechanisms of MPN patients. 


\section{INTRODUCTION}

Luis Henry Vaquez together with William Osler described in 1892 for the first time a case report of "Osler-Vaquez disease", nowadays known as polycythemiavera. ${ }^{[1]}$ In 1951 William Dameshek introduced the term "myeloproliferative disorders" (MPDs) to describe five different diseases with similarities in clinical phenotype and the belief of an underlying undiscovered stimulus responsible for the proliferative activity of bone marrow cells in these diseases. ${ }^{[2]}$ Since then, more knowledge of the MPDs has been generated; from the discovery of the Philadelphia (Ph) chromosome in chronic myeloid leukaemia (CML) via the demonstration of clonality in MPDs to the discovery of the JAK2 ${ }^{\text {V617F }}$ mutation in MPDs. ${ }^{[3-11]}$ Later on, the World Health Organization (WHO) recommended changing the term myeloproliferative disorders to myeloproliferative neoplasia (MPN) to underline the clonality of the diseases. According to the 2008 WHO classification the MPNs include essential thrombocythemia (ET), polycythemia vera (PV), primary myelofibrosis (PMF), CML, chronic neutrophilic leukaemia (CNL), chronic erythroleukemia (CEL), systemic mastocytosis (SM) and MPN unclassifiable. The classical MPNs include ET, PV, PMF and CML. ${ }^{[12]}$

Nowadays, the classicalPhiladelphia chromosome negative (Ph-) MPNsincludeET, PVand PMF. ${ }^{[13,14]}$ The proliferation of one or more of the erythroid, myeloid and megakaryocytic cell lineages characterizes the MPNs. Proliferation of the megakaryocytic cell line in ET patients results in increased blood platelets in the peripheral blood. PV is characterized by a trilineage proliferation resulting in mainly increased erythrocytes but often also leukocytes and blood platelets are increased in the peripheral blood. The bone marrow of PMF patients is characterized by a proliferation of the megakaryocytic and granulocytic cell line and the development of fibrosis. ${ }^{[14,15]}$ In this review we will focus on the molecular pathogenetic mechanisms involved in the MPNs. First, we will focus on the JAK-STAT pathway followed by the JAK2 ${ }^{\mathrm{V} 617 F}$ mutation. Then the review will continue with the influence of the JAK2 $2^{\text {V617F }}$ mutation on the JAK-STAT, PI3K-Akt and Erk pathway in MPN patients. Other recently discovered mutations will then be discussed in JAK2 negative MPN patients. Finally, the pathogenesis of angiogenesis and myelofibrosis will be discussed. 


\section{THE JAK-STAT PATHWAY}

The presence of a JAK2 mutation is an important diagnostic clue to the diagnosis of Ph- MPN. The family of Janus Kinases (JAKs) consists of four members: JAK1, JAK2, JAK3 and Tyk2. The binding of cytokines, growth factors or hormones on cell surface receptors of the JAK family leads to transphosphorylation between the associated JAKs, resulting in tyrosine phosphorylation of the receptors and synthesis of binding sites for downstream signalling proteins. The signal transducer and activators of transcription (STATs) remain unphosphorylated in the cytoplasm of a quiescent cell until they become activated after tyrosine phosphorylation. The STATs are recruited to these binding sites, become phosphorylated, dimerize and translocate to the nucleus where they stimulate transcription (see Figure 1)..$^{[16-18]}$

Figure 1: Positive and negative regulation of the JAK-STAT signal transduction pathway.

Binding of cytokines, growth factors or hormones on cell surface receptor transphosphorylates associated JAKs (Janus Kinase) creating binding sites for downstream signalling proteins. STATs (signal transducer and activator of transcriptions) become activated upon tyrosine phosphorylation, are recruited to the binding sites and become phosphorylated resulting in stimulation of transcription. The SOCS (suppressors of cytokine signalling), activated by STATs, provide a negative feedback loop to JAKs and STATs. PTPS (phosphotyrosine phosphatases) and PIASs (protein inhibitors of activated STATs) inhibit JAKs, STATs or cytokine receptors.

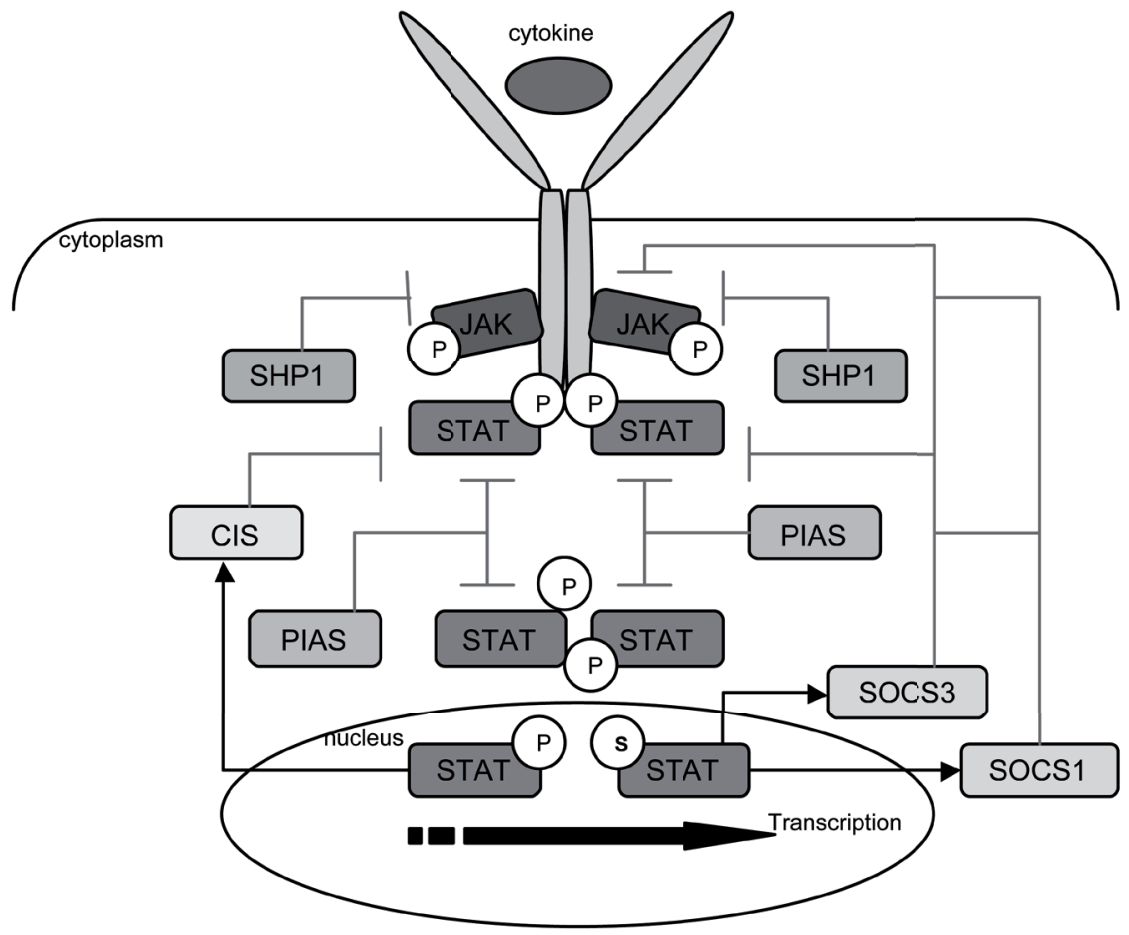


The STAT family consists of seven STATs: STAT1, STAT2, STAT3, STAT4, STAT5a, STAT5b and STAT6. ${ }^{[18]}$

The JAK/STAT pathway is inhibited by three protein families (see Figure 1):

1) Haematopoietic cells express SHP1.SHP1 belongs to the family of phosphotyrosine phosphatases (PTP); PTP dephosphorylates activated JAKs, STATs or cytokine receptors. ${ }^{[19,20]}$

2) Suppressors of cytokine signalling (SOCS) interact with activated JAKs or phosphorylated receptors or they induce the JAK proteasomal degradation. CIS, SOCS1, SOCS2 and SOCS3 are members of the SOCS protein family. The synthesis of SOCS is induced by activated STATs resulting in a negative feedback loop, through interacting with activated JAKs resulting in inhibition of STAT recruitment to the binding sites. ${ }^{[16,21]}$

3) Protein inhibitors of activated STATs (PIAS), they interact with activated STATs and inhibit their dimerisation. ${ }^{[19]}$

\section{The JAK2 $2^{\mathrm{V} 617 \mathrm{~F}}$ mutation}

Until 2005 little was known about the aetiology of MPN. The discovery of the JAK2 $2^{\text {vil7F }}$ mutation in MPN patients ${ }^{[3-6]}$ gave a better understanding of the pathogenesis of MPNs. The JAK2 ${ }^{\mathrm{V} 617 F}$ mutation is present in granulocytes, erythroblasts and myeloblasts and in all erythropoietine (EPO)-independent erythroid colonies. The JAK2 ${ }^{\text {V617F }}$ mutation deregulates the JAK2 kinase activity by destabilizing the inhibitory interaction, resulting in increased JAK2 kinase activity. Altogether, this results in a sustained JAK2 activation, while the feedback mechanism has been destroyed. ${ }^{[3]}$

The erythroid colonies with the JAK2 ${ }^{\mathrm{V} 617 F}$ mutation are able to grow in the absence of EPO. Therefore, the JAK2 $2^{\mathrm{V} 17 F}$ mutation results in factor independent growth of the erythroid cell line. ${ }^{[22]}$ The receptors of bone marrow progenitor cells are hypersensitive to thrombopoietine (TPO, stimulates proliferation and differentiation of megakaryocytes), EPO (stimulates erythroblasts), stem cell factor (SCF, induces proliferation and selfrenewal of multipotent haematopoietic progenitors) and granulocyte stimulating factor (GSF, stimulates proliferation and differentiation of granulocytes). The hypersensitivity of these cytokines results in monoclonal stimulation of the megakaryopoiesis, erythropoiesis and granulopoiesis. However, due to JAK2 V617F and other mutations, haematopoietic progenitor cells can proliferate without the presence or induction by cytokines. ${ }^{[23]}$

It is unclear how one mutation can be responsible for three phenotypically different diseases. Besides, not every MPN patient carries the JAK2 $2^{\text {vi7F }}$ mutation; $>95 \%$ of the 
PV patients and about $50 \%$ of the ET and PMF patients carry the JAK2 $2^{\text {V617F }}$ mutation. This generated three hypothesis. The first hypothesis is the 'gene-dosage' hypothesis, which postulates a correlation between phenotype and allele burden, which is the ratio between mutant and wild type JAK2 in haematopoietic cells; between MPN patients there is a variability in the number of cells carrying the mutation and there is a variability in the alleles that carry the JAK2 $2^{\text {V617F }}$ mutation. Most PV patients are homozygous for the JAK2 $2^{\text {V617F }}$ mutation, while ET patients are heterozygous. Indeed, some ET patients with increasing allele burden transform over time to PV or PMF. Importantly, ET patients with the JAK2 $2^{V 617 F}$ mutation have a "PV-like" phenotype compared to ET patients whithout the mutation. Patients with a MPN carrying the JAK2 ${ }^{\text {V617F }}$ mutation do not have a higher risk of developing post-PV and post-ET myelofibrosis compared to patients without the JAK2 mutation ${ }^{[24-27]}$. Controversy exists of the allele burden and the risk of developing acute myeloid leukaemia (AML). ${ }^{[28,29]}$ It was shown that some patients with a JAK2 ${ }^{\text {V617F }}$ positive MPN can lose this JAK2 mutation in the progression to AML. A possible explanation for this phenomenon is the concept of a 'pre-JAK2' phase in which other mutations are responsible for the progression to AML. This 'pre-JAK2 phase' is a second hypothesis which postulates additional somatic mutations or inherited predisposing alleles present before the JAK2 $2^{\mathrm{V} 617 F}$ mutation, which are responsible for the clonal haematopoiesis and determine the phenotype. This pre-JAK2 phase might even be responsible for generating the JAK2 ${ }^{\text {V617F }}$ mutation or they might act synergistically. ${ }^{[18,19]}$ The 'gene-dosage' and preJAK2 phase' do not entirely explain the phenotypic heterogeneity of the MPNs; differences in mice with different genetic backgrounds were observed leading to a third hypothesis of 'host genetic factors'. This hypothesis postulates that host genetic characteristics might act as modifiers of the phenotype in combination with the JAK2 ${ }^{\mathrm{V} 617 \mathrm{~F}}$ mutation, for instance, singe nucleotide polymorphisms (SNPs), even gender is an independent modifier with women having a lower JAK2 ${ }^{\mathrm{V} 617 \mathrm{~F}}$ allele burden than men. ${ }^{[30-32]}$

Also, the coexistence of independent JAK2-mutant and JAK2 wild-type clonal expansions in the same patient can be an explanation. It is observed that JAK2 positive AML patients are preceded by transformation to myelofibrosis during their disease course, in contrast to. JAK2 wild-type AML which is preceded by chronic-phase ET and PV patients. The mechanism of this disease evolution remains to be elucidated. ${ }^{[33]}$

Recent studies with JAK(1//2 inhibitors have shown that the symptoms of MPN patients are dramatically improved in patients with the JAK2 ${ }^{\text {V617F }}$ mutation but also in patients with wild type JAK2 gene. Ruxolitinib (INCB018424), SAR302503 (TG101348) and CYT387 are promising JAK(1//2 inhibitors when evaluating the improvement of the symptoms and the side effects. The side effects of JAK inhibitors include thrombocytopenia, anaemia and neutropenia and gastro-intestinal symptoms like diarrhoea, nausea and 
vomiting. Further, with ruxolitinib a withdrawal syndrome has been reported during discontinuation of ruxolitinib characterized by acute relapse of disease symptoms, accelerated splenomegaly and worsening of cytopenias. ${ }^{[34-39]}$ Although the JAK2 ${ }^{\text {V617F }}$ mutation is an important underlying mechanism, it is not the cause of the MPN. Therefore, when using the JAK inhibitors a balance should be found for each individual patient with enough benefit and the least side effects. The fact that patients with wild type JAK2 also benefit from JAK(1/)2 inhibitors indicates other, still unknown, underlying mechanism(s) responsible for the increased JAK/STAT pathway activity in MPN patients.

\section{The JAK-STAT, PI3K-Akt and Erk pathway and MPN}

The sustained activation of JAK2 increases STAT3 and STAT5 phosphorylation. Activated STAT3 regulates cell growth through regulation of cyclin D1 and induces Bcl-2 Isee Figure 2) resulting in an anti-apoptotic signal. STAT3 was also shown to play an important role in megakaryopoiesis, mainly through the expansion of megakaryocytic progenitor cells. ${ }^{[40-42]}$ Activated STAT5 up-regulates $\mathrm{Bcl}-\mathrm{x}_{\mathrm{L}}$ (see Figure 2), inhibiting apoptosis of megakaryocytes, and mediates cell growth through induction of cyclin D1. ${ }^{[43,44]}$ The net result of STAT3 and STAT5 activation is apoptosis inhibition and a proliferative activity.

Due to the JAK2 ${ }^{\text {V617F }}$ mutation the sustained JAK2 activation suggests an up-regulation in phosphorylated STAT3 (pSTAT3) and STAT5 (pSTAT5) in MPN patients, probably especially in JAK2 positive patients, while STAT3 and STAT5 are activated by JAK2. Indeed, pSTAT5 was shown to be up-regulated in JAK2 ${ }^{\text {V617F }}$ positive MPN patients. ${ }^{[45]}$

The JAK2 ${ }^{\text {V617F }}$ mutation also activates the MAPK and PI3K-Akt pathway (see Figure 3). ${ }^{[4,6]}$ The JAK2 ${ }^{\mathrm{V} 617 \mathrm{~F}}$ mutation and PSTAT5 can both activate PI3K by interacting with $\mathrm{p} 85$, a regulatory subunit of PI3K. Activated PI3K activates Akt, which in turn activates mTor on Ser2448 which directly phosphorylates ribosomal p70S6Kinase (p70S6k). ${ }^{[46,47]}$ The phosphatidylinositol-3-kinase (PI3K)/Akt/mammalian target of rapamycine (mTor) pathway is known to be commonly activated in leukaemia and lymphoma and is known to play a role in inhibiting apoptosis in normal human erythroblasts. ${ }^{[48,49]}$ The PI3K/ Akt pathway induces the phosphorylation of BAD, a pro-apoptotic member of the Bcl-2 family, via phosphorylated Akt (pAkt) and p70S6k. The BAD function is inhibited upon BAD phosphorylation resulting in inhibition of apoptosis. Activation of the PI3K/Akt pathway also induces the activation of $\mathrm{BCl}-\mathrm{x}_{\mathrm{L}}$, resulting in inhibition of megakaryocyte apoptosis (see Figure 2). ${ }^{[50-52]}$

Due to the inactivation of the pro-apoptotic factor BAD and activation of $\mathrm{Bcl}-\mathrm{xL}, \mathrm{Akt}$ suppresses apoptosis and promotes cell survival. ${ }^{[53-57]}$ Since the bone marrow of MPN patients is characterised by a pathological increase in megakaryocytes, Akt might play an important role in the up-regulation of megakaryocytes, possibly secondary to the 
Figure 2: Signalling pathway of JAK-STAT, Erk and PI3K/Akt.

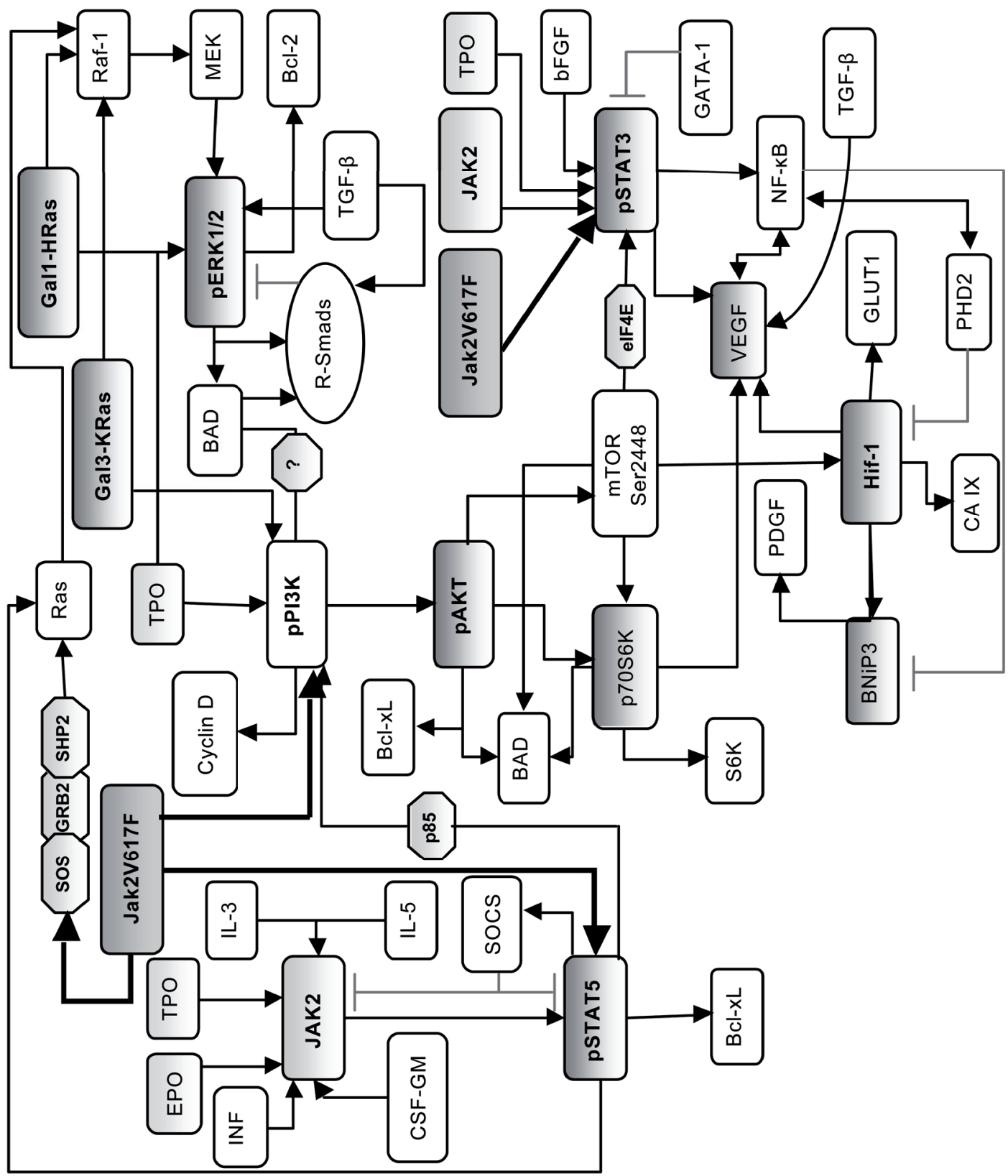


Figure 2: Signalling pathway of JAK-STAT, Erk and PI3K/Akt.

JAK2 (Janus Kinase) is activated by different cytokines, growth factors and hormones (erythropoetine (EPO), thrombopoetine (TPO), interleukin (IL)-3 and -5, stem colony stimulating factor-granulocytemacrophage (CSF-GM) and interferon (INF)). JAK2 phosphorylates STAT5 (signal transducer and activator of transcription) and STAT3, however the JAK2 ${ }^{\mathrm{V} 617 F}$ mutation induces a sustained activation of STAT5, STAT3, $\mathrm{PI} 3 \mathrm{~K}$ (phosphatidylinostiol-3 kinase) and indirect of extracellular signal-regulated kinase (Erk) resulting in increased activation of their downstream proteins. Phosphorylated STAT5 (pSTAT5) and pSTAT3 induces $\mathrm{Bcl}-\mathrm{xL}$ resulting in megakaryocyte apoptosis. STAT3 is not only activated by JAK2 and the JAK2 ${ }^{\mathrm{V} 617 F}$ mutation but also by TPO and fibroblast growth factor (FGF). Phosphorylated PI3K (pPI3K) phosphorylates AKT (pAKT) activating $\mathrm{Bcl}-\mathrm{xL}$ and inhibits $\mathrm{BAD}$ resulting in inhibition of apoptosis. pAKT also activates the mammalian target of rapamycin complex 1 (mTORC1) which can also phosphorylate STAT3 and activates ribosomal

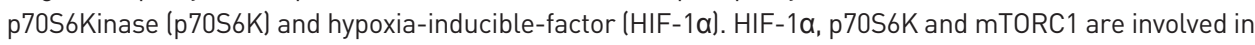
angiogenesis by activation of vascular endothelial growth factor (VEGF). Phosphorylated ERK (pERK) is not only indirectly activated by the JAK2 $2^{\text {V617F }}$ mutation but also directly by TPO, galectin-1 (gal-1) and gal-3. pERK activates $\mathrm{Bcl}-2$ and $\mathrm{BAD}$ with a net result of inhibiting apoptosis.

$J A K 2^{\text {V617F }}$ mutation. It was already demonstrated in previous studies that an increased phosphorylation of Akt is present in JAK2 $2^{\mathrm{V} 617 F}$ positive patients Is also associated with increased erythropoiesis in PV patients. ${ }^{[45,58,59]}$

An increased activation of Ras-Erk signalling pathway has been demonstrated in PV patients. ${ }^{[58]}$ Extracellular signal-regulated kinase (Erk) belongs to the mammalian MAPK families which are serine-threonine kinases. ${ }^{[60]}$ This receptor tyrosine kinase (RTK)-Grb2-SOS signalling axis activates GTPase Ras which in turn activates Raf-1. Raf-1 mediates the activation of MEK which in turn activates Erk (see Figure 2). ${ }^{[61]}$ Erk phosphorylates BAD, resulting in inactivation of BAD with inhibition of apoptosis, and activation of $\mathrm{Bcl}-2$ to form homodimers generating anti-apoptotic responses. ${ }^{\left[{ }^{[62]}\right.}$ The net result of Erk phosphorylation is the inhibition of apoptosis, by blocking the function of BAD and activation of Bcl-2. Therefore, the activation of the Erk pathway is suggested be one of the mechanisms responsible for the hypercellularity seen in the bone marrow of MPN patients, also possibly secondary to the JAK2 ${ }^{\mathrm{V} 617 F}$ mutation.

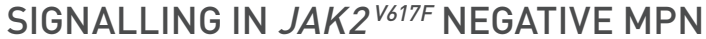

Before the discovery of the JAK2 ${ }^{\mathrm{V} 617 F}$ mutation, the molecular pathogenesis of the MPN was unknown. It was thought that ET, PV and PMF were clonal disorders arising in a multipotent progenitor cell characterized by independency or hypersensitivity of haematopoietic progenitors to numerous cytokines. The PV progenitors are hypersensitive to EPO, leading to the forming of erythroid progenitor cells in the absence of EPO. In PMF there is an enhanced sensitivity to TPO, with a defective TPO-receptor in 
which the negative regulation is disturbed, and this is also seen in PV. Recently, another mutation in the JAK2 gene was found, the JAK2 exon 12 mutation, only found in JAK2 $2^{\text {617F }}$ negative PV patients and contributes primarily to erythroid myeloproliferation. This mutation in JAK2 exon 12 leads to increased levels of phosphorylated JAK2, STAT5 and Erk compared to patients with wild type JAK2 and even higher phosphorylated JAK2 and Erk levels compared to patients with the JAK2 $2^{\mathrm{V} 617 F}$ mutation. PV patients with the JAK2 exon 12 mutation are of younger age and have normal leukocyte and platelet counts compared to PV patients with the JAK2 ${ }^{\mathrm{V} 617 F}$ mutation. ${ }^{[63,64]}$

Another additional mutation is present in the myeloproliferative leukaemia oncogene (MPL), which encodes for the TPO-receptor, present in 5\% of JAK2 $2^{\mathrm{V} 617 F}$ negative ET patients and in $10 \%$ of $J A K 2^{V 617 F}$ negative PMF patients. This gain of function mutation also results in activation of JAK2, STAT3, STAT5, Erk and Akt. ${ }^{[65,66]}$ ET patients with an MPL mutation are of older age, have lower haemoglobin levels and higher platelet counts. MPL-mutated PMF patients are also of older age with lower haemoglobin levels. ${ }^{[65,67]}$ Therefore, these mutations seem to have different phenotypes compared to MPN JAK2 ${ }^{\text {V617F }}$ positive MPN patients.

Other mutations are found in MPN patients (see Table 1), involving LNK mutations. Wildtype $L N K$ inhibits the constitutive activity of $J A K 2^{V 617 F}$ and $M P L$, therefore mutations in $L N K$ result in neutralising this inhibition. However $L N K$ mutations are also found in other myeloid malignancies and therefore are not specific for MPN. LNK mutations include TenEleven-Translocation2 (TET2) mutations important in DNA demethylation, Additional Sex Comb-Like1 (ASXL 1) mutations, Isocitrate Dehydrogenase 1/2 (IDH1/IDH2) mutations and Enhancer of Zeste Homolog 2 (EZH2) mutations. LNK negatively regulates the JAK2 activation via the TPO-receptor and is shown to be mutated in all MPN at a low frequency in patients with the JAK2 ${ }^{\mathrm{V} 617 F}$, TET2, IDH and/or MPL mutations, but was found more often in leukemic transformation of MPN (13\%). ${ }^{[68-73]}$

Epigenetic mechanisms can be modulated via regulation of transcription involving DNA and histone modifications. The TET2, ASXL1, IDH1/2 and DNA cytosine methyltransferase 3a (DNMT3A) belong to these epigenetic modifiers. Mutations in these epigenetic modifiers are predominantly found in PMF patients and blast phase MPN patients. ASXL1, IDH1/2, DNMT3A and EZH2 mutations are associated with poor survival in MPN patients.

The discovery of so many mutations in MPN patients suggests that they are primarily background mutations and several affect the same pathways. Some mutations are involved in early phase MPN (ASXL1), some in chronic phase MPN (JAK2 ${ }^{\text {V617F }, ~ M P L) ~ a n d ~ s o m e ~ i n ~}$ disease progression (LNK, TET2, IDH1/2 and DNMT3A). By a higher frequency of some mutations in myelofibrosis patients (PMF, post-ET/PV myelofibrosis) they might predict disease progression to myelofbrosis in PV and ET patients (ASXL1). Other mutations are found in higher frequency in AML patients preceded by a MPN (LNK, DNMT3A). ${ }^{[73,74]}$ 
Except for the known mutations, bone marrow cells, like megakaryocytes, stem cells, progenitor cells and myeloid cells, can produce aberrant cytokines, activating signalling in MPNs. These cytokines are produced in mutant and non-mutant MPN cells. In other diseases the STAT3 and NF-K $\beta$ signalling have been found to regulate cytokine transcription. ${ }^{[75]}$ Treatment with JAK inhibitors and also STAT3 deletion can both reduce cytokine expression in MPN cells, giving us the reason why JAK negative MPN patients can still respond clinically on JAK inhibitory therapy. ${ }^{[76]}$ The complex of the different molecular aberrations together with the production of aberrant cytokine signalling is believed to be the pathogenic mechanism initiating MPN disease.

\section{ANGIOGENESIS, MYELOFIBROSIS AND MPN}

Angiogenesis is the formation of new blood vessels and capillaries from existing blood vessels, playing an important role in the pathogenesis of many human malignancies and non-malignant diseases. By several studies it was shown that the micro vessel density (MVD) is increased in the bone marrow of MPN patients, with the highest MVD in PMF patients. The MVD was also found to be higher in MPN patients with the JAK2 mutation compared to MPN patients who do not carry the JAK2 mutation. ${ }^{[63-67]}$

Angiogenesis is tightly regulated by pro-and anti-angiogenic factors. ${ }^{[68]}$ Hypoxia-inducible-factor-1 (Hif-1) is a major regulator of angiogenesis. Hif-1 is activated under hypoxic conditions. Hif-1 consists of Hif- $1 \alpha$, expressed under hypoxic conditions, and Hif-1 $\beta$, expressed under normoxic and hypoxic conditions. The Hif-1 protein becomes active only when the $\alpha$ - and $\beta$-subunit interact, resulting in Hif-1 heterodimer, which is an active transcription factor. ${ }^{[69]}$ One of the target genes of Hif-1 is vascular endothelial growth factor (VEGF) (see Figure 2). VEGF is increased in bone marrow of MPN patients, particularly in PV and PMF patients. ${ }^{[63,64,70,71]}$ STAT3 activation induces up-regulation of VEGF (see Figure 2), moreover when STAT3 is blocked, VEGF expression is inhibited. ${ }^{\left[{ }^{[2]}\right.}$ An increased phosphorylation of STAT3 might therefore be one of the mechanisms of the increased angiogenesis seen in the bone marrow of MPN patients. However, megakaryocytes and thrombocytes are also important sources of VEGF, since megakaryocytes and thrombocytes are increased in ET, PV and PMF patients, the precise mechanism of increased angiogenesis in MPN patients is not fully understood. ${ }^{[71]} \mathrm{Bcl}$-2/adenovirus E1B $19 \mathrm{kDa}$-interacting protein 3 (Bnip3), another target protein of Hif-1 (see Figure 2), is a pro-apoptotic protein belonging to the BH3-only subfamily activated under hypoxic conditions in normal and cancer tissue by Hif-1 activation. ${ }^{[77-79]}$ In an immunohistochemistry study a trend of lower Bnip3 expression in PMF patients is shown, with a further decrease of the expression in PMF follow-up biopsies. ${ }^{[80]}$ Also, down-regulation of Bnip3 due to DNA methylation and histone deacetylation is shown 
in acute lymphatic leukaemia (ALL), acute myeloid leukaemia (AML) and multiple myeloma (MM). ${ }^{[81]}$ These studies also indicate disturbed apoptosis mechanisms and not only an increased proliferative activity in MPN patients. Further, these studies might also indicate an angiogenesis pathway independent of hypoxia and thus independent of Hif-1 activation, while Bnip3 is expressed under hypoxic conditions.

Table 1: Somatic mutations in ET, PV, PMF and blast phase MPN patients.

\begin{tabular}{|c|c|c|c|}
\hline Mutations & \multicolumn{2}{|c|}{$\begin{array}{l}\text { Mutational } \\
\text { frequency }\end{array}$} & $\begin{array}{l}\text { Pathogenetic } \\
\text { relevance }\end{array}$ \\
\hline $\begin{array}{l}\text { JAK2 }{ }^{\text {V617F }} \text { exon } 14 \\
\text { (janus kinase 2) }\end{array}$ & $\begin{array}{r}\text { PV } \\
\text { ET and PMF } \\
\text { BP-MPN }\end{array}$ & $\begin{array}{r}>95 \% \\
50-60 \% \\
\pm 50 \%\end{array}$ & $\begin{array}{l}\text { abnormal myeloproliferation and progenitor cell } \\
\text { growth factor } \\
\text { hypersensitivity }\end{array}$ \\
\hline $\begin{array}{l}\text { JAK2 exon } 12 \\
\text { MPL (myeloproliferative } \\
\text { leukemia virus } \\
\text { oncogene) }\end{array}$ & $\begin{array}{r}\text { PV } \\
\text { ET } \\
\text { PMF } \\
\text { BP-MPN }\end{array}$ & $\begin{array}{r} \pm 3 \% \\
\pm 3-5 \% \\
\pm 10 \% \\
\pm 5 \%\end{array}$ & $\begin{array}{l}\text { mainly erythroid myeloproliferation } \\
\text { cytokine-independent growth and thrombopoietin } \\
\text { hypersensitivity }\end{array}$ \\
\hline LNK & $\begin{array}{r}E T, P V \text { and PMF } \\
\text { BP-MPN }\end{array}$ & $\begin{array}{r}\text { rare } \\
\pm 10 \%\end{array}$ & wild-type LNK inhibits activity of JAK2 ${ }^{\mathrm{V} 617 \mathrm{~F}}$ and MPL \\
\hline $\begin{array}{l}\text { TET2 (TET oncogene } \\
\text { family member 2) }\end{array}$ & $\begin{array}{r}E T \\
P V \\
P M F \\
\text { BP-MPN }\end{array}$ & $\begin{array}{r}4-5 \% \\
10-16 \% \\
8-14 \% \\
\pm 17 \%\end{array}$ & $\begin{array}{l}\text { catalyzes conversion of } 5 \text {-methylated cytosine } \\
\text { into hydroxy-methylated cytosine and } \\
\text { is therefore a key player in active } \\
\text { DNA demethylation }\end{array}$ \\
\hline $\begin{array}{l}\text { ASXL1 ladditionale sex } \\
\text { combs-like 1) }\end{array}$ & $\begin{array}{r}\text { ET and PV } \\
\text { PMF } \\
\text { BP-MPN }\end{array}$ & $\begin{aligned} & \text { rare } \\
\pm & 13 \% \\
\pm & 18 \%\end{aligned}$ & $\begin{array}{l}\text { wild-type ASXL1 is involved in normal } \\
\text { haematopoiesis and can } \\
\text { act as activator and repressor of transcription }\end{array}$ \\
\hline $\begin{array}{l}\text { IDH1/IDH2 (isocitrate } \\
\text { dehydrogenase) }\end{array}$ & $\begin{array}{r}E T, P V \text { and } P M F \\
\text { BP-MPN }\end{array}$ & $\begin{array}{r}\text { rare } \\
\pm 20 \%\end{array}$ & $\begin{array}{l}\text { converts } \alpha \text {-ketoglutarate into } \\
\text { 2-hydroxyglutarate }(2 \mathrm{HG}) \text { and inhibits } \\
\text { TET2 activity leading to decrease in demethylation }\end{array}$ \\
\hline $\begin{array}{l}\text { EZH2 (enhancer of zeste } \\
\text { homolog 2) }\end{array}$ & $\begin{array}{l}\text { PV } \\
\text { MF }\end{array}$ & $\begin{array}{r} \pm 3 \% \\
\pm 6-13 \%\end{array}$ & $\begin{array}{l}\text { wild-type EZH2 is part of polycomb } \\
\text { repressive complex } 2 \text { (PRC2) }\end{array}$ \\
\hline $\begin{array}{l}\text { DNMT3A (DNA cytosine } \\
\text { methyltransferase } 3 a \text { ) }\end{array}$ & $\begin{array}{r}\mathrm{ET} \\
\mathrm{PV} \\
\mathrm{PMF} \\
\mathrm{BP}-\mathrm{MPN}\end{array}$ & $\begin{array}{r} \pm 3 \% \\
\pm 7 \% \\
\pm 10 \% \\
\pm 15 \%\end{array}$ & $\begin{array}{l}\text { DNA methyltransferase responsibe } \\
\text { for the maintainance of } \\
\text { DNA in the methylated state }\end{array}$ \\
\hline
\end{tabular}

ET: essential thrombocythemia, PV: polycythemia vera, PMF: primary myelofibrosis, BP-MPN: blast phase myeloproliferative, MF: myelofibrosis and includes PMF, post-ET/PV myelofibrosis 
Figure 3: Overview of bone marrow with myelofibrosis.
A. Reticuline fibrosis in bone marrow $(1000 x, \mathrm{HE})$
B. Primary myelofibrosis (1000x, HE).
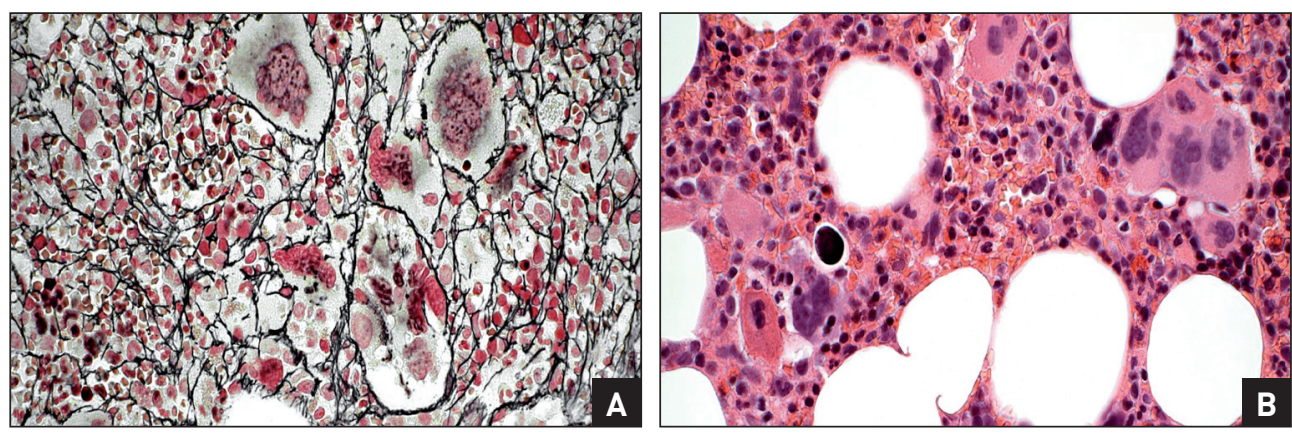

\section{THE PATHOGENESIS OF FIBROSIS}

The amount of vessels is correlated with the grading of fibrosis; the higher the MVD, the more severe the grading of fibrosis, indicating a correlation between angiogenesis and fibrosis. ${ }^{[63,64,66]}$ Myelofibrosis is the abnormal increase in bone marrow fibre content. There is a difference between reticulin fibrosis and collagen fibrosis with the latter showing a stronger correlation with abnormal blood counts and no response to treatment, ${ }^{[82,83]}$ therefore fibrosis is classified in:

- MF-0; single scattered reticulin fibers with no intersections (cross-overs) with the appearance of normal bone marrow.

- MF-1; a loose network of thin reticulin fibers with many intersections, especially in perivascular areas.

- MF-2; a diffuse and dense increase in reticulin forming with extensive intersections, occasionally with only focal bundles of thick collagen fibers and/or focal osteosclerosis.

- MF-3, a diffuse and dense increase in reticulin with extensive intersections with coarse bundles of collagen and often associated with significant osteosclerosis. ${ }^{[84]}$

This classification is a subjective scoring system with limitations, including interobserver variability and inconsistency of use. Further, in daily practice it remains difficult whether a case is MF-0 or MF-1 and MF-2 or MF-3, how much collagen is tolerable to diagnose a case as MF-2. The same is true for ET and the prefibrotic form of PMF which shows phenotypic similarities. ${ }^{[85-87]}$ 
However, the presence and grading of fibrosis is important to diagnose MPN, but it is also a guide for treatment decisions.

Bone marrow fibrosis is a major feature of PMF patients, but can also develop in ET and PV patients, known as post-essential thrombocythemia myelofibrosis (post-ET MF) and post-polycythemia vera myelofibrosis (post-PV MF), respectively. ${ }^{[12,88]}$ Myelofibrosis is characterized in the peripheral blood by tear-drop erythrocytes and the presence of myeloid precursor cells. The symptoms these patients display consist of splenomegaly, progressive anaemia and constitutional symptoms like fatigue, weight loss, night sweats, bone pain and shortness of breath. Survival in myelofibrosis varies from 2 to 11 years due to an increased risk of leukemic transformation. Treatment of myelofbrosis is mainly palliative; blood transfusion, radiotherapy, chemotherapy, splenectomy or stem cell transplantation. The latter is the only curative option. ${ }^{[89]}$

The development of fibrosis has been studied widely, although a clear underlying mechanism has not been identified yet. Platelet-derived growth factor (PDGF) is a potent stimulator of fibroblast growth present in megakaryocytes and platelets, ${ }^{[90]}$ resulting in abnormal numbers of megakaryocytes and platelets in disorders with increased bone marrow fibrosis. Although PDGF stimulates fibroblast growth, studies from Kimura et al. and Terui et al., showed little effect of PDGF on reticulin or collagen production. ${ }^{\text {[91, 92] }}$

Transforming growth factor $\beta$ (TGF- $\beta$ ) is another potent stimulator of fibroblast collagen synthesis found in megakaryocytes and platelets. There are 3 isoforms of TGF- $\beta$; TGF- $\beta 1$, $-\beta 2$ and $-\beta 3$. Megakaryocytes in humans produce and are negatively regulated by, TGF- $\beta 1$. It is shown that megakaryocyte colony-forming units (CFU-MK) in ET patients are less sensitive for the negative regulation of TGF- $\beta 1$ due to a reduced expression of Smad4 lan intracellular effector of TGF- $\beta$ ). ${ }^{[93,94]}$ In PV and PMF patients it was shown that one of the TGF- $\beta$ high-affinity cell surface receptors, T $\beta R I I$, has a reduced expression, resulting in loss of sensitivity to TGF- $\beta 1$ with resistance to its growth-inhibitor and apoptotic effect, allowing clonal expansion of megakaryocytes and fibroblasts. ${ }^{[95-98]}$

In mice models it was shown that megakaryocytes and leucocytes are both responsible in the development of fibrosis. A pathological emperipolesis of polymorphonuclear (PMN) leucocytes within the megakaryocytes results in the destruction of megakaryocyte organelles and leakage of PDGF and TGF- $\beta$ into the bone marrow microenvironment. This indicates that there is an inappropriate release of stored substances in megakaryocytes. The net result is activation and proliferation of fibroblasts leading to fibrosis. This phenomenon is supported by the correlation between the amount of reticulin deposition and the rate of emperipolesis. ${ }^{[99,100]}$ However, it remains unknown what drives the emperipolesis. Other cytokines and haematopoietic growth factors and the interplay between them might be involved in the development of fibrosis in the bone marrow of MPN patients. 


\section{CONCLUDING REMARKS}

Although important progress has been made in the understanding of the MPN pathogenesis due to the discovery of the JAK2 ${ }^{\text {V617F }}$ mutation and the discovery of other mutations, the underlying mechanism of the MPN is still not elucidated. The development of JAK inhibitors has improved the quality of life of MPN patients significantly, although a survival advantage has not been proven yet due to too short follow up time and the data are based on small series.

Although progress in the understanding of the pathophysiological mechanism of fibrosis has been made the last couple of years, discussion remains whether bone marrow fibrosis in MPN patients is of clonal MPN origin, or reactive and polyclonal. However, the most important question remains: what is the main underlying mechanism driving the development of fibrosis? Only when we have the question to this answer can the prognosis and survival of MPN patients can be prolonged. Therefore, more research needs to be done to elucidate the difficult underlying mechanism of myelofibrosis in order to generate better therapeutic options. 


\section{REFERENCES}

1. Vaquez, H., Sur une forme spéciale de cyanose s'accompagnant d'hyperglobulie excessive et persistant. CR Soc Biol, 1892. 44: p. 384-388.

2. Dameshek, W., Some speculations on the myeloproliferative syndromes. Blood, 1951. 6(4): p. 372-5.

3. Baxter, E.J., et al., Acquired mutation of the tyrosine kinase JAK2 in human myeloproliferative disorders. Lancet, 2005. 365(9464): p. 1054-61.

4. James, C., et al., A unique clonal JAK2 mutation leading to constitutive signalling causes polycythaemia vera. Nature, 2005. 434(7037): p. 1144-8.

5. Kralovics, R., et al., A gain-of-function mutation of JAK2 in myeloproliferative disorders. N Engl J Med, 2005. 352(17): p. 1779-90.

6. Levine, R.L., et al., Activating mutation in the tyrosine kinase JAK2 in polycythemia vera, essential thrombocythemia, and myeloid metaplasia with myelofibrosis. Cancer Cell, 2005. 7(4): p. 387-97.

7. Nowell, P.C. and D.A. Hungerford, Chromosome studies on normal and leukemic human leukocytes. J Natl Cancer Inst, 1960. 25: p. 85-109.

8. Fialkow, P.J., S.M. Gartler, and A. Yoshida, Clonal origin of chronic myelocytic leukemia in man. Proc Natl Acad Sci U S A, 1967. 58(4): p. 1468-71.

9. Adamson, J.W., et al., Polycythemia vera: stem-cell and probable clonal origin of the disease. N Engl J Med, 1976. 295(17): p. 913-6.

10. Jacobson, R.J., A. Salo, and P.J. Fialkow, Agnogenic myeloid metaplasia: a clonal proliferation of hematopoietic stem cells with secondary myelofibrosis. Blood, 1978. 51(2): p. 189-94.

11. Fialkow, P.J., et al., Evidence that essential thrombocythemia is a clonal disorder with origin in a multipotent stem cell. Blood, 1981. 58(5): p. 916-9.

12. Vardiman, J.W., et al., The 2008 revision of the World Health Organization (WHO) classification of myeloid neoplasms and acute leukemia: rationale and important changes. Blood, 2009. 114(5): p. 937-51.

13. Campbell, P.J. and A.R. Green, The myeloproliferative disorders. N Engl J Med, 2006. 355(23): p. 2452-66.

14. Murray, J., Myeloproliferative disorders. Clin Med, 2005. 5(4): p. 328-32.

15. Michiels, J.J., et al., Current diagnostic criteria for the chronic myeloproliferative disorders (MPD) essential thrombocythemia (ET), polycythemia vera (PV) and chronic idiopathic myelofibrosis (CIMF). Pathol Biol (Paris), 2007. 55(2): p. 92-104.

16. Rawlings, J.S., K.M. Rosler, and D.A. Harrison, The JAK/STAT signaling pathway. J Cell Sci, 2004. 117(Pt 8): p. 1281-3.

17. Yu, H. and R. Jove, The STATs of cancer--new molecular targets come of age. Nat Rev Cancer, 2004. 4(2): p. 97-105.

18. Darnell, J.E., Jr., STATs and gene regulation. Science, 1997. 277(5332): p. 1630-5.

19. Larsen, L. and C. Ropke, Suppressors of cytokine signalling: SOCS. APMIS, 2002. 110(12): p. 833-44.

20. Valentino, L. and J. Pierre, JAK/STAT signal transduction: regulators and implication in hematological malignancies. Biochem Pharmacol, 2006. 71(6): p. 713-21.

21. Espert, L., I. Dusanter-Fourt, and M.K. Chelbi-Alix, [Negative regulation of the JAK/STAT: pathway implication in tumorigenesis]. Bull Cancer, 2005. 92(10): p. 845-57.

22. Toyama, K., et al., JAK2-V617F mutation analysis of granulocytes and platelets from patients with chronic myeloproliferative disorders: advantage of studying platelets. Br J Haematol, 2007. 139(1): p. 64-9. 
23. Michiels, J.J. and Thiele, J., Artsenbrochure Myeloproliferative Disorders. 2005.

24. Tiedt, R., et al., Ratio of mutant JAK2 ${ }^{\mathrm{V} 617 \mathrm{~F}}$ to wild-type JAK2 determines the MPD phenotypes in transgenic mice. Blood, 2008. 111(8): p. 3931-40.

25. Vannucchi, A.M., et al., Clinical correlates of JAK2 $2^{\mathrm{V} 617 \mathrm{~F}}$ presence or allele burden in myeloproliferative neoplasms: a critical reappraisal. Leukemia, 2008. 22(7): p. 1299-307.

26. Vannucchi, A.M., et al., Clinical profile of homozygous JAK2 $617 \mathrm{~V}>\mathrm{F}$ mutation in patients with polycythemia vera or essential thrombocythemia. Blood, 2007. 110(3): p. 840-6.

27. Passamonti, F. and Rumi, E., Clinical relevance of JAK2 (V617F) mutant allele burden. Haematologica, 2009. 94(1): p. 7-10.

28. Barosi, G., et al., JAK2 ${ }^{\mathrm{V} 617 F}$ mutational status predicts progression to large splenomegaly and leukemic transformation in primary myelofibrosis. Blood, 2007. 110(12): p. 4030-6.

29. Tefferi, A., et al., Low JAK2 ${ }^{\mathrm{V} 617 \mathrm{~F}}$ allele burden in primary myelofibrosis, compared to either a higher allele burden or unmutated status, is associated with inferior overall and leukemia-free survival. Leukemia, 2008. 22(4): p. 756-61.

30. Delhommeau, F., et al., Mutation in TET2 in myeloid cancers. N Engl J Med, 2009. 360(22): p. 2289-301.

31. Pardanani, A., et al., Host genetic variation contributes to phenotypic diversity in myeloproliferative disorders. Blood, 2008. 111(5): p. 2785-9.

32. Stein, B.L., et al., Sex differences in the JAK2 V617F allele burden in chronic myeloproliferative disorders. Haematologica, 2010. 95(7): p. 1090-7.

33. Beer,P.A., etal., Two routes to leukemic transformation after a JAK2 mutation-positive myeloproliferative neoplasm. Blood, 2010. 115(14): p.2891-900.

34. Verstovsek, S., Mesa, R.A., Gotlib, J., A double-blind, placebo-controlled trial of ruxolitinib for myelofibrosis. N Engl J Med, 2012. 366(9): p. 799-807.

35. Harrison, C.N., Kiladjian, J., Al-Ali, H.K., et al., JAK inhibition with ruxolitinib versus best available therapy for myelofibrosis. N Engl J Med, 2012. 366(9): p. 787-98.

36. Ostojic, A., R. Vrhovac, and Verstovsek, S., Ruxolitinib: a new JAK1/2 inhibitor that offers promising options for treatment of myelofibrosis. Future Oncol, 2011. 7(9): p. 1035-43.

37. Pardanani, A., et al., Safety and efficacy of TG101348, a selective JAK2 inhibitor, in myelofibrosis. J Clin Oncol, 2011. 29(7): p. 789-96.

38. Pardanani, A. and Tefferi, A., Targeting myeloproliferative neoplasms with JAK inhibitors. Curr Opin Hematol, 2011. 18(2): p. 105-10.

39. Tefferi, A., and Pardanani A., Serious adverse events during Ruxolitinib treatment disontinuation in patients with myelofibrosis. Mayo Clin Pro, 2011. 86(12): p. 1188-91.

40. Bromberg, J.F., et al., Stat3 as an oncogene. Cell, 1999. 98(3): p. 295-303.

41. Fukada, T., et al., Two signals are necessary for cell proliferation induced by a cytokine receptor gp130: involvement of STAT3 in anti-apoptosis. Immunity, 1996. 5(5): p. 449-60.

42. Kirito, K., et al., A functional role of Stat3 in in vivo megakaryopoiesis. Blood, 2002. 99(9): p. 3220-7.

43. Socolovsky, M., et al., Fetal anemia and apoptosis of red cell progenitors in Stat5a-/-5b-/- mice: a direct role for Stat5 in Bcl-X(L) induction. Cell, 1999. 98(2): p. 181-91.

44. Matsumura, I., et al., Transcriptional regulation of the cyclin D1 promoter by STAT5: its involvement in cytokine-dependent growth of hematopoietic cells. EMBO J, 1999. 18(5): p. 1367-77.

45. Grimwade, L.F., et al., Phospho-STAT5 and phospho-Akt expression in chronic myeloproliferative neoplasms. Br J Haematol, 2009. 147(4): p. 495-506. 
46. Bjornsti, M.A. and Houghton, P.J., The TOR pathway: a target for cancer therapy. Nat Rev Cancer, 2004. 4(5): p. 335-48.

47. Huang, J. and Manning, B.D., A complex interplay between Akt, TSC2 and the two mTOR complexes. Biochem Soc Trans, 2009. 37(Pt 1): p. 217-22.

48. SSkorski, T., et al., Transformation of hematopoietic cells by BCR/ABL requires activation of a PI-3k/ Akt-dependent pathway. EMBO J, 1997. 16(20): p. 6151-61.

49. Kashii, Y., et al., A member of Forkhead family transcription factor, FKHRL1, is one of the downstream molecules of phosphatidylinositol 3-kinase-Akt activation pathway in erythropoietin signal transduction. Blood, 2000. 96(3): p. 941-9.

50. Bakin, A.V., et al., Phosphatidylinositol 3-kinase function is required for transforming growth factor beta-mediated epithelial to mesenchymal transition and cell migration. J Biol Chem, 2000. 275(47): p. 36803-10.

51. Nyga, R., et al., Activated STAT5 proteins induce activation of the PI 3-kinase/Akt and Ras/MAPK pathways via the Gab2 scaffolding adapter. Biochem J, 2005. 390(Pt 1): p. 359-66.

52. Schwaller, J., et al., Stat5 is essential for the myelo- and lymphoproliferative disease induced by TEL/ JAK2. Mol Cell, 2000. 6(3): p. 693-704.

53. Datta, S.R., Brunet, A. and Greenberg, M.E., Cellular survival: a play in three Akts. Genes Dev, 1999. 13(22): p. 2905-27.

54. Vivanco, I. and Sawyers, C.L., The phosphatidylinositol 3-Kinase AKT pathway in human cancer. Nat Rev Cancer, 2002. 2(7): p. 489-501.

55. Datta, S.R., et al., Akt phosphorylation of BAD couples survival signals to the cell-intrinsic death machinery. Cell, 1997. 91(2): p. 231-41.

56. del Peso, L., et al., Interleukin-3-induced phosphorylation of BAD through the protein kinase Akt. Science, 1997. 278(5338): p. 687-9.

57. Cardone, M.H., et al., Regulation of cell death protease caspase-9 by phosphorylation. Science, 1998. 282(5392): p. 1318-21.

58. Laubach, J.P., et al., Polycythemia vera erythroid precursors exhibit increased proliferation and apoptosis resistance associated with abnormal RAS and PI3K pathway activation. Exp Hematol, 2009. 37(12): p. 1411-22.

59. Dai, C., I.J. Chung, and Krantz, S.B., Increased erythropoiesis in polycythemia vera is associated with increased erythroid progenitor proliferation and increased phosphorylation of Akt/PKB. Exp Hematol, 2005. 33(2): p. 152-8.

60. Boulton, T.G., et al., An insulin-stimulated protein kinase similar to yeast kinases involved in cell cycle control. Science, 1990. 249(4964): p. 64-7.

61. Matsuda, S., Gotoh, Y. and Nishida, E., Signaling pathways mediated by the mitogen-activated protein (MAP) kinase kinase/MAP kinase cascade. J Leukoc Biol, 1994. 56(5): p. 548-53.

62. Zha, J., et al., Serine phosphorylation of death agonist BAD in response to survival factor results in binding to 14-3-3 not BCL-X(L). Cell, 1996. 87(4): p. 619-28.

63. Lundberg, L.G., et al., Bone marrow in polycythemia vera, chronic myelocytic leukemia, and myelofibrosis has an increased vascularity. Am J Pathol, 2000. 157(1): p. 15-9.

64. Medinger, M., et al., Angiogenesis and vascular endothelial growth factor-/receptor expression in myeloproliferative neoplasms: correlation with clinical parameters and JAK2-V617F mutational status. Br J Haematol, 2009. 146(2): p. 150-7. 
65. Boiocchi, L., et al., Increased expression of vascular endothelial growth factor receptor 1 correlates with VEGF and microvessel density in Philadelphia chromosome-negative myeloproliferative neoplasms. J Clin Pathol, 2011. 64(3): p.226-31.

66. Boveri, E., et al., Bone marrow microvessel density in chronic myeloproliferative disorders: a study of 115 patients with clinicopathological and molecular correlations. Br J Haematol, 2008. 140(2): p. 162-8.

67. Medinger, M., N. Fischer, and A. Tzankov, Vascular endothelial growth factor-related pathways in hemato-lymphoid malignancies. J Oncol. 2010: p. 729725.

68. Folkman, J., Angiogenesis in cancer, vascular, rheumatoid and other disease. Nat Med, 1995. 1(1): p. 27-31.

69. Nakayama, K., Cellular signal transduction of the hypoxia response. J Biochem, 2009. 146(6): p. 757-65.

70. Gianelli, U., et al., VEGF expression correlates with microvessel density in Philadelphia chromosomenegative chronic myeloproliferative disorders. Am J Clin Pathol, 2007. 128(6): p. 966-73.

71. Wrobel, T., et al., Increased expression of vascular endothelial growth factor (VEGF) in bone marrow of patients with myeloproliferative disorders (MPD). Pathol Oncol Res, 2003. 9(3): p. 170-3.

72. Niu, G., et al., Constitutive Stat3 activity up-regulates VEGF expression and tumor angiogenesis. Oncogene, 2002. 21(13): p. 2000-8.

73. Milosevic, J.D., Kralovics, R., Genetic and epigenetic alterations of myeloproliferative disorders. Int J. Hematol, 2013. 97(2): p. 183-97.

74. Brecqueville, M., et al., Mutation analysis of ASXL1, CBL, DNMT3A, IDH1, IDH2, JAK2, MPL, NF1, SF3B1, SUZ12, and TET2 in Myeloproliferative Neoplasms. Genes, chromosomes and cancer, 2012. 51(8): p. 743-55.

75. Porcu, M., et al., Mutation of the receptor tyrosine phosphatase PTPRC (CD45) in T-cell acute lymphoblastic leukemia. Blood, 2012. 119(19): p. 4476-9.

76. Trelinski, J., Robak, T., JAK inhibitors: pharmacology and clinical activity in chronic myeloprolipherative neoplasms. Curr Med Chem, 2013. 20(9): p. 1147-61.

77. Boyd, J.M., et al., Adenovirus E1B $19 \mathrm{kDa}$ and Bcl-2 proteins interact with a common set of cellular proteins. Cell, 1994. 79(2): p. 341-51.

78. Chen, G., et al., The E1B 19K/Bcl-2-binding protein Nip3 is a dimeric mitochondrial protein that activates apoptosis. J Exp Med, 1997. 186(12): p. 1975-83.

79. Giatromanolaki, A., et al., BNIP3 expression is linked with hypoxia-regulated protein expression and with poor prognosis in non-small cell lung cancer. Clin Cancer Res, 2004. 10(16): p. 5566-71.

80. Theophile, K., et al., Expression profiling of apoptosis-related genes in megakaryocytes: BNIP3 is downregulated in primary myelofibrosis. Exp Hematol, 2008. 36(12): p. 1728-38.

81. Murai, M., et al., Aberrant DNA methylation associated with silencing BNIP3 gene expression in haematopoietic tumours. Br J Cancer, 2005. 92(6): p. 1165-72.

82. Kuter, D.J., et al., Bone marrow fibrosis: pathophysiology and clinical significance of increased bone marrow stromal fibres. Br J Haematol, 2007. 139(3): p. 351-62.

83. Thiele, J. and Kvasnicka, H.M., Grade of bone marrow fibrosis is associated with relevant hematological findings-a clinicopathological study on 865 patients with chronic idiopathic myelofibrosis. Ann Hematol, 2006. 85(4): p. 226-32.

84. Thiele, J., et al., European consensus on grading bone marrow fibrosis and assessment of cellularity. Haematologica, 2005. 90(8): p. 1128-32. 
85. Thiele, J., et al., Idiopathic primary osteo-myelofibrosis: a clinico-pathological study on 208 patients with special emphasis on evolution of disease features, differentiation from essential thrombocythemia and variables of prognostic impact. Leuk Lymphoma, 1996. 22(3-4): p. 303-317.

86. Thiele, J., et al., Clinicopathological diagnosis and differential criteria of thrombocythemias in various myeloproliferative disorders by histopathology, histochemistry and immunostaining from bone marrow biopsies. Leuk Lymphoma, 1999. 33(3-4): p.207-218.

87. Thiele, J, . et al., Initial (prefibrotic) stages of idiopathic (primary) myelofibrosis (IMF) - a clinicopathological study. Leukemia, 1999. 13(11): p.1741-1748.

88. Mesa, R.A., et al., Primary myelofibrosis (PMF), post polycythemia vera myelofibrosis (post-PV MF), post essential thrombocythemia myelofibrosis (post-ET MF), blast phase PMF (PMF-BP): Consensus on terminology by the international working group for myelofibrosis research and treatment (IWGMRT). Leuk Res, 2007. 31(6): p. 737-40.

89. Tefferi, A., Myelofibrosis with myeloid metaplasia. N Engl J Med, 2000. 342(17): p. 1255-65.

90. Groopman, J.E., The pathogenesis of myelofibrosis in myeloproliferative disorders. Ann Intern Med, 1980. 92(6): p. 857-8.

91. Kimura, A., et al., Transforming growth factor-beta regulates growth as well as collagen and fibronectin synthesis of human marrow fibroblasts. Br J Haematol, 1989. 72(4): p. 486-91.

92. Terui, T., et al., The production of transforming growth factor-beta in acute megakaryoblastic leukemia and its possible implications in myelofibrosis. Blood, 1990. 75(7): p. 1540-8.

93. Dong, M. and G.C. Blobe, Role of transforming growth factor-beta in hematologic malignancies. Blood, 2006. 107(12): p. 4589-96.

94. Kuroda, H., et al., Decrease of Smad4 gene expression in patients with essential thrombocythaemia may cause an escape from suppression of megakaryopoiesis by transforming growth factor-beta1. Br J Haematol, 2004. 124(2): p. 211-20.

95. Le Bousse-Kerdiles, M.C., et al., Differential expression of transforming growth factor-beta, basic fibroblast growth factor, and their receptors in CD34+ hematopoietic progenitor cells from patients with myelofibrosis and myeloid metaplasia. Blood, 1996. 88(12): p. 4534-46.

96. Rooke, H.M., et al., The TGF-beta type II receptor in chronic myeloid leukemia: analysis of microsatellite regions and gene expression. Leukemia, 1999. 13(4): p. 535-41.

97. Wang, J.C., et al., Quantitative analysis of growth factor production in the mechanism of fibrosis in agnogenic myeloid metaplasia. Exp Hematol, 2006. 34(12): p. 1617-23.

98. Chou, J.M., C.Y. Li, and A. Tefferi, Bone marrow immunohistochemical studies of angiogenic cytokines and their receptors in myelofibrosis with myeloid metaplasia. Leuk Res, 2003. 27(6): p. 499-504.

99. Schmitt, A., et al., Pathologic interaction between megakaryocytes and polymorphonuclear leukocytes in myelofibrosis. Blood, 2000. 96(4): p. 1342-46.

100. Schmitt, A., et al., Polymorphonuclear neutrophil and megakaryocyte mutual involvement in myelofibrosis pathogenesis. Leukemia and Lymphoma, 2002. 43(4): p. 719-24. 


\section{REPRODUCIBILITY OF HISTOLOGIC CLASSIFICATION \\ IN NON-FIBROTIC MYELOPROLIFERATIVE NEOPLASIA}

Suzanne M. Koopmans

Freek J. Bot

King H. Lam

Ariënne M.W. van Marion

Hendrik de Raeve

Konnie M. Hebeda

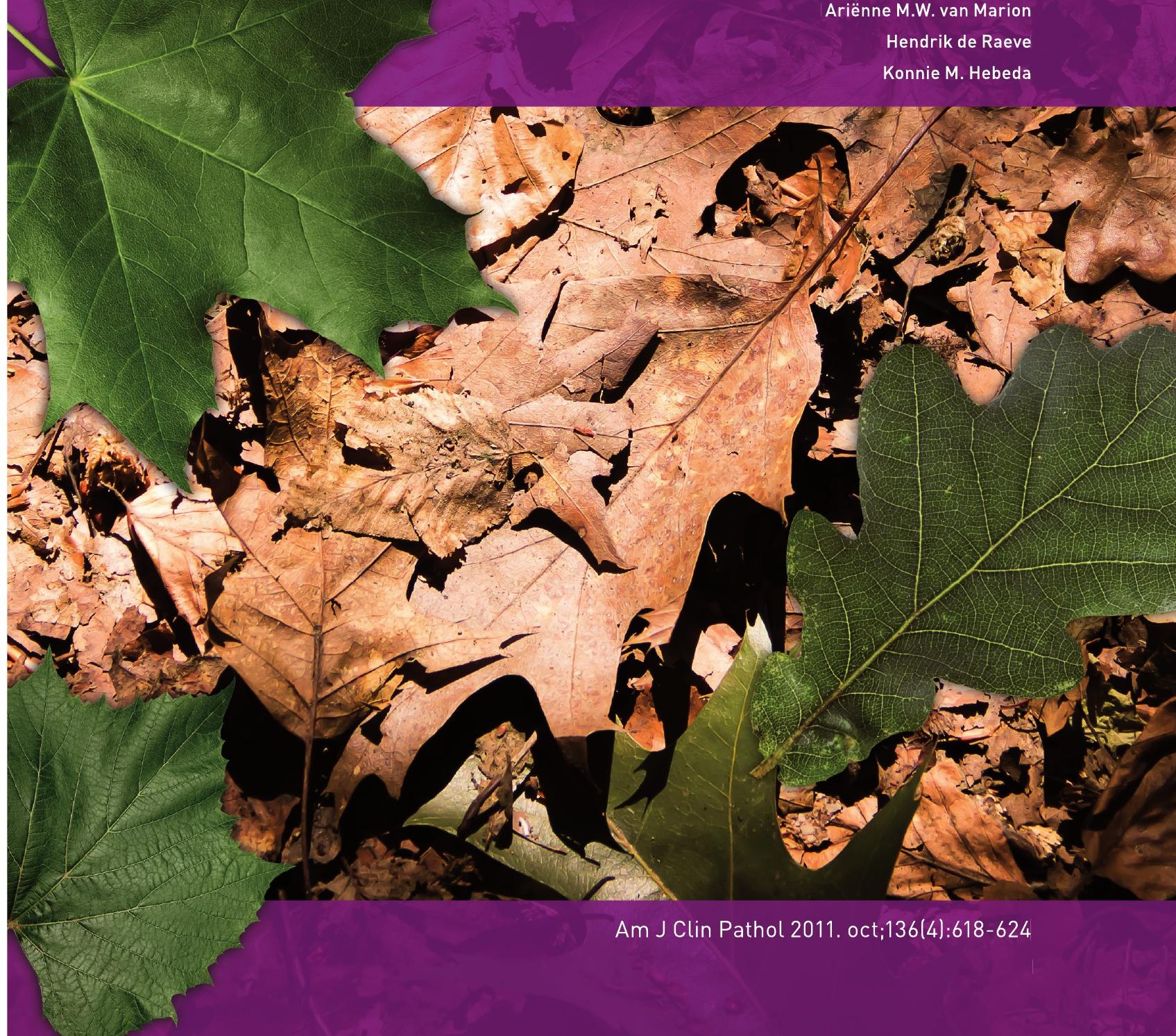




\section{ABSTRACT}

Early phases of polycythemia vera (PV), essential thrombocythemia (ET) and primary myelofibrosis (PMF) can be difficult to distinguish by morphologic studies alone because they share many morphological characteristics. Histological criteria according to the 2008 World Health Organization (WHO) classification are part of Myeloproliferative neoplasia (MPN) diagnosis. Our aim was to assess the reproducibility of morphological characteristics and determine their relative importance for histological diagnosis on selected trephine biopsy sections.

For the study, 56 prefibrotic MPN trephine specimens were blindly reviewed by 4 haematopathologists using a scoring list of 16 histological characteristics mentioned in the WHO classification. Consensus was defined as agreement by 3 out of 4 haematopathologists.

High degrees of consensus were reached for individual major morphological features used in the WHO classification, especially for the nuclear features of megakaryocytes $(83 \%)$. Some of the features correlated positively or negatively with the histological diagnosis of PMF. Consensus for the histological classification of MPN was reached in 39 $(70 \%)$ of 56 cases without knowledge of clinical data. This finding indicates a difference in relative importance assigned to individual histological characteristics by different haematopathologists. 


\section{INTRODUCTION}

Myeloproliferative neoplasms (MPN) are clonal bone marrow stem cell disorders originating from a multipotent haematopoietic stem cell. MPNs are characterized by the proliferation of one or more lineages of myeloid, erythroid and megakaryocytic cell lineages resulting in increased numbers of granulocytes, erythrocytes or platelets in the peripheral blood. According to the 2008 World Health Organization (WHO) criteria, MPNs can be divided into chronic myelogenous leukemia (CML) carrying the Philadelphia $(\mathrm{Ph}+)$ chromosome as a result of $t(9 ; 22)$, resulting in the $B C R-A B L 1$ fusion gene, and diseases that do not carry the Philadelphia chromosome (Ph-). ${ }^{[1]}$ The three most commonly occurring, so called classical $\mathrm{Ph}$ negative MPNs (Ph-MPN) are polycythemia vera (PV), essential thrombocythemia (ET) and primary myelofibrosis (PMF). ${ }^{[2,3]}$

In 1967, the Polycythemia Vera Study Group (PVSG) initiated extensive studies of PV. The diagnostic criteria were updated several times during the following decades and are widely used by haematologists. However, the appropriate use of the histological studies of bone marrow biopsy (BMB) specimens as a diagnostic tool was neglected. To stress the relevance of a BMB, the WHO added a set of histological diagnostic criteria in 2001. ${ }^{[4]}$ The recent discovery of the JAK2 $2^{\mathrm{V} 17 F}$ mutation and the recognition of pre-fibrotic PMF resulted in the 2008 WHO classification of MPNs. ${ }^{[1,5-7]}$

$\mathrm{PV}$ is characterized by a proliferation of the three major haematopoietic cell lineages, usually resulting in increased numbers of circulating erythrocytes and often also leukocytes and blood platelets. The bone marrow features of PV are trilinear hypercellularity, loose clusters of a range of small to giant megakaryocytes, and, sometimes, a slightly increased amount of reticulin fibrosis.

The typical features of ET are thrombotic and haemorrhagic complications due to the proliferation of the megakaryocytic cell line, resulting in trombocythemia. The bone marrow is characterized by increased numbers of loosely clustering, giant, hyperlobated megakaryocytes with staghorn-like features and a lack of reticulin fibrosis. Erythropoiesis and myelopoiesis are typically not involved.

The bone marrow of patients with PMF is characterized by a proliferation of the megakaryocytic and, less conspicuously, granulocytic cell lineage. The megakaryocytes often demonstrate dense clustering and a large range in cell size, including giant megakaryocytes. Their nuclei demonstrate atypical features such as cloud-like aspect, hypolobulation, irregular nuclear outlines, and hyperchromatic chromatin. During the course of the disease, the amount of reticulin fibrosis increases, finally resulting in collagen fibrosis with osteosclerosis. ${ }^{[3,8]}$

Early phases of PV, ET and PMF share many morphological characteristics and consequently can be difficult to distinguish from each other when using only histological evaluation. 
Distinguishing these three MPN subtypes in the early phase reliably is important, because of a different risk of thromboembolic complications of PV and the worse survival rate of PMF patients compared to ET patients, who have a normal life expectancy. ${ }^{[1,9]}$

The first aim of this study was to assess the reproducibility of the major individual morphological characteristics described in the WHO classification for the different prefibrotic MPNs. The other aims were to assess the reproducibility of the histological diagnosis using only morphological characteristics without knowledge of the clinical data and to gain insight into interpathologist differences.

\section{MATERIALS AND METHODS}

\section{Bone marrow trephine specimens}

Diagnostic BMB specimens from 56 consecutive patients diagnosed between 2001 and 2006 as having non-fibrotic ET ( $n=30$ ) or PV ( $n=26)$ according to the PVSG criteria were retrieved from the files of the University Hospital Antwerp, Antwerp, Belgium. Bone marrow trephine biopsy specimens from all patients were routinely embedded in paraffin, and the original diagnostic sections were used for this study. The sections had been stained with haematoxylin and eosin (H\&E), periodic acid-Schiff (PAS) and Gomori silver impregnation to evaluate the morphologic features and reticulin fiber content.

\section{Assessment of bone marrow trephine slides}

The 56 trephine specimens were blindly reviewed by four pathologists (F.J.B., K.H.L., A.M.W.M., and K.M.H.) from different hospitals with a special interest in MPNs. Each pathologist assessed the trephine specimens independently and without knowledge of patients' age, sex or any other clinical data and without knowledge of the original diagnosis. For the study, 16 histological characteristics, mainly related to megakaryocyte morphologic features, were previously agreed on and were scored for each case. An arbitrary threshold of at least $10 \%$ within the cells of a lineage was accepted, although the WHO classification does not give any quantitative criteria. Deliberately, no detailed agreement on the criteria was sought beforehand to establish whether there was consensus in the use of the WHO 2008 histological criteria in daily practice. Megakaryocyte nuclei were scored as staghorn, cloud like, dysmorphic, or bare nuclei. The nuclear lobulation of the megakaryocytes was scored as normal, hyperlobulation, or hypolobulation. The clustering was divided into no clustering, loose clustering, or dense clustering. The cytoplasm of the megakaryocytes was recorded as normal, small, large, or dysmorphic. Additional features were dilated sinusoids and the myeloid/erythroid ratio (M/E ratio) (see Table 1). 
Table 1: Degree of consensus for 16 histological characteristics in 56 cases of myeloproliferative neoplasia*.

\begin{tabular}{|c|c|c|}
\hline & Present & Absent \\
\hline \multicolumn{3}{|l|}{ Megakaryocyte nuclei } \\
\hline $\begin{array}{l}\text { Staghorn } \\
\text { Cloud like } \\
\text { Naked nuclei } \\
\text { Dysmorph }\end{array}$ & $\begin{aligned} 53 & (95[88.6-100.6]) \\
48 & (86[76.0-95.5]) \\
477 & (84[73.8-94.0]) \\
46 & (82[71.4-92.9])\end{aligned}$ & 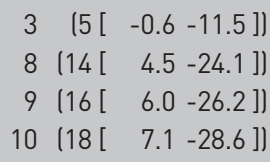 \\
\hline \multicolumn{3}{|l|}{ Lobulation } \\
\hline $\begin{array}{l}\text { Normal } \\
\text { Hyperlobulated } \\
\text { Hypolobulated }\end{array}$ & 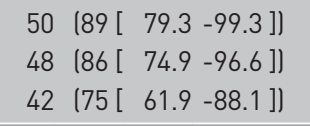 & 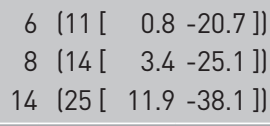 \\
\hline \multicolumn{3}{|l|}{ Clustering } \\
\hline $\begin{array}{l}\text { Normal } \\
\text { Loose } \\
\text { Dense }\end{array}$ & $\begin{array}{ll}41 & (73[60.0-86.5]) \\
40 & (71[57.8-85.1]) \\
49 & (88[78.0-97.0])\end{array}$ & 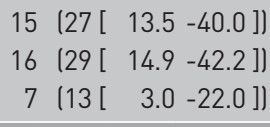 \\
\hline \multicolumn{3}{|c|}{ Megakaryocyte cytoplasm } \\
\hline $\begin{array}{l}\text { Normal } \\
\text { Small } \\
\text { Large } \\
\text { Dysmorph }\end{array}$ & 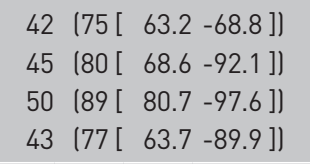 & 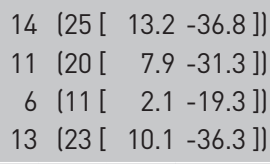 \\
\hline \multicolumn{3}{|l|}{ Other features } \\
\hline $\begin{array}{l}\text { Dilated sinusoids } \\
\text { Myeloid/Erythroid ratio }\end{array}$ & $\left.\left.\begin{array}{llll}48 & (86[ & 75.5 & -95.9\end{array}\right]\right)$ & 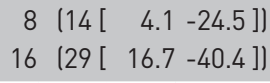 \\
\hline Diagnosis (MPN type) & $39(70[57.6-81.3])$ & $17(30[18.3-42.4])$ \\
\hline
\end{tabular}

* Values are given as number of cases (percentage [95\% confidence interval]).

Definitions of the morphological features are given in Table 2. The histological diagnosis was made according to the WHO 2008 criteria. ${ }^{[1]}$ The diagnosis was no MPN or MPN, and, if possible, was further classified as ET, PV or PMF. Although essential for the final diagnosis of the MPN, we did not record the clinical and laboratory data because this study was about measuring the interobserver variation in evaluating the histological features used in the WHO 2008, irrespective of the clinical and laboratory data. Consensus was defined as agreement by three out of four pathologists. For statistical analysis Excel (Microsoft, Redmond, WA) was used to calculate the percentage of consensus and the confidence intervals.

To study the relative importance of the individual morphological features for the diagnosis, we analyzed their reported frequency in PMF and non-PMF cases for each pathologist. 
Features that were reported as present in at least $75 \%$ of these cases or in fewer than $25 \%$ were considered of diagnostic importance and potentially able to discriminate between the two groups.

Table 2: Definitions of morphological features.

\begin{tabular}{|c|c|}
\hline Morphologic features & Definition \\
\hline Staghorn nuclei & $\begin{array}{l}\text { Large cells with deeply lobulated nuclei } \\
\text { surrounded by mature cytoplasm }\end{array}$ \\
\hline Cloud like nuclei & $\begin{array}{l}\text { Enlarged bulbous, plump nuclei with } \\
\text { decreased amount of cytoplasm }\end{array}$ \\
\hline Naked nuclei & $\begin{array}{l}\text { Compact hyperchromatic megakaryocyte } \\
\text { nuclei without visible cytoplasm }\end{array}$ \\
\hline Dysmorphic nuclei & Hyperchromatic nuclei with bizarre shapes \\
\hline Hyperlobulation & $\begin{array}{l}>6 \text { nuclear lobules; lobules often completely } \\
\text { separated by cytoplasm }\end{array}$ \\
\hline Hypolobulation & $<4$ nuclear lobules surrounded by ample mature cytoplasm \\
\hline Dense clustering & $\begin{array}{l}\text { At least } 4 \text { megakaryocytes lying back-to-back, } \\
\text { without being separated by other cells }\end{array}$ \\
\hline Loose clustering & $\begin{array}{l}\text { Dispersed cluster of at least } 3 \text { megakaryocytes } \\
\text { without close contact }\end{array}$ \\
\hline Small megakaryocyte size & Megakaryocytes $<4$ myeloid cells in largest dimension \\
\hline Large megakaryocyte size & Megakaryocytes $>8$ myeloid cells in largest dimension \\
\hline $\begin{array}{l}\text { Dysmorphic megakaryocyte } \\
\text { cytoplasm }\end{array}$ & $\begin{array}{l}\text { Small to large megakaryocytes with abnormal nuclear/ } \\
\text { cytoplasmic ratio and a shape other than round }\end{array}$ \\
\hline Dilated sinusoids & Visible sinusoids that may or may not be filled with haematopoietic cells \\
\hline Myeloid/Erythroid ratio & Ratio of estimated numbers of myeloid and nucleated erythroid cells \\
\hline
\end{tabular}




\section{RESULTS}

Each pathologist scored the presence of 16 histological characteristics and made a histological diagnosis according to the WHO criteria. Some examples are shown in Figure 1. As the scoring date show in Table 1, variation in the degree of consensus was found in the scoring of the 16 histological characteristics, varying from $95 \%$ for the nuclear aspect staghorn to $71 \%$ for the presence of loose clustering of megakaryocytes. The degree of consensus for the nuclear features of the megakaryocytes was relatively high (at least $75 \%$ for hypolobulation), and the consensus for megakaryocyte cytoplasmic characteristics such as large and small was slightly lower. Also, the consensus for dense clustering (88\%) was comparably high in comparison with loose clustering and no clustering ( $71 \%$ and $73 \%$ respectively). Of the other characteristics, the M/E ratio showed lower consensus (71\%, [40/56]); of these 40 cases, $2(5 \%)$ of 40 were diagnosed as erythroid hyperplasia and $37(93 \%)$ of 40 as having a normal $\mathrm{M} / \mathrm{E}$ ratio and in $1(3 \%)$ of the 40 cases, there was consensus about the presence of myeloid hyperplasia. As expected, the degree of consensus for the histological diagnosis of MPN was $100 \%$ (56/56).

The confidence intervals are given in Table 1 along with the degree of consensus for the 16 histological characteristics. The confidence interval has a comparable range for most morphological features.

The consensus frequency for the histological diagnosis of the various subtypes was $80 \%$ (40/56) for PMF, 20\% (11/56) for PV and 0\% (0/56) for ET. PV was considered by at least one pathologist in $24(43 \%)$ and ET only in $7(13 \%)$ of the 56 trephine specimens.

The features that were present in at least $75 \%$ of PMF cases were large megakaryocytes, small megakaryocytes, hyperlobulation, and a normal M/E ratio. Erythroid hyperplasia was reported in fewer than $25 \%$ of the PMF cases. In the non-PMF cases, no single feature was reported in more than $75 \%$, but dysmorphic nuclei and megakaryocytes, dense clustering, dilated sinusoids and myeloid hyperplasia were generally absent $(<25 \%)$. Because myeloid hyperplasia, staghorn nuclei and normal lobulation were reported in fewer than $25 \%$ of cases in both groups, they were not useful for discrimination (see Table 3). 
Figure 1: Examples of morphological features in megakaryocytes.

A. Dysmorphic nucleus (arrow; H\&E, x200) B. Loose clustering (H\&E, x100) C. Hyperlobulated and enlarged nuclei (arrow; H\&E, x200) D. Dense clustering (periodic acid-Schiff, x100) E. Staghorn nucleus (H\&E; x1000) F. Cloud-like nucleus (H\&E; x1000) G. Small megakaryocyte cytoplasm (arrow; H\&E, x1000) H. Dilated sinusoids $(H \& E, x 100)$ I. Dysmorphic megakaryocyte larrow; H\&E, x1000) J. Hypolobulated nuclei larrow; H\&E, x1000) K. Large megakaryocyte cytoplasm (H\&E, x1000) L. Naked megakaryocyte nuclei (arrow; H\&E, x1000).
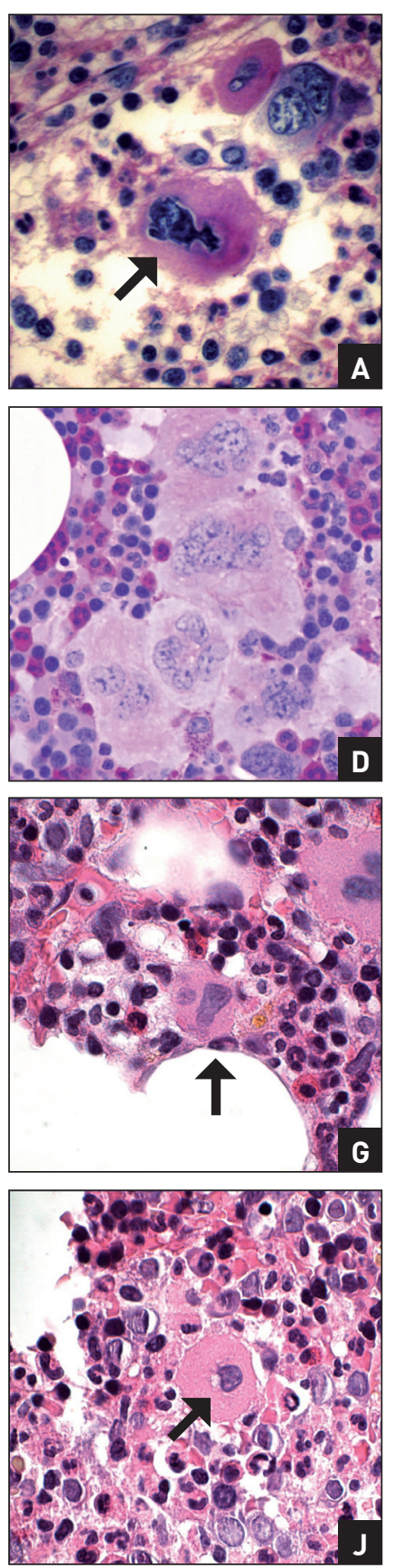
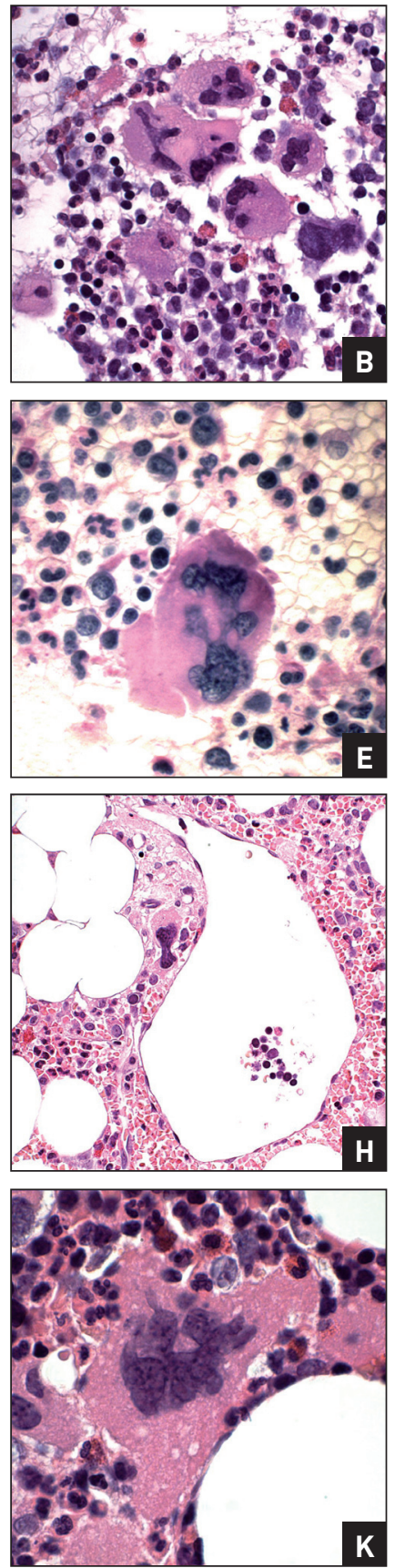
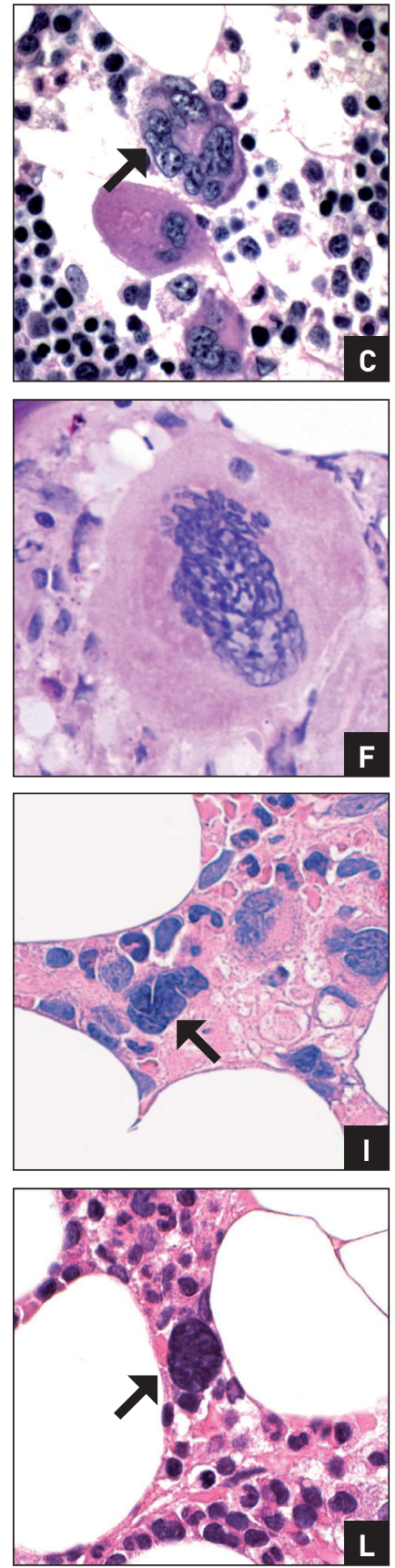
Table 3: Morphologic features recorded by four pathologists in $<25 \%$ or $>75 \%$ of cases considered as PMF compared to non-PMF*.

\begin{tabular}{l|rl|rl}
\hline & \multicolumn{3}{|c|}{ PFM } & Non-PFM \\
\hline Frequent in PMF & & & & \\
\hline Small megakaryocyte cytoplasm & $101 / 134$ & $(75)$ & $46 / 72$ & $(64)$ \\
Large megakaryocyte cytoplasm & $107 / 134$ & $(80)$ & $43 / 69$ & $(62)$ \\
Hyperlobulation & $97 / 129$ & $(75)$ & $48 / 71$ & $(68)$ \\
Normal myeloid/erythroid ratio & $100 / 133$ & $(75)$ & $42 / 78$ & $(55)$ \\
Dysmorphic nuclei & $53 / 130$ & $(41)$ & $12 / 71$ & $(17)$ \\
Dysmorphic megakaryocytes & $61 / 136$ & $(45)$ & $18 / 71$ & $(25)$ \\
Dense clustering & $59 / 134$ & $(44)$ & $9 / 55$ & $(16)$ \\
\hline Rare in non-PMF & & & $13 / 71$ & $(18)$ \\
\hline Frequent in PMF & & & & \\
\hline Rare in PMF & $48 / 137$ & $(35)$ & $28 / 62$ & $(45)$ \\
\hline Erythroid hyperplasia & & & $5 / 61$ & $(8)$ \\
Staghorn & $7 / 140$ & $(5)$ & $18 / 83$ & $(22)$ \\
\hline Normal lobulation & $12 / 122$ & $(10)$ & & \\
\hline Non-discriminatory & $13 / 122$ & $(11)$ & $0 / 0$ & $(0)$ \\
\hline Myeloid hyperplasia & & & $(20)$ &
\end{tabular}

PMF, primary myelofibrosis

* Data are given as number/total (percentage).

\section{DISCUSSION}

In this study, trephine biopsy specimens from 56 patients initially diagnosed as having a nonfibrotic MPN were blindly and independently reviewed by four haematopathologists using a scoring system of 16 histological characteristics. The degree of consensus was relatively high for the overall nuclear features of the megakaryocytes (83\%), calculated as the mean of the ten nuclear features of the megakaryocytes. Especially the degree of consensus for the aspect of the megakaryocyte nuclei was high. These findings indicate that there is rather good agreement among haematopathologists concerning the definition of morphological features. With the clinical PVSG criteria pre-fibrotic PMF was not recognized as a separate entity and was classified as ET or PV. These criteria resulted in a relatively high frequency of ET owing to the presence of trombocythemia that can occur in pre-fibrotic MPN. In our study, the use of the 2008 histological WHO criteria led to a higher frequency of PMF (80\%) and a lower frequency of PV $(20 \%)$ and none of the trephine specimens were diagnosed as ET by consensus, ET was considered in only $13 \%$ of the trephine specimens. In line with our study, similar results have 
been found by the group of Gianelli et al. ${ }^{[10]}$ when they used the WHO criteria to reclassify patients with ET as diagnosed by the PVSG criteria. They found that the diagnosis for only $19 \%$ of the patients remained as ET, whereas the great majority of patients was re-diagnosed as having PMF. Comparable data are shown by Thiele and Kvasnicka ${ }^{[11]}$ and Florena et al..$^{[12]}$ It seems from this and other studies that the clinical manifestations of ET, pre-fibrotic PMF, and early fibrotic PMF are quite similar and that the clinical relevance of the sub classification can not always be demonstrated. ${ }^{[10,11,13,14]}$

Samuelson et al. ${ }^{[14]}$ questioned in a letter to the editor whether there is sufficient confidence that evaluation of megakaryocyte morphologic features and fibrosis is widely reproducible among various observers. The study by Wilkins et al. ${ }^{[9]}$ supports this concern. Although individual morphological features such as megakaryocyte lobulation, size, and clustering, which are important features for differentiating MPNs, show an acceptable degree of consensus for pathologists, this might be insufficient for daily practice in diagnosing MPN subtypes and predicting the differences in clinical outcome and prognosis, especially without further information on the thresholds and weight of these features. As shown in Table 1, consensus was particularly low for the characteristic 'megakaryocyte clustering', except for 'dense clustering'. This finding indicates differences in the perception of 'loose clustering'. Loose clusters of megakaryocytes are considered a feature of ET and PV ${ }^{[1]}$, but apparently it is difficult to distinguish 'loose clusters' from 'no clusters', thus leaving only dense clusters as a discriminatory feature. In our study, dense clustering was only scored in PMF, indicating its weight in diagnosing PMF. Wilkins et al. ${ }^{[9]}$, on the other hand, found it more difficult to distinguish between 'loose clusters' and 'dense clusters', and, in their study, the type of clusters showed a low strength of association. From that finding and our findings it can be concluded that the aspect of clustering of megakaryocytes is difficult to apply reproducibly and that there is a need for providing criteria for determining the type of clustering.

Gianelli et al. ${ }^{[10]}$ showed that the recognition of dysmorphic megakaryocytes is very important, demonstrating that besides dense clustering, dysmorphic features of the megakaryocytes discriminate non-fibrotic PMF from ET. Also, in our data dysmorphic megakaryocytes were scored only in PMF, indicating specific importance in PMF. A low degree of consensus was reached for especially 'normal megakaryocyte size'. The size of the megakaryocytes showed a more acceptable degree of consensus, $80 \%$, but it varied from $75 \%$ for 'normal megakaryocyte size' to $89 \%$ for 'large megakaryocytes'. The low consensus for 'normal' megakaryocytes is partly due to an inconsistency in scoring by some of the pathologists: in case of abundant abnormal megakaryocytes the presence of small numbers of normal megakaryocytes was not always recorded.

Megakaryocytic lobulation showed comparable results, with the degree of consensus of $83 \%$. Hyperlobulation was one of the most common scored characteristic in PMF, as was 
hypolobulation in non-PMF cases, indicating its importance. For the M/E ratio there was a $71 \%$ degree of consensus, and of these cases, $93 \%$ were diagnosed as having a normal M/E ratio. As depicted in the WHO 2008 criteria, the recognition of a significant degree of granulocytic proliferation is important to distinguish PMF from ET. ${ }^{[5]}$ In our study $80 \%$ were re-diagnosed as PMF; however, in only $3 \%$ of these cases was there consensus on myeloid hyperplasia. Also, the normal M/E ratio was one of the four average characteristics scored in PMF. This indicates that granulocytic proliferation is not considered a prerequisite for the diagnosis of PMF; other criteria or combined features were judged to be more important in reaching a diagnosis of PMF.

As one can expect, each morphologic feature makes a different contribution for each diagnosis. The numbers in our study were too small for a confident determination of their relative importance. From the reported frequency in PMF and non-PMF cases, we have at least some information on their assigned importance. Features that are considered as major histological criteria for PMF by the WHO are small to large megakaryocytes with an aberrant nuclear/cytoplasmic ratio and hyperchromatic, bulbous, or irregularly folded nuclei and dense clustering (Table 2.04 from Swerdlow et al. ${ }^{[1]}$. In this study, these features were frequently reported in PMF (large megakaryocytes, small megakaryocytes) or rarely reported in cases diagnosed as non-PMF (dysmorphic nuclei and megakaryocytes, dense clustering). These findings indicate that the latter criteria are specific but apparently not sensitive enough to exclude PMF on their own in individual cases.

The aims of this study were to assess the reproducibility of the morphological characteristics that are used in the WHO 2008 classification and to determine their relative importance for histological diagnosis on selected trephine biopsy sections without knowledge of the clinical data. The independence of the clinical data in this study is important because the histological picture is a major criterion for PMF, a necessary criterion for ET and a minor criterion for PV. Moreover, in daily practice, recognition of a myeloproliferative disorder and histological sub typing have to be performed quite often without all required clinical data to reach a final histological diagnosis. ${ }^{[5,6]}$

Our study showed a high degree of consensus for individual histological features that are described in the WHO classification of MPN BMB specimens, especially concerning the megakaryocytic characteristics. The translation to a final histological diagnosis is more problematic because, besides the recognition of individual histological features, also their frequency, ranking and combination have a role. Future diagnostics for MPN will increasingly integrate clinical and morphological methods with genetic and protein expression data.

A good example is the incorporation of the JAK2 mutation status in MPN diagnostics. However, at least for the time being, histological assessment of a trephine biopsy specimen remains a tool for the sub classification of MPNs in daily clinical practice and in clinical trials. 


\section{REFERENCES}

1. Swerdlow, S.H., et al., WHO Classification of Tumours of Haematopoietic and Lymphoid Tissues, Fourth Edition. WHO Classification of Tumours, Volume 2. IARC WHO Classification of Tumours, No 2. 2008.

2. Campbell, P.J. and A.R. Green, The myeloproliferative disorders. N Engl J Med, 2006. 355(23): p. 2452-66.

3. Murray, J., Myeloproliferative disorders. Clin Med, 2005. 5(4): p. 328-32.

4. Jaffe, E.S., et al., World Health Organization classification of tumours of hematopoietic and lymphoid tissues. Lyon: IARC Press; 2001.

5. Tefferi, A., et al., Proposals and rationale for revision of the World Health Organization diagnostic criteria for polycythemia vera, essential thrombocythemia, and primary myelofibrosis: recommendations from an ad hoc international expert panel. Blood, 2007. 110(4): p. 1092-7.

6. Tefferi, A. and J.W. Vardiman, Classification and diagnosis of myeloproliferative neoplasms: the 2008 World Health Organization criteria and point-of-care diagnostic algorithms. Leukemia, 2008. 22(1): p. 14-22.

7. Turkington, R.C., et al., Comparison of diagnostic criteria for polycythaemia vera. Hematology, 2007. 12(2): p. 123-30.

8. Michiels, J.J., et al., Current diagnostic criteria for the chronic myeloproliferative disorders (MPD) essential thrombocythemia (ET), polycythemia vera (PV) and chronic idiopathic myelofibrosis (CIMF). Pathol Biol (Paris), 2007. 55(2): p. 92-104.

9. Wilkins, B.S., et al., Bone marrow pathology in essential thrombocythemia: interobserver reliability and utility for identifying disease subtypes. Blood, 2008. 111(1): p. 60-70.

10. Gianelli, U., et al., Essential thrombocythemia or chronic idiopathic myelofibrosis? A single-center study based on hematopoietic bone marrow histology. Leuk Lymphoma, 2006. 47(9): p. 1774-81.

11. Thiele, J. and H.M. Kvasnicka, Chronic myeloproliferative disorders with thrombocythemia: a comparative study of two classification systems (PVSG, WHO) on 839 patients. Ann Hematol, 2003. 82(3): p. 148-52.

12. Florena, A.M., et al., Value of bone marrow biopsy in the diagnosis of essential thrombocythemia. Haematologica, 2004. 89(8): p. 911-9.

13. Brousseau, M., et al., Practical application and clinical impact of the WHO histopathological criteria on bone marrow biopsy for the diagnosis of essential thrombocythemia versus prefibrotic primary myelofibrosis. Histopathology. 56(6): p. 758-67.

14. Samuelson, S.J., C.J. Parker, and J.T. Prchal, Revised criteria for the myeloproliferative disorders: too much too soon? Blood, 2008. 111(3): p. 1741; author reply 1742. 


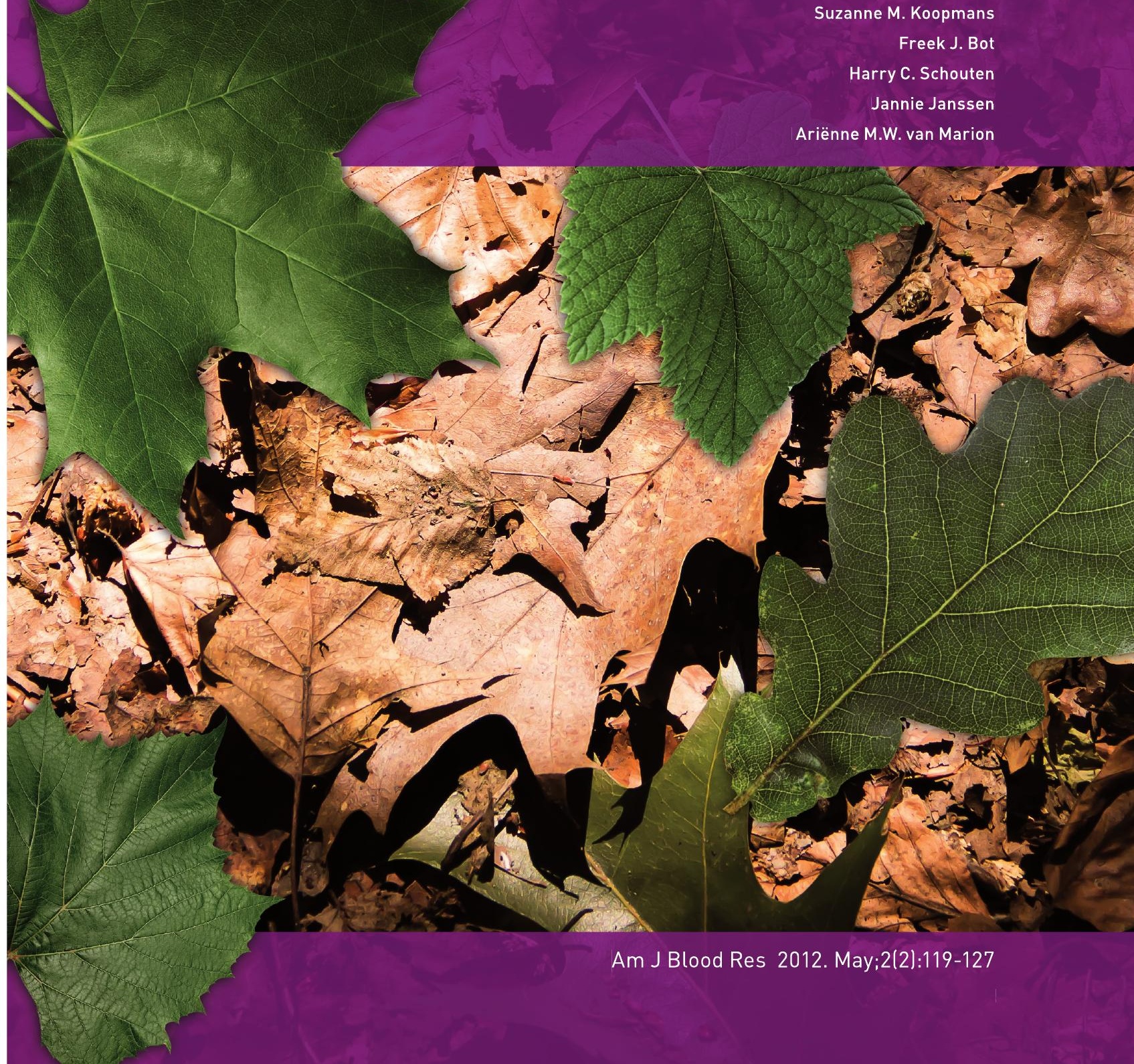




\section{ABSTRACT}

Background: In patients with myeloproliferative neoplasia (MPN) the development of fibrosis and increased vessel density correlate with poor prognosis. The JAK2 V617F mutation constitutively activates JAK2, which phosphorylates signal transducer activator of transcription (STAT), up-regulating vascular endothelial growth factor (VEGF), which might be responsible for angiogenesis in MPN. Galectins are involved in the development of fibrosis and angiogenesis and might also be involved in activation of the JAK/STAT pathway in MPN.

Methods: 106 MPN patients, 36 essential thrombocythemia (ET), 25 polycythemia vera (PV) and 45 primary myelofibrosis (PMF), were analyzed for the expression pattern of galectin-1, galectin-3, pSTAT3, pSTAT5 and MVD by immunostaining of bone marrow biopsy sections followed by automated image analysis. The JAK2 mutational status was analysed through real time PCR in blood samples.

Results: The expression of galectin-1 was significantly higher in all MPN patients compared to normal controls. Galectin-3 was expressed more in PV patients. MVD was significantly higher in all MPN patients and correlated with galectin-1 and pSTAT5 expression. pSTAT5 expression showed a trend of higher expression in patients carrying the JAK2 ${ }^{\mathrm{V} 617}$ mutation as well as in PV patients. PMF patients and all JAK2 ${ }^{\text {V617F }}$ positive patients showed a significantly higher PSTAT3 expression compared to control and ET patients.

Conclusion: The findings suggest the involvement of galectin-1 in MPN development, regardless of the subtype. Furthermore involvement of galectin-3 in PV development, pSTAT5 in that of PV and JAK2 ${ }^{V 617 F}$ positive patients and angiogenesis, as well as PSTAT3 is involved in the pathogenesis of PMF. 


\section{INTRODUCTION}

Myeloproliferative neoplasia (MPNs) are clonal bone marrow stem cell disorders, characterized by proliferation of the myeloid, erythroid and/or megakaryocytic cell lineages resulting in increased numbers of granulocytes, erythrocytes and/or platelets in the peripheral blood. The three classical Philadelphia chromosome-negative (Ph-) MPNs are polycythemia vera (PV), essential thrombocythemia (ET) and primary myelofibrosis (PMF) ${ }^{[1,2]}$. In patients with a MPN, fibrosis and increased vessel density correlate with poor prognosis ${ }^{[3,4]}$. Galectins are involved in the development of both fibrosis ${ }^{[5,6]}$ and angiogenesis ${ }^{[7]}$ in other organs, and therefore might be involved in MPN development.

Galectins mediate cell adhesion and stimulate cell migration, proliferation and apoptosis, through $\beta$-galactoside moieties on the cell surface interacting with integrins, laminin and fibronectin. Galectin-1 (gal-1) is involved in tumour angiogenesis and since increased microvessel density (MVD) has been reported in MPNs ${ }^{[8-10]}$, gal-1 might be involved in the regulation of angiogenesis in MPN. Increased galectin-3 (gal-3) expression has been shown to be involved in liver fibrosis ${ }^{[5,11]}$. Therefore, we studied the gal-1 and gal-3 expression in bone marrow trephines of Ph- MPNs.

The signal transducer and activator of transcription (STAT) proteins are activated via the JAK/ STAT pathway, by Janus Kinases (JAKs). A somatic mutation in the JAK2 gene, JAK2 ${ }^{\text {V617F }}$, has been shown to be present in $>95 \%$ of PV patients and in approximately $50 \%$ of ET and PMF patients ${ }^{[12,13]}$. The JAK2 ${ }^{V 617 F}$ mutation disrupts the inhibitory function of the pseudokinase domain in the JAK2 gene, resulting in constitutively activation of JAK2 and phosphorylation of STAT5 ${ }^{[8-10,14-16]}$. Phosphorylated STAT5 (pSTAT5) is known to be increased in PV patients ${ }^{[17,18]}$ and it was shown that activation of STAT3 induces up-regulation of vascular endothelial growth factor (VEGF) ${ }^{[19]}$. Therefore, we studied the JAK2 mutational status, PSTAT3 and PSTAT5 expression along with MVD in bone marrow trephines of patients with Ph- MPNs.

\section{MATERIALS AND METHODS}

\section{Study population}

The study was carried out on bone marrow trephines obtained from patients recorded at the Maastricht University Medical Centre, Maastricht, between January 1992 and December 2009, recorded at the Haga Hospital, The Hague, between January 2006 and December 2009 and recorded at the VieCuri Medical Centre, Venlo, between January 2005 and July 2010. The study was approved by the local institutional ethics committee. The study population consisted of 106 patients with a myeloproliferative neoplasm, with a mean age of 63.6 years at time of 
diagnosis $(S D \pm 14.7)$ ranging from 17 to 86 years. The patient population included in the study consisted of 36 ET (33.9\%), 25 PV (23.6\%), and 45 PMF (42.5\%) patients. None of the patients received therapy when the biopsy was taken. All patients were clinically and histological diagnosed according to the World Health Organization (WHO) 2008 classification $^{[20]}$ and independently reviewed by two pathologists. Of the patients 45 (42.5\%) were men and 61 (57.5\%) were women. Fifty-six patients were carriers of the JAK2 ${ }^{\text {V617F }}$ mutation (19 ET, 17 PV and 20 PMF patients), 24 patients were carriers of the JAK2 wild type (15 ET, 2 PV and 7 PMF patients) and of 26 patients the JAK2 mutational status was unknown, because of insufficient DNA to detect the JAK2 status by PCR or because the patients died prior to the availability of the $J A K 2^{\mathrm{V} 617 F}$ test (see Table 1). The patients were subdivided for the grading of myelofibrosis (mf) into $\mathrm{mf} 0 / 1$ and $\mathrm{mf} 2 / 3 ; 43$ patients belonged to the $\mathrm{mf} 0 / 1$ group (19 ET, $12 \mathrm{PV}, 12 \mathrm{PMF}$ ) of

Table 1: Clinical and laboratory findings of patients with essential thrombocthemia (ET), polycythemia vera (PV), primary myelofibrosis (PMF) and the control group.

\begin{tabular}{|c|c|c|c|c|}
\hline & $\begin{array}{c}\text { Essential } \\
\text { thrombocythemia } \\
n=36\end{array}$ & $\begin{array}{l}\text { Polycythemia } \\
\text { vera } \\
n=25\end{array}$ & $\begin{array}{c}\text { Primary } \\
\text { myelofibrosis } \\
n=45\end{array}$ & $\begin{array}{c}\text { Control bone } \\
\text { marrow } \\
n=36\end{array}$ \\
\hline Males / females & $11 / 25$ & $8 / 17$ & $26 / 19$ & $23 / 13$ \\
\hline $\begin{array}{l}\text { Age, y, } \\
\text { mean (SD) }\end{array}$ & $\begin{array}{c}59 \\
(17,70)\end{array}$ & $\begin{array}{c}65 \\
(13,56)\end{array}$ & $\begin{array}{c}67 \\
(10,73)\end{array}$ & $\begin{array}{c}56 \\
(14,33)\end{array}$ \\
\hline $\begin{array}{l}\text { JAK2 wild type / } \\
\text { JAK2 mutation / } \\
\text { JAK2 unknown }\end{array}$ & $\begin{array}{c}15 \\
19 \\
2\end{array}$ & $\begin{array}{c}2 \\
17 \\
6\end{array}$ & $\begin{array}{c}7 \\
20 \\
18\end{array}$ & $\begin{array}{c}36 \\
0 \\
0\end{array}$ \\
\hline $\begin{array}{l}\text { White blood cell } \\
\text { count* } 10^{9} / \mathrm{L} \text {, } \\
\text { mean (SD) }\end{array}$ & $\begin{array}{l}9,37 \\
(2,89)\end{array}$ & $\begin{array}{c}16,08 \\
(12,22)\end{array}$ & $\begin{array}{c}11,89 \\
(11,38)\end{array}$ & $\begin{array}{l}8,16 \\
(4,44)\end{array}$ \\
\hline Minimum-maximum & $4,4-18,30$ & $5,70-62,00$ & $0,90-70,60$ & $2,80-23,80$ \\
\hline $\begin{array}{l}\text { Haemoglobin, } \mathrm{mmol} / \mathrm{L} \text {, } \\
\text { mean (SD) }\end{array}$ & $\begin{array}{c}8,53 \\
(1,26)\end{array}$ & $\begin{array}{l}9,89 \\
(1,81)\end{array}$ & $\begin{array}{c}7,09 \\
(1,58)\end{array}$ & $\begin{array}{l}8,16 \\
(1,10)\end{array}$ \\
\hline Minimum-maximum & $6,10-12,00$ & $6,70-13,50$ & $3,30-10,60$ & $6,10-11,10$ \\
\hline $\begin{array}{l}\text { Haematocrit L/L, } \\
\text { mean (SD) }\end{array}$ & $\begin{array}{l}0,43 \\
(0,06)\end{array}$ & $\begin{array}{l}0,52 \\
(0,09)\end{array}$ & $\begin{array}{c}0,35 \\
(0,08)\end{array}$ & $\begin{array}{l}0,39 \\
(0,05)\end{array}$ \\
\hline Minimum-maximum & $0,29-0,61$ & $0,37-0,68$ & $0,15-0,50$ & $0,30-0,52$ \\
\hline $\begin{array}{l}\text { Thrombocytes*10\%/L, } \\
\text { mean (SD) }\end{array}$ & $\begin{array}{l}929 \\
(346)\end{array}$ & $\begin{array}{c}662 \\
(316)\end{array}$ & $\begin{array}{c}564 \\
(532)\end{array}$ & $\begin{array}{l}263 \\
(137)\end{array}$ \\
\hline Minimum-maximum & $327-1862$ & $112-1371$ & $15-2644$ & 49-585 \\
\hline
\end{tabular}


which 24 were JAK2 ${ }^{\mathrm{V} 617 F}$ positive and 11 carried the JAK2 wild type gene and 61 belonged to the $\mathrm{mf} 2 / 3$ group (17 ET, $12 \mathrm{PV}, 32 \mathrm{PMF}$ ) of which 31 were JAK2 ${ }^{\mathrm{V} 617 F}$ positive and 13 carried the JAK2 wild type gene. The control group consisted of 36 morphologically normal negative staging biopsies from patients with non-Hodgkin lymphoma and Hodgkin lymphoma with a mean age of 55.8 years.

\section{Immunohistochemistry}

The bone marrow biopsy specimens were decalcified using the EDTA decalcification for four hours, followed by standard tissue processing and paraffin embedding. From the paraffinembedded blocks $3 \mu \mathrm{m}$ sections were cut for immunohistochemical staining and mounted on starfrost slides (Knittel Gläser, Germany). All the antibodies were tested for specificity on positive and negative tumour control slides and also individually tested on decalcified control bone marrow biopsies, resulting in a variation of immunohistochemical procedures, optimised for all individual antibodies.

Antihuman galectin-1 (R\&D systems, Minneapolis, MN) was used with a dilution of 1:500 and antihuman galectin-3 (R\&D systems, Minneapolis, MN) with a dilution of 1:50. After deparaffinization and blocking of endogenous peroxidase activity $\left(0.3 \% \mathrm{H}_{2} \mathrm{O}_{2}\right.$ in methanol) antigen retrieval was performed by boiling in citric acid $(\mathrm{pH}$ 6.0) for 10 minutes in a water bath of $100^{\circ} \mathrm{C}$. After blocking with $5 \%$ bovine serum albumin/phosphate buffered saline (BSA/ PBS), primary antibodies were applied in 0.5\% BSA/PBS. The slides were then incubated with a biotin-labelled secondary antibody Igal-1: polyclonal swine anti-rabbit, Dako (Glostrup, Denmark) and gal-3: rabbit anti-goat, Dako (Glostrup, Denmark)) with a dilution of $1: 200$ and $1: 500$ respectively for 30 minutes. Staining was performed with the StrepABComplex/ HRP kit (Dako, Glostrup, Denmark) according to the manufacturer's instructions. After developing the colour with freshly made diaminobenzidine solution (Dako, Glostrup, Denmark), slides were counterstained with haematoxylin (Merck, Whitehouse Station, NJ), dehydrated and mounted in Entellan (Merck).

Immunohistochemical staining of pSTAT3 and pSTAT5 was carried out using the antihuman rabbit monoclonal antibody pSTAT3 (Tyr705) and pSTAT5 (Tyr694) with a dilution of 1:50 and 1:200 respectively (Cell signaling Technology, Danvers, MA). After deparaffinization and antigen retrieval by boiling for 20 minutes in $1 \mathrm{mM}$ Tris EDTA pH 8.0 in a warm water bath, endogenous peroxidase activity was blocked in $3 \% \mathrm{H}_{2} \mathrm{O}_{2}$ in methanol. After blocking with the blocking solution (Tris Buffered Saline Tween (TBST) with 5\% horse serum), the primary antibodies were applied in TBST with $5 \%$ horse serum (pSTAT3) and TBST with 1\% BSA (pSTAT5) overnight. The slides were then incubated with powervision (Immunologic, Duiven, The Netherlands) for 40 minutes. Development of the colour and counterstaining was performed as described above. 
The 142 trephines (MPN patients plus control patients) were immunohistochemically analysed using an automated immunostainer (Dako autostainer Link 48) with CD34 (clone QBend 10, Dako). CD34 was incubated for 20 minutes at room temperature. The reaction was revealed by means of the Dako Envision Flex Kit (Dako) according to the manufacturer's instructions.

\section{Quantification of staining}

Gal-1, gal-3, pSTAT3 and pSTAT5 staining (see Figure 1) was quantified using an image processing and analysis system (Leica, Cambridge, UK) linked to a Leica DML3000 light microscope (Leica Quantimet, Germany). QWin (Leica's Windows-based image analysis toolkit-Leica, Cambridge, UK) was used for postprocessing. The surface area of galectin present was measured separately in cell nuclei and in stroma. All measurements were conducted with a magnification factor of 40 , in at least three to at most five complete hot spot bone marrow fields per slide, to measure total tissue, total cytoplasmic area positive and negative staining (gal-1 and gal-3), total nuclei positive (pSTAT3 or pSTAT5) and total nuclei count. The amount of positivity was calculated as the percentage of the total tissue area (gal-1 and gal-3)

Figure 1: Examples of galectin-1, galectin-3, pSTAT3 and pSTAT5 staining.

The brown colour represents the gal-1 staining in A and B, gal-3 staining in C, pSTAT3 staining in D and pSTAT5 staining in E and F. A. Galectin-1 (630x) B. Galectin-1 (1000x) C. Galectin-3 (630x) D. pSTAT3 (630x) E. pSTAT5 (630x) F. pSTAT5 (1000x).
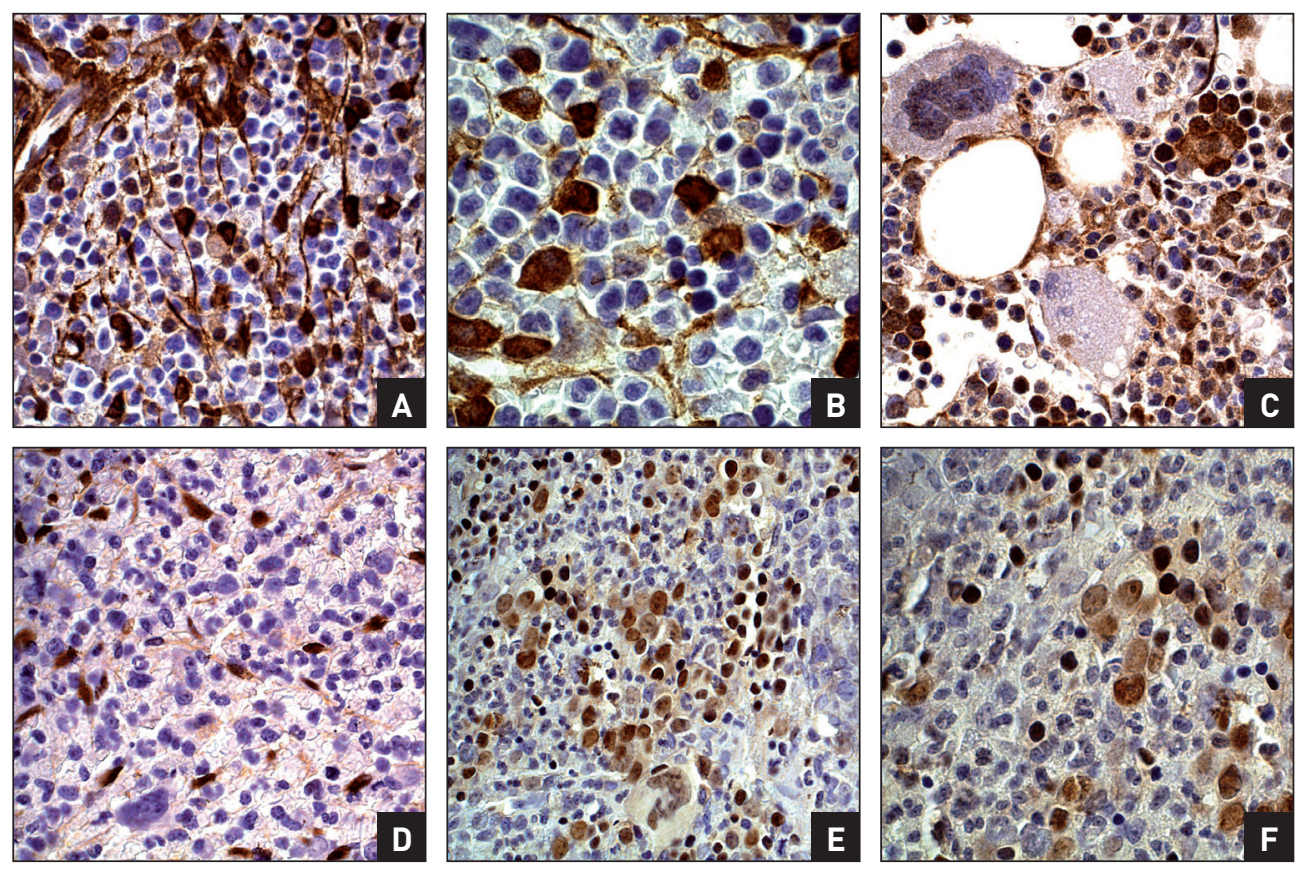
or the percentage of positive nuclear pixels related to the total number of nuclear pixels (pSTAT3 and pSTAT5). MVD was assessed by counting the number of CD34 positive capillary-, arteriolar- or sinuslumen in five $1 \mathrm{~mm}^{2}$ fields at 100x magnification, calculating the mean over these five fields. The grading of fibrosis was done according to the European consensus on grading of bone marrow fibrosis ${ }^{[21]}$.

To validate the data obtained at the molecular level, we tried to isolate DNA from bone marrow biopsies. However, the quality of the DNA was very poor and the DNA was too fragmented to be used.

\section{Statistical analysis}

The data were statistically evaluated using the SPSS 15 statistical package, analyzed descriptively (descriptives, explore and crosstabs). Statistical comparison was performed by Mann-Whitney $U$-test when comparing medians. Differences were considered significant when $p$-value was less then 0.05. Pearson's test was performed for correlating the expression of gal-1 with MVD, gal-3 with MVD, pSTAT3 with MVD and pSTAT5 with MVD.

For the analysis of pSTAT5, bone marrow of the Haga hospital, The Hague, was withdrawn, due to inappropriate staining of the bone marrow. Only 30 ET patients, 16 PV and 34 PMF patients and a total of 20 control bone marrows were available for pSTAT5 analysis.

In some cases bone marrow tissue was lost during the pre-treatment of the slides; for gal-1 we report 1 missing value, for PSTAT5 6, and for MVD 5 missing values. For the grading of myelofibrosis we report 2 missing values. 
Table 2: Percentage of gal-1, gal-3, pSTAT3 and pSTAT5 in essential thrombocythemia (ET), polycythemia vera (PV), primary melofibrosis (PMF), all MPN patients and control patients.

\begin{tabular}{|c|c|c|c|c|c|}
\hline & $\underset{n=36}{E T}$ & $\begin{array}{c}P V \\
n=25\end{array}$ & $\begin{array}{l}\text { PMF } \\
n=45\end{array}$ & $\begin{array}{c}\text { All MPN } \\
\text { patients } \\
n=106\end{array}$ & $\begin{array}{c}\text { Control } \\
\text { patients } \\
n=36\end{array}$ \\
\hline Galectin-1, \%*, mean (SD) & 7.80 (4.37) & $8.15(4.50)$ & 7.70 (3.35) & 7.84 (3.97) & 6.25 (2.65) \\
\hline $\begin{array}{l}\text { Minimum-maximum } \\
\text { (CI) }\end{array}$ & $\begin{array}{cc}1.12 & -20.32 \\
(5,90 & -9.49)\end{array}$ & $\begin{array}{cc}0.49 & -12.79 \\
{[5.69} & -10.09]\end{array}$ & $\begin{array}{l}3.65-14.56 \\
(6.27-8.95)\end{array}$ & $\begin{array}{cc}0.49 & -20.32 \\
(6.76 & -8.65)\end{array}$ & $\begin{array}{l}3.22-15.60 \\
(4.23-8.57)\end{array}$ \\
\hline $\begin{array}{l}\text { Galectin-3, \%*, mean } \\
\text { (SD) }\end{array}$ & $7.24(4.82)$ & $10.23(5.01)$ & $7.72(5.90)$ & 8.15 (5.43) & $8.58(4.51)$ \\
\hline $\begin{array}{l}\text { Minimum-maximum } \\
\text { (CI) }\end{array}$ & $\begin{array}{r}0.64-18.69 \\
(5.76-9,52)\end{array}$ & $\begin{array}{c}0.20-19.51 \\
(6.53-12.75)\end{array}$ & $\begin{array}{cc}0.56 & -23.50 \\
(6.28 & -11.43)\end{array}$ & $\begin{array}{cc}0.20 & -23.50 \\
(7.19 & -9.89)\end{array}$ & 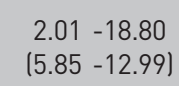 \\
\hline pSTAT3, \%\#, mean (SD) & $4.18(1.96)$ & $5.19(4.21)$ & 5.52 (3.29) & 4.99 (3.20) & $4.21(2.28)$ \\
\hline $\begin{array}{l}\text { Minimum-maximum } \\
\text { (CI) }\end{array}$ & $\begin{array}{c}1.12-8.91 \\
(3.17-4.94)\end{array}$ & $\begin{array}{r}1.02-16.53 \\
(2.77-7.63)\end{array}$ & $\begin{array}{c}0.84-13.68 \\
(3.98-6.42)\end{array}$ & $\begin{aligned} 0.84 & -16.53 \\
(3.99 & -5.49)\end{aligned}$ & $\begin{array}{cc}0.96 & -7.92 \\
(2.67 & -5.17)\end{array}$ \\
\hline pSTAT5, \%\#, mean (SD) & $2.91(2.18)$ & $4.72(3.58)$ & $3.31(2.60)$ & $3.46(2.74)$ & $3.62(2.46)$ \\
\hline $\begin{array}{l}\text { Minimum-maximum } \\
\text { (CI) }\end{array}$ & $\begin{array}{rr}0.26 & -7.40 \\
12.06 & -3.75\end{array}$ & $\begin{array}{r}0,40-11.77 \\
(2.82-6.63)\end{array}$ & $\begin{array}{r}0.00-13.71 \\
(2.41-4.22)\end{array}$ & 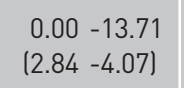 & $\begin{array}{c}1.18-9.29 \\
(2.08-4.65)\end{array}$ \\
\hline MVD, $1 \mathrm{~mm}^{2}$, mean (SD) & $37.72(22.18)$ & $47.55(27.45)$ & $58.47(31.56)$ & $48.79(28.92)$ & 27.95 (11.25) \\
\hline $\begin{array}{l}\text { Minimum-maximum } \\
\text { (CI) }\end{array}$ & $\begin{array}{c}3.40-89,60 \\
(29.70-49.07)\end{array}$ & $\begin{array}{rr}5.80 & 111.20 \\
(35.77 & -70.80)\end{array}$ & $\begin{array}{r}12.80-122.40 \\
(50.30-76.39)\end{array}$ & $\begin{array}{rr}3.40 & -122.40 \\
(44.16 & -59.03)\end{array}$ & $\begin{aligned} 5.60 & -57.80 \\
(17.94 & -34.27)\end{aligned}$ \\
\hline
\end{tabular}

* calculated as percentage positive area of total tissue area

\# calculated as percentage positive nuclei of total nuclei count 


\section{RESULTS}

The results of all staining percentages are summarized in Table 2 and 3. Qualitative microscopic evaluation of gal-1 staining showed its expression mainly in the immature myeloid cell component. A weak expression of gal-1 was seen in the cytoplasm of the megakaryocytes, no expression of gal-1 was seen in the erythroid cell line. Gal-1 was expressed significantly more in bone marrow of PMF patients compared to the control slides ( $p=0.036$ ). The mean percentage of gal- 1 for all MPN patients together was $7.8 \%$ and $6.3 \%$ for the control patients $(p=0.027)$. The expression between gal- 1 and MVD was significantly correlated ( $p=0.007)$.

Gal-3 was present in immature and mature myeloid cells and was only weakly expressed in megakaryocytes, endothelial cells and erythropoietic cells. Statistical analysis of gal-3 revealed a significant difference between $\mathrm{PV}$ and ET patients $(p=0.019)$ and between $\mathrm{PV}$ and PMF ( $p=0.044)$ patients, with higher gal-3 expression in PV patients. There was no significant correlation between gal-3 and MVD and no significant difference between patients with different JAK2 mutational status.

pSTAT3 was localized in immature and mature myeloid cells and in endothelial cells. In the evaluated bone marrow biopsy trephines, the percentage of pSTAT3 was higher in JAK2 ${ }^{\text {V617F }}$ positive patients compared to patients with wild type JAK2 $(p=0.018)$. There was also a significant correlation between $p S T A T 3$ and MVD $p=0.000$ ). pSTAT5 was expressed in immature myeloid cells, the nuclei of adipocytes, some endothelial cells and in the nuclei of megakaryocytes and partly a weak expression in the cytoplasm of megakaryocytes. pSTAT5 was significantly correlated with the MVD ( $p=0.020)$. No statistically significant difference but a trend was reached between patients carrying the JAK2 ${ }^{\mathrm{V} 617 F}$ mutation and patients without the mutation as well as in PV patients compared to ET and PMF patients.

In the total MPN group the mean MVD was significantly higher compared to the control group $(p=0.000)$ and the MVD was significantly higher expressed in PV $(p=0.006)$ and PMF $(p=0.000)$ patients compared to the control group. ET patients compared to PMF patients showed also a statistically significant difference with a higher MVD expression in PMF patients ( $p=0.003$ ). PMF patients showed higher MVD (58.5 vessels/mm2) than ET (37.7 vessels/mm2) and PV patients (47.6 vessels $/ \mathrm{mm} 2$ ). Comparing the JAK2 ${ }^{\text {V617F }}$ positive patients to the JAK2 ${ }^{\text {V617F }}$ negative patients the MVD was not significantly different.

Concerning the myelofibrosis grading and the stainings we report a statistically significant higher gal- 1 ( $p=0.013$ ) and gal-3 ( $p=0.012$ ) expression in the $\mathrm{mf} 0 / 1$ group compared to the $\mathrm{mf}$ $2 / 3$ group. For MVD there was a higher expression of MVD in the $\mathrm{mf} 2 / 3$ group $(p=0.001$ ) compared to the $\mathrm{mf} 0 / 1$ group and also the pearson correlation showed a significant correlation of MVD with the grading of myelofibrosis ( $p=0.000)$. 
Table 3: Percentage of gal-1, gal-3, pSTAT3 and pSTAT5 in JAK2 positive and JAK2 negative patients

\begin{tabular}{|c|c|c|}
\hline & $\begin{array}{c}\text { JAK2 positive } \\
n=56\end{array}$ & $\begin{array}{c}\underset{n=24}{\text { Wild type JAK2 }} \\
n=2\end{array}$ \\
\hline $\begin{array}{l}\text { Galectin-1, \%*, } \\
\text { mean (SD) }\end{array}$ & $\begin{array}{c}8.50 \\
(4.04)\end{array}$ & $\begin{array}{c}7.36 \\
(3.58)\end{array}$ \\
\hline $\begin{array}{l}\text { Minimum-maximum } \\
\text { (CI) }\end{array}$ & $\begin{array}{l}1.12-20.32 \\
(7.41-9.59)\end{array}$ & $\begin{array}{l}1.48-14.56 \\
(5.82-8.91)\end{array}$ \\
\hline $\begin{array}{l}\text { Galectin-3. \%* } \\
\text { mean (SD) }\end{array}$ & $\begin{array}{c}8.93 \\
(5.34)\end{array}$ & $\begin{array}{c}7.04 \\
(5.18)\end{array}$ \\
\hline $\begin{array}{l}\text { Minimum-maximum } \\
\text { (CI) }\end{array}$ & $\begin{array}{c}0.56-23.50 \\
(7.49-10.37)\end{array}$ & $\begin{array}{l}0.55-18.69 \\
(4.80-9.28)\end{array}$ \\
\hline $\begin{array}{l}\text { pSTAT3. \%\#. } \\
\text { mean (SD) }\end{array}$ & $\begin{array}{c}5.53 \\
(2.80)\end{array}$ & $\begin{array}{c}4.18 \\
(1.97)\end{array}$ \\
\hline $\begin{array}{l}\text { Minimum-maximum } \\
\text { (CI) }\end{array}$ & $\begin{array}{l}1.02-14.67 \\
(4.77-6.29)\end{array}$ & $\begin{array}{c}1.12-8.91 \\
(3.33-5.03)\end{array}$ \\
\hline $\begin{array}{l}\text { pSTAT5. \%\#. } \\
\text { mean (SD) }\end{array}$ & $\begin{array}{c}4.22 \\
(3.48)\end{array}$ & $\begin{array}{c}3.04 \\
(1.60)\end{array}$ \\
\hline $\begin{array}{l}\text { Minimum-maximum } \\
\text { (CI) }\end{array}$ & $\begin{array}{l}0.26-13.71 \\
(3.02-5.41)\end{array}$ & $\begin{array}{c}0.90-6.47 \\
(2.27-3.81)\end{array}$ \\
\hline $\begin{array}{l}\text { MVD. } 1 \mathrm{~mm} 2 . \text { mean } \\
\text { (SD) }\end{array}$ & $\begin{array}{c}52.77 \\
(30.58)\end{array}$ & $\begin{array}{c}49.01 \\
(25.98)\end{array}$ \\
\hline $\begin{array}{l}\text { Minimum-maximum } \\
\text { (CI) }\end{array}$ & $\begin{array}{c}3.60-122.40 \\
(44.72-63.18)\end{array}$ & $\begin{array}{l}17.20-111.20 \\
(40.64-65.24)\end{array}$ \\
\hline
\end{tabular}

* calculated as percentage positive area of total tissue area

\# calculated as percentage positive nuclei of total nuclei count 


\section{DISCUSSION}

In this study, the expression of gal-1, gal-3, pSTAT3 and pSTAT5 along with the MVD in bone marrow cells was immunohistochemically measured in ET, PV, PMF and control bone marrows.

Gal-1 is known to be involved in tumour angiogenesis ${ }^{[7]}$. The higher expression of gal-1 and MVD in the total group of MPN patients in our study together with a significant correlation between gal-1 and MVD, suggests a role of gal-1 in the increased angiogenesis in MPN patients. These results assign a possible target for the angiogenesis inhibitor anginex, as gal1 was identified as receptor for anginex. Anginex blocks the adhesion and migration of angiogenicallyactivated endothelial cells, leading to apoptosis and inhibition of angiogenesis ${ }^{[22]}$. In gal-1-null mice treatment with anginex did not inhibit tumour growth in contrast to the wild type mice where tumour growth and vessel density was significantly inhibited with anginex treatment ${ }^{[7]}$.

Increased expression of gal-3 has been associated with liver fibrosis secondary to diverse types of injury ${ }^{[11]}$. However, in the $\mathrm{mf} 0 / 1$ group we saw a higher gal-3 expression compared to the $\mathrm{mf} 2 / 3$ group. Also we saw no significant correlation between gal-3 and MVD. These findings contradict the relation between increasing fibrosis, MVD and gal-3 expression in MPN trephines. On the other hand we were able to show higher gal-3 expression in PV patients. Recently, it was also demonstrated that gal-3 is predominantly expressed in Chronic Myeloid Leukemia (CML) cells, where gal-3 expression support the molecular signalling pathways for maintaining $C M L$ in the bone marrow and resistance to therapy ${ }^{[23,24]}$. Therefore there are indications that gal-3 might play a role in MPN pathogenesis.

Constitutive activation of STAT proteins is present in a variety of haematological disorders ${ }^{[25-29]}$. STAT3 activation has been reported in PV and ET and low pSTAT3 levels in PMF patients ${ }^{[17,30]}$. However, our study does not confirm these results, possibly due to a relative high amount of PMF patients and lower amounts of PV and ET patients.

Activated STAT3 has an important role in the regulation of megakaryopoiesis and thrombopoiesis in vivo, via activation of $\mathrm{Bcl}-\mathrm{xL}$ inhibiting apoptosis of megakaryocytes ${ }^{[31]}$. The bone marrow of PMF patients is characterized by a proliferation of the megakaryocytic cell line. The megakaryocytes often demonstrate dense clustering with cloud like nucleus[20]. The increased megakaryocytes with deviated forms in the bone marrow of PMF patients might be due to the decreased megakaryocyte apoptosis as result of increased STAT3 activation in PMF patients. The higher pSTAT3 expression in JAK2 ${ }^{\mathrm{V} 617 \mathrm{~F}}$ positive patients indicates an increased STAT3 activation generated by the presence of the JAK2 ${ }^{\mathrm{V} 17 F}$ mutation. In diverse cancer types it was shown that constitutive activation of STAT3 induces vascular endothelial growth factor (VEGF) expression ${ }^{[19]}$. In our study we show a correlation between 
pSTAT3 and MVD, indicating that the increased MVD seen in MPN patients, especially in PMF patients, might be induced by the constitutive activation of STAT3 resulting in increased expression of VEGF.

Our finding of higher pSTAT5 expression in PV and JAK2 ${ }^{\text {V617F }}$ positive patients is in line with earlier published data ${ }^{[14,17,32,33]}$. This indicates that the presence of the JAK2 ${ }^{\mathrm{V} 617 F}$ mutation generates increased levels of pSTAT5. However, in our study the pSTAT5 expression did not reach statistical significant difference but only showed a trend between patients carrying the $J A K 2^{V 617 F}$ mutation and patients without the mutation as well as in PV patients compared to ET and PMF patients. This might be due to the high number of patients with an unknown JAK2 status and also to the small PV patient population. The correlation between pSTAT5 and MVD might suggest other pathways involved in the increased MVD seen in MPN patients. pSTAT5 can interact with p85, a regulatory subunit of PI3K/Akt pathway, and might increase VEGF via the PI3K/Akt and mammalian target of rapamycine (mTor) pathway as was already shown in chronic myeloid leukaemia (CML) ${ }^{[34-36]}$.

In line with other studies ${ }^{[37,38]}$, we found the bone marrow MVD in the total MPN group and in PV and PMF patients to be significantly higher compared to the control group. The increased MVD reflects increased angiogenic activity which might be induced by hypoxia, via hypoxiainducible factor (Hif) and VEGF, or by normoxia, directly via VEGF.

Regarding the MVD and fibrosis in MPN patients, Boveri et al. ${ }^{[39]}$ found a higher MVD along with a higher grading of fibrosis, which is line with our study. Other studies showed higher MVD in PMF, post-ET myelofibrosis and post-PV myelofibrosis patients compared to ET and $P V$ patients indicating that angiogenesis is primarily involved in later stages of the disease ${ }^{[38-41]}$.

In conclusion, the characteristic megakaryopoietic abnormalities and also the higher MVD expression in PMF trephines can be explained by a higher pSTAT3 expression in PMF patients. Also gal-1 expression is correlated with the MVD with anginex as potential new therapy for MPN patients. pSTAT5 expression showed a trend of higher expression in PV and JAK2 ${ }^{\text {V617F }}$ positive patients, possible induced by the JAK2 $2^{\mathrm{V} 617 \mathrm{~F}}$ mutation and also gal-3 expression seems correlated with PV. Further, the increased MVD expression in MPN patients with higher myelofibrosis grading suggests the important role of angiogenesis in the development of myelofibrosis.

Based upon these data we support the concept that the microenvironment plays an important role in haematological malignancies ${ }^{[42,43]}$. Interactions between stroma and haematopoietic cells in MPNs constitute possible targets for therapy. 


\section{REFERENCES}

1. Campbell, P.J. and Green, A.R., The myeloproliferative disorders. N Engl J Med, 2006. 355(23): p. 2452-66.

2. Murray, J., Myeloproliferative disorders. Clin Med, 2005. 5(4): p. 328-32.

3. Ponzoni, M., Savage, D.G., Ferreri, A.J., Pruneri, G., Viale, G., Servida, P., Bertolini, F., Orazi, A., Chronic idiopathic myelofibrosis: independent prognostic importance of bone marrow microvascular density evaluated by CD105 (endoglin) immunostaining. Mod Pathol, 2004. 17(12): p. 1513-20.

4. Vener, C., Fracchiolla, N.S., Gianelli, U., Calori, R., Radaelli, F., Iurlo, A., Caberlon, S., Gerli, G., Boiocchi, L., Deliliers, G.L., Prognostic implications of the European consensus for grading of bone marrow fibrosis in chronic idiopathic myelofibrosis. Blood, 2008. 111(4): p. 1862-5.

5. Hsu, D.K., Dowling, C.A., Jeng, K.C., Chen, J.T., Yang, R.Y., Liu, F.T., Galectin-3 expression is induced in cirrhotic liver and hepatocellular carcinoma. Int J Cancer, 1999. 81(4): p. 519-26.

6. Nishi, Y., Sano, H., Kawashima, T., Okada, T., Kuroda, T., Kikkawa, K., Kawashima, S., Tanabe, M., Goto, T., Matsuzawa, Y., Matsumura, R., Tomioka, H., Liu, F.T., Shirai, K., Role of galectin-3 in human pulmonary fibrosis. Allergol Int, 2007. 56(1): p. 57-65.

7. Thijssen, V.L., Postel, R., Brandwijk, R.J., Dings, R.P., Nesmelova, I., Satijn, S., Verhofstad, N., Nakabeppu, Y., Baum, L.G., Bakkers, J., Mayo, K.H., Poirier, F., Griffioen, A.W., Galectin-1 is essential in tumor angiogenesis and is a target for antiangiogenesis therapy. Proc Natl Acad Sci U S A, 2006. 103(43): p. 15975-80.

8. Baxter, E.J., Scott, L.M., Campbell, P.J., East, C., Fourouclas, N., Swanton, S., Vassiliou, G.S., Bench, A.J., Boyd, E.M., Curtin, N., Scott, M.A., Erber, W.N., Green, A.R., Acquired mutation of the tyrosine kinase JAK2 in human myeloproliferative disorders. Lancet, 2005. 365(9464): p. 1054-61.

9. Kralovics, R., Passamonti, F., Buser, A.S., Teo, S.S., Tiedt, R., Passweg, J.R., Tichelli, A., Cazzola, M., Skoda, R.C., A gain-of-function mutation of JAK2 in myeloproliferative disorders. N Engl J Med, 2005. 352(17): p. 1779-90.

10. Levy, D.E. and Darnell, J.E. Jr., Stats: transcriptional control and biological impact. Nat Rev Mol Cell Biol, 2002. 3(9): p. 651-62.

11. Henderson, N.C., Mackinnon, A.C., Farnworth, S.L., Poirier, F., Russo, F.P., Iredale, J.P., Haslett, C., Simpson, K.J., Sethi, T., Galectin-3 regulates myofibroblast activation and hepatic fibrosis. Proc Natl Acad Sci U S A, 2006. 103(13): p. 5060-5.

12. Tiedt, R., Hao-Shen, H., Sobas, M.A., Looser, R., Dirnhofer, S., Schwaller, J., Skoda, R.C., Ratio of mutant JAK2-V617F to wild-type JAK2 determines the MPD phenotypes in transgenic mice. Blood, 2008. 111(8): p. $3931-40$

13. Vannucchi, A.M., Antonioli, E., Guglielmelli, P., Pardanani, A., Tefferi, A., Clinical correlates of JAK2 ${ }^{\mathrm{V} 617 F}$ presence or allele burden in myeloproliferative neoplasms: a critical reappraisal. Leukemia, 2008. 22(7): p. 1299-307.

14. James, C., Ugo, V., Le Couédic, J.P., Staerk, J., Delhommeau, F., Lacout, C., Garçon, L., Raslova, H., Berger, R., Bennaceur-Griscelli, A., Villeval, J.L., Constantinescu, S.N., Casadevall, N., Vainchenker, W., A unique clonal JAK2 mutation leading to constitutive signalling causes polycythaemia vera. Nature, 2005. 434(7037): p. 1144-8. 
15. Levine, R.L., Loriaux, M., Huntly, B.J., Loh, M.L., Beran, M., Stoffregen, E., Berger, R., Clark, J.J., Willis, S.G., Nguyen, K.T., Flores, N.J., Estey, E., Gattermann, N., Armstrong, S., Look, A.T., Griffin, J.D., Bernard, O.A., Heinrich, M.C., Gilliland, D.G., Druker, B., Deininger, M.W., Activating mutation in the tyrosine kinase JAK2 in polycythemia vera, essential thrombocythemia, and myeloid metaplasia with myelofibrosis. Cancer Cell, 2005. 7(4): p. 387-97.

16. O'Shea, J.J., Gadina, M., and Schreiber, R.D., Cytokine signaling in 2002: new surprises in the Jak/Stat pathway. Cell, 2002. 109 Suppl: p. S121-31.

17. Teofili, L., Martini, M., Cenci, T., Petrucci, G., Torti, L., Storti, S., Guidi, F., Leone, G., Larocca, L.M., Different STAT-3 and STAT-5 phosphorylation discriminates among Ph-negative chronic myeloproliferative diseases and is independent of the V617F JAK-2 mutation. Blood, 2007. 110(1): p. 354-9.

18. Zhao, R., Xing, S., Li, Z., Fu, X., Li, Q., Krantz, S.B., Zhao, Z.J., Identification of an acquired JAK2 mutation in polycythemia vera. J Biol Chem, 2005. 280(24): p. 22788-92.

19. Niu, G., Wright, K.L., Huang, M., Song, L., Haura, E., Turkson, J., Zhang, S., Wang, T., Sinibaldi, D., Coppola, D., Heller, R., Ellis, L.M., Karras, J., Bromberg, J., Pardoll, D., Jove, R., Yu, H., Constitutive Stat3 activity up-regulates VEGF expression and tumor angiogenesis. Oncogene, 2002. 21(13): p. 2000-8.

20. Swerdlow, S.H., S.H., Campo, E., Harris, N.L., Jaffe, E.S., Pileri, S.A., Stein, H., Thiele, J., Vardiman, J.W., WHO Classification of Tumours of Haematopoietic and Lymphoid Tissues, Fourth Edition. WHO Classification of Tumours, Volume 2. IARC WHO Classification of Tumours, No 2. 2008.

21. Thiele, J., Kvasnicka, H.M., Facchetti, F., Franco, V., van der Walt, J., Orazi, A., European consensus on grading bone marrow fibrosis and assessment of cellularity. Haematologica, 2005. 90(8): p. 1128-32.

22. Griffioen, A.W., van der Schaft, D.W., Barendsz-Janson, A.F., Cox, A., Struijker Boudier, H.A., Hillen, H.F., Mayo, K.H., Anginex, a designed peptide that inhibits angiogenesis. Biochem J, 2001. 354(Pt 2): p. 233-42.

23. Yamamoto-Sugitani, Kuroda, J., Ashihara, E., Nagoshi, H., Kobayashi, T., Matsumoto, Y., Sasaki, N., Shimura, Y., Kiyota, M., Nakayama, R., Akaji, K., Taki, T., Uoshima, N., Kobayashi, Y., Horiike, S., Maekawa, T., Taniwaki, M., et al., Galectin-3 (Gal-3) induced by leukemia microenvironment promotes drug resistance and bone marrow lodgment in chronic myelogenous leukemia. Proc Natl Acad Sci U SA, 2011. 108(42): p. 17468-73.

24. Cheng, Y.L., Huang, W.C., Chen, C.L., Tsai, C.C., Wang, C.Y., Chiu, W.H., Chen, Y.L., Lin, Y.S., Chang, C.F., Lin, C.F., Increased galectin-3 facilitates leukemia cell survival from apoptotic stimuli. Biochem Biophys Res Commun, 2011. 412(2): p. 334-40.

25. Carlesso, N., Frank, D.A, and Griffin, J.D., Tyrosyl phosphorylation and DNA binding activity of signal transducers and activators of transcription (STAT) proteins in hematopoietic cell lines transformed by Bcr/Abl. J Exp Med, 1996. 183(3): p. 811-20.

26. Gouilleux-Gruart, V., Gouilleux, F., Desaint, C., Claisse, J.F., Capiod, J.C., Delobel, J., Weber-Nordt, R., Dusanter-Fourt, I., Dreyfus, F., Groner, B., Prin, L., STAT-related transcription factors are constitutively activated in peripheral blood cells from acute leukemia patients. Blood, 1996. 87(5): p. 1692-7.

27. Weber-Nordt, R.M., Egen, C., Wehinger, J., Ludwig, W., Gouilleux-Gruart, V., Mertelsmann, R., Finke, J., Constitutive activation of STAT proteins in primary lymphoid and myeloid leukemia cells and in EpsteinBarr virus (EBV)-related lymphoma cell lines. Blood, 1996. 88(3): p. 809-16.

28. Xia, Z., Baer, M.R., Block, A.W., Baumann, H., Wetzler, M., Expression of signal transducers and activators of transcription proteins in acute myeloid leukemia blasts. Cancer Res, 1998. 58(14): p. 3173-80.

29. Frank, D.A., STAT signaling in the pathogenesis and treatment of cancer. Mol Med, 1999. 5(7): p. $432-56$. 
30. Roder, S., Steimle, C., Meinhardt, G., Pahl, HL., STAT3 is constitutively active in some patients with Polycythemia rubra vera. Exp Hematol, 2001. 29(6): p. 694-702.

31. Kirito, K., Osawa, M., Morita, H., Shimizu, R., Yamamoto, M., Oda, A., Fujita, H., Tanaka, M., Nakajima, K., Miura, Y., Ozawa, K., Komatsu, N., A functional role of Stat3 in in vivo megakaryopoiesis. Blood, 2002. 99(9): p. 3220-7.

32. Grimwade, L.F., Happerfield, L., Tristram, C., Mclntosh, G., Rees, M., Bench, A.J., Boyd, E.M., Hall, M., Quinn, A., Piggott, N., Scorer, P., Scott, M.A., Erber, W.N., Phospho-STAT5 and phospho-Akt expression in chronic myeloproliferative neoplasms. Br J Haematol, 2009. 147(4): p. 495-506.

33. Shide, K., Shimoda, H.K., Kumano, T., Karube, K., Kameda, T., Takenaka, K., Oku, S., Abe, H., Katayose, K.S., Kubuki, Y., Kusumoto, K., Hasuike, S., Tahara, Y., Nagata, K., Matsuda, T., Ohshima, K., Harada, M., Shimoda, K., Development of ET, primary myelofibrosis and PV in mice expressing JAK2 V617F. Leukemia, 2008. 22(1): p. 87-95.

34. Bakin, A.V., Tomlinson, A.K., Bhowmick, N.A., Moses, H.L., Arteaga, C.L., Phosphatidylinositol 3-kinase function is required for transforming growth factor beta-mediated epithelial to mesenchymal transition and cell migration. J Biol Chem, 2000. 275(47): p. 36803-10.

35. Nyga, R., Pecquet, C., Harir, N., Gu, H., Dhennin-Duthille, I., Régnier, A., Gouilleux-Gruart, V., Lassoued, K., Gouilleux, F., Activated STAT5 proteins induce activation of the PI 3-kinase/Akt and Ras/MAPK pathways via the Gab2 scaffolding adapter. Biochem J, 2005. 390(Pt 1): p. 359-66.

36. Mayerhofer, M., Valent, P., Sperr, W.R., Griffin, J.D., Sillaber, C., BCR/ABL induces expression of vascular endothelial growth factor and its transcriptional activator, hypoxia inducible factor-1alpha, through a pathway involving phosphoinositide 3-kinase and the mammalian target of rapamycin. Blood, 2002. 100(10): p. 3767-75.

37. Gianelli, U., Vener, C., Raviele, P.R., Savi, F., Somalvico, F., Calori, R., lurlo, A., Radaelli, F., Fermo, E., Bucciarelli, P., Bori, S., Coggi, G., Deliliers, G.L., VEGF expression correlates with microvessel density in Philadelphia chromosome-negative chronic myeloproliferative disorders. Am J Clin Pathol, 2007. 128(6): p. 966-73.

38. Panteli, K., Zagorianakou, N., Bai, M., Katsaraki, A., Agnantis, N.J., Bourantas, K., Angiogenesis in chronic myeloproliferative diseases detected by CD34 expression. Eur J Haematol, 2004. 72(6): p. 410-5.

39. Boveri, E., Passamonti, F., Rumi, E., Pietra, D., Elena, C., Arcaini, L., Pascutto, C., Castello, A., Cazzola, M., Magrini, U., Lazzarino, M., Bone marrow microvessel density in chronic myeloproliferative disorders: a study of 115 patients with clinicopathological and molecular correlations. Br J Haematol, 2008. 140(2): p. 162-8.

40. Arora, B., Ho, C.L., Hoyer, J.D., Mesa, R.A., Tefferi, A., Bone marrow angiogenesis and its clinical correlates in myelofibrosis with myeloid metaplasia. Haematologica, 2004. 89(12): p. 1454-8.

41. Steurer, M., Zoller, H., Augustin, F., Fong, D., Heiss, S., Strasser-Weippl, K., Gastl, G., Tzankov, A., Increased angiogenesis in chronic idiopathic myelofibrosis: vascular endothelial growth factor as a prominent angiogenic factor. Hum Pathol, 2007. 38(7): p. 1057-64.

42. Duhrsen, U. and Hossfeld, D.K., Stromal abnormalities in neoplastic bone marrow diseases. Ann Hematol, 1996. 73(2): p. 53-70.

43. Scadden, D.T., The stem cell niche in health and leukemic disease. Best Pract Res Clin Haematol, 2007. 20(1): p. 19-27. 



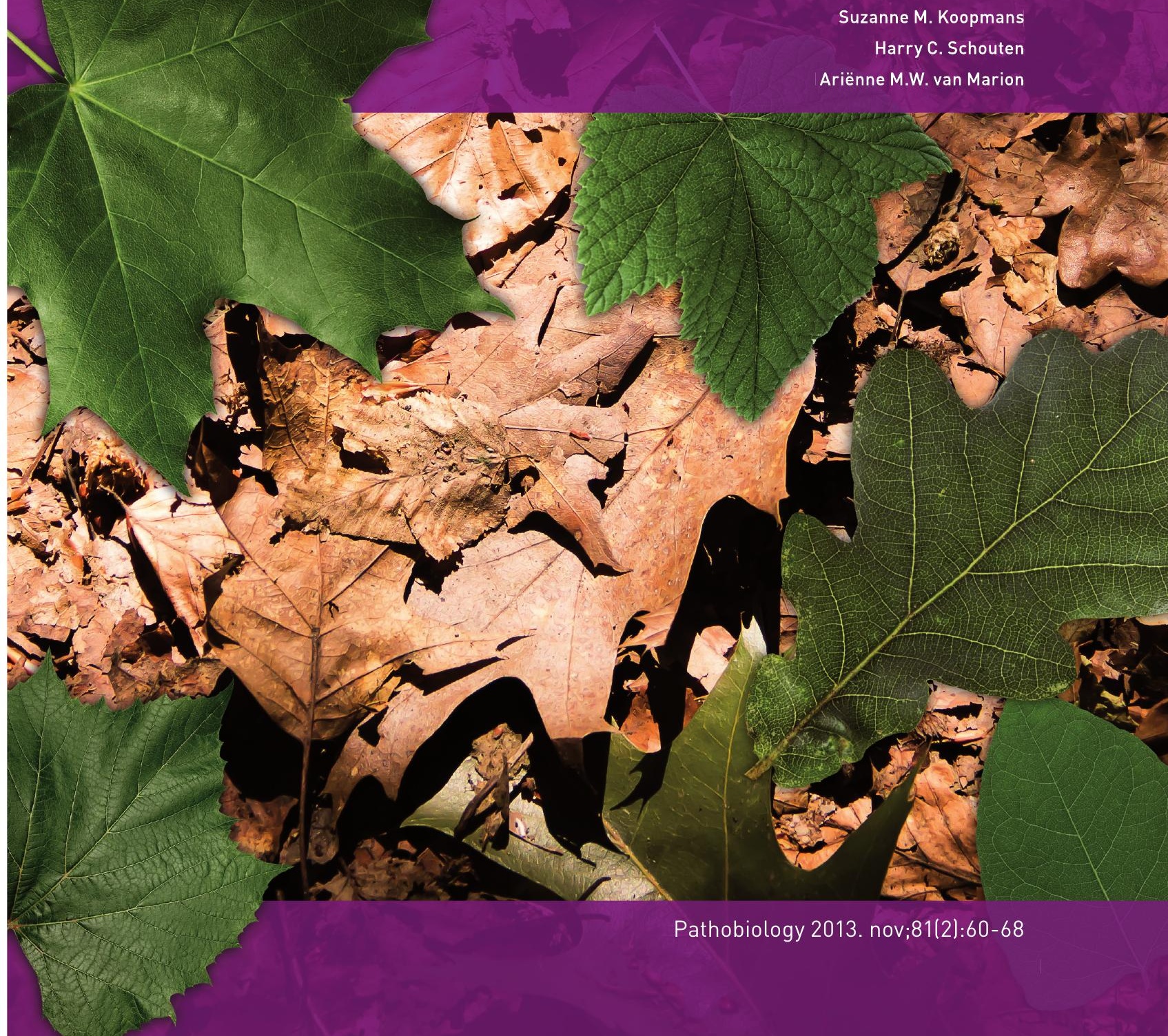




\section{ABSTRACT}

Background: Proliferative activity contributes to bone marrow cellularity in myeloproliferative neoplasia (MPN). Megakaryocytes are the most important cells in MPN bone marrow pathology. JAK2 $2^{\mathrm{V} 17 F}$ mutation constitutively activates JAK2, phosphorylating extracellular signal-regulated kinase (pErk) and phosphatidylinositol 3-kinase (PI3K)-Akt signalling pathway. Erk is involved in megakaryocyte differentiation, PI3K-Akt inhibits megakaryocyte apoptosis via Bcl-xL and two downstream effectors p70S6k and Bnip3. Immunohistochemic expression of phosphorylated Erk, Akt, p70S6k and Bnip3 was studied along with microvessel density (MVD) in MPN bone marrow and megakaryocytes.

Methods: 36 essential thrombocythemia (ET), 25 polycythemia vera (PV) and 45 primary myelofibrosis (PMF) patients, were analyzed for pErk, pAkt, Bnip3, p70S6k and MVD expression by immunostaining bone marrow biopsy sections followed by automated image analysis. JAK2 ${ }^{\mathrm{V} 617 F}$ was analysed through real time PCR in blood samples.

Results: pErk and pAkt were significantly higher expressed in MPN megakaryocytes, mainly ET patients, compared to controls. Bnip3 was higher expressed in bone marrow of control patients and in MPN megakaryocytes. MPN megakaryocytes, mainly ET patients, showed higher p70S6k expression compared to controls.

Conclusion: Increased bone marrow cellularity in MPN patients might be influenced by increased pErk, pAkt and decreased Bnip3 expression. A dominant role for megakaryocytes in ET patients was shown. Increased amounts of megakaryocytes in MPN patients can be due to increased pAkt and p70S6k. 


\section{INTRODUCTION}

Myeloproliferative neoplasia (MPN) are clonal bone marrow stem cell disorders originating from a multipotent haematopoietic stem cell characterized by proliferation of the myeloid, erythroid and/or megakaryocytic cell lineages resulting in increased numbers of granulocytes, erythrocytes or platelets in the peripheral blood. MPNs can be divided in chronic myelogenous leukaemia (CML) carrying the Philadelphia $(\mathrm{Ph}+)$ chromosome as a result of $\mathrm{t}(9 ; 22)$ and diseases which do not carry the Philadelphia chromosome (Ph-). ${ }^{[1]}$ The three most commonly occurring classical Ph negative MPN (Ph- MPN) are essential thrombocythemia (ET), polycythemia vera (PV) and primary myelofibrosis (PMF). ${ }^{[2,3]}$

In 2005 a mutation in the JAK2 gene was detected. The JAK2 ${ }^{\text {V617F }}$ mutation, which substitutes valine for phenylalanine and disrupts the inhibitory function of the pseudokinase domain in JAK2, constitutively activating the JAK2 gene..$^{[4-7]}$ The activating JAK2 ${ }^{\mathrm{V} 177}$ mutation is involved in several different pathways; in the mitogen-activated protein kinase (MAPK) signalling pathway, the phosphatidylinositol 3-kinase (PI3K)-Akt signalling pathway and the activation of the STAT (signal transducer and activator of transcription) family. ${ }^{[5,8]}$ Besides pathways influenced by the mutated JAK2 gene both pro- and anti-apoptotic proteins in general could be involved in the pathogenesis of Ph- MPN.

First, JAK2 can activate the receptor tyrosine kinase-Grb2-SOS signalling axis, activating Ras GTPase and Raf, continuing in activating MEK and the extracellular signal-regulated kinase (Erk) signalling pathway. The Erk signalling pathway is not only activated by JAK2 but can also be activated by numerous extracellular signals. ${ }^{[8-10]}$ Several studies have shown the involvement of the Erk pathway in megakaryocyte differentiation. ${ }^{[11-13]}$ Therefore, we examined the phosphorylation status of Erk (pErk) in bone marrow trephines of Ph- MPN patients and in megakaryocytes.

Second, the phosphatidylinositol 3-kinase (PI3K)-Akt signalling pathway is activated by the JAK2 mutation. Akt is an important mediator in the PI3K pathway and is involved in many cellular processes including inhibiting apoptosis, protein synthesis and cell differentiation and metabolism. It is already known that Akt is constitutively activated in acute myeloid leukaemia (AML). ${ }^{[14-18]}$ The PI3K-Akt pathway is activated by growth factors and cytokines resulting in phosphorylation of Akt, which up-regulates $\mathrm{Bcl-xL}$ leading to inhibition of megakaryocyte apoptosis. ${ }^{[19]}$ The mammalian target of rapamycine (mTor) is a serine/threonine kinase which is an effector protein of Akt. ${ }^{[20]} \mathrm{Akt}$ and $\mathrm{mTor}$ are both necessary for the activation of ribosomal p70S6 kinase (p70S6k). ${ }^{[21]}$ Therefore, we also examined the phosphorylation status of Akt (pAkt) and p70S6k in bone marrow trephines of Ph- MPN patient.

Third, besides the Akt signalling pathway, also anti-apoptotic proteins could be important factors in the development of Ph- MPN. The Bcl-2 protein family consist of both pro- and anti- 
apoptotic proteins, depending on the different combinations of the $\mathrm{Bcl}-2$ homology domains. Bnip3 is a pro-apoptotic protein belonging to the Bcl-2 family. Bnip3 is activated under hypoxic conditions in normal and cancer tissue upon hypoxia-inducible factor-1 $\alpha^{[22-24]}$

In the present study, we investigated the Erk and PI3K-Akt pathway and the expression of Bnip3 and p70S6k along with microvessel density (MVD) by immunohistochemistry on trephine biopsies to characterize abnormal activation of these pathways and abnormal apoptotic responses in bone marrow and megakaryocytes of Ph- MPN patients.

\section{MATERIALS AND METHODS}

\section{Study population}

The study was carried out on bone marrow trephines obtained from patients recorded at the Maastricht University Medical Centre, Maastricht, between January 1992 and December 2009, recorded at the Haga Hospital, The Hague, between January 2006 and December 2009 and recorded at the VieCuri Medical Centre, Venlo, between January 2005 and July 2010. The study was approved by the local institutional ethics committee. The study population consisted of 106 patients with a myeloproliferative neoplasm, with a mean age of 63.6 years (SD \pm 14.7 ) ranging from 17 to 86 years at time of diagnosis. None of the patients received therapy when the biopsy was taken. The patient population included in the study consisted of 36 ET (33.9\%), 25 PV (23.6\%), and 45 PMF (42.5\%) patients. All patients were clinically and histological diagnosed according to the World Health Organization (WHO) classification 2008. ${ }^{[25]}$ As shown in Table 1 and 2 a total of 61 patients (57.5\%) were women and 45 (42.5\%) were men. Fifty-six patients were carriers of the JAK2 $2^{\mathrm{V} 617 F}$ mutation (19 ET, $17 \mathrm{PV}$ and 20 PMF patients), 24 patients were carriers of the JAK2 wild type gene (15 ET, two PV and seven PMF patients) and of 26 patients the JAK2 ${ }^{\text {V617F }}$ mutational status was unknown due to insufficient DNA to detect the JAK2 status by PCR and because patients died prior to the availability of the JAK2 $2^{\text {V617F }}$ mutation test.

The patients were subdivided for the grading of myelofibrosis ( $\mathrm{mf}$ ) into $\mathrm{mf} 0 / 1$ and $\mathrm{mf} 2 / 3 ; 43$ patients belonged to the $\mathrm{mf} 0 / 1$ group (19 ET, $12 \mathrm{PV}, 12 \mathrm{PMF}$ ) of which 24 JAK2 ${ }^{\mathrm{V} 617 F}$ positive and 11 JAK2 wild type and 61 belonged to the $\mathrm{mf} 2 / 3$ group (17 ET, $12 \mathrm{PV}, 32 \mathrm{PMF}$ ) of which 31 JAK2 $2^{\mathrm{V} 17 \mathrm{~F}}$ positive and 13 JAK2 wild type.

The control group consisted of 36 morphologically normal negative staging biopsies from patients with non-Hodgkin lymphoma and Hodgkin lymphoma with a mean age of 55.8 years. 
THE ANTI-APOPTOTIC PATHWAYS IN BONE MARROW AND MEGAKARYOCYTES IN MYELOPROLIFERATIVE NEOPLASIA I

Table 1: Clinical and laboratory findings of patients with ET, PV and PMF and the control group.

\begin{tabular}{|c|c|c|c|c|}
\hline & $\begin{array}{c}\text { Essential } \\
\text { thrombocythemia } \\
n=36\end{array}$ & $\begin{array}{l}\text { Polycythemia } \\
\text { vera } \\
n=25\end{array}$ & $\begin{array}{c}\text { Primary } \\
\text { myelofibrosis } \\
n=45\end{array}$ & $\begin{array}{c}\text { Control bone } \\
\text { marrow } \\
n=36\end{array}$ \\
\hline Males / females & $11 / 25$ & $8 / 17$ & $26 / 19$ & $23 / 13$ \\
\hline Age, y, mean (SD) & $59(17,70)$ & $65(13,56)$ & $67(10,73)$ & $56(14,33)$ \\
\hline $\begin{array}{l}\text { JAK2 wild type / } \\
\text { JAK2 mutation / } \\
\text { JAK2 unknown }\end{array}$ & $\begin{array}{c}15 \\
19 \\
2\end{array}$ & $\begin{array}{c}2 \\
17 \\
6\end{array}$ & $\begin{array}{c}7 \\
20 \\
18\end{array}$ & $\begin{array}{c}36 \\
0 \\
0\end{array}$ \\
\hline $\begin{array}{l}\text { White blood cell } \\
\text { count* } 10^{9} / L \text {, mean (SD) }\end{array}$ & $9,37(2,89)$ & $16,08(12,22)$ & $11,89(11,38)$ & $8,16(4,44)$ \\
\hline Minimum-maximum & $4,4-18,30$ & $5,70-62,00$ & $0,90-70,60$ & $2,80-23,80$ \\
\hline $\begin{array}{l}\text { Haemoglobin, } \\
\mathrm{mmol} / \mathrm{L} \text {, mean (SD) }\end{array}$ & $8,53(1,26)$ & $9,89(1,81)$ & $7,09(1,58)$ & $8,16(1,10)$ \\
\hline Minimum-maximum & $6,10-12,00$ & $6,70-13,50$ & $3,30-10,60$ & $6,10-11,10$ \\
\hline $\begin{array}{l}\text { Haematocrit L/L, } \\
\text { mean (SD) }\end{array}$ & $0,43(0,06)$ & $0,52(0,09)$ & $0,35(0,08)$ & $0,39(0,05)$ \\
\hline Minimum-maximum & $0,29-0,61$ & $0,37-0,68$ & $0,15-0,50$ & $0,30-0,52$ \\
\hline $\begin{array}{l}\text { Thrombocytes*10\%, } \\
\text { mean (SD) }\end{array}$ & 929 [346] & 662 (316) & 564 (532) & 263 (137) \\
\hline Minimum-maximum & $327-1862$ & $112-1371$ & $15-2644$ & 49-585 \\
\hline
\end{tabular}

Table 2: Clinical and laboratory findings of patients with the JAK2 mutation or with wild type JAK2.

\begin{tabular}{|c|c|c|}
\hline & JAK2 positive $n=56$ & Wild type JAK2 n=24 \\
\hline Males / females & $26 / 30$ & $9 / 15$ \\
\hline $\begin{array}{l}\text { Age, } y, \\
\text { mean (SD) }\end{array}$ & $64(14,96)$ & $63(14,28)$ \\
\hline $\begin{array}{l}\text { White blood cell } \\
\text { count* } 10^{9} / L \text {, mean (SD) }\end{array}$ & $13,6(9,64)$ & $8,5(3,76)$ \\
\hline $\begin{array}{l}\text { Haemoglobine, } \mathrm{mmol} / \mathrm{L} \text {, } \\
\text { mean (SD) }\end{array}$ & $8,91(1,46)$ & $7,81(1,67)$ \\
\hline $\begin{array}{l}\text { Haematocrit L/L, } \\
\text { mean (SD) }\end{array}$ & $0,44(0,08)$ & $0,39(0,09)$ \\
\hline $\begin{array}{l}\text { Thrombocytes*10\%/L, } \\
\text { mean (SD) }\end{array}$ & 728 (341) & 907 (374) \\
\hline
\end{tabular}




\section{Immunohistochemistry}

The bone marrow biopsy specimens were decalcificated using the Kristensen procedure for one hour or EDTA decalcification for four hours, followed by standard tissue processing and paraffin embedding. From the paraffin-embedded blocks $3 \mu \mathrm{m}$ slices were cut and mounted on starfrost slides (Knittel Gläser, Germany). All the antibodies were tested for specificity on positive and negative tumour control slides and also individually tested on decalcified control bone marrow biopsies, resulting in a variation of immunohistochemical procedures, optimised for all individual antibodies.

Immunohistochemical staining of pErk, pAkt, Bnip3 and p70S6K was carried out using the antihuman rabbit monoclonal antibody phospho-p44/42MAPK (Thr202/Tyr204), phosphoAkt (Ser473), antihuman mouse monoclonal antibody anti-Bnip3 (B7931) and phospho-70 S6 kinase (Thr389) (1A5) with a dilution of 1:100, 1:25, 1:300 and 1:100 respectively (Cell signalling Technology, Danvers, for pErk, pAkt and p70S6k, Sigma-Aldrich, USA, for Bnip3). After deparaffinization the slides were put in $0.3 \% \mathrm{H}_{2} \mathrm{O}_{2}$ (Bnip3, p70S6k) in methanol to block endogenous peroxidase activity, followed by antigen retrieval by boiling for 20 minutes in $10 \mathrm{mM}$ sodium citrate buffer $\mathrm{pH} 6.0$ in a water bath of $100^{\circ} \mathrm{C}$. For pErk and pAkt after deparaffinization and antigen retrieval by boiling for 20 minutes in $10 \mathrm{mM}$ sodium citrate buffer $\mathrm{pH} 6.0$ in a water bath of $100^{\circ} \mathrm{C}$, endogenous peroxidase activity was blocked in $3 \%$ $\mathrm{H}_{2} \mathrm{O}_{2}$ in methanol. After blocking solution (3\% Bovine Serum Albumin/Phosphate Buffered Saline (BSA/PBS) for Bnip3 and p70S6k, Tris Buffered Saline Tween (TBST)/5\% goat serum $\mathrm{pH}$ 7.2-7.6 for pErk and pAkt) the primary antibody, applied in TBST/1\% BSA (pH7.2-7.6 for pErk, pAkt) or antibody diluent (Dako, Denmark, for Bnip3, p70S6k) was incubated overnight at $4^{\circ} \mathrm{C}$ (pErk, pAkt) or for 1 hour at room temperature (Bnip3, p70S6k). The slides were then incubated for 30 minutes with Powervision poly-HRP-Anti Ms/Rb/Ra IgG a histostaining kit (ImmunoLogic, The Netherlands, pErk, pAkt) or with Envision (Dako Real ${ }^{\mathrm{TM}}$ Envision $^{\mathrm{TM}}$ Detection system (K5007), Bnip3, p70S6k). After developing the colour, with freshly made diaminobenzidine solution (Dako), slides were counterstained with haematoxylin (Merck, Whitehouse Station, NJ), dehydrated and mounted in Entellan (Merck).

The 142 trephines (MPN patients plus control patients) were immunohistochemically analysed using an automated immunostainer (Dako autostainer Link 48) with CD34 (clone QBend 10, Dako). CD34 was incubated for 20 minutes at room temperature. The reaction was revealed by means of the Dako Envision Flex Kit (Dako) according to the manufacturer's instructions.

\section{Quantification of staining}

The amount of positive stained cells for pErk, pAkt, Bnip3 and p70S6k (see Figure 1) was quantified using an image processing and analysis system (Leica, Cambridge, UK) linked to 
a Leica DML3000 light microscope (Leica Quantimet, Germany). The program used in this system was QWin (Leica's Windows-based image analysis tool-kit-Leica, Cambridge, UK). was used for postprocessing. For measuring pErk, pAkt, Bnip3 and p70S6k, five hot spots per slide were included to measure total tissue, total nuclei positive for pErk, pAkt, Bnip3 or p70S6k and total nuclei count. The amount of positivity was calculated as the percentage of positive nuclear pixels related to the total number of nuclear pixels.

Figure 1: Examples of pErk, pAkt, Bnip3 and p70S6k staining in MPN patients and control patients.

A. pErk (HE, 1000x) staining in MPN patient B. pErk (HE, 650x) staining in control patient C. pAkt (HE, 630x) staining in MPN patient D. pAkt $(\mathrm{HE}, 650 \mathrm{x})$ staining in control patient $\mathrm{E}$. Bnip3 (HE, 1000x) in MPN patient F. Bnip3 (HE, 650x) in control patient G. p70S6k (HE, 1000x) in MPN patient H. p70S6k (HE, 650x) in control patient.
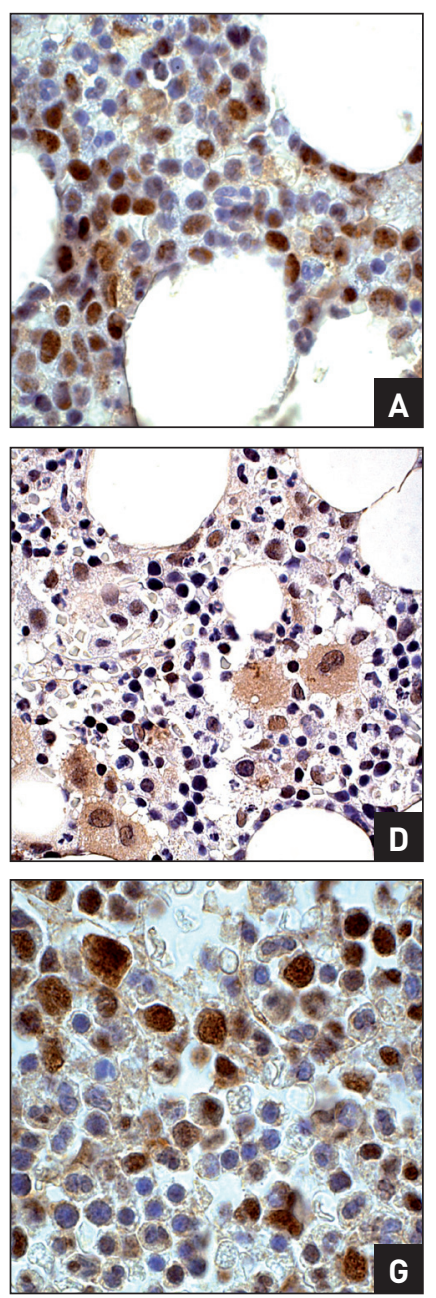
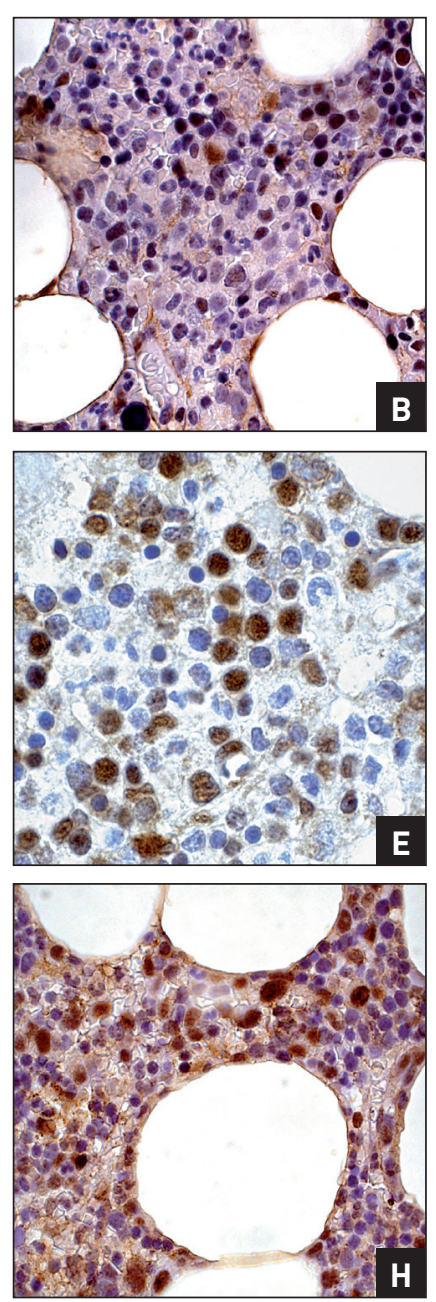
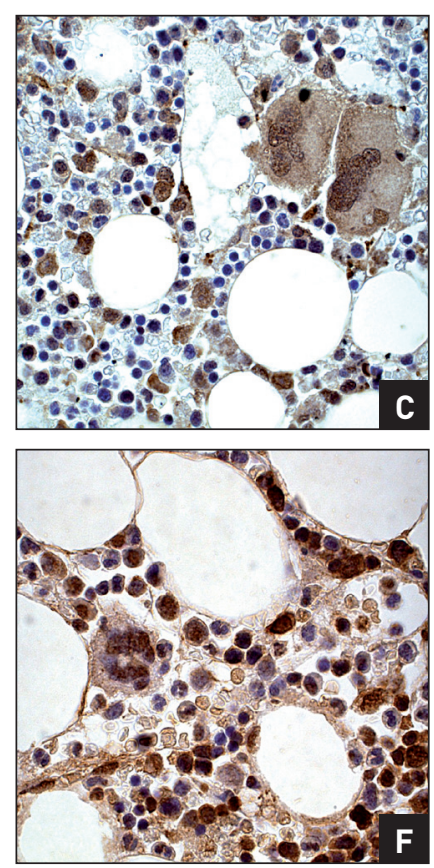
From the five pictures taken we counted the total amount of megakaryocytes, the total amount of positive stained megakaryocytes, the megakaryocytes with nuclear staining and with cytoplasmatic staining. The mean total amount of megakaryocytes, total amount of positive stained megakaryocytes, megakaryocytes with nuclear staining and with cytoplasmatic staining was calculated using Excel (Microsoft, Redmond, WA).

MVD was assessed by counting the number of CD34 positive capillary-, arteriolar- or sinuslumen in five $1 \mathrm{~mm}^{2}$ fields at 100x magnification, calculating the mean over these five fields. The grading of fibrosis was done according to the European consensus on grading of bone marrow fibrosis. ${ }^{[26]}$

\section{Statistical analysis}

The data were statistically evaluated using the SPSS 15 statistical package, analyzed descriptively (descriptives, explore and crosstabs). Statistical comparison was performed by Mann-Whitney $U$-test when comparing medians and by the independent t-test to evaluate differences in clinical parameters between the different groups. Differences were considered significant when $p$-value was less then 0.05. Pearson's test was performed for correlating the expression of pErk with MVD, pAkt with MVD, p70S6k with MVD, Bnip3 with MVD and fibrosis grading with MVD.

In some cases bone marrow tissue was lost during the pre-treatment of the slides; for pErk we report 4 missing values, for pAkt 5, for p70S6k 20, for Bnip3 21 and for MVD 5 missing values. For the grading of myelofibrosis we report 2 missing values.

\section{RESULTS}

The results of all staining percentages are summarized in Table 3, 4, 5, 6 and 7. Qualitative microscopic evaluation of pErk showed a nuclear expression predominantly in the erythroblasts and occasionally in endothelial cells and plasma cells. The granulopoiesis and myelopoiesis did not show any pErk expression. Compared to the control group pErk was significantly higher expressed in the general bone marrow of ET patient ( $p=0.013$ ) and the total MPN group ( $p=0.028$ ) The megakaryocytes expressed $p$ Erk significantly higher in ET ( $p=0.000), P V(p=0.000)$ and PMF patients $(p=0.000)$ and the total MPN group ( $p=0.000$ ) compared to the megakaryocytes of the control group. As shown in Table 2, there were significantly more stained megakaryocytes in the ET and PV group compared to the control group.

pAkt was expressed in the cytoplasm and nucleus of immature myeloid cells. The megakaryocytes expressed pAkt cytoplasmatic. pAkt was significantly higher expressed in megakaryocytes of ET $(p=0.000), P V(p=0.000)$ and PMF patients $(p=0.000)$ and the total MPN group $(p=0.000)$ compared 
to the megakaryocytes of the control group. The megakaryocytes of ET patients showed a significant higher pAkt expression compared to PV ( $p=0.035)$ and PMF ( $p=0.050)$ patients. Bnip3 showed its nuclear and cytoplasmatic expression in the myeloid cells. The megakaryocytes expressed Bnip3 in the cytoplasm and the endothelial cells nuclear. The expression of Bnip3 in the general bone marrow was comparably higher in the control group, this was statistically significant between ET patients and the controls ( $p=0.003$ ), between PMF patients and the controls ( $p=0.001)$ and between the total MPN group and the control group ( $p=0.001)$. However, the megakaryocytes expressed Bnip3 significantly higher in ET ( $p=0.000), \mathrm{PV}(p=0.000)$ and PMF patients $(p=0.000)$ and the total MPN group $(p=0.000$ ) compared to the megakaryocytes of the control group. Bnip3 expression was significantly higher in megakaryocytes of ET patients compared to megakarocytes of PV ( $p=0.019)$.

The expression of p70S6k was mainly in the immature myeloid (nuclear) and mature myeloid cell line (nuclear and cytoplasmatic). Megakaryocytes expressed p70S6k in the cytoplasm and in the nucleus. Adipocytes expressed p70S6k as well. p70S6k was significantly higher expressed in megakaryocytes of ET $(p=0.000), \mathrm{PV}(p=0.000)$ and PMF patients $(p=0.000)$ and the total MPN group ( $p=0.000$ ) compared to megakaryocytes of the control group. The megakaryocytes of ET patients showed a significant higher p70S6k expression compared to megakaryocytes of PV ( $p=0.003$ ) and PMF patients ( $p=0.008$ ).

Concerning the myelofibrosis grading and the stainings we report a statistically significant higher p70S6k expression in the $\mathrm{mf} 0 / 1$ group ( $p=0.033$ ) compared to the $\mathrm{mf} 2 / 3$ group. For MVD there was higher expression of MVD in the $\mathrm{mf} 2 / 3$ group ( $p=0.001$ ) compared to the $\mathrm{mf} 0 / 1$ group. The pearson correlation also showed a significant correlation of MVD with the grading of myelofibrosis $(p=0.000)$.

\section{DISCUSSION}

In this study we examined the immunohistochemical expression of pErk, pAkt, Bnip3 and p70S6k in total bone marrow cells and in megakaryocytes of ET, PV, PMF and control patients along with the MVD.

Activated Erk activates BAD, an apoptosis activator, and Bcl-2, an apoptosis inhibitor. ${ }^{\text {[27, 28] }}$ The net result of Erk phosphorylation is an overall inhibition of apoptosis. In our study we saw higher $\mathrm{pErk}$ expression in bone marrow of MPN patients, suggesting this can be responsible for the increased bone marrow cellularity seen in MPN patients. Although it was shown that Erk is constitutively activated by the JAK2 ${ }^{\mathrm{V} 617 \mathrm{~F}}$ mutation, we failed to show a significant pErk increase in JAK2 ${ }^{\mathrm{V} 617 F}$ positive patients. This might be due to the relative high number of patients with an unknown JAK2 status in our study. The overall higher pErk expression in 
Table 3: Percentage of stained megakaryocytes in ET, PV and PMF patients versus the control group.

\begin{tabular}{|c|c|c|c|c|}
\hline & $\begin{array}{c}\text { Essential } \\
\text { thrombocythemia } \\
n=36\end{array}$ & $\begin{array}{l}\text { Polycythemia } \\
\text { vera } \\
n=25\end{array}$ & $\begin{array}{c}\text { Primary } \\
\text { myelofibrosis } \\
n=45\end{array}$ & $\begin{array}{c}\text { Control bone } \\
\text { marrow } \\
n=36\end{array}$ \\
\hline $\begin{array}{l}\text { pErk, positive stained megakaryocytes, } \\
\% \text {, mean (SD), } \\
\text { t-test } p \text {-value }\end{array}$ & $\begin{array}{l}80,13 \\
(26,67) \\
p=0,02\end{array}$ & $\begin{array}{l}84,25 \\
(25,67) \\
p=0,01\end{array}$ & $\begin{array}{l}75,61 \\
(30,92) \\
p=0,10\end{array}$ & $\begin{array}{c}63,62 \\
(32,69)\end{array}$ \\
\hline $\begin{array}{l}\text { pAkt, positive stained megakaryocytes, } \\
\% \text {, mean (SD), } \\
\text { t-test } p \text {-value }\end{array}$ & $\begin{array}{l}93,19 \\
(8,63) \\
p=0,06\end{array}$ & $\begin{array}{l}83,97 \\
(24,75) \\
p=0,85\end{array}$ & $\begin{array}{l}84,81 \\
(21,44) \\
p=0,72\end{array}$ & $\begin{array}{c}82,58 \\
(30,38)\end{array}$ \\
\hline $\begin{array}{l}\text { pBnip3, positive stained megakaryocytes, } \\
\% \text {, mean (SD), } \\
\text { t-test } p \text {-value }\end{array}$ & $\begin{array}{l}97,04 \\
(6,27) \\
p=0,07\end{array}$ & $\begin{array}{c}87,72 \\
(23,23) \\
p=0,80\end{array}$ & $\begin{array}{l}83,00 \\
(29,67) \\
p=0,66\end{array}$ & $\begin{array}{c}86,29 \\
(30,92)\end{array}$ \\
\hline $\begin{array}{l}\text { p70S6k, positive stained megakaryocytes, } \\
\% \text {, mean (SD) } \\
\text { t-test } p \text {-value }\end{array}$ & $\begin{array}{l}95,92 \\
(7,38) \\
p=0,12\end{array}$ & $\begin{array}{l}86,79 \\
(22,70) \\
p=0,77\end{array}$ & $\begin{array}{l}87,77 \\
(19,65) \\
p=0,87\end{array}$ & $\begin{array}{c}88,63 \\
(24,58)\end{array}$ \\
\hline $\begin{array}{l}\text { VEGF, positive stained megakaryocytes, } \\
\% \text {, mean (SD), } \\
\text { t-test } p \text {-value }\end{array}$ & $\begin{array}{l}84,59 \\
(20,97) \\
p=0,57\end{array}$ & $\begin{array}{l}79,32 \\
(26,00) \\
p=0,80\end{array}$ & $\begin{array}{c}86,80 \\
(22,67) \\
p=0,35\end{array}$ & $\begin{array}{c}81,13 \\
(27,67)\end{array}$ \\
\hline
\end{tabular}

Table 4: Percentage pErk, pAkt, Bnip3, p70S6K and MVD in ET, PV, PMF, all MPN patients and control patients in total bone marrow cells.

\begin{tabular}{|c|c|c|c|c|c|c|c|c|c|c|}
\hline \multirow[b]{2}{*}{$\begin{array}{l}\text { pErk, \%, }{ }^{*} \text { mean (SD) } \\
\text { Minimum-maximum } \\
\text { (CI) }\end{array}$} & \multicolumn{2}{|c|}{$\begin{array}{c}\text { Essential } \\
\text { thrombocythemia } \\
n=36\end{array}$} & \multicolumn{2}{|c|}{$\begin{array}{l}\text { Polycythemia } \\
\text { vera } \\
n=25\end{array}$} & \multicolumn{2}{|c|}{$\begin{array}{c}\text { Primary } \\
\text { myelofibrosis } \\
n=45\end{array}$} & \multicolumn{2}{|c|}{$\begin{array}{c}\text { All MPN } \\
\text { patients } \\
n=106\end{array}$} & \multicolumn{2}{|c|}{$\begin{array}{c}\text { Control } \\
\text { patients } \\
n=36\end{array}$} \\
\hline & $\begin{array}{r}16,93 \\
1,88 \\
112,37\end{array}$ & $\begin{aligned} & (11,04) \\
- & 36,68 \\
- & 21,34]\end{aligned}$ & $\begin{array}{r}13,49 \\
2,10 \\
(10,14\end{array}$ & $\begin{aligned} & (8,73) \\
- & 31,44 \\
- & 19,71)\end{aligned}$ & $\begin{array}{r}14,61 \\
3,24 \\
112,52\end{array}$ & $\begin{aligned} & (7,66) \\
- & 30,23 \\
- & 19,16)\end{aligned}$ & $\begin{array}{r}15,13 \\
1,88 \\
(13,74\end{array}$ & $\begin{aligned} & (9,21) \\
- & 36,68 \\
- & 18,36)\end{aligned}$ & $\begin{array}{r}11,41 \\
4,09 \\
111,74\end{array}$ & $\begin{aligned} & (6,70) \\
- & 34,32 \\
- & 20,34]\end{aligned}$ \\
\hline $\begin{array}{l}\text { pAkt, \%, }{ }^{*} \text { mean (SD) } \\
\text { Minimum-maximum } \\
\text { (CI) }\end{array}$ & $\begin{array}{r}15,31 \\
4,93 \\
112,44\end{array}$ & $\begin{aligned} & (6,17) \\
- & 29,85 \\
- & 17,33)\end{aligned}$ & $\begin{array}{r}14,04 \\
0,99 \\
(13,47\end{array}$ & $\begin{aligned} & (7,51) \\
- & 29,18 \\
- & 20,90)\end{aligned}$ & $\begin{array}{r}13,99 \\
0,71 \\
(12,15\end{array}$ & $\begin{aligned} & (6,01) \\
- & 27,76 \\
- & 16,80)\end{aligned}$ & $\begin{array}{r}14,43 \\
2,79 \\
(13,74\end{array}$ & $\begin{aligned} & (6,42) \\
- & 29,18 \\
- & 16,70)\end{aligned}$ & $\begin{array}{r}10,11 \\
1,04 \\
111,25\end{array}$ & $\begin{aligned} & (7,19) \\
- & 27,17 \\
-\quad & 19,15)\end{aligned}$ \\
\hline $\begin{array}{l}\text { Bnip3, \%, }{ }^{*} \text { mean (SD) } \\
\text { Minimum-maximum } \\
\text { (CI) }\end{array}$ & $\begin{array}{r}15,55 \\
4,02 \\
(12,79\end{array}$ & $\begin{aligned} & (5,72) \\
- & 25,46 \\
- & 17,42)\end{aligned}$ & $\begin{array}{r}18,20 \\
6,46 \\
115,70\end{array}$ & $\begin{aligned} & (6,53) \\
- & 29,89 \\
- & 23,01)\end{aligned}$ & $\begin{array}{r}15,27 \\
6,09 \\
112,30\end{array}$ & $\begin{aligned} & (5,27) \\
- & 26,03 \\
- & 17,07)\end{aligned}$ & $\begin{array}{r}16,10 \\
4,02 \\
114,35\end{array}$ & $\begin{aligned} & (5,83) \\
- & 29,89 \\
- & 17,36)\end{aligned}$ & $\begin{array}{r}20,09 \\
11,18 \\
(13,48\end{array}$ & $\begin{aligned} & (5,28) \\
- & 28,91 \\
- & 20,57)\end{aligned}$ \\
\hline $\begin{array}{l}\text { p70S6K, \%,* mean (SD) } \\
\text { Minimum-maximum } \\
\text { (CI) }\end{array}$ & $\begin{array}{r}20,74 \\
10,87 \\
(18,67\end{array}$ & $\begin{aligned} & (5,25) \\
- & 33,63 \\
- & 22,44)\end{aligned}$ & $\begin{array}{r}22,54 \\
9,33 \\
\lfloor 17,31\end{array}$ & $\begin{aligned} & (7,28) \\
- & 33,27 \\
- & 25,91)\end{aligned}$ & $\begin{array}{r}21,06 \\
8,54 \\
118,56\end{array}$ & $\begin{aligned} & (5,84) \\
- & 33,23 \\
- & 23,40)\end{aligned}$ & $\begin{array}{r}21,28 \\
8,54 \\
(19,53\end{array}$ & $\begin{aligned} & (5,97) \\
- & 33,27 \\
- & 22,36)\end{aligned}$ & $\begin{array}{r}20,07 \\
8,71 \\
116,94\end{array}$ & $\begin{aligned} & (4,44) \\
- & 30,16 \\
- & 22,35)\end{aligned}$ \\
\hline $\begin{array}{l}\text { MVD, } 1 \mathrm{~mm}^{2} \text {, mean (SD) } \\
\text { Minimum-maximum } \\
\text { (CI) }\end{array}$ & $\begin{array}{r}37,72 \\
3,40 \\
129,70\end{array}$ & $\begin{aligned} & (22,18) \\
- & 89,60 \\
- & 49,07)\end{aligned}$ & $\begin{array}{r}47,55 \\
5,80 \\
135,77\end{array}$ & $\begin{array}{r}(27,45) \\
-\quad 111,20 \\
-\quad 70,80)\end{array}$ & $\begin{array}{r}58,47 \\
12,80 \\
150,30\end{array}$ & $\begin{array}{r}(31,56) \\
-\quad 122,40 \\
-\quad 76,391\end{array}$ & $\begin{array}{r}48,79 \\
3,40 \\
144,16\end{array}$ & $\begin{array}{r}(28,92) \\
-\quad 122,40 \\
-\quad 59,031\end{array}$ & $\begin{array}{r}27,95 \\
5,60 \\
(17,94\end{array}$ & $\begin{aligned} & (11,25) \\
- & 57,80 \\
- & 34,27)\end{aligned}$ \\
\hline
\end{tabular}

* calculated as percentage positive nuclei of total nuclei count 
Table 5: Percentage pErk, pAkt, Bnip3, p70S6k and MVD in JAK2 positive and wild type JAK2 patients in total bone marrow cells.

\begin{tabular}{|c|c|c|c|}
\hline & $\begin{array}{c}\text { JAK2 positive } \\
n=56\end{array}$ & \multicolumn{2}{|c|}{$\begin{array}{c}\text { Wild type JAK2 } \\
n=24\end{array}$} \\
\hline $\begin{array}{l}\text { pErk, \%, mean (SD)* } \\
\text { Minimum-maximum } \\
\text { (CI) }\end{array}$ & $\begin{aligned} 17,10 & (9,89) \\
3,65 & -36,68 \\
(13,58 & -19,41)\end{aligned}$ & $\begin{array}{r}13,71 \\
1,88 \\
110,82\end{array}$ & $\begin{array}{ll} & (7,52) \\
- & 25,79 \\
- & 17,53)\end{array}$ \\
\hline $\begin{array}{l}\text { pAkt, \%, mean (SD)* } \\
\text { Minimum-maximum } \\
\text { (CI) }\end{array}$ & $\begin{aligned} 14,82 & (6,40) \\
3,47 & -29,18 \\
(13,03 & -6,71)\end{aligned}$ & $\begin{array}{r}16,89 \\
8,35 \\
113,72\end{array}$ & $\begin{array}{l}(5,45) \\
-\quad 23,30 \\
-\quad 17,90)\end{array}$ \\
\hline $\begin{array}{l}\text { Bnip3, \%, mean (SD)* } \\
\text { Minimum-maximum } \\
\text { (CI) }\end{array}$ & $\begin{aligned} 16,89 & (4,43) \\
4,02 & -28,22 \\
(15,49 & -18,65)\end{aligned}$ & $\begin{array}{r}17,84 \\
7,21 \\
115,25\end{array}$ & $\begin{array}{r} \\
-\quad(5,25) \\
-\quad 26,03 \\
-\quad 20,44)\end{array}$ \\
\hline $\begin{array}{l}\text { p70S6k, \%, mean (SD)* } \\
\text { Minimum-maximum } \\
\text { (CI) }\end{array}$ & $\begin{aligned} 22,74 & (5,73) \\
8,54 & -33,63 \\
(21,21 & -24,46)\end{aligned}$ & $\begin{array}{r}21,47 \\
13,76 \\
119,46\end{array}$ & $\begin{aligned} & (4,17) \\
- & 27,47 \\
- & 23,48)\end{aligned}$ \\
\hline $\begin{array}{l}\text { MVD, } 1 \mathrm{~mm} 2 \text {, mean (SD) } \\
\text { Minimum-maximum } \\
\text { (CI) }\end{array}$ & $\begin{aligned} 52,77 & (30,58) \\
3,60 & -122,40 \\
(44,72 & -63,18)\end{aligned}$ & $\begin{array}{r}49,01 \\
17,20 \\
140,64\end{array}$ & $\begin{array}{r}(25,98) \\
-\quad 111,20 \\
-\quad 65,24)\end{array}$ \\
\hline
\end{tabular}

* calculated as percentage positive nuclei of total nuclei count

Table 6: Absolute amount of positive stained megakaryocytes of pErk, pAkt, Bnip3 and p70S6K in ET, PV, PMF, all MPN patients and control patients.

\begin{tabular}{|c|c|c|c|c|c|}
\hline & $\begin{array}{c}\text { Essential } \\
\text { thrombocythemia } \\
n=36\end{array}$ & $\begin{array}{l}\text { Polycythemia } \\
\text { vera } \\
n=25\end{array}$ & $\begin{array}{c}\text { Primary } \\
\text { myelofibrosis } \\
n=45\end{array}$ & $\begin{array}{l}\text { All MPN } \\
\text { patients } \\
n=106\end{array}$ & $\begin{array}{l}\text { Control } \\
\text { patients } \\
n=36\end{array}$ \\
\hline $\begin{array}{l}\text { pErk, absolute positive } \\
\text { megakaryocytes, mean (SD) } \\
\text { Minimum-maximum } \\
\text { (CI) }\end{array}$ & $\begin{array}{cc}2,79 & (1,91) \\
0,00 & -7,00 \\
(2,06 & -3,51)\end{array}$ & $\begin{array}{ll}2,24 & (1,32) \\
0,60 & -5,60 \\
(1,62 & -2,86)\end{array}$ & $\begin{array}{cc}1,88 & (1,55) \\
0,00 & -5,20 \\
(1,32 & -2,44)\end{array}$ & $\begin{array}{ll}2,29 & (1,67) \\
0,00 & -7,00 \\
(1,93 & -2,66)\end{array}$ & $\begin{array}{ll}0,71 & (0,69) \\
0,00 & -3,40 \\
(0,46 & -0,96)\end{array}$ \\
\hline $\begin{array}{l}\text { pAkt, absolute positive } \\
\text { megakaryocytes, mean (SD) } \\
\text { Minimum-maximum } \\
\text { (CI) }\end{array}$ & $\begin{array}{cc}3,04 & (1,47) \\
0,80 & -7,60 \\
(2,48 & -3,60)\end{array}$ & $\begin{array}{ll}2,25 & (1,71) \\
0,00 & -7,80 \\
(1,45 & -3,05)\end{array}$ & $\begin{array}{cc}2,38 & (1,42) \\
0,40 & -5,80 \\
(1,87 & -2,89)\end{array}$ & $\begin{array}{ll}2,59 & (1,53) \\
0,00 & -7,80 \\
(2,25 & -2,92)\end{array}$ & $\begin{array}{ll}1,11 & (0,95) \\
0,00 & -4,00 \\
(0,76 & -1,46)\end{array}$ \\
\hline $\begin{array}{l}\text { Bnip3, absolute positive } \\
\text { megakaryocytes, mean (SD) } \\
\text { Minimum-maximum } \\
\text { (CI) }\end{array}$ & $\begin{array}{cc}2,43 & (1,31) \\
0,20 & -5,40 \\
(1,94 & -2,93)\end{array}$ & $\begin{array}{ll}1,85 & (1,19) \\
0,20 & -4,00 \\
(1,29 & -2,40)\end{array}$ & $\begin{array}{cc}1,95 & (1,47) \\
0,00 & -5,20 \\
(1,42 & -2,48)\end{array}$ & $\begin{array}{cc}2,10 & 1,35) \\
0,00 & -5,40 \\
(1,80 & -2,40)\end{array}$ & $\begin{array}{ll}0,68 & (0,46) \\
0,00 & -1,60 \\
(0,51 & -0,85)\end{array}$ \\
\hline $\begin{array}{l}\text { p70S6K, absolute positive } \\
\text { megakaryocytes, mean (SD) } \\
\text { Minimum-maximum } \\
\text { (CI) }\end{array}$ & $\begin{array}{cc}2,85 & (1,24) \\
0,60 & -5,80 \\
(2,38 & -3,32)\end{array}$ & $\begin{array}{ll}1,80 & (1,23) \\
0,00 & -5,80 \\
(1,22 & -2,37)\end{array}$ & $\begin{array}{cc}1,96 & (1,27) \\
0,00 & -6,80 \\
(1,50 & -2,41)\end{array}$ & $\begin{array}{ll}2,24 & (1,32) \\
0,00 & -6,80 \\
(1,94 & -2,53)\end{array}$ & $\begin{array}{cc}0,97 & (0,65) \\
0,00 & -2,80 \\
(0,73 & -1,21)\end{array}$ \\
\hline
\end{tabular}


Table 7: Absolute amount of positive stained megakaryocytes of pErk, pAkt, Bnip3 and p70S6K in JAK2 positive or wild type JAK2 patients.

\begin{tabular}{|c|c|c|}
\hline & $\begin{array}{c}\text { JAK2 positive } \\
n=56\end{array}$ & 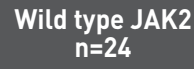 \\
\hline $\begin{array}{l}\text { pErk, absolute positive } \\
\text { megakaryocytes, mean (SD) } \\
\text { Minimum-maximum (CI) }\end{array}$ & $\begin{array}{cc}2,39 & (1,71) \\
0,00 & -6,60 \\
(1,88 & -2,90)\end{array}$ & $\begin{array}{rr}1,42 & (1,50) \\
0,00 & -7,00 \\
(1,00 & -1,85)\end{array}$ \\
\hline $\begin{array}{l}\text { pAkt, absolute positive } \\
\text { megakaryocytes, mean (SD) } \\
\text { Minimum-maximum (CI) }\end{array}$ & $\begin{array}{cc}2,67 & (1,61) \\
0,60 & -7,80 \\
(1,38 & -2,29)\end{array}$ & $\begin{array}{ll}1,84 & (1,61) \\
0,00 & -7,60 \\
(1,38 & -2,29)\end{array}$ \\
\hline $\begin{array}{l}\text { Bnip3, absolute positive } \\
\text { megakaryocytes, mean (SD) } \\
\text { Minimum-maximum (CI) }\end{array}$ & $\begin{array}{cc}2,16 & (1,37) \\
0,00 & -5,40 \\
(1,75 & -2,56)\end{array}$ & $\begin{array}{ll}1,31 & (1,13) \\
0,00 & -4,80 \\
(0,99 & -1,63)\end{array}$ \\
\hline $\begin{array}{l}\text { p70S6K, absolute positive } \\
\text { megakaryocytes, mean (SD) } \\
\text { Minimum-maximum (CI) }\end{array}$ & $\begin{array}{cc}2,30 & (1,46) \\
0,00 & -6,80 \\
(1,86 & -2,73)\end{array}$ & $\begin{array}{ll}1,52 & (1,06) \\
0,00 & -5,00 \\
(1,22 & -1,82)\end{array}$ \\
\hline
\end{tabular}

megakaryocytes of mainly ET but also PV and PMF patients and the total MPN group in our study might indicate a major important role for megakaryocytes in MPN pathogenesis and especially in ET patients. However, in ET patients, it might also be a result of the disease itself, while there was a significant higher amount of stained megakaryocytes in ET patients compared to the control group. Akt is phosphorylated by activated PI3K which in turn can be phosphorylated by PSTAT5 and the JAK2 V617F mutation (see Figure 2). The down stream effector of pAkt is the apoptosis inhibitor of megakaryocytes, Bcl-xL. ${ }^{[5,29-33]}$ Megakaryocytes of ET, PV and PMF patients and the total MPN group showed higher pAkt expression compared to the megakaryocytes of control patients in our study, with the highest expression in ET patients. This suggests a role for pAkt in the pathological increase in megakaryocytes seen in the bone marrow of MPN patients leading to an increase of platelets in the peripheral blood. Several studies ${ }^{[34,35]}$ showed an increased pAkt in JAK2 $2^{\text {V617F }}$ positive patients, however, we could not demonstrate that, probably due to the relative high number of patients with an unknown JAK2 status.

Bnip3 is a pro-apoptotic protein, which is activated under hypoxic conditions by Hif-1 (see Figure 3). ${ }^{[24]}$ The lower Bnip3 expression in the total group of MPN patients in our study might indicate that the increased bone marrow cellularity is also a result of decreased apoptosis and not only due to proliferative activity. A discrepancy seems to exist between our results of Bnip3 expression in total bone marrow cells and the Bnip3 expression in megakaryocytes of MPN patients. However, it might also refer to the protective role against bone marrow 
apoptosis; Bnip3 might contribute to the increased cellularity in total bone marrow cells.

For the activation of p70S6k the formation of a complex between the regulatory subunit of $\mathrm{PI3K}$ (p85) and $\mathrm{mTor}$ is require ${ }^{[21]}$ and is therefore in line with the higher pAkt expression in megakaryocytes of MPN patients in our study. Activated p70S6k phosphorylates BAD resulting in inactivation of BAD leading to inhibition of apoptosis (see Figure 3). ${ }^{[36]}$ The increased expression of p70S6k in megakaryocytes of MPN patients might indicate an inhibition of megakaryocyte apoptosis via p70S6k.

More studies were done on assessing the MVD in MPN patients, all show higher MVD in PMF patients compared to ET and PV patients and higher MVD in post-ET myelofibrosis and post-PV myelofibrosis compared to ET and PV indicating that angiogenesis is primarily involved in later stages of the disease. ${ }^{[37-41]}$ We found a correlation between MVD and fibrosis, which is line with the study of Boveri et al. ${ }^{[38]}$, who found the higher the MVD the higher the grading of fibrosis. Higher p70S6k expression in the $\mathrm{mf} 0 / 1$ group compared to the $\mathrm{mf} 2 / 3$ group indicates a declining p70S6k expression with increasing myelofibrosis. This might also explain why we did not find a significant difference in p70S6k expression in bone marrow cells between MPN patients and control patients, while a higher percentage of patients in our study belonged to the $\mathrm{mf} 2 / 3$ group.

In conclusion, the increased cellularity seen in MPN bone marrow might be influenced by the increased expression and anti-apoptotic mechanism of the pErk and pAkt pathway and a decreased expression of the pro-apoptotic protein Bnip3 in bone marrow cells in general. The increased amount of megakaryocytes seen in MPN might be due to the increased pAkt and p70S6k expression. Further, our results also suggest an important pathogenetic role for megakaryocytes in the pathogenesis of MPN patients, mainly in ET patients, as the antiapoptotic pErk, pAkt, p70S6k and Bnip3 expression were all higher expressed in MPN megakaryocytes, mainly in ET patients, compared to the controls. Further, the increased MVD expression in patients with myelofibrosis suggests the important role of angiogenesis in the development of myelofibrosis and therefore is a potential therapeutic target in MPN patients with myelofibrosis. 
Figure 2: Erk and PI3K-Akt signalling pathway.

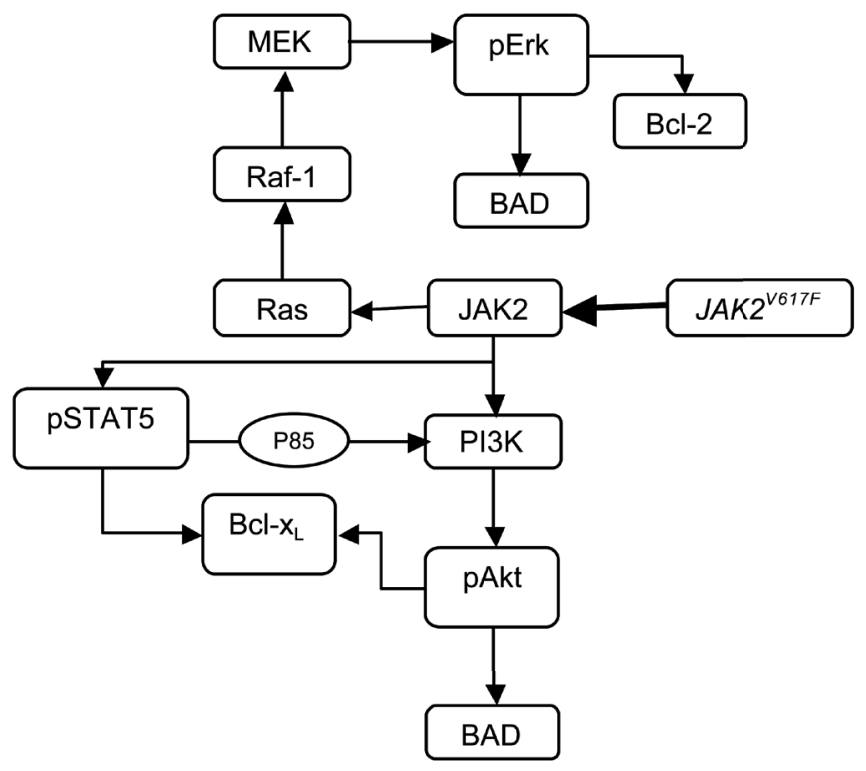

Figure 3: Bnip3 and p70S6k signalling pathway.

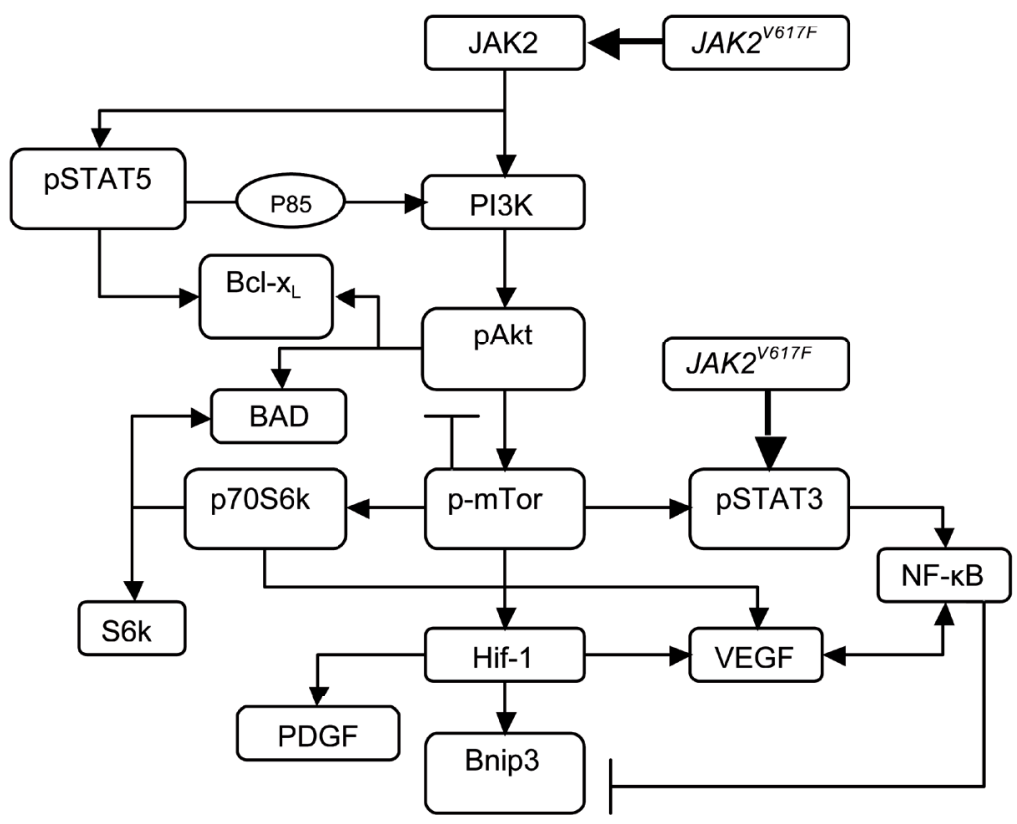




\section{REFERENCES}

1. Vardiman, J.W., N.L. Harris, and R.D. Brunning, The World Health Organization (WHO) classification of the myeloid neoplasms. Blood, 2002. 100(7): p. 2292-302.

2. Campbell, P.J. and A.R. Green, The myeloproliferative disorders. N Engl J Med, 2006. 355(23): p. 2452-66.

3. Murray, J., Myeloproliferative disorders. Clin Med, 2005. 5(4): p. 328-32.

4. Baxter, E.J., et al., Acquired mutation of the tyrosine kinase JAK2 in human myeloproliferative disorders. Lancet, 2005. 365(9464): p. 1054-61.

5. James, C., et al., A unique clonal JAK2 mutation leading to constitutive signalling causes polycythaemia vera. Nature, 2005. 434(7037): p. 1144-8.

6. Kralovics, R., et al., A gain-of-function mutation of JAK2 in myeloproliferative disorders. N Engl J Med, 2005. 352(17): p. 1779-90.

7. Levine, R.L., et al., Activating mutation in the tyrosine kinase JAK2 in polycythemia vera, essential thrombocythemia, and myeloid metaplasia with myelofibrosis. Cancer Cell, 2005. 7(4): p. 387-97.

8. Dhillon, A.S., et al., MAP kinase signalling pathways in cancer. Oncogene, 2007. 26(22): p. 3279-90.

9. Kim, E.K. and E.J. Choi, Pathological roles of MAPK signaling pathways in human diseases. Biochim Biophys Acta. 1802(4): p. 396-405.

10. Boulton, T.G., et al., An insulin-stimulated protein kinase similar to yeast kinases involved in cell cycle control. Science, 1990. 249(4964): p. 64-7.

11. Racke, F.K., et al., Sustained activation of the extracellular signal-regulated kinase/mitogen-activated protein kinase pathway is required for megakaryocytic differentiation of K562 cells. J Biol Chem, 1997. 272(37): p. 23366-70.

12. Rouyez, M.C., et al., Control of thrombopoietin-induced megakaryocytic differentiation by the mitogenactivated protein kinase pathway. Mol Cell Biol, 1997. 17(9): p. 4991-5000.

13. Whalen, A.M., et al., Megakaryocytic differentiation induced by constitutive activation of mitogenactivated protein kinase kinase. Mol Cell Biol, 1997. 17(4): p. 1947-58.

14. Yao, R. and G.M. Cooper, Growth factor-dependent survival of rodent fibroblasts requires phosphatidylinositol 3-kinase but is independent of pp70S6K activity. Oncogene, 1996. 13(2): p. 343-51.

15. Dudek, H., et al., Regulation of neuronal survival by the serine-threonine protein kinase Akt. Science, 1997. 275(5300): p. 661-5.

16. Kauffmann-Zeh, A., et al., Suppression of c-Myc-induced apoptosis by Ras signalling through PI(3)K and PKB. Nature, 1997. 385(6616): p. 544-8.

17. Kennedy, S.G., et al., The PI 3-kinase/Akt signaling pathway delivers an anti-apoptotic signal. Genes Dev, 1997. 11(6): p. 701-13.

18. Kulik, G., A. Klippel, and M.J. Weber, Antiapoptotic signalling by the insulin-like growth factor I receptor, phosphatidylinositol 3-kinase, and Akt. Mol Cell Biol, 1997. 17(3): p. 1595-606.

19. Bakin, A.V., et al., Phosphatidylinositol 3-kinase function is required for transforming growth factor beta-mediated epithelial to mesenchymal transition and cell migration. J Biol Chem, 2000. 275(47): p. 36803-10.

20. Yee, K.W., et al., Phase I/II study of the mammalian target of rapamycin inhibitor everolimus (RAD001) in patients with relapsed or refractory hematologic malignancies. Clin Cancer Res, 2006. 12(17): p. 5165-73.

21. Gonzalez-Garcia, A., et al., A new role for the p85-phosphatidylinositol 3-kinase regulatory subunit linking FRAP to p70 S6 kinase activation. J Biol Chem, 2002. 277(2): p. 1500-8. 
22. Boyd, Adenovirus E1B $19 \mathrm{kDa}$ and Bcl-2 proteins interact with a common set of cellular proteins. Cell, 1994. 79(6): p. 1121.

23. Chen, G., et al., The E1B 19K/Bcl-2-binding protein Nip3 is a dimeric mitochondrial protein that activates apoptosis. J Exp Med, 1997. 186(12): p. 1975-83.

24. Giatromanolaki, A., et al., BNIP3 expression is linked with hypoxia-regulated protein expression and with poor prognosis in non-small cell lung cancer. Clin Cancer Res, 2004. 10(16): p. 5566-71.

25. Swerdlow, S.H., et al., WHO Classification of Tumours of Haematopoietic and Lymphoid Tissues, Fourth Edition. WHO Classification of Tumours, Volume 2. IARC WHO Classification of Tumours, No 2. 2008.

26. Thiele, J., et al., European consensus on grading bone marrow fibrosis and assessment of cellularity. Haematologica, 2005. 90(8): p. 1128-32.

27. Datta, S.R., A. Brunet, and M.E. Greenberg, Cellular survival: a play in three Akts. Genes Dev, 1999. 13(22): p. 2905-27.

28. Datta, S.R., et al., Akt phosphorylation of BAD couples survival signals to the cell-intrinsic death machinery. Cell, 1997. 91(2): p. 231-41.

29. Levine, R.L., et al., Role of JAK2 in the pathogenesis and therapy of myeloproliferative disorders. Nat Rev Cancer, 2007. 7(9): p. 673-83.

30. Kirito, K., et al., Thrombopoietin regulates Bcl-xL gene expression through Stat5 and phosphatidylinositol 3-kinase activation pathways. J Biol Chem, 2002. 277(10): p. 8329-37.

31. Nyga, R., et al., Activated STAT5 proteins induce activation of the PI 3-kinase/Akt and Ras/MAPK pathways via the Gab2 scaffolding adapter. Biochem J, 2005. 390(Pt 1): p. 359-66.

32. Schwaller, J., et al., Stat5 is essential for the myelo- and lymphoproliferative disease induced by TEL/JAK2. Mol Cell, 2000. 6(3): p. 693-704.

33. Liu, F.T. and G.A. Rabinovich, Galectins as modulators of tumour progression. Nat Rev Cancer, 2005. 5(1): p. 29-41.

34. Grimwade, L.F., et al., Phospho-STAT5 and phospho-Akt expression in chronic myeloproliferative neoplasms. Br J Haematol, 2009. 147(4): p. 495-506.

35. Kamishimoto, J., et al., Akt activation through the phosphorylation of erythropoietin receptor at tyrosine 479 is required for myeloproliferative disorder-associated JAK2 V617F mutant-induced cellular transformation. Cell Signal, 2011. 23(5):p. 849-56.

36. Harris, A.L., Hypoxia-a key regulatory factor in tumour growth. Nat Rev Cancer, 2002. 2(1): p. 38-47.

37. Arora, B., et al., Bone marrow angiogenesis and its clinical correlates in myelofibrosis with myeloid metaplasia. Haematologica, 2004. 89(12): p. 1454-8.

38. Boveri, E., et al., Bone marrow microvessel density in chronic myeloproliferative disorders: a study of 115 patients with clinicopathological and molecular correlations. Br J Haematol, 2008. 140(2): p. 162-8.

39. Panteli, K., et al., Angiogenesis in chronic myeloproliferative diseases detected by CD34 expression. Eur J Haematol, 2004. 72(6): p. 410-5.

40. Steurer, M., et al., Increased angiogenesis in chronic idiopathic myelofibrosis: vascular endothelial growth factor as a prominent angiogenic factor. Hum Pathol, 2007. 38(7): p. 1057-64.

41. Gianelli, U., et al., VEGF expression correlates with microvessel density in Philadelphia chromosomenegative chronic myeloproliferative disorders. Am J Clin Pathol, 2007. 128(6): p. 966-73. 


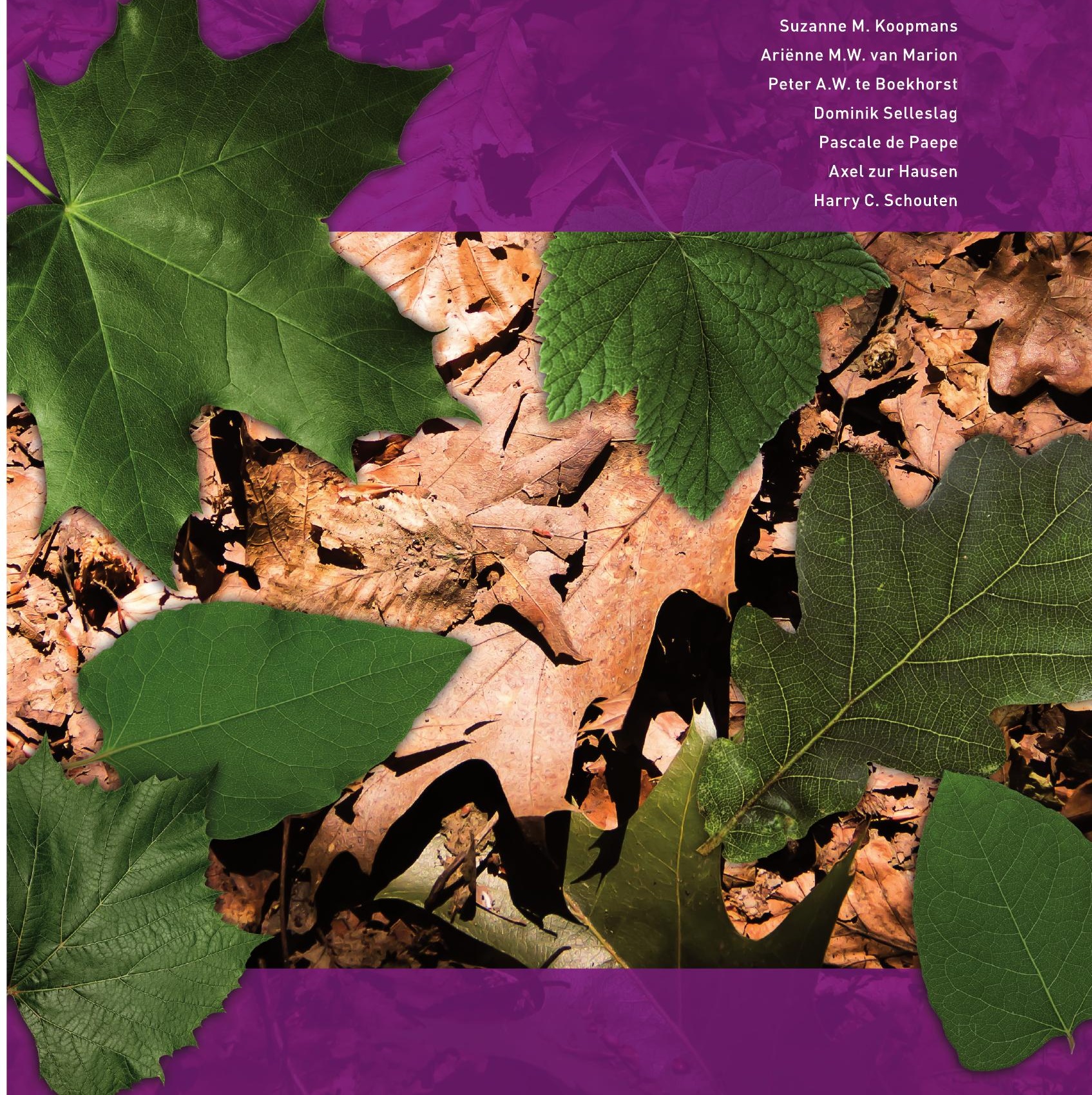




\section{ABSTRACT}

Background: Essential Thrombocythemia (ET), Polycythemia Vera (PV) and Primary Myelofibrosis (PMF) belong to the group of Philadelphia negative Myeloproliferative Neoplasia (Ph-MPN). In the majority of Ph-MPN patients a JAK2 ${ }^{\text {V617F }}$ mutation was suggested to have a causal role in MPN pathogenesis. The JAK2 $2^{\mathrm{V} 17 \mathrm{~F}}$ mutation leads to a sustained activation of JAK2 associated with activation of the mitogen-activated protein kinase (MAPK) signalling pathway, the phosphatidylinositol 3-kinase (PI3K)-Akt signalling pathway and activation of the signal transducer and activator of transcription (STAT) family. The discovery of the JAK2 $2^{\text {V617F }}$ mutation has generated an interest in the JAK/STAT pathway and the effect of JAK2 inhibitors. The aim of this study is to detect the change in presence of galectin-1, galectin-3 and phosphorylated STAT3, STAT5, Erk and Akt in Ph-MPN bone marrow slides before and during treatment with the JAK1/JAK2 inhibitor INCB018424.

Methods: Four patients (one PMF and three post-PV myelofibrosis) treated with INCB018424 and two patients (two PMF) without treatment were analyzed for the expression pattern of galectin-1, galectin-3, pSTAT3, pSTAT5, pErk and pAkt by immunostaining bone marrow biopsy slides before and during treatment. This was followed by automated image analysis. All patients were proven to have the JAK2 ${ }^{\text {V617F }}$ mutation.

Results: The expression of pSTAT3, pSTAT5, pErk and pAkt was reduced in MPN patients during treatment with INCB018424 compared to the expression before treatment. This reduction was absent in the control group of patients.

Conclusion: The results show a possible important role of the JAK2 ${ }^{\text {v617F }}$ mutation in Ph-MPN patients due to the pathological increase in MAPK, PI3K-Akt and STAT signalling pathways. However, considering the preliminary character of the current study a larger study is required to confirm these results. 


\section{INTRODUCTION}

Essential thrombocythemia (ET), polycythemia vera (PV) and primary myelofibrosis (PMF) are the three main Philadelphia chromosome-negative (Ph-) myeloproliferative neoplasia (MPNs). MPNs are a group of clonal haematological diseases, characterised by proliferation of the myeloid, erythroid and/or megakaryocytic cell lineages. ${ }^{[1,2]}$ The development of fibrosis in the bone marrow occurs as a primary disorder in PMF patients or it may follow a preceding disease like $E T$ and $P V{ }^{[3,4]}$ Myelofibrosis is characterised in the peripheral blood by tear-drop erythrocytes, the presence of myeloid precursor cells and megakaryocyte abnormalities with progressive fibrosis of the bone marrow. The symptoms in patients with myelofibrosis consist of splenomegaly, progressive anaemia and constitutional symptoms like fatigue, weight loss, night sweats, bone pain and shortness of breath. Life expectancy for PMF patients vary from 2 to 11 years due to an increased risk of fibrosis and leukemic transformation. Treatment of myelofibrosis is mainly palliative: blood transfusion, splenectomy, radiotherapy and chemotherapy. Stem cell transplantation is the only curative option at the moment. ${ }^{[5]}$ In 2005 the JAK2 $2^{\mathrm{V} 17 F}$ mutation was discovered by several research groups in over $95 \%$ of the PV patients and in approximately half of the ET and PMF patients. The JAK2 ${ }^{\mathrm{V} 617 F}$ results in a constitutive activation of the JAK2 gene. ${ }^{[6-9]}$ The constitutive activation of the JAK2 gene results in an activation of the signal transducer and activator of transcription (STAT) family, the mitogen-activated protein kinase (MAPK) signalling pathway and the phosphatidylinositol 3-kinase (PI3K)-Akt signalling pathway. ${ }^{[7,9]}$ These pathways are involved in angiogenesis, fibrosis and megakaryocyte differentiation and apoptosis. The discovery of this JAK2 $2^{\text {Vil7F }}$ mutation has generated an interest in the development of JAK2 inhibitors. The homozygous $J A K 2^{\mathrm{V} 617 F}$ mutational status has been associated with higher leukocytosis, more pronounced splenomegaly and greater need for cytoreductive therapy in PMF patients. The presence of the $J A K 2^{\text {V617F }}$ mutation, regardless of the homozygous or heterozygous state, has been associated with large splenomegaly, necessity of splenectomy and leukemic transformation. ${ }^{[10]}$ The selective inhibitor for JAK1 and JAK2, INCB018424 (Ruxolitinib), was shown to significantly reduce splenomegaly in patients with myelofibrosis and improved constitutional symptoms. ${ }^{[1]}$ Galectins belong to the family of animal lectins which have been shown to be involved in the development of fibrosis and angiogenesis. ${ }^{[12-14]}$ This has developed an interest in the possible involvement of galectins in MPN development.

In this study, we describe a decrease of phosphorylated STAT3 and STAT5 expression together with a decreased phosphorylation of Erk and Akt in the bone marrow of patients with myelofibrosis and treated with INCB018424. 


\section{MATERIALS AND METHODS}

\section{Study population}

The study was carried out on bone marrow trephines obtained from patients recorded at the University Hospital Maastricht and Erasmus Medical Centre Rotterdam, in the Netherlands, at the Academic Hospital Sint-Jan av Brugge, in Belgium, all between January 2009 and December 2010. The study was approved by the local institutional ethics committee and met the local Tissue Collection criteria. All patients were prior to this study clinically and histological diagnosed according to the 2008 World Health Organization (WHO) classification. [15] The study population consisted of three PMF and three post-PV MF patients ranging from 48 to 79 years (average age of 63.7 years at time of study inclusion). Five patients were male and one female. All six patients carried the JAK2 ${ }^{\mathrm{V} 617 \mathrm{~F}}$ mutation.

Patients were enrolled in an open-label phase III study of the oral JAK1/JAK2 inhibitor INCB018424 versus the best available therapy in patients diagnosed with post-ET MF, postPV MF and PMF according to the 2008 WHO criteria. ${ }^{[15]}$ See the COMFORT-II trial for the details of the study. ${ }^{[16]}$ In our study two patients (both PMF) received besides aspirin no therapy and four patients (one PMF and three post-PV MF) received INCB018424. In the group that was treated with INCB018424 for one year, two patients received $20 \mathrm{mg}$ twice a day lone PMF and one post-PV MF). One patient (post-PV MF) received $20 \mathrm{mg}$ twice a day with a reduction to $5 \mathrm{mg}$ twice a day after one month due to thrombocytopenia. The patient stopped for one month, then received $15 \mathrm{mg}$ twice a day for one month, then stopped for one week again, restarted with $10 \mathrm{mg}$ twice daily for three months and received again $10 \mathrm{mg}$ twice daily. The last patient started with $15 \mathrm{mg}$ twice a day, stopped after three months for one month, due to thrombocytopenia and restarted with $10 \mathrm{mg}$ twice a day.

The grading of fibrosis was done according to the European consensus on grading of bone marrow fibrosis. ${ }^{[17]}$ All patients had a fibrosis grading of three. Bone marrow histology was assessed on the myeloid to erythroid (M:E) ratio, cellularity, number of megakaryocytes and vascular density.

\section{Immunohistochemistry}

The bone marrow biopsy specimens were formalin fixed (a few cases were Bouin fixed), decalcified using the Kristensen procedure for one hour, followed by standard tissue processing and paraffin embedding. From the paraffin-embedded blocks $3 \mu \mathrm{m}$ slices were cut and mounted on starfrost slides (Knitter Gläser, Germany) for immunohistochemical staining. All the antibodies were tested for specificity on positive and negative tumour control slides. They were also individually tested on decalcified control bone marrow biopsies, resulting in a variation of immunohistochemical procedures, optimised for all individual antibodies. 
Antihuman galectin-1 (R\&D systems, Minneapolis, MN) was used with a dilution of 1:500 and antihuman galectin-3 (R\&D systems, Minneapolis, MN) with a dilution of 1:50. After deparaffinization and blocking of endogenous peroxidase activity $\left(0.3 \% \mathrm{H}_{2} \mathrm{O}_{2}\right.$ in methanol) antigen retrieval was performed by boiling in citric acid $(\mathrm{pH} 6)$ for 10 minutes in a water bath of $100^{\circ} \mathrm{C}$. After blocking with $5 \%$ bovine serum albumin/phosphate buffered saline (BSA/ PBS), primary antibodies were applied in 0.5\% BSA/PBS. The slides were then incubated with a biotin-labeled secondary antibody (gal-1: polyclonal swine anti-rabbit, Dako (Glostrup, Denmark) and gal-3: rabbit anti-goat, Dako (Glostrup, Denmark)) with a dilution of 1:200 and 1:500, respectively for 30 minutes. Staining was performed with the StrepABComplex/HRP kit (Dako, Glostrup, Denmark) according to the manufacturer's instructions. The slides were visualized with diaminobenzidine solution (Dako), subsequently counterstained with haematoxylin (Merck, Whitehouse Station, NJ), dehydrated and mounted in Entellan (Merck). Immunohistochemical staining of pSTAT3, pSTAT5, pErk and pAkt was carried out using the antihuman rabbit monoclonal antibody pSTAT3 (Tyr705), pSTAT5 (Tyr694), pErk (Thr202/ Tyr204) and pAkt (Ser473) with a dilution of 1:50, 1:200, 1:100 and 1:25, respectively (Cell signaling Technology, Danvers, MA). After deparaffinization and antigen retrieval by boiling for 20 minutes in $1 \mathrm{mM}$ Tris EDTA pH 8 (pSTAT3 and pSTAT5) or 10mM citric buffer pH 6 (pErk and pAkt) in a water bath of $100^{\circ} \mathrm{C}$, endogenous peroxidase activity was blocked in $3 \% \mathrm{H}_{2} \mathrm{O}_{2}$ in methanol. After treatment with the blocking solution (Tris Buffered Saline Tween (TBST) with $5 \%$ horse serum, pH 7.2-7.6), the primary antibodies were applied in TBST with $5 \%$ horse serum (pSTAT3) or 1\% BSA (pSTAT5, pErk and pAkt) overnight. All slides were then incubated with powervision poly-HRP-Anti Ms/Rb/Ra IgG a histostaining kit (ImmunoLogic, Duiven, the Netherlands) for 40 minutes. Development of the colour and counterstaining was performed as described above.

\section{Analysis of staining}

The staining of gal-1, gal-3, pSTAT3, pSTAT5, pErk and pAkt was analysed using an image processing and analysis system (Leica, Cambridge, UK) linked to a Leica DML3000 light microscope (Leica Quantimet, Germany). QWin (Leica's Windows-based image analysis toolkit-Leica, Cambridge, UKJ was used for postprocessing. The surface area of galectin was measured separately in cell surface and in stroma. All measurements were conducted with a magnification factor of 40 , in at least three to at most five complete bone marrow fields per slide. Total tissue, total area of (cytoplasmic) positive and negative staining (gal-1 and gal-3), total nuclei positive (pSTAT3, pSTAT5, pErk or pAkt) and total nuclei count were recorded. The amount of positivity was calculated as the percentage of the total tissue area lgal-1 and gal3). In practice this was determined as the percentage of positive nuclear pixels relative to the total number of nuclear pixels (pSTAT3, pSTAT5, pErk and pAkt). After analyzing, the mean 
percentage of the three to five fields for gal-1, gal-3, pSTAT3, pSTAT5, pErk and pAkt was calculated.

The author selecting the fields for scoring and the author analysing bone marrow histology were blinded as to the status of the bone marrow.

To validate the data obtained at the molecular level, we tried to isolate DNA from bone marrow biopsies for western blot analysis to confirm our results at RNA level. However, the quality of the DNA was very poor and the DNA was too fragmented to be used.

\section{RESULTS}

Qualitative microscopic evaluation of gal-1 staining was mainly seen in the cytoplasm of endothelial cells as well as in some megakaryocytes, but also stromal cells revealed some background staining. Gal-1 was only partly expressed in precursor erythroid, leukocyte and myeloid cells. Gal-3 was present in endothelial cells, stromal cells and occasionally in megakaryocytes and erythropoietic cells. Phosphorylated STAT3 and STAT5 proteins were localized in the cytoplasm and nuclei of endothelial cells, the nuclei of adipocytes and occasionally more mature erythropoietic cells; precursors of myeloid cells and megakaryocytes. Phosphorylated Erk showed a nuclear expression predominantly in the erythroblasts and occasionally in endothelial and plasma cells. Phosphorylated Akt was expressed in the immature myeloid cell lineage in the cytoplasm as well as nuclear. The megakaryocytes expressed pAkt cytoplasmic.

There was no difference in the gal- 1 and gal- 3 expression between patients treated with

Table 1: Average staining percentages of gal-1, gal-3, pSTAT3, pSTAT5, pErk and pAkt in patients before treatment and after one year of treatment and in controls.

\begin{tabular}{|c|c|c|c|c|}
\hline Staining & $\begin{array}{l}\text { Patients } \\
\text { Before treatment }\end{array}$ & $\begin{array}{l}\text { After } 1 \text { year } \\
\text { treatment }\end{array}$ & $\begin{array}{l}\text { Controls } \\
\text { Before treatment }\end{array}$ & $\begin{array}{l}\text { After } 1 \text { year } \\
\text { treatment }\end{array}$ \\
\hline Gal-1 & 6,71 & 5,78 & 9,38 & 7,01 \\
\hline Gal-3 & 9,79 & 9,93 & 12,01 & 10,48 \\
\hline pSTAT3 & 16,25 & 5,52 & 15,94 & 17,30 \\
\hline pSTAT5 & 6,96 & 2,17 & 12,22 & 17,66 \\
\hline pErk & 21,28 & 11,17 & 24,52 & 22,57 \\
\hline pAkt & 17,00 & 10,84 & 16,75 & 23,48 \\
\hline
\end{tabular}


INCB018424 and the control group. All patients showed a reduction in the expression of gal-1 after one year except for one patient who showed an increased gal-1 expression treated with INCB018424. For gal-3 two patients treated with INCB018424 showed an increased expression after one year (see Figure 1 and Table 1).

The expression of pSTAT3, pSTAT5, pErk and pAkt in the bone marrow of MPN patients showed a considerable reduction during treatment with INCB018424 compared to those who did not receive the treatment (see Figure 1 and Table 1). However, in one patient the expression of pAkt before and during treatment with INCB018424 was nearly the same.

All six patients had a grade three fibrosis before start of the treatment. Neither the control patients nor the patients treated with INCB018424 showed a reduction in the grading of fibrosis. There was a slight decrease in M:E ratio and cellularity compared to the controls. Vascular density was increased in all patients except in one patient who showed a decreased cellularity. The total number of megakaryocytes was decreased in patients treated with INCB018424 compared to the controls, only one patient showed a slight increased total number of megakaryocytes (see Table 2).

Table 2: Details of bone marrow histology before and after one year of treatment for both treated and control patients. pSTAT5, pErk and pAkt in patients before treatment and after one year of treatment and in controls.

\begin{tabular}{|c|c|c|c|c|c|c|c|c|c|c|}
\hline & \multicolumn{2}{|c|}{ M:E ratio } & \multicolumn{2}{|c|}{ Fibrosis grade } & \multicolumn{2}{|c|}{$\begin{array}{c}\text { Megakaryocyte } \\
\text { number per } 5 \mathrm{~mm}^{2}\end{array}$} & \multicolumn{2}{|c|}{$\begin{array}{l}\text { Vascular density } \\
5 \times 1 \mathrm{~mm}^{2}\end{array}$} & \multicolumn{2}{|c|}{$\begin{array}{l}\text { Percentage } \\
\text { cellularity }\end{array}$} \\
\hline & $\begin{array}{c}\text { Before } \\
\text { treat- } \\
\text { ment }\end{array}$ & $\begin{array}{c}\text { After } \\
1 \text { year } \\
\text { treatment }\end{array}$ & $\begin{array}{l}\text { Before } \\
\text { treat- } \\
\text { ment }\end{array}$ & $\begin{array}{c}\text { After } \\
1 \text { year } \\
\text { treatment }\end{array}$ & $\begin{array}{c}\text { Before } \\
\text { treat- } \\
\text { ment }\end{array}$ & $\begin{array}{c}\text { After } \\
1 \text { year } \\
\text { treatment }\end{array}$ & $\begin{array}{c}\text { Before } \\
\text { treat- } \\
\text { ment }\end{array}$ & $\begin{array}{c}\text { After } \\
1 \text { year } \\
\text { treatment }\end{array}$ & $\begin{array}{c}\text { Before } \\
\text { treat- } \\
\text { ment }\end{array}$ & $\begin{array}{c}\text { After } \\
1 \text { year } \\
\text { treatment }\end{array}$ \\
\hline Patient 1 & $3: 1$ & $2: 1$ & 3 & 3 & 147 & 70 & 150 & 90 & 50 & 30 \\
\hline Patient 2 & $2: 1$ & $2: 1$ & 3 & 3 & 120 & 100 & 135 & 261 & 70 & 50 \\
\hline Patient 3 & $4: 1$ & $3: 1$ & 3 & 3 & 85 & 95 & 85 & 180 & 70 & 40 \\
\hline Patient 4 & $3: 1$ & $2: 1$ & 3 & 3 & 580 & 270 & 215 & 305 & 90 & 90 \\
\hline Control 1 & $3: 1$ & $4: 1$ & 3 & 3 & 140 & 260 & 105 & 110 & 70 & 50 \\
\hline Control 2 & $3: 1$ & $3: 1$ & 3 & 3 & 140 & 130 & 90 & 75 & 90 & 90 \\
\hline
\end{tabular}

From one patient (patient number one in the study) it is known that after one year of INCB018424 treatment there was a clinical response; hepato- and splenomegaly disappeared, there was weight gain and haemoglobin increased. This was associated with the decrease in pSTAT3, pSTAT5, pErk and pAkt. 
Figure 1: Percentage of galectin-1 and galectin-3 positivity of total tissue area, percentage of positive nuclear pixels pSTAT3, pSTAT5, pErk and pAkt to total number of nuclear pixels in MPN patients treated with INCB018424 (P1, P2, P3 and P4) and MPN patients without therapy (C1 and C2).

At start of study

$\square$ After one year

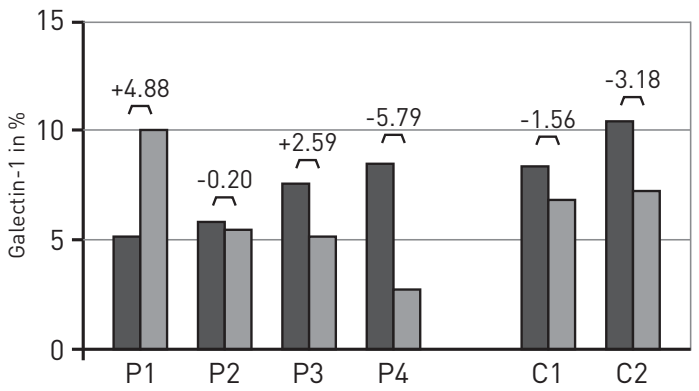

At start of study

$\square$ After one year

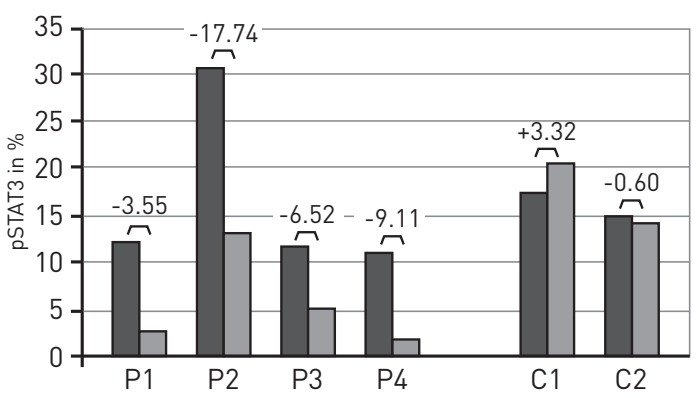

At start of study

$\square$ After one year

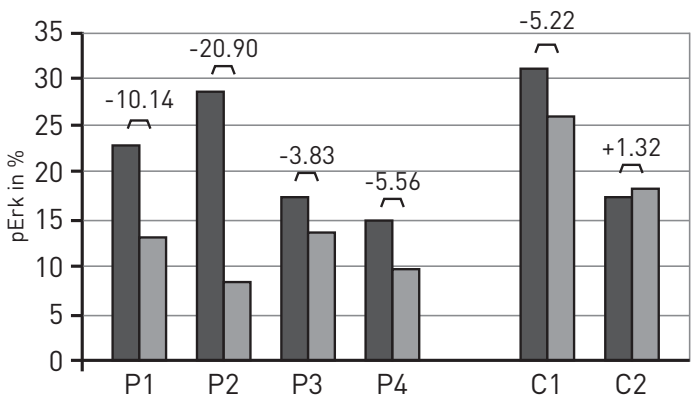

$\square$ At start of study

$\square$ After one year

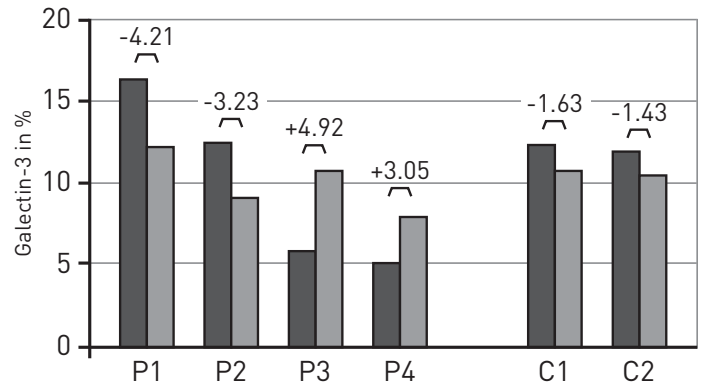

At start of study

$\square$ After one year

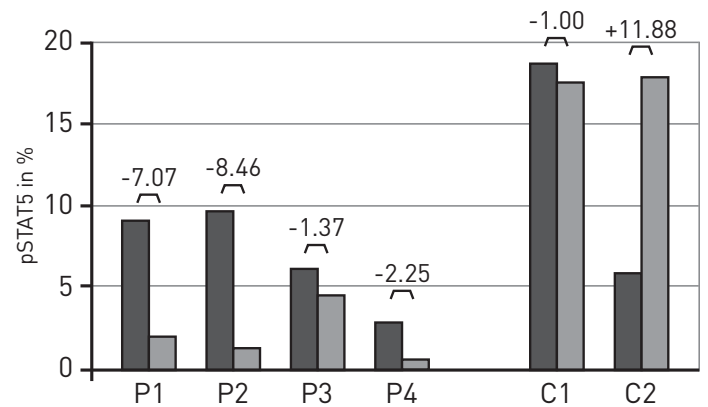

At start of study

$\square$ After one year

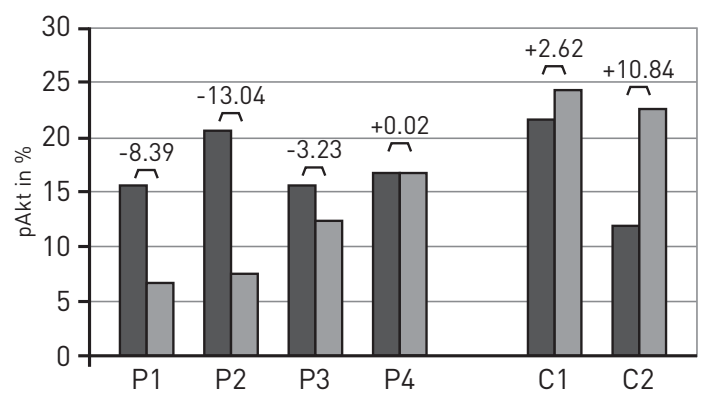




\section{DISCUSSION}

In this preliminary study we examined the expression of gal-1, gal-3, pSTAT3, pSTAT5, pErk and pAkt in post-PV MF and PMF patients treated with INCB018424 versus PMF patients receiving no therapy (see Figure 2). INCB018424 is a selective inhibitor of JAK1 and JAK2 and was shown to have clinical benefits in patients with myelofibrosis. These benefits include reduction of the spleen size, improvement in constitutional symptoms and reduction in the number of red blood cell transfusion-dependent patients. The improvement of clinical symptoms was associated with a decline in levels of circulating inflammatory cytokines such as interleukin-6, tumour necrosis factor and macrophage inflammatory protein $1 .{ }^{[18]}$ Currently, the safest and most effective INCB018424 dose is $15 \mathrm{mg}$ twice-daily. This dose causes minimal non-haematological toxic effects (i.e. diarrhoea, fatigue, headache) and minimal haematological toxic effects such as (new-onset) anaemia and thrombocytopenia. ${ }^{[18]}$ In this preliminary study, the expression of pSTAT3, pSTAT5, pErk and pAkt was reduced in patients treated with INCB018424 compared to patients without therapy. This was not merely a result of decreased cellularity after one year of treatment while the cellularity hardly changed. All the patients in this study were carriers of the JAK2 $2^{\mathrm{V} 677 \mathrm{~F}}$ mutation. It is known that the JAK2 ${ }^{\mathrm{V} 617 F}$ mutation leads to a sustained JAK2 activation resulting in activation of the STAT family, MAPK signalling pathway and PI3K-Akt signalling pathway. ${ }^{[7,9]}$ The JAK1/2 inhibitor INCB018424 might explain the lower pSTAT3, pSTAT5, pErk and pAkt expression in JAK2 ${ }^{\mathrm{V} 617 \mathrm{~F}}$ positive MPN patients treated with INCB018424. This is in line with the study of Quintás-Cardama et al. who found a dose-dependent reduction in the phosphorylated forms of STAT3, STAT5 and Erk in vitro and with the study of Verstovsek et al. who found a dose-dependent suppression of pSTAT3 after INCB018424 treatment. $[18,19]$ Interestingly, the suppression was not only seen in patients with the JAK2 $2^{\text {V617F }}$ mutation but also in patients with wild-type JAK2 suggesting other pathways involved in the JAK activation in MPN patients. ${ }^{[18]}$ Except for the known mutations bone marrow cells, megakaryocytes, stem cells, progenitor cells and myeloid cells also produce aberrant cytokines. These cytokines are produced in JAK2 ${ }^{\mathrm{V} 617 F}$ mutant and non-mutant MPN cells, both activating a signalling in MPNs. In other diseases the STAT3 and NF-signalling have been found to regulate cytokine transcription. ${ }^{[20]}$ Treatment with JAK inhibitors as well as STAT3 deletion can both reduce cytokine expression in MPN cells and thus explain why JAK negative MPN patients can still respond clinically on JAK inhibitor therapy. ${ }^{[21]}$

The complex of the different molecular aberrations together with the production of aberrant cytokine signalling is believed to be the pathogenic mechanism initiating MPN disease. This could explain the effect of JAK inhibitor therapy. ${ }^{[21]}$ 
Figure 2: A. pSTAT3 staining before INCB018424 treatment in patient 2 (HE, 400x), B. pSTAT3 staining after one year of treatment with INCB018424 in patient 2 (HE, 400x), C. pSTAT5 staining before INCB018424 treatment in patient 2 (HE, 400x), D. pSTAT5 staining after one year of treatment with INCB018424 in patient 2 (HE, 400x), E. pErk staining before INCB018424 treatment in patient 2 (HE, 400x), F. pErk staining after one year of treatment with INCB018424 in patient 2 (HE, 400x), G. pAkt staining before INCB018424 treatment in patient 2 (HE, 400x), H. pAkt staining after one year of treatment with INCB018424 in patient 2 (HE, 400x).
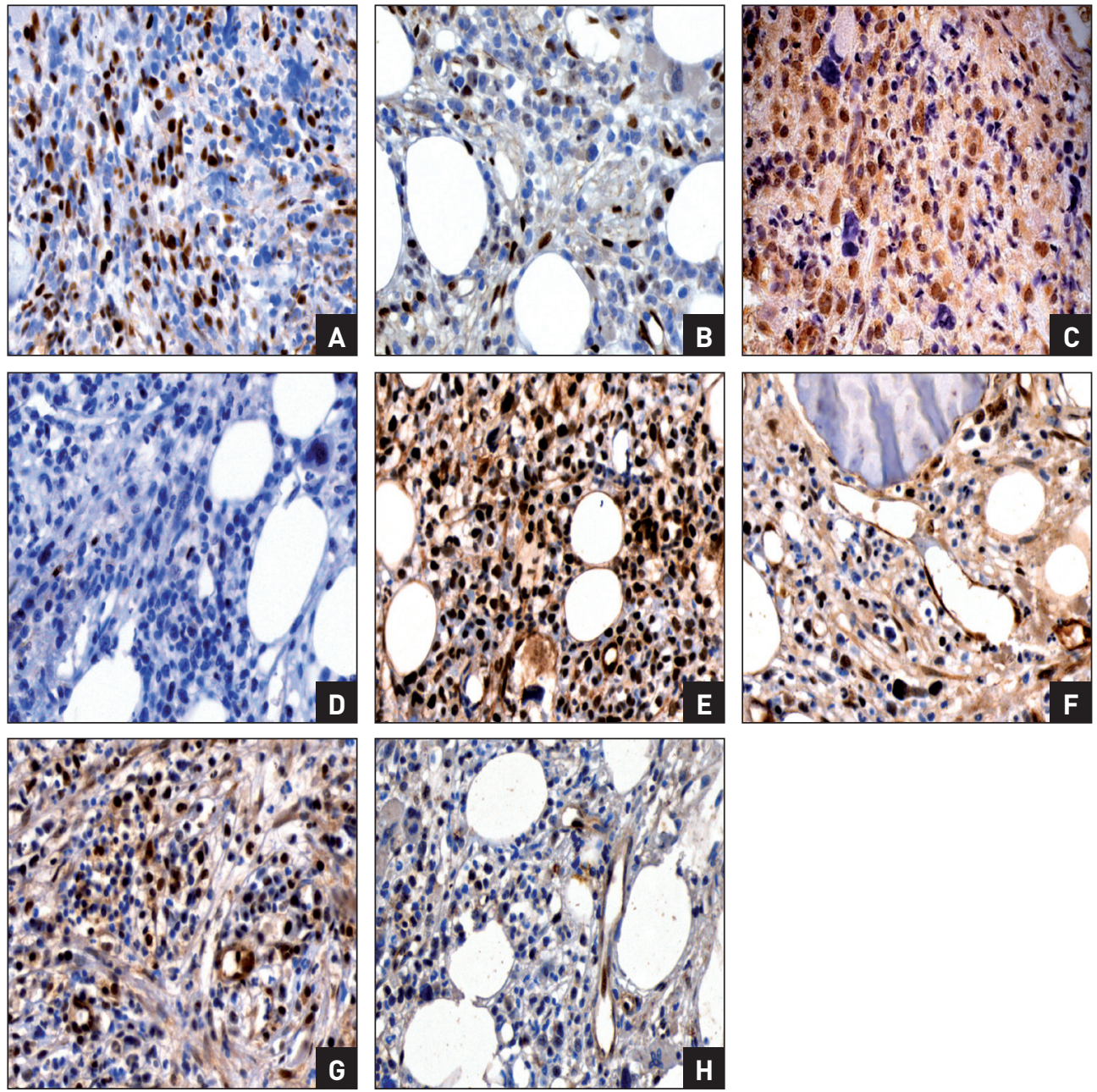
In this preliminary study, patients without therapy showed a lower expression of gal-1 and gal-3 after one year. Three and two patients who were treated with INCB018424 also showed a reduction in the gal-1 and gal-3 expression respectively. This cannot be attributed to a lower cellularity as the cellularity only showed a slight decrease. The binding of gal-1 to $\mathrm{H}$-Ras activates the MAPK lalso known as extracellular signal-regulated kinase (Erk)) pathway ${ }^{[22,23]}$ and the binding of gal-3 to K-Ras modulates the PI3K signalling pathway. ${ }^{[24]}$ This, together with our results, could suggest that signalling pathways activated by gal-1 and gal-3 are not only activated via the JAK2 ${ }^{\mathrm{V} 617 F}$ mutation. This explains why treatment with INCB018424 does not influence the expression of gal-1 and gal-3 in all patients. No reduction in the grading of myelofibrosis was seen in patients treated with INCB018424, which is in line with the study of Verstovsek et al. ${ }^{[18]}$ This remains unsatisfactory, while the development of myelofibrosis determine the worse outcome of patients. A potential effect of long-term treatment and reduction of myelofibrosis is possible but needs further investigation.

In conclusion, we found a lower expression of phosphorylated forms of STAT3, STAT5, Erk and Akt in JAK2 ${ }^{\text {V617F }}$ positive MPN patients after one year of therapy with the JAK1/2 inhibitor INCB018424. Furthermore, this study has shown a lower expression of gal-1 and gal-3 in JAK2 ${ }^{\text {V617F }}$ positive MPN patients, treated and non-treated, which could suggest that other signalling pathways are involved in the expression of gal-1 and gal-3. However, as this is a preliminary study, conclusions cannot be drawn. A larger study is necessary to confirm the results we have found in the study. 


\section{REFERENCES}

1. Campbell, P.J. and A.R. Green, The myeloproliferative disorders. N Engl J Med, 2006. 355(23): p. 2452-66.

2. Murray, J., Myeloproliferative disorders. Clin Med, 2005. 5(4): p. 328-32.

3. Vardiman, J.W., et al., The 2008 revision of the World Health Organization (WHO) classification of myeloid neoplasms and acute leukemia: rationale and important changes. Blood, 2009. 114(5): p. 937-51.

4. Mesa, R.A., et al., Primary myelofibrosis (PMF), post polycythemia vera myelofibrosis (post-PV MF), post essential thrombocythemia myelofibrosis (post-ET MF), blast phase PMF (PMF-BP): Consensus on terminology by the international working group for myelofibrosis research and treatment (IWG-MRT). Leuk Res, 2007. 31(6): p. 737-40.

5. Tefferi, A., Myelofibrosis with myeloid metaplasia. N Engl J Med, 2000. 342(17): p. 1255-65.

6. Baxter, E.J., et al., Acquired mutation of the tyrosine kinase JAK2 in human myeloproliferative disorders. Lancet, 2005. 365(9464): p. 1054-61.

7. James, C., et al., A unique clonal JAK2 mutation leading to constitutive signalling causes polycythaemia vera. Nature, 2005. 434(7037): p. 1144-8.

8. Kralovics, R., et al., A gain-of-function mutation of JAK2 in myeloproliferative disorders. N Engl J Med, 2005. 352(17): p. 1779-90.

9. Levine, R.L., et al., Activating mutation in the tyrosine kinase JAK2 in polycythemia vera, essential thrombocythemia, and myeloid metaplasia with myelofibrosis. Cancer Cell, 2005. 7(4): p. 387-97.

10. Barosi, G., et al., JAK2 V617F mutational status predicts progression to large splenomegaly and leukemic transformation in primary myelofibrosis. Blood, 2007. 110(12): p. 4030-6.

11. Verstovsek, S., et al., The JAK Inhibitor, INCB018424, Demonstrates Durable and Marked Clinical Responses in Primary Myelofibrosis (PMF) and Post-Polycythemia/Essential Thrombocythemia Myelofibrosis (Post PV/ETMF). Blood (ASH Annual Meeting Abstracts), 2008. 112 : Abstract 1762.

12. Hsu, D.K., et al., Galectin-3 expression is induced in cirrhotic liver and hepatocellular carcinoma. Int J Cancer, 1999. 81(4): p. 519-26.

13. Nishi, Y., et al., Role of galectin-3 in human pulmonary fibrosis. Allergol Int, 2007. 56(1): p. 57-65.

14. Thijssen, V.L., et al., Galectin-1 is essential in tumor angiogenesis and is a target for antiangiogenesis therapy. Proc Natl Acad Sci U S A, 2006. 103(43): p. 15975-80.

15. Swerdlow, S.H., et al., WHO Classification of Tumours of Haematopoietic and Lymphoid Tissues, Fourth Edition. WHO Classification of Tumours, Volume 2. IARC WHO Classification of Tumours, No 2. 2008.

16. Harrison,C., et al., JAK inhibition with Ruxolitinib versus best available therapy for myelofibrosis. N Engl J Med, 2012. 366(9): p. 787-98.

17. Thiele, J., et al., European consensus on grading bone marrow fibrosis and assessment of cellularity. Haematologica, 2005. 90(8): p. 1182-32.

18. Verstovsek, S., et al., Safety and efficacy of INCB018424, a JAK1 and JAK2 inhibitor, in myelofibrosis. N Engl J Med, 2010. 363(12): p. 1117-27.

19. Quintas-Cardama, A., et al., Preclinical characterization of the selective JAK1/2 inhibitor INCB018424: therapeutic implications for the treatment of myeloproliferative neoplasms. Blood, 2010. 115(15): p. 310917. 
20. Procu, M., et al., Mutation of the receptor tyrosine phosphatase PTPRC (CD45) in T-cell acute lymphoblastic leukemia. Blood, 2012. 119(19): p.4476-9.

21. Trelinski, J., Robak, T., JAK inhibitors: pharmacology and clinical activity in chronic myeloprolipherative neoplasms. Curr Med Chem, 2013. 20(9): p. 1147-61.

22. Elad-Sfadia, G., et al., Galectin-1 augments Ras activation and diverts Ras signals to Raf-1 at the expense of phosphoinositide 3-kinase. J Biol Chem, 2002. 277(40): p. 37169-75.

23. Javelaud, D. and A. Mauviel, Crosstalk mechanisms between the mitogen-activated protein kinase pathways and Smad signaling downstream of TGF-beta: implications for carcinogenesis. Oncogene, 2005. 24(37): p. 5742-50.

24. Elad-Sfadia, G., et al., Galectin-3 augments K-Ras activation and triggers a Ras signal that attenuates ERK but not phosphoinositide 3-kinase activity. J Biol Chem, 2004. 279(33): p. 34922-30. 

GENERAL DISCUSSION

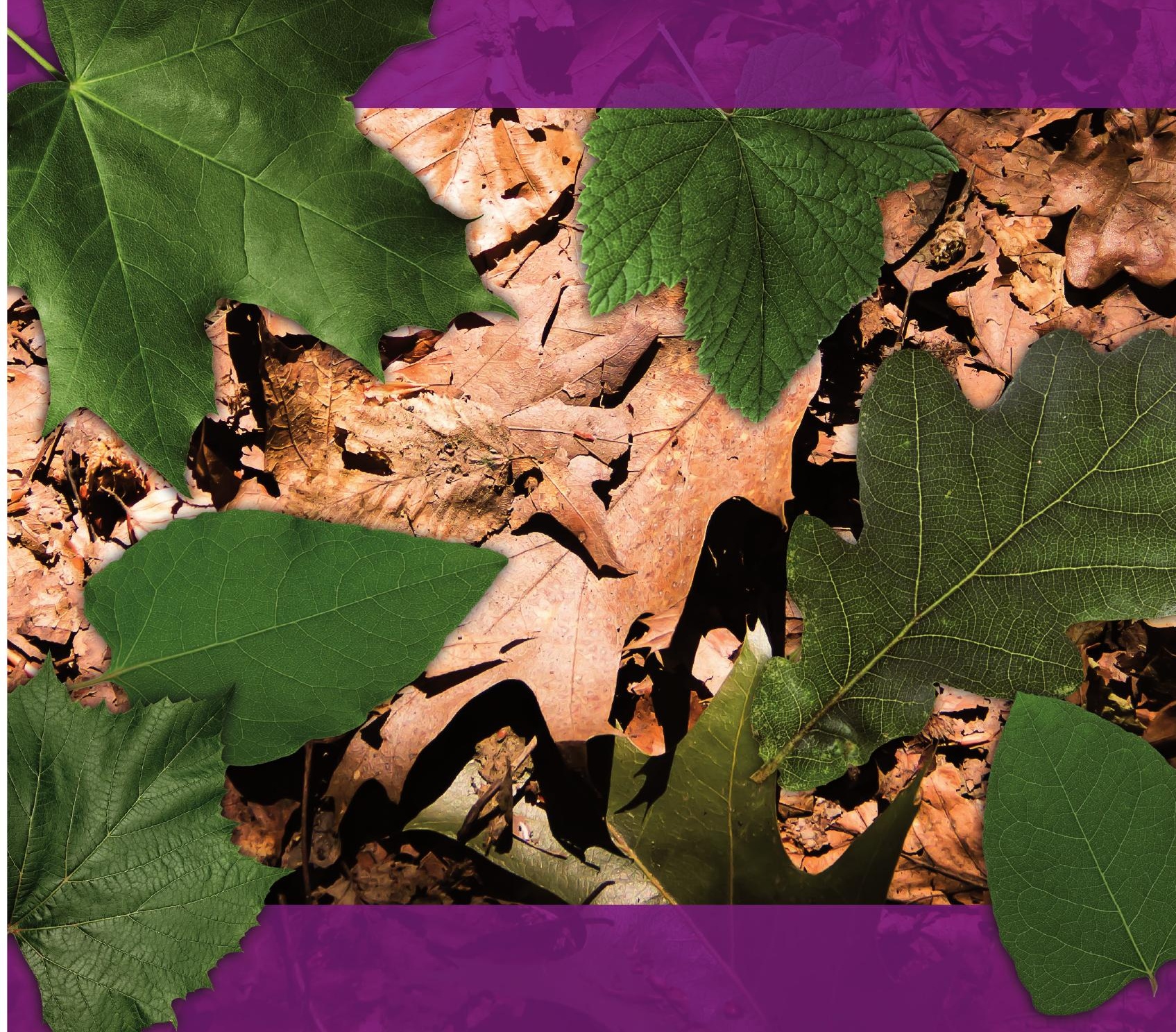




\section{CRITERIA OF MYELOPROLIFERATIVE NEOPLASIA}

Under a microsope, essential thrombocythemia (ET) is recognized by an abnormal proliferation of the megakaryocytic cell line in the bone marrow. Proliferation of all three cell lines and in particular the erythroid cell line can be found in polycythemia vera (PV) patients, whereas proliferation of the megakaryocytic cell line and increasing development of fibrosis in the bone marrow is particular for primary myelofibrosis (PMF) patients. For ET and PV no maturation defects of the blood cells occur; there is only an increased proliferation. In contrast to PMF in which the bone marrow shows abnormal lobulated megakaryocytes of bizarre shapes with maturation defects. ${ }^{[1-4]}$ The WHO classification defines the three distinct Ph-MPN types based on the conjunction of bone marrow morphology, genotype, clinical data and phenotype. However, although it seems clear how to assign the MPN to the appropriate subtype, in practice the distinction between the types is not so straight forward. The initial clinical manifestations are highly variable, show similarities between different MPN entities and can even change with time. Firstly, ET shows phenotypic similarities with the prefibrotic form of PMF. The absence of relevant reticulin fibrosis in bone marrow with dual megakaryocytic and granulocytic myeloproliferation, associated with megakaryocyte dysplasia, is known as prefibrotic myelofibrosis. This new variant has been included as a prodromic phase of PMF into the WHO classification since 2001. ${ }^{[5-7]}$ The phenotype of prefibrotic MF resembles that of ET and are therefore difficult to distinguish from each other. Both entities can be clinically present with thrombocytosis. However, prominent neutrophil proliferation, decreased numbers of erythroid precursors and marked megakaryocyte atypia should distinguish prefibrotic PMF from ET.

Furthermore, erythrocytosis with increased red cell mass is the major feature of PV. ET does not have specific clinical or laboratory features that distinguish it from PV or prefibrotic PMF.

Secondly, ET patients with the JAK2 $2^{\mathrm{V} 17 F}$ mutation show considerable similarities with PV, they have higher haemoglobin and haematocrit levels and a lower blood platelet count. Thirdly, ET and PV can develop into a myelofibrosis form lpost-ET MF and postPV MF respectively) with clinical and laboratory characteristics indistinguishable from PMF. ${ }^{[8-10]}$ Fourthly, the early phases of ET, PV and PMF share many morphological characteristics and are therefore difficult to distinguish from each other when using only histological evaluation (Chapter 3). For this reason, a histological diagnosis should be correlated to the clinical and laboratory features of the patient. In daily practice, however, pathologists receive bone marrow without proper clinical data.

In practice it remains very difficult for clinical doctors as well as for pathologists to assign 
the proper MPN entity. It is thus recommended to establish a panel, similar to the sarcomatoid tumours, lymphoma and melanoma panels, consisting of pathologists and clinical doctors to generate more expertise in the field of MPNs. The question remains however, whether it is clinically relevant to distinguish the different MPN entities.

The discovery of the JAK2 $2^{\mathrm{V} 617 F}$ mutation in more than $95 \%$ of the PV patients and in approximately $50 \%$ of ET and PMF patients ${ }^{[11-14]}$ is of paramount importance. Unfortunately, it has not changed the ability to discriminate between the different MPN entities.

While ET, PV and PMF show overlap in their morphological characteristics, clinical signs and symptoms and possibly even share the same molecular mutation (JAK2 ${ }^{\mathrm{V} 617 F}$ ), questions are raised whether ET, PV and PMF are indeed three separate entities or just different manifestations of the same disorder? It is advised to classify MPN patients in two groups according to the presence or absence of the JAK2 $2^{\text {V617F }}$ mutation. Both groups have a different prognosis of disease progression and survival chances (Chapter 1 and 2). Regarding post-ET MF, post-PV MF and PMF, there are no differences in survival chance, clinical and laboratory features and treatment? The relevance of distinguishing these types is therefore questionable. It is suggested to use the term MPN-associated myelofibrosis (MPN-MF) to encompass these disorders instead. ${ }^{[8]}$

\section{FIBROSIS}

Myelofibrosis (MF) is the existence of fibrosis in the bone marrow and is determined by the amount of reticulin staining. Myelofibrosis can occur as primary disorder in PMF but can also develop in the setting of ET and PV referred to as post-ET MF and post-PV MF respectively. The histologic grading of fibrosis in the bone marrow shows an acceptable inter observer variability as we have shown in Chapter 3. Clinically, the development of fibrosis in PMF, ET and PV patients indicates poor survival chances. ${ }^{[15]}$ So far myelofibrosis does not respond to any of the available therapies. Even the promising JAK2 inhibitors do not reverse the development of bone marrow fibrosis, although our preliminary data suggest that the fibrosis stays stable during JAK inhibitor treatment (Chapter 6). However, it might be too early to draw conclusions from the present JAK2 inhibitor studies, because the treatment duration and follow-up period were very short. Finding a therapy that can cure or at least reduce the bone marrow fibrosis is important, as the development of fibrosis is a very important marker indicating a severe worsening of the patients' condition. The underlying pathogenesis of fibrosis development is still unclear and should be clarified before new therapies for 
myelofibrosis can be developed. Allogeneic stem cell transplantation is the only curative option at the moment.

\section{MICROVESSEL DENSITY}

Angiogenesis is the formation of new capillaries and vessels from existing blood vessels and plays an important role in malignancies. The microvessel density (MVD) is a good morphological surrogate for angiogenesis in bone marrow. In our study we investigated the MVD and estimated the number of vessels in MPN bone marrow with the MVD 'hotspot' method. However, the MVD 'hotspot' method might not be the best way to estimate the number of vessels in $\mathrm{Ph}-\mathrm{MPN}$ bone marrow. Bone marrow of $\mathrm{Ph}-$ MPN shows a broad variation of cellularity and, therefore, the amount of vessels can be underestimated in marrows with a relative low cellularity. This is especially true when comparing them to marrows with higher cellularity. This is due to the fact that with the 'hotspot' method there is no correction of the absolute number of vessels for the cellularity of the specimen. A solution might be to use an index that accounts for both the absolute marrow cellularity and the percentage of immuno-stained cells. On the other hand, however, the use of the MVD 'hotspot' method allows us to easily compare results to previous data and to the majority of the relevant studies in the literature (Chapter 4 and 5).

We have also tried to stain MPN bone marrow trephines with vascular endothelial growth factor (VEGF), although this seemed not reliable in our hands; the VEGF staining in patients from two different hospitals showed too much background staining or was stained so weakly that we have excluded it from the analysis. At the same time, the VEGF staining in another series of patients from a third hospital showed promising results: almost no background staining was visible and a good differentiation between staining cells and non-staining cells was visible (Chapter 4).

There is discussion whether MVD or VEGF positively correlates with the degree of fibrosis. MPN patients with increasing development of fibrosis show also increasing MVD, but it is unclear in which order symptoms are appearing; increased angiogenesis which contributes to the increasing development of fibrosis or increased fibrosis which contributes to the increased angiogenesis. It is known that galectin-1 is involved in angiogenesis. Galectin-1 has been shown to be increased in the endothelium of tumours and to be the target for anginex. This is a novel artificial cytokine-like peptide inhibiting angiogenesis, which binds to galectin-1 resulting in inhibition of angiogenesis. ${ }^{[16]}$ In Chapter 4 we have described an increased expression of galectin-1 along with an 
increased MVD in MPN patients, suggesting a possible role for anginex as angiogenesis inhibitor in MPN patients (Chapter 4).

\section{DECALCIFICATION PROBLEMS}

Immunohistochemical analysis of the bone marrow is a very important procedure in the diagnosis of haematological disorders, because differentiation by means of conventional histological techniques is often insufficient. The advantage of the use of sections of trephine bone biopsies is the preservation of tissue architecture and the relationship between haematopoietic tissue and bone trabeculae. However, one of the problems of immunohistochemical analyses is the fixation procedure and especially the decalcification procedures. Several laboratories in Europe still use the acid decalcification procedure. This hampers immunohistochemistry and the extraction of DNA from paraffin embedded bone marrow biopsies, while the acid completely destroys DNA for genetical analyses. We have tried to extract DNA from paraffin embedded and acid as well as EDTA decalcified bone marrow biopsies to determine the JAK2 status from patients who had already past away prior the availability of the JAK2 test. Unfortunately, in most cases the quality of the DNA was very poor and DNA was too fragmented to be able to use the JAK2 test (Chapter 4 and 5).

It is therefore recommended to develop a European guideline for the fixation and especially decalcification procedure to be used in every European laboratory. This makes it possible to compare several stainings done by different laboratories.

\section{STAININGS}

Another problem with the current practice of immunohistochemistry is the impossibility to prove whether the staining corresponds to the affected protein. To resolve this problem it is recommended to validate the primary antibody with a western blot. An additional disadvantage of immunohistochemistry is the unreliable quantification of the staining. Immunohistochemical staining can be present in the membrane, cytoplasm, nucleus and/or stroma. Assessment of this staining can range from being present or absent and increased or decreased expression. The assessment of nuclear stained cells might be more reliable than assessment of cytoplasmic stained cells or stroma, as the latter two are less specific than nuclear staining and are more sensitive to reactivity. There are also different scoring systems, mainly manually and therefore 
dependent on the experience and alertness of the interpreter. This has been improved since the use of computerized image analysis systems.

On the other hand, immunohistochemistry also has several advantages: 1. the staining localizes exactly where the component is located in the tissue; 2 . it is possible to recognize a certain pattern in the tissue and what the relationship is between other cells; 3 . it is a very quick method and can give results in only one day and 4. tissue staining is also a method for detecting the tissue sensitivity for certain therapies by means of detecting the presence or increased amount of a certain molecular target. In conclusion, the use of immunohistochemistry is a useful and quick method in the procedure of diagnosing haematological disorders, but might be more reliable if there is a European fixation and decalcification guideline and if standardized protocols for assessment will be available.

\section{THE JAK2 $2^{\text {V617F }}$ MUTATION}

Although progress in the understanding has been made since the discovery of the $J A K 2^{\text {V617F }}$ mutation, the underlying pathogenetic cause(s) of MPN has not been elucidated completely. It is known that the JAK-STAT pathway, Erk and PI3K-Akt pathway play a major role, mainly driven by the JAK2 ${ }^{\mathrm{V} 617 F}$ mutation, with an increased phosphorylation of STAT3, STAT5, Erk and Akt in MPN patients. ${ }^{[12,17]}$

Although the JAK2 ${ }^{\mathrm{V} 617 F}$ mutation explains many of the clinical and laboratory characteristics of ET, PV and PMF, it is not the initiating mutation (Chapter 1 and 2).

How one mutation can be responsible for three different clinical phenotypes is still an unresolved issue. At the moment there are three hypotheses. The first hypothesis is the 'gene-dosage' hypothesis, which postulates a correlation between phenotype and allele burden, which is the ratio between mutant and wild type JAK2 in haematopoietic cells. Most PV patients are homozygous for the JAK2 V617F mutation, while ET patients are heterozygous. Some ET patients with increasing allele burden develop PV or PMF over time. Importantly, ET patients with the JAK2 $2^{\text {V617F }}$ mutation have a "PV-like" phenotype compared to ET patients who do not carry the mutation. However, the 'genedosage' does not entirely explain the phenotypic heterogeneity of the MPNs. Differences in mice with different genetic background have been observed leading to a second hypothesis of 'host genetic factors'. This hypothesis postulates that host genetic characteristics might act as modifiers of the phenotype in combination with the $J A K 2^{\text {V617F }}$ mutation, for instance, singe nucleotide polymorphisms (SNPs). Finally, a third hypothesis is the 'pre-JAK2 phase' which postulates additional somatic mutations 
or inherited predisposing alleles present before the JAK2 $2^{\text {V617F }}$ mutation, which are responsible for the clonal haematopoiesis and determine the phenotype. This preJAK2 phase might even be responsible for generating the JAK2 ${ }^{\mathrm{V} 617 F}$ mutation or might act synergistically. ${ }^{[18,19]}$

The discovery of the JAK2 $2^{\mathrm{V} 617 F}$ mutation has generated an interest in the development of JAK inhibitors. The results are very promising with great reduction in splenomegaly and improvement of constitutional symptoms. Some JAK inhibitors reduce the levels of circulating inflammatory cytokines and the JAK2 $2^{\text {V617F }}$ allele burden. The JAK inhibitors also reduce the phosphorylation of STAT3, STAT5, Erk and Akt (Chapter 6). Although the quality of life of MPN patients is greatly improved by JAK inhibitors, they only generate a stable disease situation; they do not cure the patients. The JAK inhibitors inhibit not only the mutated JAK2 gene, but also the normal JAK2 gene. While JAK2 is important for the normal blood cell production, a balance should be found between enough JAK inhibition and too much inhibition which can be lethal. Furthermore, it is remarkable that not only patients positive for the JAK2 $2^{\mathrm{V} 617 F}$ mutation benefit from the JAK inhibitors, but also patients who do not carry the JAK2 ${ }^{\text {V617F }}$ mutation. ${ }^{[20]}$ This indicates another mechanism involved in the MPNs with increased activation of the JAK-STAT pathway. The question remains however, whether the search for new mutations enlighten the pathogenesis of MPN. None of the discovered mutations so far have turned out to be the critical driver of the underlying myeloproliferative process. It seems that the known mutations are secondary mutations. Therefore from a therapeutical point of view, it is more reasonable to investigate the JAK-STAT pathway.

Indeed, bone marrow cells also produce aberrant cytokines which are produced in $J A K 2^{V 617 F}$ mutant and non-mutant MPN cells. The cytokines activate a signalling in MPNs. STAT3 and NF- $K \beta$ signalling have been found to regulate cytokine transcription in other diseases. ${ }^{[21]}$ Treatment with JAK inhibitors and STAT3 deletion can both reduce the expression of cytokines in the chronic inflammatory bone marrow and resolve the issue why JAK negative MPN patients also respond clinically to JAK inhibitor therapy. ${ }^{[22]}$ Indeed, the complex of the different molecular aberrations together with the production of aberrant cytokine signalling is believed to be the pathogenic mechanism initiating MPN disease and explaining the effect of JAK inhibitor therapy. ${ }^{[21]}$

Pomalidomide, an immunomodulating drug, could also be a promising drug in the treatment of MPN. Pomalidomide is structurally related to lenalidomide and thalidomide, but is less toxic. It is believed that the abnormal cytokine environment in myelofibrosis contributes to anaemia, constitutional symptoms, cachexia and extramedullary haematopoiesis. Due to its anti-angiogenic, anti-cytokine and pro- 
erythropoietic properties, pomalidomide has been shown to be effective in the treatment of myelofibrosis. ${ }^{[23]}$ However, ongoing phase III studies need to confirm these results.

In this thesis we decided to focus on the JAK-STAT pathway and the Erk, PI3K/Akt pathway. Other interesting pathways to look at in the future are the proteins that inhibit STAT3, STAT5, JAK2, PI3K, Akt and Erk, like PIAS, GATA-1/2, SOCS, SHP and pTEN. Also the epigenetic changes in these pathways might be very interesting to investigate. Furthermore, the MPNs are characterized by the continuous release of inflammation products from activated leukocytes and platelets associated with a chronic inflammation. Chronic inflammation is also associated with an increase in DNA methylation. If chronic inflammation has a major impact on DNA-methylation in MPNs it may be hypothesized that a chronic inflammatory bone marrow microenvironment with an increase in cytokines and chemokines result in epigenetic changes, genomic instability and DNA mutations in haematopoietic cells. This may subsequently initiate clonal development and also drive clonal evolution by triggering additional mutations. This could further enhance clonal expansion and release of inflammatory products. A growing list of mutations has been generated during the last couple of years. However, it is questionable whether this has generated more insight in the pathogenesis of MPN development. The last couple of years it has become more clear that the MPNs are not a single-hit disease, but that the nature of these diseases is more complex. The JAK2 ${ }^{\mathrm{V} 617 F}$ mutation has turned out not to be the initiating mutation because only half of the PMF and ET patients carry the JAK2 wild type gene. The existence of a preJAK2 phase has become more likely, with EZH2, TET2 and ASXL1 as early events preceding the JAK2 ${ }^{\mathrm{V} 617 F}$ mutation. However, the influence of genetic and environmental changes play also a role and act as modifiers in combination with the JAK2 ${ }^{\text {V617F }}$ mutation.

The EZH2, TET2 and ASXL1 mutations may also occur late during disease progression. This indicates that the occurrence of these mutations, regardless of the order of appearance, drives disease progression while these mutations are more frequent in PMF patients. Mutations which are more frequent seen in disease transformation are IDH1/2 and TP53 mutations. ${ }^{[24,25]}$

Thus, some mutations are more involved in the early phases of MPN in combination with the JAK2 $2^{V 617 F}$ mutation, other environmental and genetic changes which can lead to disease progression and transformation over time with the occurrence of other mutations.

However, very recently another mutation has been discovered, the calreticulin (CALR) mutation. The CALR mutation was predominantly found in patients with ET and PMF 
without the JAK2 $2^{\mathrm{V} 617 F}$ or MPL mutation, in approximately $70 \%$ of patients. This mutation also activates the JAK/STAT pathway. ET patients with the CALR mutation had higher platelet counts, lower haemoglobin and lower white cell count. PMF patients with the CALR mutation had lower white cell count and higher platelet count. The lower white cell count and lower haemoglobin level might be responsible for the observed lower frequency of thrombosis in MPN patients with a CALR mutation. Patients with the $C A L R$ mutation were shown to have longer overall survival and have a more benign clinical course than MPN patients with the JAK2 ${ }^{\text {v617F }}$ or MPL mutation.

The discovery of another mutation in approximately $70 \%$ of MPN patients without $J A K 2^{\text {V617F }}$ or MPL mutation might suggest that MPN patients can be divided in MPN patients with the JAK2 $2^{\text {V617F }}$ or MPL mutation and MPN patients with the CALR mutation, with patients with the CALR mutation having a longer overall survival and lower frequency of thrombosis. ${ }^{[26,27]}$ 


\section{REFERENCES}

1. Campbell, P.J. and A.R. Green, The myeloproliferative disorders. N Engl J Med, 2006. 355(23): p. 2452-66.

2. Michiels, J.J., et al., Current diagnostic criteria for the chronic myeloproliferative disorders (MPD) essential thrombocythemia (ET), polycythemia vera (PV) and chronic idiopathic myelofibrosis (CIMF). Pathol Biol (Paris), 2007. 55(2): p. 92-104.

3. Murray, J., Myeloproliferative disorders. Clin Med, 2005. 5(4): p. 328-32.

4. Swerdlow, S.H., et al., WHO Classification of Tumours of Haematopoietic and Lymphoid Tissues, Fourth Edition. WHO Classification of Tumours, Volume 2. IARC WHO Classification of Tumours, No 2. 2008.

5. Thiele, J., et al., Idiopathic primary osteo-myelofibrosis: a clinico-pathological study on 208 patients with special emphasis on evolution of disease features, differentiation from essential thrombocythemia and variables of prognostic impact. Leuk Lymphoma, 1996. 22(3-4): p. 303-317.

6. Thiele, J., et al., Clinicopathological diagnosis and differential criteria of thrombocythemias in various myeloproliferative disorders by histopathology, histochemistry and immunostaining from bone marrow biopsies. Leuk Lymphoma, 1999. 33(3-4): p.207-218.

7. Thiele, J, . et al., Initial (prefibrotic) stages of idiopathic (primary) myelofibrosis (IMF) - a clinicopathological study. Leukemia, 1999. 13(11): p.1741-1748.

8. Spivak, J.L. and R.T. Silver, The revised World Health Organization diagnostic criteria for polycythemia vera, essential thrombocytosis, and primary myelofibrosis: an alternative proposal. Blood, 2008. 112(2): p. 231-9.

9. Mesa, R.A., et al., MPN-associated myelofibrosis (MPN-MF). Leuk Res. 35(1): p. 12-3.

10. Vardiman, J.W., N.L. Harris, and R.D. Brunning, The World Health Organization (WHO) classification of the myeloid neoplasms. Blood, 2002. 100(7): p. 2292-302.

11. Baxter, E.J., et al., Acquired mutation of the tyrosine kinase JAK2 in human myeloproliferative disorders. Lancet, 2005. 365(9464): p. 1054-61.

12. James, C., et al., A unique clonal JAK2 mutation leading to constitutive signalling causes polycythaemia vera. Nature, 2005. 434(7037): p. 1144-8.

13. Kralovics, R., et al., A gain-of-function mutation of JAK2 in myeloproliferative disorders. N Engl J Med, 2005. 352(17): p. 1779-90.

14. Levine, R.L., et al., Activating mutation in the tyrosine kinase JAK2 in polycythemia vera, essential thrombocythemia, and myeloid metaplasia with myelofibrosis. Cancer Cell, 2005. 7(4): p. 387-97.

15. Mesa, R.A., et al., Primary myelofibrosis (PMF), post polycythemia vera myelofibrosis (post-PV MF), post essential thrombocythemia myelofibrosis (post-ET MF), blast phase PMF (PMF-BP): Consensus on terminology by the international working group for myelofibrosis research and treatment (IWG-MRT). Leuk Res, 2007. 31(6): p. 737-40.

16. Thijssen, V.L., et al., Galectin-1 is essential in tumor angiogenesis and is a target for antiangiogenesis therapy. Proc Natl Acad Sci U S A, 2006. 103(43): p. 15975-80.

17. Levine, R.L. and D.G. Gilliland, JAK-2 mutations and their relevance to myeloproliferative disease. Curr Opin Hematol, 2007. 14(1): p. 43-7.

18. Passamonti, F. and E. Rumi, Clinical relevance of JAK2 (V617F) mutant allele burden. Haematologica, 2009. 94(1): p. 7-10.

19. Vannucchi, A.M., et al., Clinical correlates of JAK2 $2^{\mathrm{V} 617 \mathrm{~F}}$ presence or allele burden in myeloproliferative neoplasms: a critical reappraisal. Leukemia, 2008. 22(7): p. 1299-307. 
20. Stein, B.L., J.D. Crispino, and A.R. Moliterno, Janus kinase inhibitors: an update on the progress and promise of targeted therapy in the myeloproliferative neoplasms. Curr Opin Oncol. 23(6): p. 609-16.

21. Procu, M., et al., Mutation of the receptor tyrosine phosphatase PTPRC (CD45) in T-cell acute lymphoblastic leukemia. Blood, 2012. 119(19): p.4476-9.

22. Trelinski, J., Robak, T., JAK inhibitors: pharmacology and clinical activity in chronic myeloprolipherative neoplasms. Curr Med Chem, 2013. 20(9): p. 1147-61.

23. Tefferi, A., et al., Pomalidomide is active in the treatment of anemia associated with myelofibrosis. J Clin Oncol 2009. 27(27): p. 4563-9.

24. Tefferi, A., Novel mutations and their functional and clinical relevance in myeloproliferative neoplasms: JAK2, MPL, TET2, ASXL1, CBL, IDH and IKZF1. Leukemia 2010. 24(6): p. 1128-38.

25. Vainchenker, W., et al., New mutations and pathogenesis of myeloproliferative neoplasms. Blood 2011. 118(7): p.1723-35.

26. Klampfl, T., et al., Somatic mutations of calreticulin in myeloproliferative neoplasms. $N$ Engl J Med 2013. 369(25): p. 2379-90.

27. Nangalia, J., et al., Somatic CALR mutations in myeloproliferative neoplasms with nonmutated JAK2. N Engl J Med 2013. 369(25): p. 2391-405. 



\section{SUMMARY / SAMENVATTING}

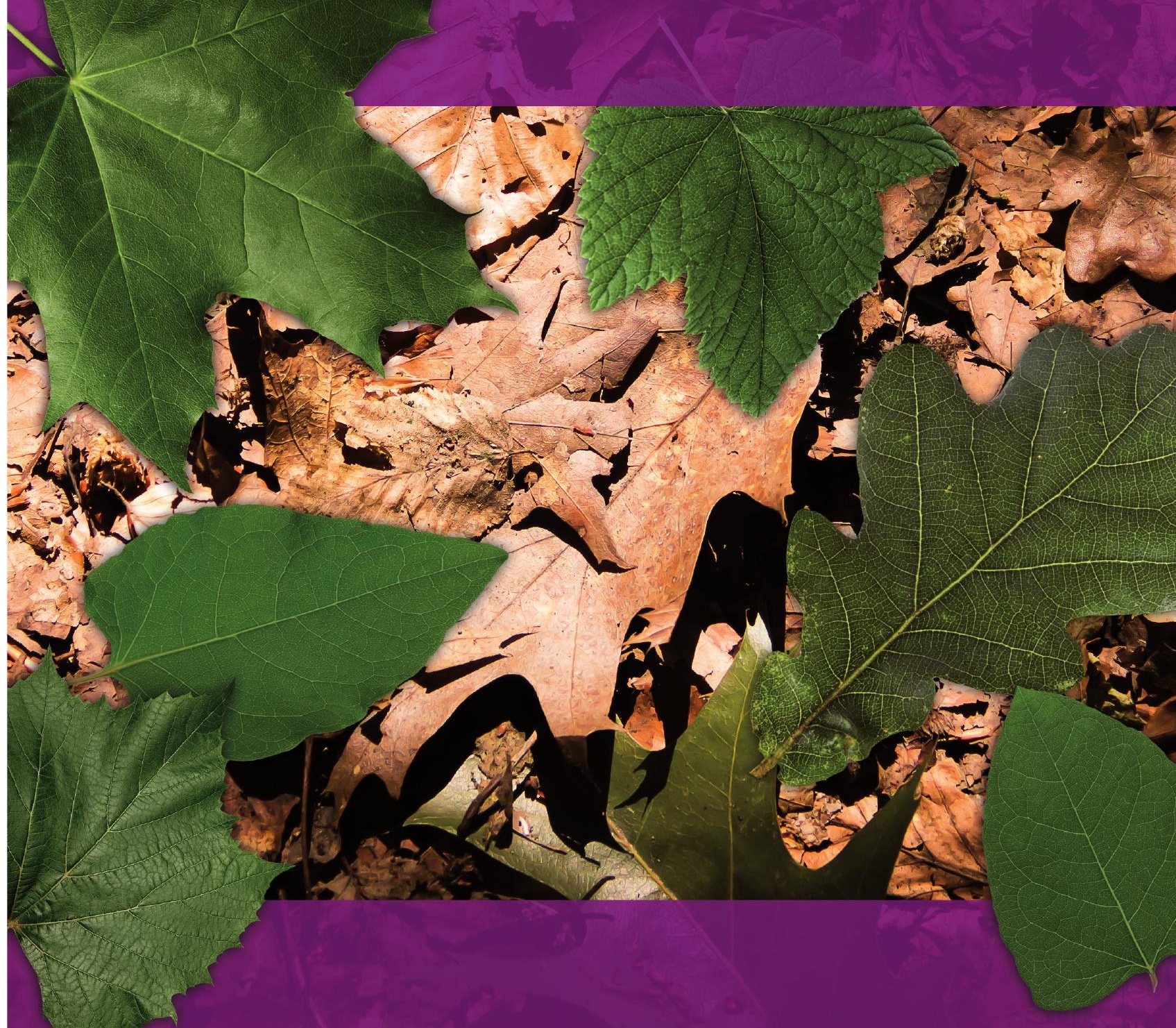




\section{SUMMARY}

Myeloproliferative neoplasia (MPN) are clonal bone marrow stem cell disorders characterized by proliferation of the erythroid, megakaryocytic and/or myeloid cell lines. Essential thrombocythemia (ET), polycythemia vera (PV) and primary myelofibrosis (PMF) belong to the Philadelphia chromosome negative MPNs.

In the last couple of years it has become evident that bone marrow morphology is a cornerstone of MPN diagnosis and disease classification.

Together with the discovery of the JAK2 ${ }^{\mathrm{V} 617 \mathrm{~F}}$ mutation more insight in the development of MPN has been generated.

In chapter 1 and 2 we describe the clinical and histological criteria of MPN and the symptoms patients with a MPN display. The symptoms of the different MPN entities show a great overlap which makes it difficult to distinguish these three entities clinically from each other. Bone marrow pathology is an important type of investigation for a definitive diagnosis. Bone marrow in ET patients is predominantly characterized by a megakaryocyte proliferation, lying in loose clusters. PV shows a trilinear proliferation in the bone marrow, but the erythroid cell line is predominantly present. Bone marrow in PMF patients shows reticulin fibrosis and proliferation of the megakaryocytes which are clustered together very tightly. However, ET as well as PV patients can show some reticulin fibrosis in their bone marrow, known as post-ET myelofibrosis (post-ET MF) and post-PV myelofibrosis (post-PV MF) respectively.

The presence of the JAK2 $2^{\text {V617F }}$ mutation in all MPN entities makes it more difficult to differentiate between ET, PV and PMF. The JAK2 ${ }^{\mathrm{V} 617 \mathrm{~F}}$ mutation is present in approximately $95 \%$ of PV patients and in approximately $50 \%$ in ET and PMF patients. The morphological and clinical overlap and the presence of the JAK2 ${ }^{\mathrm{V} 617 F}$ mutation in MPN patients causes ambiguity whether ET, PV and PMF are three distinct diseases or simply expressions of one disease. We propose to divide the MPNs in JAK2 positive MPNs and JAK2 negative MPNs with a subdivision in patients with and without myelofibrosis.

Furthermore, we give a brief overview of the treatment of MPN patients. The treatment of the individual MPNs depends on the risk category. ET and PV patients belonging to the low and intermediate risk group are treated with a low dose of aspirin. PV patients will also receive phlebotomie. In ET and PV patients belonging to the high risk group a chemotherapeutical is added. In PMF patients belonging to the low and intermediate-1 risk category a 'wait-and-see' approach is justified. The intermediate-2 and high risk group receives chemotherapy or will be allocated for an allogeneic stemcell transplantation or experimental drugs. 
In chapter 3 we assessed the reproducibility of the current major individual morphological characteristics described in the WHO classification and the reproducibility of the histological diagnosis. The discovery of the JAK2 ${ }^{\mathrm{V} 617 F}$ mutation resulted in a revision of the WHO diagnostic criteria of the MPN in 2008. However, there is concern of the reproducibility of morphological features as stated in these criteria. This was also shown in our study; we show a high degree of consensus for the individual histological features as described in the WHO classification. However, the translation to a final histological diagnosis is problematic; despite a good correlation between the individual histological features, we showed an inter-observer variation in the translation to a final diagnosis. Not only the recognition of the individual histological features is important, also their frequency, ranking and combination play a role in the pathologists decision to assign the bone marrow biopsy to ET, PV or PMF. These aspects are not clearly defined in the WHO classification and suggest that additional agreement on the definitions of the histological characteristics would be valuable.

In chapter 2, 4 and 5 we focus on the molecular mechanisms involved in the pathogenesis of MPN. The JAK2 ${ }^{\text {V617F }}$ mutation in MPN patients results in a sustained JAK2 proliferation leading to an increased activation of the signal transducer and activator of transcription (STAT) family. Especially STAT3 and STAT5 seems to play an important role in MPN development. STAT3 regulates cell growth and is involved in the megakaryopoiesis. STAT5 also regulates cell growth and inhibits the megakaryocyte apoptosis. The net result of STAT3 and STAT5 phosphorylation is proliferative activity and apoptosis inhibition of mainly megakaryocytes. In chapter 4 we stained bone marrow of MPN and control patients immunohistochemically with phosphorylated STAT3 (pSTAT3) and STAT5 (pSTAT5). pSTAT3 was more expressed in PMF and JAK2 ${ }^{\mathrm{V} 617 F}$ positive patients compared to the control and ET patients. pSTAT5 expression showed an increasing trend in PV and JAK2 V617F positive patients. We also demonstrate a correlation between microvessel density (MVD) and pSTAT5 expression.

The reduced life expectancy of PV and PMF patients can mainly be attributed to the increased vessel density and the increasing development of fibrosis in the bone marrow during the disease course. Galectin-1 (gal-1) is known to be involved in tumour angiogenesis and galectin-3 (gal-3) in liver fibrosis.

In chapter 4 we show that the gal-1 expression is significantly higher in the whole group of MPN patients compared to the control group. This finding, together with the finding of a significant correlation of the gal-1 expression with the MVD suggest a possible role of gal-1 in the increased angiogenesis seen in the bone marrow of MPN patients. Since it was demonstrated that angiogenesis is inhibited via gal-1 with the angiogenesis inhibitor 
anginex, it might even be a probable angiogenesis inhibitor in MPN patients in the future. The sustained JAK2 activation leads also to a sustained activation of the extracellular signal-regulated kinase (Erk) and the phosphatidylinositol-3-kinase (PI3K)/Akt/p70S6k/ mammalian target of rapamycine (mTor) signalling pathway. Activation of the Erk and PI3K/ Akt pathway results in apoptosis inhibition especially of megakaryocytes.

In chapter 5 we stained MPN and control bone marrow immunohistochemically with pErk, pAkt, p70S6k and Bnip3 (apoptosis activator) along with the MVD to characterize abnormal activation of these pathways and abnormal apoptotic responses in bone marrow and megakaryocytes of MPN patients. We show that increased bone marrow cellularity in MPN patients might be influenced by increased pErk, pAkt and decreased Bnip3 expression. A dominant role for megakaryocytes in especially ET patients was shown. Increased amounts of megakaryocytes in MPN patients can be due to increased pAkt and p70S6k.

The JAK2 $2^{V 617 F}$ mutation is present in over $95 \%$ of the PV patients and in approximately half of the ET and PMF patients. The discovery of the JAK2 ${ }^{\mathrm{V} 617 F}$ mutation has generated an interest in the development of JAK2 inhibitors. In chapter 6 we analysed the expression of gal-1, gal3, pSTAT3, pSTAT5, pErk and pAkt in JAK2 ${ }^{\mathrm{V} 617 F}$ mutation positive patients with myelofibrosis who were treated with the JAK1/2 inhibitor INCB018424, Ruxolitinib. Ruxolitinib is a JAK1/2 inhibitor which showed great improvements in clinical symptoms in MPN patients. We tried to correlate this improvement with a decrease in molecular markers in bone marrow. In this pilot study with only seven patients we demonstrated a decrease in pSTAT3, pSTAT5, pErk and pAkt expression in patients who were treated with Ruxolitinib during one year compared to patients who did not receive Ruxolitinib. However, other studies also have shown a suppression of these molecular markers in MPN patients without the JAK2 ${ }^{\text {V617F. This }}$ indicates that other pathways are involved in the JAK2 activation in MPN patients.

For gal-1 and gal-3 a lower expression was seen in patients treated with Ruxolitinib but also in patients who were not treated with Ruxolitinib. Therefore, we conclude that the expression of gal-1 and gal-3 is independent of JAK2 activation.

Unfortunately, in our pilot study no effect was seen on the grading of fibrosis after Ruxolitinib treatment, as fibrosis indicates a worse disease prognosis. If the underlying mechanism of fibrosis development in MPN patients can be revealed it would open up the possibilities for important breakthroughs in therapeutical treatment options. This would result in a better prognosis for MPN patients and probably longer survival rates especially in PMF, post-ET MF and post-PV MF patients. Although this study was done on only seven MPN patients with fibrosis, it underlines the importance of the discovered JAK2 ${ }^{\text {V617F }}$ mutation in the pathogenesis of MPN patients with fibrosis. 


\section{SAMENVATTING}

Myeloproliferatieve neoplasia (MPN) zijn aandoeningen die gelokaliseerd zijn in het centrale merg van de botten en een toename geven van verschillende onderdelen van het bloedvormende beenmerg. De ziekte die uitgaan van de rode reeks, simpel gezegd verantwoordelijk voor o.a. een grote toename van rode bloedcellen, noemen we polycythaemia vera (PV). Een proliferatie van de bloedplaatjes en de megakaryocyten, cellen die deze plaatjes vormen, zien we met name bij trombocythemie (ET). Proliferatie van fibroblasten, cellen van stroma in de tussenstof, noemen we een primaire myelofibrose (PMF). Het onderzoek in dit proefschrift is met name gericht op deze drie ziekten. De ziekte die een toename geeft van de witte reeks noemen we een myeloide leukemie, hetgeen berust op een ander ontstaansmechanisme en niet in dit proefschrift wordt besproken.

In de laatste jaren is duidelijk geworden dat het microscopisch beeld van de beenmergcellen een belangrijk onderdeel is van de MPN diagnose en onderverdeling van deze ziekten. Mede door de ontdekking van de JAK2 ${ }^{\text {V617F }}$ mutatie in het genetisch materiaal van het bloed, is meer inzicht verkregen in de pathogenese van MPN.

In hoofdstuk 1 en 2 beschrijven we de klinische en histologische criteria van MPN en de symptomen van MPN patiënten. De symptomen die MPN patiënten hebben, vertonen een grote overlap waardoor deze drie entiteiten klinisch moeilijk van elkaar te onderscheiden zijn. Beenmerg pathologie is mede een belangrijk onderzoek voor een definitieve diagnose. Beenmerg van patiënten met ET wordt voornamelijk gekenmerkt door een megakaryocyten proliferatie waarbij de megakaryocyten in losse clusters liggen. PV toont een trilineaire proliferatie in het beenmerg, maar de erythroïde cellijn is overwegend aanwezig. Beenmerg van PMF patiënten vertoont reticuline fibrose en proliferatie van de megakaryocyten waarbij de megakaryocyten dicht tegen elkaar in groepen geclusterd zijn. Sommige PV en ET patiënten vertonen ook reticuline fibrose in het beenmerg, bekend als post-ET myelofibrose (post-ET MF) en post-PV myelofibrose (post-PV MF) respectievelijk. De aanwezigheid van de JAK2 $2^{\text {V617F }}$ mutatie in zowel ET, PV als PMF patiënten maakt het stellen van de diagnose makkelijker, maar onderling zijn deze ziekten moeilijker van elkaar te onderscheiden. De JAK2 ${ }^{\mathrm{V} 617 \mathrm{~F}}$ mutatie is aanwezig in ongeveer 95\% van de PV patiënten en bij ongeveer $50 \%$ van de ET en PMF patiënten. De morfologische en klinische overlap en de aanwezigheid van de JAK2 $2^{\text {vil7F }}$ mutatie in MPN patiënten zaaien twijfel over het gegeven of ET, PV en PMF wel drie verschillende ziekten zijn danwel uitingen van één ziekte. Derhalve wordt ook voorgesteld om de MPNs in JAK2 positieve MPNs en JAK2 negatieve MPNs te verdelen met een onderverdeling in patiënten met en 
zonder myelofibrose. Omdat echter niet alle patiënten met een MPN een JAK2 mutatie hebben lijken ook nog andere, nog niet ontdekte oorzaken van belang bij het ontstaan van deze ziekte. Verder geven we een kort overzicht van de behandeling van MPN patiënten. De behandeling van de individuele MPNs is afhankelijk van de risico categorie waartoe de patiënten behoren. ET en PV patiënten die behoren tot de lage en intermediaire risico groep worden behandeld met een lage dosis aspirine en bij PV patiënten wordt daar phlebotomie (aderlating) aan toegevoegd. ET en PV patiënten die behoren tot de hoge risicogroep worden naast een lage dosis aspirine en eventueel phlebotomie bij PV patiënten ook behandeld met een chemotherapeuticum. Bij PMF patiënten die behoren tot de lage en intermediair-1 risico categorie is een afwachtende benadering gerechtvaardigd. De intermediair-2 en hoog risico groep krijgt chemotherapie of er zal overleg plaatsvinden of de patiënt in aanmerking komt voor een allogene stamcel transplantatie dan wel voor experimentele medicijnen.

In hoofdstuk 3 hebben we de reproduceerbaarheid bepaald van de huidige individuele morfologische kenmerken zoals beschreven in de WHO-classificatie en we hebben de reproduceerbaarheid van de histologische diagnose bepaald. De ontdekking van de JAK2 ${ }^{\text {V617F }}$ mutatie resulteerde in een herziening van de WHO-diagnostische criteria van de MPNs in 2008. Echter, bezorgdheid bestaat over de reproduceerbaarheid van de morfologische kenmerken zoals vermeld in de WHO 2008 criteria. Dit blijkt uit onze studie waarin we een hoge mate van consensus aantonen voor de afzonderlijke histologische kenmerken zoals beschreven in de WHO-classificatie. Echter, de vertaling naar een definitieve histologische diagnose is problematisch, ondanks een goede correlatie tussen de verschillende histologische kenmerken toonden we een inter-observer variatie aan in de vertaling naar een definitieve diagnose. Niet alleen de erkenning van de individuele histologische kenmerken is van belang, ook de frequentie, de rangschikking en de combinatie van de histologische kenmerken spelen een rol bij de beslissing van pathologen om een beenmerg biopsie te diagnosticeren als ET, PV of PMF. Echter, deze aspecten zijn niet duidelijk omschreven in de WHO-classificatie en suggereert dat aanvullende afspraken over de definities van de histologische kenmerken van belang kunnen zijn om tot de juiste diagnose te komen.

In hoofdstuk 2, 4 en 5 richten we ons op de moleculaire mechanismen die betrokken zijn bij de pathogenese van MPN. De JAK2 $2^{\text {V617F }}$ mutatie in MPN patiënten resulteert in een aanhoudende JAK2 proliferatie met als gevolg een verhoogde activering van de signaal transducer en activator van transcriptie (STAT) familie. Vooral STAT3 en STAT5 lijken een belangrijke rol te spelen in de MPN ontwikkeling. STAT3 is verantwoordelijk voor de 
celgroei en is betrokken bij de megakaryopoïese. STAT5 is ook verantwoordelijk voor de celgroei en verder remt STAT5 de megakaryocyte apoptose. Het netto resultaat van STAT3 en STAT5 fosforylering is proliferatieve activiteit en apoptose remming vooral van de megakaryocyten.

In hoofdstuk 4 hebben we beenmerg immunohistochemisch gekleurd van MPN patiënten en van controle patiënten met gefosforyleerd STAT3 (pSTAT3) en STAT5 (pSTAT5). pSTAT3 kwam meer tot expressie in PMF en JAK2 ${ }^{\text {V617F }}$ positieve patiënten in vergelijking met controle en ET patiënten. pSTAT5 expressie toonde een trend in PV en JAK2 ${ }^{\text {V617F }}$ positieve patiënten. We hebben ook een verband aangetoond tussen densiteit aan bloedvaten (MVD) en de pSTAT5 expressie. De verminderde levensverwachting van PV en PMF patiënten is vooral te wijten aan de verhoogde vaatdichtheid en de toenemende ontwikkeling van fibrose in het beenmerg tijdens het ziekteverloop. Galectine-1 (gal-1) is bekend betrokken te zijn in tumor angiogenese en galectine-3 (gal-3) in leverfibrose.

In hoofdstuk 4 laten we zien dat gal-1 significant meer tot expressie komt in de totale MPN groep vergeleken met de controlegroep. Deze bevinding, in combinatie met de vaststelling van een significante correlatie van de gal-1 expressie met de vaatdichtheid suggereren een mogelijke rol van gal-1 in de verhoogde angiogenese in het beenmerg van MPN patiënten. Eerdere studies laten zien dat angiogenese wordt geremd via gal-1 met de angiogeneseremmer anginex, daarom kan anginex zelfs een mogelijk angiogenese inhibitor in MPN patiënten in de toekomst zijn. De aanhoudende JAK2 activatie leidt ook tot een aanhoudende activatie van het extracellulair signaal gereguleerde kinase (Erk) en fosfatidylinositol-3-kinase (PI3K)/Akt/p70S6k/mammalian doel van rapamycine (mTOR) signaalweg. Activering van de Erk en PI3K/Akt signaalweg leidt tot een remming van de apoptose met name van de megakaryocyten.

In hoofdstuk 5 hebben we MPN beenmerg en controle beenmerg immunohistochemisch gekleurd met pErk, pAkt, p70S6k en Bnip3 lactiveert apoptose) samen met de vaatdichtheid om abnormale activatie van deze routes en abnormale apoptotische responsen in het beenmerg en in de megakaryocyten van MPN patiënten te achterhalen. We tonen aan dat de verhoogde cellulariteit in het beenmerg van MPN patiënten mogelijk wordt beïnvloed door de toegenomen pErk, pAkt en verminderde Bnip3 expressie. Een dominante rol voor de megakaryocyten, in het bijzonder ET patiënten, werd aangetoond. Verhoogde hoeveelheden megakaryocyten in MPN patiënten kunnen verklaard worden door toegenomen pAkt en p70S6k. 
De $J A K 2^{V 617 F}$ mutatie is aanwezig in meer dan $95 \%$ van de $P V$ patiënten en in ongeveer de helft van de ET en PMF patiënten. De ontdekking van de JAK2 $2^{\mathrm{V} 617 F}$ mutatie leidde tot de ontwikkeling van JAK2 remmers.

In hoofdstuk 6 hebben we de expressie van gal-1, gal-3, pSTAT3, pSTAT5, pErk en pAkt geanalyseerd in JAK2 ${ }^{\mathrm{V} 617 F}$ positieve patiënten met myelofibrose die werden behandeld met de JAK1/2 remmer INCB018424, Ruxolitinib. Ruxolitinib is een JAK1/2 remmer, die grote verbeteringen in de klinische symptomen van MPN patiënten vertoonden. We probeerden deze verbetering te correleren met een afname van moleculaire markers in het beenmerg. In de pilot-studie, slechts zes patiënten, toonden we een daling aan van de pSTAT3, pSTAT5, pErk en pAkt expressie bij patiënten die werden behandeld met Ruxolitinib gedurende een jaar in vergelijking met patiënten die niet werden behandeld met Ruxolitinib. Andere studies tonen ook een onderdrukking van deze moleculaire markers aan in MPN patiënten zonder de $J A K 2^{V 617 F}$ mutatie. Dit geeft aan dat andere signaal routes betrokken zijn bij de activatie van JAK2 in MPN patiënten.

Voor gal-1 en gal-3 werd een lagere expressie waargenomen bij patiënten die behandeld werden met ruxolitinib maar ook bij patiënten die niet werden behandeld met ruxolitinib. Daarom is de expressie van gal-1 en gal-3 onafhankelijk van de JAK2 activatie.

Er werd geen effect gezien op de mate van fibrose met Ruxolitinib behandeling, dat is teleurstellend, aangezien fibrose een slechtere prognose geeft van de ziekte. Als het onderliggende mechanisme van fibrose in MPN patiënten ontdekt wordt zal een belangrijke doorbraak in therapeutische opties mogelijk zijn, waardoor een betere prognose voor MPN patiënten ontstaat en waarschijnlijk een langere overleving met name voor de PMF, post-ET MF en post-PV MF patiënten. Hoewel deze studie werd gedaan bij slechts zes MPN patiënten met fibrose onderstreept het het belang van de ontdekte JAK2 ${ }^{\mathrm{V} 617 F}$ mutatie in de pathogenese van MPN patiënten met fibrose. 


\section{DANKWOORD}

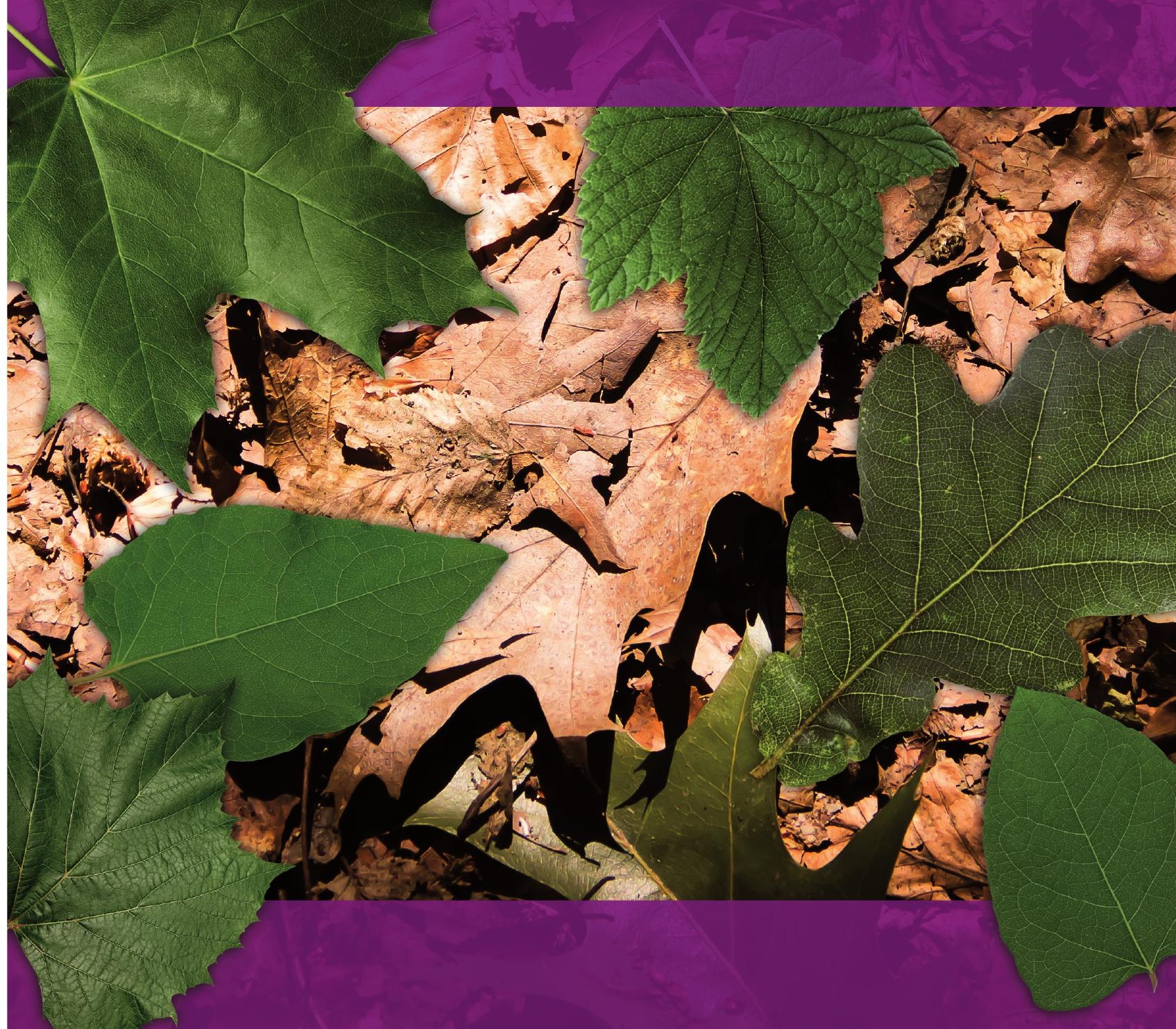


Als laatste een woord van dank. Vele mensen zijn bewust dan wel onbewust betrokken geweest bij het bereiken van deze mijlpaal en daar ben ik iedereen ontzettend dankbaar voor.

Allereerst Professor Dr. H.C. Schouten, één van mijn promotoren en haematoloog in het MUMC+.

Beste Harry, bij u (ik blijf u zeggen) ben ik als eerste terecht gekomen. Een onzekere studente die na het behalen van haar Biologische Gezondheidskunde diploma aangeraden werd om promotie onderzoek te gaan doen. Ik heb lang getwijfeld, de voor- en nadelen afgewogen en uiteraard lang nagedacht over het onderzoek in welk vakgebied. Voor mij was al heel snel duidelijk dat ik iets met oncologie/haematologie wilde doen. Op de één of andere manier heb ik toen de stoute/zenuwachtige schoenen aangetrokken en heb ik een afspraak met u gemaakt. Ik ervoer dat gesprek als zeer prettig, waarbij u mij de voor- en nadelen nog eens op een rijtje zette. Ongeveer een week later kreeg u een verzoek voor een promotie onderzoek naar de myeloproliferatieve afwijkingen. $U$ heeft geen moment geaarzeld en mij meteen gemaild. Tsja, toen was de knoop doorgehakt voor mij, ik ging promotie onderzoek combineren met de studie geneeskunde.

De eerste twee jaren had ik weinig contact met $u$, tot de laatste 2-3 jaar aanbraken. Door het toch wel wat dramatische beloop met m'n begeleiders die vertrokken, de onderzoeksgroep waar ik bij hoorde vertrok en m'n promotor die vertrok, wilde u iedere maand een afspraak maken om een vinger aan de pols te houden. Dit heb ik als zeer prettig ervaren en hier ben ik u heel erg dankbaar voor!

Inmiddels is het voor mij duidelijk geworden dat ik haematologe wil worden waarbij u mijn grote voorbeeld bent zowel de manier waarop u met patiënten omgaat als de manier waarop u met studenten en AIOS omgaat. Ik zal u zeker nog tegenkomen gedurende mijn AIOS plek in Maastricht en wie weet heb ik de grote eer om nog eens als haematologe heel klein naast u te mogen staan.

Professor Dr. A. zur Hausen, één van mijn promotoren en patholoog in het MUMC+. Beste Axel, voor ons beiden was het een lastige situatie. Nadat m'n promotor van de pathologie afdeling was vertrokken heb ik een tijdje geen promotor gehad, totdat $u$ het nieuwe hoofd van de afdeling pathologie werd. In overleg met elkaar werd u mijn promotor. Ik wil u dan ook hartelijk bedanken voor de kritische noot bij mijn laatste artikelen.

Dr. A.M.W. van Marion, copromotor en patholoog in het VieCuri te Venlo.

Beste Ariënne, vanaf het begin van mijn promotie was je via Freek zijdelings betrokken bij mijn onderzoek, maar om nou te zeggen dat we elkaar goed kende... nee. Het omslagpunt 
kwam toen Freek wegging en jij heel dapper de begeleiding van mij op je wilde nemen. Ik was en ben je er nog steeds ontzettend dankbaar voor. Tot mijn grote verdriet kreeg ik bijna een jaar na het vertrek van Freek op een vrijdag tegen het eind van de middag tijdens mijn GEZP stage op de haematologie afdeling in het azM een mailtje van je; het was een lang verhaal, maar de boodschap was duidelijk....je ging weg naar Venlo. Ik heb die middag een potje zitten huilen en gedacht ik stop ermee, de tweede begeleider die nu weggaat en wie kan het overnemen? Met die gedachte heb ik een tijdje rondgelopen, maar jij wilde koste wat kost mijn begeleiding blijven doen omdat er niemand anders was om het over te nemen en je niet wilde dat ik hiervan de dupe zou worden. Ik zal je hiervoor altijd dankbaar blijven! Ik heb ontzettend veel van je geleerd als we weer eens met $z$ 'n tweeën door de microscoop aan het turen waren, we aan het brainstormen waren over hoe de kleuringen te verbeteren en je was altijd positief als ik het even niet zag zitten. Ook de kletsmomenten over de gewone dingen van het leven vond ik altijd erg gezellig en aangenaam.

Ariënne, ik wil je ontzettend bedanken voor je grote inzet en steun, zonder jouw begeleiding was ik zeker gestopt, je opkomen voor “jouw” promovenda als dat nodig was, de gezellige kletsmomenten en de vele vele uurtjes die je erin hebt gestoken naast je gewone werk waar ik veel bewondering voor heb.

Dr. F.J. Bot, patholoog in het Haga ziekenhuis te Den Haag.

Beste Freek, toen Harryeen aanbieding kreeg voor een onderzoek naar myeloproliferatieve afwijkingen was jij als haemato-patholoog de aangewezen persoon om mijn begeleiding te gaan doen. Al snel kwamen er een aantal ideeën op tafel waar we vol goede moed mee aan de slag gingen. Het ging uiteraard niet zo snel in verband met mijn studie, maar ondertussen heb ik gemerkt dat het ook voor jullie best wel prettig is om niet de hele tijd een zeurende promovendus aan de deur te hebben staan met lastige vragen als "heb je al tijd gehad om naar het Excel bestand te kijken?" "heb je vandaag tijd om coupes te kijken?" en de meest lastige vraag "heb je het artikel al nagekeken?". Helaas kwam aan onze samenwerking een einde toen je besloot naar Den Haag te vertrekken, ik vond het uiteraard heel erg jammer en achteraf bleek dat je niet de eerste zou zijn die vertrok naar een ander ziekenhuis. We spraken af contact te houden en Ariënne en ik hebben je gevraagd om mee te blijven denken met mijn promotie en of je artikelen wilde mee beoordelen. Helaas, roet werd in het eten gegooid toen je ziek werd, ik schrok erg toen je me belde een paar dagen voor de kerst van 2009. Gelukkig gaat alles nu weer goed met je. Beste Freek, het gaat goed met je en dat doet me goed. Je hartelijkheid als ik binnen wandelde was altijd erg aangenaam, ik voelde me zeer welkom bij je. Ik wil je ontzettend bedanken voor je kennis die je hebt overgebracht over de MPNs, de tijd die je erin 
hebt gestoken om samen te kijken naar de beenmergcoupes van de patiënten en me langzaamaan hebt geleerd wat de kenmerken zijn van de myeloproliferatieve afwijkingen. Ook de vele gezellige en vaak ook onzinnige kletsmomenten zal ik niet vergeten.

Dr. K.H. Lam en Dr. K.M. Hebeda, patholoog in het Erasmus M.C te Rotterdam en pathologe in het Radboud ziekenhuis te Nijmegen respectievelijk.

Beste King en Konnie, ik leerde jullie kennen via Freek en Ariënne toen jij, Konnie, het idee opvatte om de WHO criteria eens onder de loep te nemen. Vaker hebben we uitstapjes gemaakt naar Rotterdam en Nijmegen en dat was altijd leerzaam en leuk. Ik herinner me m'n allereerste presentatie in Bordeaux nog. Ik presenteerde onze data en jeetje...wat was ik zenuwachtig. Ik zie jullie nog zitten op het puntje van jullie stoel, ook gespannen, maar zodra ik de eerste zin had gesproken leunde jullie achterover en zeiden later tegen me..."toen je eenmaal de eerste zin had uitgesproken, konden we rustig achterover leunen, zelfs de missende slide heb je goed opgepakt!". Ook van jullie heb ik veel geleerd op de momenten dat we met elkaar om de tafel zaten. King jij hebt regelmatig met me gesproken over de opleiding tot internist en hoe te combineren met promotie en dat ik af en toe toch echt meer met m'n vuist op tafel moest slaan. Hahaha...dat laatste is en blijft een moeilijk punt voor me, maar ik hou het altijd in m'n achterhoofd. King en Konnie, heel erg bedankt!

Lieve Kimsie (Kim), ik was heel erg blij toen jij ook je promotie onderzoek startte in het MUMC+. We hebben vele koffiemomenten gehad en elkaar op bepaalde gebieden zeker kunnen helpen al zit jij in een ander vakgebied. Buiten het werk om ben je een van m'n beste vriendinnen. Ik heb zo'n ontzettende bewondering voor jou; hoe je alles hebt aangepakt tijdens je studie, de moeilijke dingen waar je toen al tegen aan liep en vervolgens $A(N) I O S$ combineren met je gezinsleven. Je bent en blijft altijd positief, je hebt een luisterend oor en je hebt me ontzettend gesteund in een van m'n moeilijkste periodes. Onze vriendschap lijkt net vanzelf te gaan en dat is héél erg prettig en ben dan ook blij dat je mijn paranimf wilt zijn!

Lieve Kim, ik wil je heel erg bedanken voor de gezellige momenten en de tijd dat je me gesteund hebt toen ik het zo nodig had. Je zei de waarheid tegen me en dat is uiteraard niet altijd leuk, maar heel goed geweest. Meis, ik wens je heel veel succes nu je bij de gynaecologie in Heerlen gestart bent. Je wordt een fantastische gynaecologe, daar ben ik van overtuigd en we blijven de gezellige momenten erin houden! 
Lieve Flo, Floor, jij en ik lijken in veel opzichten op elkaar. We deelden dezelfde kamer, nog net niet dezelfde stoel, en we hadden nog iets gemeen tijdens ons promotie onderzoek; we waren een vreemde eend in de bijt. Dit is voor ons beiden niet altijd even makkelijk geweest, maar gelukkig konden we bij elkaar spuien! Nu zit je in Amsterdam en heb je eindelijk een heerlijk eigen stekkie samen met Bas, tenminste als jullie niet weer eens apart voor jullie werk de wereld over vliegen. Dit heeft je veel rust opgeleverd. Meis, twijfel vooral niet aan jezelf, je bent een ontzettende slimme meid en ik ben er van overtuigd dat je er zeker gaat komen welke kant je ook uitgaat. Blijf vooral bij jezelf en kom voor jezelf op, want niemand anders doet dat voor je! Ik hoop dat we de gezellige etentjes en bioscoop bezoekjes kunnen uitbreiden met weekendjes in Amsterdam en Maastricht.

Lieve Anne, we kennen elkaar al wat langer van Gezondheidswetenschappen, maar zijn pas echt goede vriendinnen geworden sinds de studie geneeskunde. Lieve meis, je hebt het niet makkelijk gehad en heb een grote bewondering voor je doorzettingsvermogen! Je bent in opleiding tot kinderarts en elk kind dat jou tegenkomt in het ziekenhuis als arts mag in zijn/haar handjes wrijven met jou! Ook jij bent een steun voor me geweest de laatste tijd en bij jou kon ik uit huilen en hahaha... dan zat jij ook met tranen in je ogen! Meiske, we zien elkaar niet veel, maar weet dat ik altijd voor je klaar sta. Je wordt een fantastische kinderarts, maar denk ook aan jezelf en geef je grenzen aan! De gezellige momenten met Kim houden we erin!

Uiteraard zijn er meer mensen die ik graag wil bedanken voor de afgelopen jaren. Allereerst de analisten: Petra, Peter, Edith, Kim W, Andrea, Sarah en Guido voor jullie kennis en het aanleren van de immuno's en de gezellige klets momenten op het lab.

Benoit dank je wel voor het overnemen van de machinale kleuringen dat heeft me veel tijd gescheeld!

Jack dank je wel voor quantimet-programma's wat moesten we toch zonder jou!

Ook dank je wel aan de "kleine demo" kamergenootjes, Karin, Karen, Joep, Tjinta, Anette, Thomas, David en Timo voor de hilarische momenten en gezellige sfeer!

Lieve Eef, Leintje en Lian, vriendinnen vanaf de middelbare school en voor het leven. We zien elkaar niet veel en spreken elkaar niet veel, maar als we elkaar zien dan is het altijd bere gezellie en we kunnen altijd op elkaar rekenen. Als er iets is dan staan we voor elkaar klaar en dat is echte vriendschap. Van jullie heb ik heel veel steun gehad de laatste tijd wat ik erg hard kon gebruiken. Lieve meiden, ik ben ontzettend blij met jullie als vriendinnen en dat reisje naar Rome moeten we nu toch echt een keer gaan plannen!!!! 
Tineke, Ilona, Marielle en Kaythlin, ook super lieve en goede vriendinnen, met jullie heb ik ook veel meegemaakt. Hele gezellige momenten, lekker uit ons dak gaan met stappen, maar ook de serieuze dingen van het leven. Ook jullie wil ik heel erg bedanken voor alle steun. ledereen heeft nu wel zo'n beetje haar stekkie gevonden, alles valt op zijn plaats. Kortom, het komt wel goed met jullie!

Iris, Veerle, Hanneke, Ingrid, Joep, Jürgen en andere oud-epi's die ik eventueel vergeten ben, dank jullie wel voor de gezellige kletsmomenten en dat ik me bij jullie groep mocht aansluiten.

Cor en Elly en de dames van het secretariaat, jullie ook heel erg bedankt dat ik altijd mocht binnenvallen voor de administratieve en financiële hulp en de leuke en serieuze gesprekken die daaruit voortvloeiden.

Ook Matthijs wil ik bedanken voor het oplossen van alle computerproblemen, zonder hem had ik m'n laptop al lang uit het raam gegooid!

Verder wil ik iedereen die ik nog vergeten ben en iedereen van de pathologie afdeling bedanken voor de interesse, hulp en gezellige kletsmomenten.

Verder wil ik mijn collega-AIOS en bazen van de afdeling interne geneeskunde in het MUMC+ bedanken voor de interesse in mijn promotie en de leuke samenwerking. Ook mijn meest recente nieuwe collega-AIOS en bazen in het Atrium te Heerlen wil ik bedanken voor de interesse en de gezelligheid en de snelheid waarmee jullie mij een welkom gevoel gaven.

Lieve Jasper, we kennen elkaar nog niet zo lang, maar hebben al wel wat hobbels moeten nemen. Vanaf het begin heb je me gesteund en het me op meerdere fronten makkelijk gemaakt en ik heb het jou juist héél moeilijk gemaakt. Ik ben je dankbaar voor je geduld en liefde! En het lijkt erop dat we eindelijk een beetje onze weg met z'n tweeën hebben gevonden. 
Last but definitely not least...

Lieve pap \& mam en Robert-Jan, aan jullie ben ik mijn grootste dank verschuldigd! Jullie zijn mijn steun en toeverlaat geweest en nog steeds. Op jullie heb ik altijd kunnen rekenen en mijn verhalen, boosheid, frustraties en verdriet altijd kunnen vertellen. Robert-Jan jij weet als geen ander hoe het is om te promoveren en we konden bij elkaar de frustraties goed kwijt. Ik ben ontzettend trots op je wat je allemaal bereikt hebt. Je hebt alles achter je gelaten in Nederland en hebt weer een nieuw leven opgebouwd in Engeland en nu sinds kort ben je aan een nieuw leven begonnen in Oostenrijk. Hierdoor zien we elkaar heel erg weinig en zien en spreken we elkaar voornamelijk via de skype en dat is niet altijd even leuk. Maar ik geniet wel van de gesprekken via skype en heb ook heel veel steun gehad aan je wijze woorden en manier van naar bepaalde dingen in het leven te kijken en daar ben ik je heel dankbaar voor! Ik kan me geen betere en vooral ook geen lievere broer wensen. In Engeland heb je ook Beckie leren kennen en in elkaar hebben jullie gevonden wat jullie zochten. Ik ben ontzettend blij voor jullie en wens jullie alle geluk toe en hopelijk over een paar jaar een eigen stekkie met z'n tweeën op het vaste land van Europa .

Pap en mam, ik ben jullie heel dankbaar voor de steun, stimulatie, de mogelijkheden en vooral de liefde die jullie ons altijd hebben gegeven. Het doorzettingsvermogen, het verantwoordelijkheidsgevoel hebben we echt van jullie geërfd wat ook zo zijn nadelen kan hebben.

Hoe jullie het hebben volgehouden de laatste tijd met mij weet ik ook niet, maar ik besef me maar al te goed dat jullie het niet makkelijk met me hebben gehad afgelopen jaar. Een zware periode, op meerdere uiteenlopende fronten, kenmerkte helaas het einde van m'n promotie, maar jullie zijn er altijd voor me geweest en ik kon altijd bellen dag en nacht. De momenten dat ik thuis ben of wanneer jullie in Maastricht zijn vind ik heerlijk! Leuke en gezellige dingen, maar mam we moeten echt meer dagjes shoppen hoor... en ach dan nemen we pap wel weer mee, vindt hij stiekem veel te leuk. Ik ben jullie ontzettend dankbaar en kan me geen betere ouders wensen. Lieve pap \& mam en R-J\&Beckie, ik hou ontzettend veel van jullie en ben jullie ongelooflijk dankbaar voor alles. 



\section{ABOUT THE AUTHOR}

\section{LIST OF PUBLICATIONS}

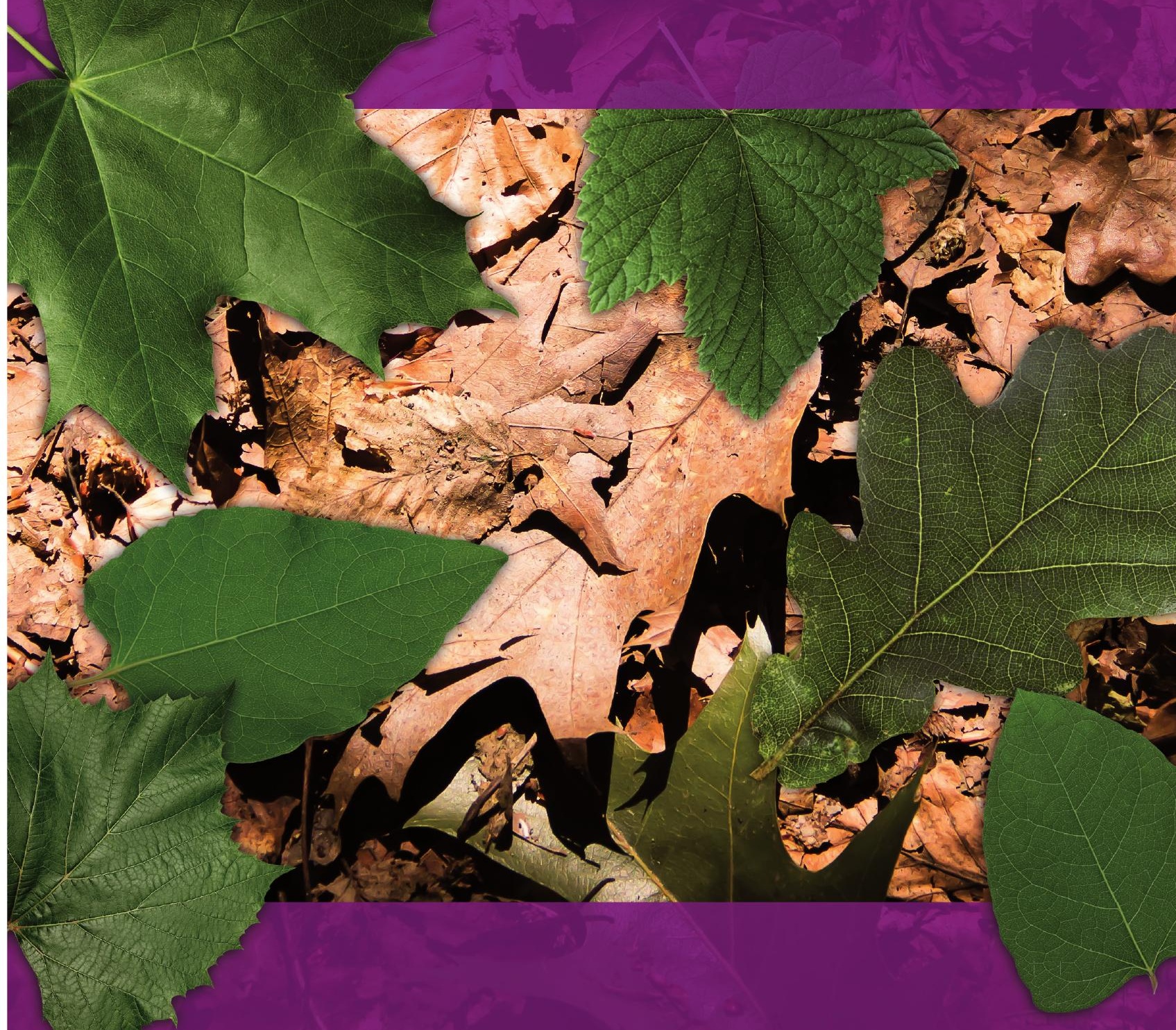




\section{ABOUT THE AUTHOR}

Ik ben geboren op 13 januari 1983 in Tiel. Na 6 jaar in Tiel te hebben gewoond ben ik samen met mijn ouders en broer verhuisd naar Overdinkel. In Overdinkel zat ik op de basisschool "De Pax Christie" en in 1994 ben ik naar "Het Jacobus College" in Enschede gegaan waar ik in 2001 mijn Gymnasium diploma haalde. In 2001 ben ik begonnen met de studie Biologische Gezondheidskunde (BGK) aan de Universiteit van Maastricht met een eindstage bij Gezondheidsrisico Analyse en Toxicologie en behaalde in 2005 mijn diploma. Tijdens mijn studie Biologische Gezondheidskunde ben ik in 2003 begonnen met het studeren van Geneeskunde aan de Universiteit van Maastricht na het wegwerken van mijn natuurkunde deficiëntie. Tijdens mijn studie geneeskunde ben ik studie met onderzoek gaan combineren in de vorm van Stud-AIO op de afdeling pathologie. In 2007 ben ik op de afdeling pathologie begonnen als Stud-AIO en in december 2009 ben ik fulltime verder gegaan met mijn promotie onderzoek met als titel "Myeloproliferative Neoplasia: more insight in the pathogenesis". Sinds december 2011 ben ik werkzaam als AIOS bij de interne geneeskunde van het Maastricht Universitair Medisch Centrum te Maastricht en per 1 februari 2012 werk ik als AIOS in het Atrium Medisch Centrum te Heerlen.

Suzanne Koopmans was born on the 13th of January 1983 in Tiel, the Netherlands. She grew up in Tiel and Overdinkel where she attended elementary school at "Pax Christie". From 1994 she attended higher secondary school at the "Jacobus College" in Enschede and obtained her diploma (Gymnasium) in 2001. In 2001 she started the study Health Sciences, Biological Health Sciences, at Maastricht University and graduated as MSc in 2005. During her health sciences study she started with Medical School at Maastricht University in 2003 and graduated as MD in December 2009. During her two studies she generated interest in science combined with a great interest in oncology. In 2007 she started her PhD program at the Department of Pathology at Maastricht University Medical Centre. During her PhD program, which she combined with the last 2 years of Medical School, she worked on a project entitled Myeloproliferative Neoplasia: more insight in the pathogenesis", which resulted in her dissertation.

In December 2011 Suzanne started her residency training Internal Medicine at the Maastricht University Medical Centre, Maastricht and is now working as resident at the Atrium Medical Centre, Heerlen. 



\section{LIST OF PUBLICATIONS}

1. Suzanne M. Koopmans, Freek J. Bot, King H. Lam, Ariënne M.W. van Marion, Hendrik de Raeve, Konnie M. Hebeda. Reproducibility of histological classification in non-fibrotic myeloproliferative neoplasia.

Am J Clin Pathol 2011. oct;136(4):618-624.

2. Koopmans SM, Bot F.J, Schouten H.C, van Marion A.M.W. The involvement of galectins in the modulation of the JAK/STAT pathway in myeloproliferative neoplasia. Am J Blood Res 2012. may 2(2):119-127.

3. Koopmans SM, van Marion A.M.W, Schouten H.C. Myeloproliferative neoplasia: a review of clinical criteria and treatment.

Neth J Med 2012. may;70(4):159-167.

4. Koopmans SM, Schouten H.C, van Marion A.M.W. The anti-apoptotic pathways in bone marrow and megakaryocytes in myeloproliferative neoplasia.

Pathobiology 2013. Nov;81(2):60-68.

5. Koopmans SM, Schouten H.C. Treatment options for myelofibrosis and myeloproliferative neoplasia.

International Journal of Hematologic Oncology 2013. Dec;2(6):487-495.

6. Suzanne M. Koopmans, Schouten H.C., van Marion A.M.W. BCR-ABL negative Myeloproliferative Neoplasia: a review of involved molecular mechanisms. Accepted in Histology and Histopathology 2014. 
